TOPICAL REVIEW

\title{
Quasinormal modes of black holes and black branes
}

\author{
Emanuele Berti ${ }^{1,2}$, Vitor Cardoso ${ }^{1,3}$, Andrei O. Starinets ${ }^{4}$ \\ ${ }^{1}$ Department of Physics and Astronomy, The University of Mississippi, \\ University, MS 38677-1848, USA \\ 2 Theoretical Astrophysics 130-33, California Institute of Technology, Pasadena, \\ CA 91125, USA \\ 3 Centro Multidisciplinar de Astrofísica - CENTRA, Departamento de Física, \\ Instituto Superior Técnico, Av. Rovisco Pais 1, 1049-001 Lisboa, Portugal \\ ${ }^{4}$ Rudolf Peierls Centre for Theoretical Physics, Department of Physics, \\ University of Oxford, 1 Keble Road, Oxford, OX1 3NP, United Kingdom \\ E-mail: berti@phy.olemiss.edu, vitor.cardoso@ist.utl.pt, \\ andrei.starinets@physics.ox.ac.uk
}

\begin{abstract}
Quasinormal modes are eigenmodes of dissipative systems. Perturbations of classical gravitational backgrounds involving black holes or branes naturally lead to quasinormal modes. The analysis and classification of the quasinormal spectra requires solving non-Hermitian eigenvalue problems for the associated linear differential equations. Within the recently developed gauge-gravity duality, these modes serve as an important tool for determining the near-equilibrium properties of strongly coupled quantum field theories, in particular their transport coefficients, such as viscosity, conductivity and diffusion constants. In astrophysics, the detection of quasinormal modes in gravitational wave experiments would allow precise measurements of the mass and spin of black holes as well as new tests of general relativity. This review is meant as an introduction to the subject, with a focus on the recent developments in the field.
\end{abstract}

PACS numbers: 04.70.-s, 04.30.Tv, 11.25.Tq, 11.10.Wx, 04.50.-h, 04.25.dg

\section{Contents}

1 Introduction 3

1.1 Milestones ....................... . . . . 7

1.2 Notation and conventions . . . . . . . . . . . . . 10

2 A black hole perturbation theory primer $\quad 11$

2.1 Perturbations of the Schwarzschild-anti-de Sitter geometry . . . . . . . 11

2.2 Higher-dimensional gravitational perturbations . . . . . . . . . . 15

2.3 Weak fields in the Kerr background: the Teukolsky equation . . . . . . 15

3 Defining quasinormal modes $\mathbf{1 7}$

3.1 Quasinormal modes as an eigenvalue problem . . . . . . . . 17

3.2 Quasinormal modes as poles in the Green's function . . . . . . . . . 18 
4 Computing quasinormal modes 20

4.1 Exact solutions . . . . . . . . . . . . . . . . . . 21

4.2 The WKB approximation . . . . . . . . . . . . 25

4.3 Monodromy technique for highly-damped modes . . . . . . . . . . 27

4.4 Asymptotically anti-de Sitter black holes: a series solution . . . . . . . 28

4.5 Asymptotically anti-de Sitter black holes: the resonance method . . . 30

4.6 The continued fraction method . . . . . . . . . . . . . . 30

5 The spectrum of asymptotically flat black holes 31

5.1 Schwarzschild . . . . . . . . . . . . . . . . . . . . . 31

5.2 Reissner-Nordström . . . . . . . . . . . . . . . . . . . 35

5.3 Kerr . . . . . . . . . . . . . . . . . . . . . . . 38

5.4 Kerr-Newman . . . . . . . . . . . . . . . . . . . . 41

5.5 Higher dimensional Schwarzschild-Tangherlini black holes . . . . . . . 41

6 The spectrum of asymptotically anti-de Sitter black holes 43

6.1 Schwarzschild anti-de Sitter black holes . . . . . . . . . . . . . . . 43

6.2 Reissner-Nordström and Kerr anti-de Sitter black holes . . . . . . . . 48

6.3 Toroidal, cylindrical and plane-symmetric anti-de Sitter black holes . . 49

7 The spectrum of asymptotically de Sitter and other black holes 49

7.1 Asymptotically de Sitter black holes . . . . . . . . . . . . . . . . 49

7.2 Black holes in higher-derivative gravity . . . . . . . . . . . . 50

7.3 Braneworlds . . . . . . . . . . . . . . . . . . . . 51

7.4 Black holes interacting with matter . . . . . . . . . . . . 51

8 Quasinormal modes and the gauge-gravity duality 51

8.1 The duality . . . . . . . . . . . . . . . . 51

8.2 Dual quasinormal frequencies as poles of the retarded correlators . . . 53

8.3 The hydrodynamic limit . . . . . . . . . . . . . . . 55

8.4 Universality of the shear mode and other developments . . . . . . . 58

9 Quasinormal modes of astrophysical black holes 59

9.1 Physical parameters affecting ringdown detectability . . . . . . . . 60

9.2 Excitation of black hole ringdown in astrophysical settings . . . . . . . 62

9.3 Astrophysical black holes: mass and spin estimates . . . . . . . . . . . 68

9.4 Detection range for Earth-based and space-based detectors . . . . . . 75

9.5 Event rates . . . . . . . . . . . . . . . . . . . . 77

9.6 Inferring black hole mass and spin from ringdown measurements . . . 79

9.7 Tests of the no-hair theorem . . . . . . . . . . . . . 82

9.8 Matching inspiral and ringdown: problems and applications . . . . . 84

10 Other recent developments 85

10.1 Black hole area quantization: in search of a $\log \ldots \ldots \ldots . . . . .85$

10.2 Thermodynamics and phase transitions in black hole systems . . . . . 86

10.3 Non-linear quasinormal modes . . . . . . . . . . . . . . 87

10.4 Quasinormal modes and analogue black holes . . . . . . . . . . 87

11 Outlook 89 


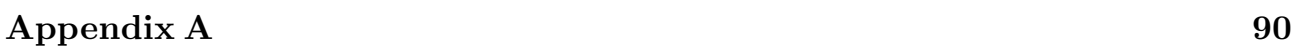

Appendix B 94

Appendix C

$\begin{array}{ll}\text { Appendix D } & 96\end{array}$

\section{Introduction}

"The mathematical perfectness of the black holes of Nature is [...] revealed at every level by some strangeness in the proportion in conformity of the parts to one another and to the whole." S. Chandrasekhar, "The Mathematical Theory of Black Holes"

Characteristic modes of vibration are persistent in everything around us. They make up the familiar sound of various musical instruments but they are also an important research topic in such diverse areas as seismology, asteroseismology, molecular structure and spectroscopy, atmospheric science and civil engineering. All of these disciplines are concerned with the structure and composition of the vibrating object, and with how this information is encoded in its characteristic vibration modes: to use a famous phrase, the goal of studying characteristic modes is to "hear the shape of a drum" 1. This is a review on the characteristic oscillations of black holes (BHs) and black branes (BHs with plane-symmetric horizon), called quasinormal modes (QNMs). We will survey the theory behind them, the information they carry about the properties of these fascinating objects, and their connections with other branches of physics.

Unlike most idealized macroscopic physical systems, perturbed BH spacetimes are intrinsically dissipative due to the presence of an event horizon. This precludes a standard normal-mode analysis because the system is not time-symmetric and the associated boundary value problem is non-Hermitian. In general, QNMs have complex frequencies, the imaginary part being associated with the decay timescale of the perturbation. The corresponding eigenfunctions are usually not normalizable, and, in general, they do not form a complete set (see Refs. 2, 3] for more extensive discussions). Almost any real-world physical system is dissipative, so one might reasonably expect QNMs to be ubiquitous in physics. QNMs are indeed useful in the treatment of many dissipative systems, e.g. in the context of atmospheric science and leaky resonant cavities.

Two excellent reviews on BH QNMs [4, 5] were written in 1999. However, much has happened in the last decade that is not covered by these reviews. The recent developments have brought $\mathrm{BH}$ oscillations under the spotlight again. We refer, in particular, to the role of QNMs in gravitational wave astronomy and their applications in the gauge-gravity duality. This work will focus on a critical review of the new developments, providing our own perspective on the most important and active lines of research in the field.

After a general introduction to QNMs in the framework of $\mathrm{BH}$ perturbation theory, we will describe methods to obtain QNMs numerically, as well as some important analytic solutions for special spacetimes. Then we will review the QNM spectrum of BHs in asymptotically flat spacetimes, asymptotically (anti-)de Sitter (henceforth AdS or dS) spacetimes and other spacetimes of interest. After this general overview we will discuss what we regard as the most active areas in QNM research. 
Schematically, we will group recent developments in QNM research into three main branches:

(i) AdS/CFT and holography. In 1997-98, a powerful new technique known as the AdS/CFT correspondence or, more generally, the gauge-string duality was discovered and rapidly developed [6]. The new method (often referred to as holographic correspondence) provides an effective description of a non-perturbative, strongly coupled regime of certain gauge theories in terms of higher-dimensional classical gravity. In particular, equilibrium and non-equilibrium properties of strongly coupled thermal gauge theories are related to the physics of higher-dimensional BHs and black branes and their fluctuations. Quasinormal spectra of the dual gravitational backgrounds give the location (in momentum space) of the poles of the retarded correlators in the gauge theory, supplying important information about the theory's quasiparticle spectra and transport (kinetic) coefficients. Studies of QNMs in the holographic context became a standard tool in considering the near-equilibrium behavior of gauge theory plasmas with a dual gravity description. Among other things, they revealed the existence of a universality of the particular gravitational frequency of generic black branes (related on the gauge theory side to the universality of the viscosity-entropy ratio in the regime of infinitely strong coupling), as well as intriguing connections between the dynamics of $\mathrm{BH}$ horizons and hydrodynamics 7 . The duality also offers a new perspective on notoriously difficult problems, such as the $\mathrm{BH}$ information loss paradox, the nature of $\mathrm{BH}$ singularities and quantum gravity. Holographic approaches to these problems often involve QNMs. This active area of research is reviewed in Section 8 ,

(ii) QNMs of astrophysical black holes and gravitational wave astronomy. The beginning of LIGO's first science run (S1) in 2002 and the achievement of design sensitivity in 2005 marked the beginning of an era in science where BHs and other compact objects should play a prominent observational role. While electromagnetic observations are already providing us with strong evidence of the astrophysical reality of BHs 8], gravitational wave observations will incontrovertibly show if these compact objects are indeed rotating (Kerr) BHs, as predicted by Einstein's theory of gravity. BH QNMs can be used to infer their mass and angular momentum [9] and to test the no-hair theorem of general relativity [10, 11. Dedicated ringdown searches in interferometric gravitational wave detector data are ongoing [12, 13]. The progress on the experimental side was accompanied by a breakthrough in the numerical simulation of gravitational wave sources. Long-term stable numerical evolutions of $\mathrm{BH}$ binaries have been achieved after 4 decades of efforts [14, 15, 16, confirming that ringdown plays an important role in the dynamics of the merged system. These developments are reviewed in Section 9

(iii) Other developments. In 1998, Hod suggested that highly-damped QNMs could bridge the gap between classical and quantum gravity [17. The following years witnessed a rush to compute and understand this family of highly damped modes. The interest in this subject has by now faded substantially but, at the very least, Hod's proposal has contributed to a deeper analytical and numerical understanding of QNM frequencies in many different spacetimes, and it has highlighted certain general properties characterizing some classes of $\mathrm{BH}$ solutions. These ideas and other recent developments (including a proposed connection between QNMs and $\mathrm{BH}$ phase transitions, the QNMs of analogue BHs, the stability of naked singularities and its relation with the so-called algebraically special modes) are reviewed in Section 10.

The present work is mostly intended to make the reader familiar with the new 
developments by summarizing the vast (and sometimes confusing) bibliography on the subject. We tried to keep the review as self-contained as possible, while avoiding to duplicate (as far as possible to preserve logical consistency) material that is treated more extensively in other reviews on the topic, such as Refs. 4, 5, 2, 18. A detailed understanding of BH QNMs and their applications requires some specialized technical background. QNM research has recently expanded to encompass a very wide range of topics: a partial list includes analogue gravity, alternative theories of gravity, higherdimensional spacetimes, applications to numerical relativity simulations, explorations of the gauge-gravity duality, the stability analysis of naked singularities and ringdown searches in LIGO. Because of space limitations we cannot discuss all of this material in detail, and we refer the reader to other reviews. Topics that are treated in more detail elsewhere include: (1) a general overview of gravitational radiation [19, 20] and its multipolar decomposition [21; (2) BH perturbation theory [22, 23, 24, 25, 26, 27]; (3) the issue of quantifying QNM excitation in different physical scenarios (see e.g. [28] for an introduction pre-dating the numerical relativity breakthroughs of 2005, and 29 for a more updated overview of the field); (4) tests of general relativity and of the no-hair theorem that either do not make use of ringdown 30 , or do not resort to gravitational wave observations at all [31, 32, 33; ; 5) BH solutions in higher dimensions [34; (6) many aspects of the gauge-gravity duality $7,35,36,37,38$. The reviews listed above provide more in-depth looks at different aspects of QNM research, but we tried to provide concise introductions to all of these topics while (hopefully) keeping the presentation clear and accessible.

Chandrasekhar's fascination with the mathematics of BHs was due to their simplicity. BHs in four-dimensional, asymptotically flat spacetime must belong to the Kerr-Newman family, which is fully specified by only three parameters: mass, charge and angular momentum (see e.g. Ref. [39, or Carter's contribution to Ref. [40]). One expresses this by saying that BHs have no hair (or more precisely, that they have three hairs). A consequence of the no-hair theorem is that all perturbations in the vicinities of a $\mathrm{BH}$ must decay to one and the same final state, i.e. that all hairs (except three) must be lost. Perturbative and numerical calculations show that the hair loss proceeds, dynamically, via quasinormal ringing. The gravitational wave signal from a perturbed $\mathrm{BH}$ can in general be divided in three parts: (i) A prompt response at early times, that depends strongly on the initial conditions and is the counterpart to light-cone propagation; (ii) An exponentially decaying "ringdown" phase at intermediate times, where QNMs dominate the signal, which depends entirely on the final BH's parameters; (iii) A late-time tail, usually a power-law falloff of the field [41. Mathematically, each of these stages arises from different contributions to the relevant Green's function (see Section 3.2). QNM frequencies depend only on the BH's parameters, while their amplitudes depend on the source exciting the oscillations.

Numerical and analytical analysis of processes involving BHs confirm these expectations. QNMs were observed for the first time in numerical simulations of the scattering of Gaussian wavepackets by Schwarzschild BHs in 1970, soon after the BH concept itself was introduced and popularized by John Wheeler. Vishveshwara 42 noticed that the waveform at late times consists of a damped sinusoid, with ringing frequency almost independent of the Gaussian's parameters. Ringdown was observed again in the linearized approximation to the problem of a test mass falling from infinity into a Schwarzschild $\mathrm{BH}$ [43. By now, decades of experience have shown that any event involving $\mathrm{BH}$ dynamics is likely to end in this same characteristic way: the gravitational wave amplitude will die off as a superposition of damped sinusoids. 

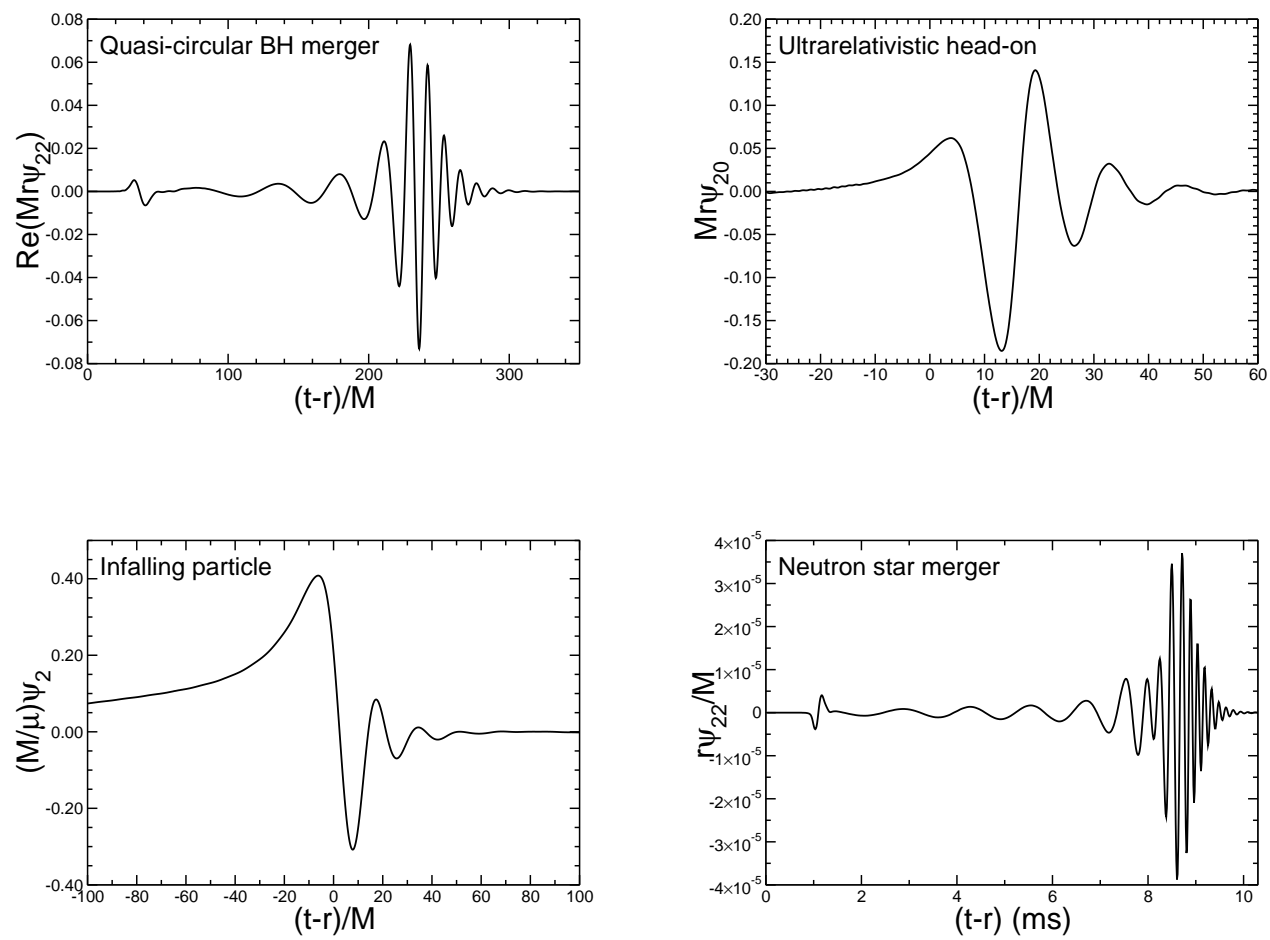

Figure 1. Four different physical processes leading to substantial quasinormal ringing (see text for details). With the exception of the infalling-particle case (where $M$ is the BH mass, $\mu$ the particle's mass and $\psi_{2}$ the Zerilli wavefunction), $\psi_{22}$ is the $l=m=2$ multipolar component of the Weyl scalar $\Psi_{4}, M$ denotes the total mass of the system and $r$ the extraction radius (see e.g. Ref. [44).

Figure 1 shows four different processes involving $\mathrm{BH}$ dynamics. In all of them, quasinormal ringing is clearly visible. The upper-left panel (adapted from Ref. 44]) is the signal from two equal-mass BHs initially on quasi-circular orbits, inspiralling towards each other due to the energy loss induced by gravitational wave emission, merging and forming a single final BH [14. The upper-right panel of Fig. 11 shows gravitational waveforms from numerical simulations of two equal-mass $\mathrm{BHs}$, colliding head-on with $v / c=0.94$ in the center-of-mass frame: as the center-of-mass energy grows (i.e., as the speed of the colliding BHs tends to the speed of light) the waveform is more and more strongly ringdown-dominated [45]. The bottom-left panel shows the gravitational waveform (or more precisely, the dominant, $l=2$ multipole of the Zerilli function) produced by a test particle of mass $\mu$ falling from rest into a Schwarzschild BH [43: the shape of the initial precursor depends on the details of the infall, but the subsequent burst of radiation and the final ringdown are universal features. The bottom-right panel (reproduced from Ref. [46]) shows the waves emitted by two massive neutron stars (NSs) with a polytropic equation of state, inspiralling and eventually collapsing to form a single $\mathrm{BH}$.

QNM frequencies for gravitational perturbations of Schwarzschild and Kerr BHs 
have been computed by many authors. Rather than listing numerical tables of wellknown results, we have set up a web page providing tabulated values of the frequencies and fitting coefficients for the QNMs that are most relevant in gravitational wave astronomy [47]. On this web page, we also provide Mathematica notebooks to compute QNMs of Kerr and asymptotically AdS BHs [4].

\subsection{Milestones}

QNM research has a fifty-year-long history. We find it helpful to provide the reader with a "roadmap" in the form of a chronological list of papers that, in our opinion, have been instrumental to shape the evolution of the field. Our summary is necessarily biased and incomplete, and we apologize in advance for the inevitable omissions. A more complete set of references can be found in the rest of this review.

- 1957 - Regge and Wheeler 48 analyze a special class of gravitational perturbations of the Schwarzschild geometry. This effectively marks the birth of BH perturbation theory a decade before the birth of the BH concept itself. The "one-way membrane" nature of the horizon is not yet fully understood, and the boundary conditions of the problem are not under control.

- 1961 - Newman and Penrose 49] develop a formalism to study gravitational radiation using spin coefficients.

- 1963 - Kerr [50] discovers the mathematical solution of Einstein's field equations describing rotating BHs. In the same year, Schmidt identifies the first quasar ("quasistellar radio source"). Quasars (compact objects with luminosity $\sim 10^{12}$ that of our sun, located at cosmological distance [51]) are now believed to be supermassive BHs (SMBHs), described by the Kerr solution.

- 1964 - The UHURU orbiting X-ray observatory makes the first surveys of the X-ray sky discovering over 300 X-ray "stars", most of which turn out to be due to matter accreting onto compact objects. One of these X-ray sources, Cygnus X-1, is soon accepted as the first plausible stellar-mass $\mathrm{BH}$ candidate (see e.g. [52]).

- 1967 - Wheeler [53, 54 coins the term "black hole" (see the April 2009 issue of Physics Today, and Ref. [55] for a fascinating, first-person historical account).

- 1970 - Zerilli [56, 57] extends the Regge-Wheeler analysis to general perturbations of a Schwarzschild BH. He shows that the perturbation equations can be reduced to a pair of Schrödinger-like equations, and applies the formalism to study the gravitational radiation emitted by infalling test particles.

- 1970 - Vishveshwara [42] studies numerically the scattering of gravitational waves by a Schwarzschild BH: at late times the waveform consists of damped sinusoids (now called "ringdown waves").

- 1971 - Press [58] identifies ringdown waves as the free oscillation modes of the BH.

- 1971 - Davis et al. 43. carry out the first quantitative calculation of gravitational radiation emission within $\mathrm{BH}$ perturbation theory, considering a particle falling radially into a Schwarzschild $\mathrm{BH}$. Quasinormal ringing is excited when the particle crosses the maximum of the potential barrier of the Zerilli equation, which is located at $r \simeq 3 M$ (i.e., close to the unstable circular orbit corresponding to the "light ring"). - 1972 - Goebel [59] points out that the characteristic modes of BHs are essentially gravitational waves in spiral orbits close to the light ring.

- 1973 - Teukolsky [60] decouples and separates the equations for perturbations in the Kerr geometry using the Newman-Penrose formalism [49].

- 1974 - Moncrief 61] introduces a gauge-invariant perturbation formalism. 
- 1975 - Chandrasekhar and Detweiler 62] compute numerically some weakly damped characteristic frequencies. They prove that the Regge-Wheeler and Zerilli potentials have the same spectra.

- 1978 - Cunningham, Price and Moncrief 63, 64, 65 study radiation from relativistic stars collapsing to BHs using perturbative methods. QNM ringing is excited.

- 1979 - Gerlach and Sengupta give a comprehensive and elegant mathematical foundation for gauge-invariant perturbation theory 66, 67.

- 1983 - Chandrasekhar's monograph [22] summarizes the state of the art in BH perturbation theory, elucidating connections between different formalisms.

- 1983 - York [68 attempts to relate the QNM spectrum to Hawking radiation. To our knowledge, this is the first attempt to connect the (purely classical) QNMs with quantum gravity.

- 1983 - Mashhoon [69] suggests to use WKB techniques to compute QNMs. Ferrari and Mashhoon 70, analytically compute QNMs using their connection with bound states of the inverted $\mathrm{BH}$ effective potentials.

- 1985 - Stark and Piran 71 extract gravitational waves from a simulation of rotating collapse to a BH in numerical relativity. QNM excitation is observed, as confirmed by more recent work 72 .

- 1985 - Confirming the validity of Goebel's arguments [59, Mashhoon 73 regards QNMs as waves orbiting around the unstable photon orbit and slowly leaking out, and estimates analytically some QNM frequencies in Kerr-Newman backgrounds.

- 1985 - Schutz and Will 74] develop a WKB approach to compute BH QNMs.

- 1985 - Leaver [75, 76, 77] provides the most accurate method to date to compute BH QNMs using continued fraction representations of the relevant wavefunctions, and discusses their excitation using Green's function techniques.

- 1986 - McClintock and Remillard [78] show that the X-ray nova A0620-00 contains a compact object of mass almost certainly larger than $3 M_{\odot}$, paving the way for the identification of many more stellar-mass BH candidates.

- 1989 - Echeverria 9] estimates the accuracy with which one can estimate the mass and angular momentum of a $\mathrm{BH}$ from QNM observations. The formalism is later improved by Finn $[79$ and substantially refined in Ref. [10, where ringdownbased tests of the no-hair theorem of general relativity are shown to be possible. An Appendix of Ref. [10] provides QNM tables to be used in data analysis and in the interpretation of numerical simulations; these data are now available online [47.

- 1992 - Nollert and Schmidt [80] use Laplace transforms to compute QNMs. Fröman et al. 81] first introduce phase-integral techniques in the context of $\mathrm{BH}$ physics.

- 1993 - Anninos et al. 82 first succeed in simulating the head-on collision of two $\mathrm{BHs}$, and observe QNM ringing of the final $\mathrm{BH}$.

- 1993 - Bachelot and Motet-Bachelot 83 show that a potential with compact support does not cause power-law tails in the evolution of Cauchy data. Subsequently Ching et al. [84, 41] generalize this result to potentials falling off faster than exponentially. - 1996 - Gleiser et al. 85 extend the perturbative formalism to second order and use it to estimate radiation from colliding BHs employing the so-called "close limit" approximation, quantifying the limits of validity of linear perturbation theory [86.

- 1997 - Maldacena [6] formulates the AdS/CFT (Anti-de Sitter/Conformal Field Theory) duality conjecture. Shortly afterward, the papers by Gubser, Klebanov, Polyakov 87] and Witten [88] establish a concrete quantitative recipe for the duality. The AdS/CFT era begins. 
- 1998 - The AdS/CFT correspondence is generalized to non-conformal theories in a variety of approaches (see 35] for a review). The terms "gauge-string duality", "gaugegravity duality" and "holography" appear, referring to these generalized settings.

- 1998 - Flanagan and Hughes 89] show that, under reasonable assumptions and depending on the mass range, the signal-to-noise ratio for ringdown waves is potentially larger than the signal-to-noise ratio for inspiral waves in both Earth-based detectors (such as LIGO) and planned space-based detectors (such as LISA).

- 1998 - Hod [17 uses earlier numerical results by Nollert 90 to conjecture that the real part of highly-damped QNMs is equal to $T \ln 3$ ( $T$ being the Hawking temperature), a conjecture later proven by Motl 91 using the continued fraction method. Hod also proposes a connection between QNMs and Bekenstein's ideas on $\mathrm{BH}$ area quantization.

- 1999 - Creighton [12] describes a search technique for ringdown waveforms in LIGO. - 1999 - Two reviews on QNMs appear: Quasinormal modes of stars and black holes, by Kokkotas and Schmidt 4 and Nollert's Quasinormal modes: the characteristic "sound" of black holes and neutron stars [5.

- 1999 - Horowitz and Hubeny 92 compute QNMs of BHs in AdS backgrounds of various dimensions and relate them to relaxation times in the dual CFTs.

- 2000 - Shibata and Uryu 93 perform the first general relativistic simulation of the merger of two neutron stars. More recent simulations confirm that ringdown is excited when the merger leads to $\mathrm{BH}$ formation [46].

- 2001 - Birmingham, Sachs and Solodukhin 94 point out that QNM frequencies of the $(2+1)$-dimensional Bañados-Teitelboim-Zanelli (BTZ) BH [95] coincide with the poles of the retarded correlation function in the dual $(1+1)$-dimensional CFT.

- 2002 - Baker, Campanelli and Lousto 96 complete the "Lazarus" program to "resurrect" early, unstable numerical simulations of $\mathrm{BH}$ binaries and extend them beyond merger using $\mathrm{BH}$ perturbation theory.

- 2002 - Dreyer 97. proposes to resolve an ambiguity in Loop Quantum Gravity using the highly damped QNMs studied by Hod [17.

- 2002 - Son and Starinets 98 formulate a recipe for computing real-time correlation functions in the gauge-gravity duality. They use the recipe to prove that, in the gauge-gravity duality, QNM spectra correspond to poles of the retarded correlation functions.

- 2002 - QNMs of black branes are computed [99]. The lowest QNM frequencies of black branes in the appropriate conserved charges channels are naturally interpreted as hydrodynamic modes of the dual theory [100.

- 2003 - Motl and Neitzke [101] use a monodromy technique (similar to the phase integral approaches of Ref. [81]) to compute analytically highly damped BH QNMs.

- 2003 - In a series of papers [102, 103, 104, Kodama and Ishibashi extend the ReggeWheeler-Zerilli formalism to higher dimensions.

- 2003 - In one of the rare works on probing quantum aspects of gravity with gauge theory in the context of the gauge-gravity duality (usually, the correspondence is used the other way around), Fidkowski et al. 105 study singularities of BHs by investigating the spacelike geodesics that join the boundaries of the Penrose diagram. The complexified geodesics' properties yield the large-mass QNM frequencies previously found for these BHs. This work is further advanced in Ref. [106] and subsequent publications.

- 2004 - Following Motl and Neitzke [101, Natário and Schiappa analytically compute and classify asymptotic QNM frequencies for $d$-dimensional BHs [107]. 
- 2005 - The LIGO detector reaches design sensitivity [108.

- 2005 - Pretorius 14 achieves the first long-term stable numerical evolution of a BH binary. Soon afterwards, other groups independently succeed in evolving merging $\mathrm{BH}$ binaries using different techniques [15, 16]. The waveforms indicate that ringdown contributes a substantial amount to the radiated energy.

- 2005 - Kovtun and Starinets [109] extend the QNM technique in the gauge-gravity duality to vector and gravitational perturbations using gauge-invariant variables for black brane fluctuations. A classification of the fluctuations corresponding to poles of the stress-energy tensor and current correlators in a dual theory in arbitrary dimension is given. These methods and their subsequent development and application in 110, 111, 112, 113. become a standard approach in computing transport properties of strongly coupled theories from dual gravity.

- 2006-2008 - An analytic computation of the lowest QNM frequency in the shear mode gravitational channel of a generic black brane 114 reveals universality, related to the universality of the shear viscosity to entropy density ratio in dual gauge theories. This and further developments [115, 116 also point to a significance of the QNM spectrum in the context of the $\mathrm{BH}$ membrane paradigm (for a recent review of the membrane paradigm approach, see [117]).

- 2008-2009 - QNM spectra are computed in applications of the gauge-gravity duality to condensed matter theory [37, 38.

\subsection{Notation and conventions}

Unless otherwise and explicitly stated, we use geometrized units where $G=c=1$, so that energy and time have units of length. We also adopt the $(-+++)$ convention for the metric. For reference, the following is a list of symbols that are used often throughout the text.

$d \quad$ Total number of spacetime dimensions (we always consider one timelike and $d-1$ spatial dimensions).

$L \quad$ Curvature radius of $(\mathrm{A}) \mathrm{dS}$ spacetime, related to the negative cosmological constant $\Lambda$ in the Einstein equations $\left(G_{\mu \nu}+\Lambda g_{\mu \nu}=0\right)$ through $L^{2}=\mp(d-2)(d-1) /(2 \Lambda)$. The $-\operatorname{sign}$ is for AdS, + for $\mathrm{dS}$.

$M \quad$ Mass of the BH spacetime.

a Kerr rotation parameter: $a=J / M \in[0, M]$.

$r_{+} \quad$ Radius of the BH's event horizon in the chosen coordinates.

$\omega \quad$ Fourier transform variable. The time dependence of any field is $\sim e^{-i \omega t}$. For stable spacetimes, $\operatorname{Im}(\omega)<0$. Also useful is $\mathfrak{w} \equiv \omega / 2 \pi T$.

$\omega_{R}, \omega_{I} \quad$ Real and imaginary part of the QNM frequencies.

$s \quad$ Spin of the field.

$l \quad$ Integer angular number, related to the eigenvalue $A_{l m}=l(l+d-3)$ of scalar spherical harmonics in $d$ dimensions.

$n \quad$ Overtone number, an integer labeling the QNMs by increasing $|\operatorname{Im}(\omega)|$. We conventionally start counting from a "fundamental mode" with $n=0$. 


\section{A black hole perturbation theory primer}

Within general relativity (and various extensions thereof involving higher-derivative gravity), QNMs naturally appear in the analysis of linear perturbations of fixed gravitational backgrounds. The perturbations obey linear second-order differential equations, whose symmetry properties are dictated by the symmetries of the background. In most cases, these symmetries allow one to separate variables with an appropriate choice of coordinates reducing the system to a set of linear ordinary differential equations (ODEs) or a single ODE. The ODEs are supplemented by boundary conditions, usually imposed at the BH's horizon and at spatial infinity. QNMs are the eigenmodes of this system of equations. The precise choice of the boundary conditions is physically motivated, but it is clear that the presence of the horizon, acting for classical fields as a one-sided membrane, is of crucial importance: it makes the boundary value problem non-hermitian and the associated eigenvalues complex. The methods used to reduce the problem to a single ODE depend on the metric under consideration; some of them are discussed and compared in Chandrasekhar's book 22. Given the progress in the field in recent years and the vast literature on the subject, we will not attempt to describe these techniques in detail. As a simple example illustrating the main extensions of the formalism described in 22] we discuss field perturbations in $d$-dimensional, non-rotating geometries. For the interested reader, Sections [5, 6] and 7 provide references on other background geometries.

\subsection{Perturbations of the Schwarzschild-anti-de Sitter geometry}

Consider the Einstein-Hilbert gravitational action for a $d$-dimensional spacetime with cosmological constant $\Lambda$ :

$$
S=\frac{1}{16 \pi G} \int d^{d} x \sqrt{-g}(R-2 \Lambda)+\int d^{d} x \sqrt{-g} \mathcal{L}_{m},
$$

where $\mathcal{L}_{m}$ is the Lagrangian representing a generic contribution of the "matter fields" (scalar, Maxwell, $p$-form, Dirac and so on) coupled to gravity. The specific form of $\mathcal{L}_{m}$ depends on the particular theory. The Einstein equations read

$$
G_{\mu \nu}+\Lambda g_{\mu \nu}=8 \pi G T_{\mu \nu}
$$

where $T_{\mu \nu}$ is the stress-energy tensor associated with $\mathcal{L}_{m}$. Eq. (21) should be supplemented by the equations of motion for the matter fields. Together with Eq. (2), they form a complicated system of non-linear partial differential equations describing the evolution of all fields including the metric. A particular solution of this system forms a set of background fields $g_{\mu \nu}^{B G}, \Phi^{B G}$, where $\Phi$ is a cumulative notation for all matter fields present. By writing $g_{\mu \nu}=g_{\mu \nu}^{B G}+h_{\mu \nu}, \Phi=\Phi^{B G}+\phi$, and linearizing the full system of equations with respect to the perturbations $h_{\mu \nu}$ and $\phi$, we obtain a set of linear differential equations satisfied by the perturbations.

Maximally symmetric vacuum $\left(T_{\mu \nu}^{B G}=0\right)$ solutions to the field equations are Minkowski, de Sitter (dS) and anti-de Sitter (AdS) spacetimes, depending on the value of the cosmological constant (zero, positive or negative, respectively). Generic solutions of Eq. (2) are asymptotically flat, dS or AdS. We will be mostly interested in asymptotically flat or AdS spacetimes. AdS spacetimes of various dimension arise as a natural groundstate of supergravity theories and as the near-horizon geometry 
of extremal BHs and $p$-branes in string theory, and therefore they play an important role in the AdS/CFT correspondence [35, 118, 119, 120, 121].

BHs in asymptotically AdS spacetimes form a class of solutions interesting from a theoretical point of view and central for the gauge-gravity duality at finite temperature. Their relation to dual field theories is discussed in Section 8 In addition to the simplest Schwarzschild-AdS (SAdS) BH, one finds BHs with toroidal, cylindrical or planar topology [122, 123, 124, 125, 126, 127, as well as the Kerr-NewmanAdS family [128. The standard $\mathrm{BH}$ perturbation theory 22 is easily extended to asymptotically AdS spacetimes [102, 129, 130, 131. For illustration we consider the non-rotating, uncharged $d$-dimensional SAdS (or $\mathrm{SAdS}_{d}$ ) $\mathrm{BH}$ with line element

$$
d s^{2}=-f d t^{2}+f^{-1} d r^{2}+r^{2} d \Omega_{d-2}^{2},
$$

where $f(r)=1+r^{2} / L^{2}-r_{0}^{d-3} / r^{d-3}, d \Omega_{d-2}^{2}$ is the metric of the $(d-2)$-sphere, and the AdS curvature radius squared $L^{2}$ is related to the cosmological constant by $L^{2}=-(d-2)(d-1) / 2 \Lambda$. The parameter $r_{0}$ is proportional to the mass $M$ of the spacetime: $M=(d-2) A_{d-2} r_{0}^{d-3} / 16 \pi$, where $A_{d-2}=2 \pi^{(d-1) / 2} / \Gamma[(d-1) / 2]$. The well-known Schwarzschild geometry corresponds to $L \rightarrow \infty$.

Scalar field perturbations Let us focus, for a start, on scalar perturbations in vacuum. The action for a complex scalar field with a conformal coupling is given by $S_{m} \equiv$ $\int d^{d} x \sqrt{-g} \mathcal{L}_{m}$, where

$$
\mathcal{L}_{m}=-\left(\partial_{\mu} \Phi\right)^{\dagger} \partial^{\mu} \Phi-\frac{d-2}{4(d-1)} \gamma R \Phi^{\dagger} \Phi-m^{2} \Phi^{\dagger} \Phi .
$$

For $\gamma=1, m=0$ the action is invariant under the conformal transformations $g_{\mu \nu} \rightarrow \Omega^{2} g_{\mu \nu}, \Phi \rightarrow \Omega^{1-d / 2} \Phi$, and for $\gamma=0, m=0$ one recovers the usual minimally coupled massless scalar. The equations of motion satisfied by the fields $g_{\mu \nu}$ and (massless) $\Phi$ are

$$
\nabla_{\mu} \nabla^{\mu} \Phi=\frac{d-2}{4(d-1)} \gamma R \Phi, \quad G_{\mu \nu}+\Lambda g_{\mu \nu}=8 \pi G T_{\mu \nu},
$$

where $T_{\mu \nu}$ is quadratic in $\Phi$. Considering perturbations of the fields, $g_{\mu \nu}=g_{\mu \nu}^{B G}+h_{\mu \nu}$ and $\Phi=\Phi^{B G}+\phi$ with $\Phi^{B G}=0$, we observe that the linearized equations of motion for $h_{\mu \nu}$ and $\phi$ decouple, and thus the metric fluctuations $h_{\mu \nu}$ can be consistently set to zero. The background metric satisfies $G_{\mu \nu}^{B G}+\Lambda g_{\mu \nu}^{B G}=0$. We choose $g_{\mu \nu}^{B G}$ to be the $\mathrm{SAdS}_{d}$ metric (3). The scalar fluctuation satisfies the equation

$$
\frac{1}{\sqrt{-g_{\mathrm{BG}}}} \partial_{\mu}\left(\sqrt{-g_{\mathrm{BG}}} g_{\mathrm{BG}}^{\mu \nu} \partial_{\nu} \phi\right)=\frac{d(d-2) \gamma}{4 L^{2}} \phi .
$$

The time-independence and the spherical symmetry of the metric imply the decomposition

$$
\phi(t, r, \theta)=\sum_{l m} e^{-i \omega t} \frac{\Psi_{s=0}(r)}{r^{(d-2) / 2}} Y_{l m}(\theta),
$$

where $Y_{l m}(\theta)$ denotes the $d$-dimensional scalar spherical harmonics, satisfying $\Delta_{\Omega_{d-2}} Y_{l m}=-l(l+d-3) Y_{l m}$, with $\Delta_{\Omega_{d-2}}$ the Laplace-Beltrami operator, and the " $s=0$ " label indicates the spin of the field. Here and in the rest of this paper, for notational simplicity, we usually omit the integral over frequency in the Fourier 
transform. Substituting the decomposition into Eq. (6) we get a radial wave equation for $\Psi_{s=0}(r)$ :

$$
f^{2} \frac{d^{2} \Psi_{s=0}}{d r^{2}}+f f^{\prime} \frac{d \Psi_{s=0}}{d r}+\left(\omega^{2}-V_{s=0}\right) \Psi_{s=0}=0 .
$$

We will see shortly that perturbations with other spins satisfy similar equations. In the particular case of $s=0$, the radial potential $V_{s}$ is given by

$$
V_{s=0}=f\left[\frac{l(l+d-3)}{r^{2}}+\frac{d-2}{4}\left(\frac{(d-4) f}{r^{2}}+\frac{2 f^{\prime}}{r}+\frac{d \gamma}{L^{2}}\right)\right] \text {. }
$$

Finally, if we define a "tortoise" coordinate $r_{*}$ by the relation $d r_{*} / d r=1 / f$, Eq. (8) can be written in the form of a Schrödinger equation with the potential $V_{s}$

$$
\frac{d^{2} \Psi_{s}}{d r_{*}^{2}}+\left(\omega^{2}-V_{s}\right) \Psi_{s}=0
$$

Notice that the tortoise coordinate $r_{*} \rightarrow-\infty$ at the horizon (i.e. as $r \rightarrow r_{+}$), but its behavior at infinity is strongly dependent on the cosmological constant: $r_{*} \rightarrow+\infty$ for asymptotically-flat spacetimes, and $r_{*} \rightarrow$ constant for the $\mathrm{SAdS}_{d}$ geometry.

Electromagnetic, gravitational and half-integer spin perturbations Equations for linearized Maxwell field perturbations in curved spacetimes can be obtained along the lines of the scalar field example above. To separate the angular dependence we now need vector spherical harmonics [5, 132, 133. In $d=4$, electromagnetic perturbations can be completely characterized by the wave equation (10) with the potential

$$
V_{s=1}^{d=4}=f\left[\frac{l(l+1)}{r^{2}}\right] .
$$

A comprehensive treatment of the four-dimensional case can be found in Ref. 132 for the Schwarzschild spacetime, and in Ref. [130] for the SAdS geometry. Higherdimensional perturbations are discussed in Ref. [134].

The classification of gravitational perturbations $h_{\mu \nu}(x)$ on a fixed background $g_{\mu \nu}^{B G}(x)$ is more complicated. We focus on the $\mathrm{SAdS}_{4}$ geometry. After a decomposition in tensorial spherical harmonics, the perturbations fall into two distinct classes: odd (Regge-Wheeler or vector-type) and even (Zerilli or scalar-type), with parities equal to $(-1)^{l+1}$ and $(-1)^{l}$, respectively [27, 21, [57, 135]. In the Regge-Wheeler gauge [4, 5, 27, 48, 136], the perturbations are written as $h_{\mu \nu}=e^{-i \omega t} \tilde{h}_{\mu \nu}$, where for odd parity

$$
\tilde{h}_{\mu \nu}=\left[\begin{array}{cccc}
0 & 0 & 0 & h_{0}(r) \\
0 & 0 & 0 & h_{1}(r) \\
0 & 0 & 0 & 0 \\
h_{0}(r) & h_{1}(r) & 0 & 0
\end{array}\right]\left(\sin \theta \frac{\partial}{\partial \theta}\right) Y_{l 0}(\theta)
$$

whereas for even parity

$$
\tilde{h}_{\mu \nu}=\left[\begin{array}{cccc}
H_{0}(r) f & H_{1}(r) & 0 & 0 \\
H_{1}(r) & H_{2}(r) / f & 0 & 0 \\
0 & 0 & r^{2} K(r) & 0 \\
0 & 0 & 0 & r^{2} K(r) \sin ^{2} \theta
\end{array}\right] Y_{l 0}(\theta) .
$$

The angular dependence of the perturbations is dictated by the structure of tensorial spherical harmonics [27, 21, 57, 135. Inserting this decomposition into Einstein's 
equations one gets ten coupled second-order differential equations that fully describe the perturbations: three equations for the odd radial variables, and seven for the even variables. The odd perturbations can be combined in a single Regge-Wheeler or vector-type gravitational variable $\Psi_{s=2}^{-}$, and the even perturbations can likewise be combined in a single Zerilli or scalar-type gravitational wavefunction $\Psi_{s=2}^{+}$. The ReggeWheeler and Zerilli functions $\left(\Psi_{s=2}^{-}\right.$and $\Psi_{s=2}^{+}$, respectively) satisfy the Schrödingerlike equation (10) with the potentials

$$
V_{s=2}^{-}=f(r)\left[\frac{l(l+1)}{r^{2}}-\frac{6 M}{r^{3}}\right]
$$

and

$$
V_{s=2}^{+}=\frac{2 f(r)}{r^{3}} \frac{9 M^{3}+3 \lambda^{2} M r^{2}+\lambda^{2}(1+\lambda) r^{3}+9 M^{2}\left(\lambda r+\frac{r^{3}}{L^{2}}\right)}{(3 M+\lambda r)^{2}} .
$$

The parameters $h_{0}$ and $h_{1}$ of the vector-type perturbation are related to $\Psi_{s=2}^{-}$by

$$
\Psi_{s=2}^{-}=\frac{f(r)}{r} h_{1}(r), \quad h_{0}=\frac{i}{\omega} \frac{d}{d r_{*}}\left(r \Psi_{s=2}^{-}\right) .
$$

For the scalar-type gravitational perturbation, the functions $H_{1}$ and $K$ can be expressed through $\Psi_{s=2}^{+}$via

$$
\begin{aligned}
K & =\frac{6 M^{2}+\lambda(1+\lambda) r^{2}+3 M\left(\lambda r-\frac{r^{3}}{L^{2}}\right)}{r^{2}(3 M+\lambda r)} \Psi_{s=2}^{+}+\frac{d \Psi_{s=2}^{+}}{d r_{*}}, \\
H_{1} & =\frac{i \omega\left(3 M^{2}+3 \lambda M r-\lambda r^{2}+3 M \frac{r^{3}}{L^{2}}\right)}{r(3 M+\lambda r) f(r)} \Psi_{s=2}^{+}-\frac{i \omega r}{f(r)} \frac{d \Psi_{s=2}^{+}}{d r_{*}},
\end{aligned}
$$

where $\lambda \equiv(l-1)(l+2) / 2$, and $H_{0}$ is then obtained from the algebraic relation

$$
\begin{aligned}
& {\left[(l-1)(l+2)+\frac{6 M}{r}\right] H_{0}+\left[i \frac{l(l+1)}{\omega r^{2}}\left(M+r^{3} / L^{2}\right)-2 i \omega r\right] H_{1} } \\
- & {\left[(l-1)(l+2)+r f^{\prime}-\frac{4 \omega^{2} r^{2}+r^{2} f^{\prime 2}}{2 f}\right] K=0 . }
\end{aligned}
$$

A complete discussion of Regge-Wheeler or vector-type gravitational perturbations of the four-dimensional Schwarzschild geometry can be found in the original papers by Regge and Wheeler [48 as well as in Ref. [137, where some typos in the original work are corrected. For Zerilli or scalar-type gravitational perturbations, the fundamental reference is Zerilli's work [56, 57]; typos are corrected in Appendix A of Ref. 138. An elegant, gauge-invariant decomposition of gravitational perturbations of the Schwarzschild geometry is described by Moncrief 61] (see also 66, 67, 139, 140]). These papers are reviewed by Nollert [5] and Nagar and Rezzolla [27. For an alternative treatment, see Chandrasekhar's book 22. Chandrasekhar's book and papers 141, 142 use a different notation, exploring mathematical aspects of the relations between different gravitational perturbations (see Appendix A). Extensions to the $\mathrm{SAdS}_{4}$ geometry can be found in Ref. [130, while the general $d$-dimensional case has been explored in a series of papers by Kodama and Ishibashi [102, 103, 104.

The case of Dirac fields seems to have been discussed first by Brill and Wheeler [143], with important extensions of the formalism by Page [144], Unruh [145] and Chandrasekhar [22]. For the treatment of Rarita-Schwinger fields, see [146]. 
To summarize this Section: in four-dimensional Schwarzschild or SAdS backgrounds, scalar $(m=0, \gamma=0, s=0)$, electromagnetic $(s= \pm 1)$ and ReggeWheeler or vector-type gravitational $(s=2)$ perturbations, can be described by the master equation (10) with the potential

$$
V_{s}=f\left[\frac{l(l+1)}{r^{2}}+\left(1-s^{2}\right)\left(\frac{2 M}{r^{3}}+\frac{4-s^{2}}{2 L^{2}}\right)\right] .
$$

The potentials for the scalar-type gravitational perturbations and half-integer spin perturbations have forms different from (20), see for instance Refs. [102, 147. However, the vector-type (Regge-Wheeler) and scalar-type (Zerilli) potentials have the remarkable property of being isospectral, i.e. they possess the same QNM spectrum. The origin of this isospectrality, first discovered by Chandrasekhar [22, is reviewed in Appendix A

\subsection{Higher-dimensional gravitational perturbations}

The literature on gravitational perturbations can be quite confusing. Naming conventions were already unclear in 1970, so much so that Zerilli decided to list equivalent terminologies referring to odd and even tensor spherical harmonics (cf. Table II of Ref. [57]). The situation got even worse since then. Chandrasekhar's book, which is the most complete reference in the field, established a different terminology: "odd" (Regge-Wheeler) perturbations were called "axial" and described by a master variable $\Psi^{-}$, while "even" (Zerilli) perturbations were renamed "polar" and described by a master variable $\Psi^{+}$. In recent years, Kodama and Ishibashi 102, 103. 104 extended the gauge-invariant perturbation framework to higherdimensional, non-rotating BHs. In higher dimensions three master variables are necessary to completely describe the perturbations 102. Two of them (the vectortype gravitational perturbations and the scalar-type gravitational perturbations) reduce to the Regge-Wheeler and Zerilli master variables in $d=4$. Kodama and Ishibashi refer to the third type of perturbations, which have no four-dimensional analogue, as tensor-type gravitational perturbations. In this review we will usually adopt the Kodama-Ishibashi terminology.

\subsection{Weak fields in the Kerr background: the Teukolsky equation}

In four-dimensional asymptotically flat spacetimes, the most general vacuum $\mathrm{BH}$ solution of Einstein's equations is the Kerr metric. In the standard Boyer-Linquist coordinates, the metric depends on two parameters: the mass $M$ and spin $J=a M$. The spacetime has a Cauchy horizon at $r=r_{-}=M-\sqrt{M^{2}-a^{2}}$ and an event horizon at $r=r_{+}=M+\sqrt{M^{2}-a^{2}}$. The separation of variables for a minimally coupled scalar field in the Kerr background was first reported by Brill et al. 148.

Teukolsky 60, 149] showed that if one works directly in terms of curvature invariants, the perturbation equations decouple and separate for all Petrov type-D spacetimes. He derived a master perturbation equation governing fields of general spin, including the most interesting gravitational perturbations (see [22, 150] for reviews). Teukolsky's approach is based on the Newman-Penrose [49] formalism. In this formalism one introduces a tetrad of null vectors $\mathbf{l}, \mathbf{n}, \mathbf{m}, \mathbf{m}^{*}$ at each point in spacetime, onto which all tensors are projected. The Newman-Penrose equations are relations linking the tetrad vectors, the spin coefficients, the Weyl tensor, the Ricci tensor and the scalar curvature [49]. The most relevant perturbation variables, which 
both vanish in the background spacetime, are the Weyl scalars $\boldsymbol{\Psi}_{0}$ and $\boldsymbol{\Psi}_{4}$, obtained by contracting the Weyl tensor $C_{\mu \nu \lambda \sigma}$ [151] on the tetrad legs (roughly speaking, these quantities describe ingoing and outgoing gravitational radiation):

$$
\begin{aligned}
& \boldsymbol{\Psi}_{0}=-C_{1313}=-C_{\mu \nu \lambda \sigma} l^{\mu} m^{\nu} l^{\lambda} m^{\sigma}, \\
& \boldsymbol{\Psi}_{4}=-C_{2424}=-C_{\mu \nu \lambda \sigma} n^{\mu} m^{* \nu} n^{\lambda} m^{* \sigma} .
\end{aligned}
$$

Two analogous quantities $\boldsymbol{\Phi}_{0}$ and $\boldsymbol{\Phi}_{2}$ describe electromagnetic perturbations:

$$
\boldsymbol{\Phi}_{0}=F_{\mu \nu} l^{\mu} m^{\nu}, \quad \boldsymbol{\Phi}_{2}=F_{\mu \nu} m^{* \mu} n^{\nu} .
$$

By Fourier-transforming a spin-s field $\psi(t, r, \theta, \phi)$ and expanding it in spin-weighted spheroidal harmonics as follows:

$$
\psi(t, r, \theta, \phi)=\frac{1}{2 \pi} \int e^{-i \omega t} \sum_{l=|s|}^{\infty} \sum_{m=-l}^{l} e^{i m \phi}{ }_{s} S_{l m}(\theta) R_{l m}(r) d \omega,
$$

Teukolsky finds separated ODEs for ${ }_{s} S_{l m}$ and $R_{l m}$ [60, 149]:

$$
\begin{aligned}
& {\left[\frac{\partial}{\partial u}\left(1-u^{2}\right) \frac{\partial}{\partial u}\right]{ }_{s} S_{l m} } \\
+ & {\left[a^{2} \omega^{2} u^{2}-2 a \omega s u+s+{ }_{s} A_{l m}-\frac{(m+s u)^{2}}{1-u^{2}}\right]{ }_{s} S_{l m}=0, } \\
& \Delta \partial_{r}^{2} R_{l m}+(s+1)(2 r-2 M) \partial_{r} R_{l m}+V R_{l m}=0 .
\end{aligned}
$$

Here $u \equiv \cos \theta, \Delta=\left(r-r_{-}\right)\left(r-r_{+}\right)$and

$$
\begin{aligned}
V= & 2 i s \omega r-a^{2} \omega^{2}-{ }_{s} A_{l m}+\frac{1}{\Delta}\left[\left(r^{2}+a^{2}\right)^{2} \omega^{2}-4 M a m \omega r+a^{2} m^{2}\right. \\
& \left.+i s\left(a m(2 r-2 M)-2 M \omega\left(r^{2}-a^{2}\right)\right)\right]
\end{aligned}
$$

The solutions to the angular equation (25) are known in the literature as spinweighted spheroidal harmonics: ${ }_{s} S_{l m}={ }_{s} S_{l m}(a \omega, \theta, \phi)$. For $a \omega=0$ the spinweighted spheroidal harmonics reduce to spin-weighted spherical harmonics ${ }_{s} Y_{l m}(\theta, \phi)$ 152. In this case the angular separation constants ${ }_{s} A_{l m}$ are known analytically: ${ }_{s} A_{l m}(a=0)=l(l+1)-s(s+1)$. The determination of the angular separation constant in more general cases is a non-trivial problem (see [153] and references therein).

Table 1. Teukolsky wavefunction $\psi$, as in (24), for each value $s$ of the spin. The spin-coefficient $\rho \equiv-1 /(r-i a \cos \theta)$. The quantities $\chi_{0}$ and $\chi_{1}$ refer to the components of the neutrino wavefunction along dyad legs.

\begin{tabular}{|c||cccc|}
\hline$s$ & 0 & $(+1 / 2,-1 / 2)$ & $(+1,-1)$ & $(+2,-2)$ \\
$\psi$ & $\Phi$ & $\left(\chi_{0}, \rho^{-1} \chi_{1}\right)$ & $\left(\boldsymbol{\Phi}_{0}, \rho^{-2} \boldsymbol{\Phi}_{2}\right)$ & $\left(\mathbf{\Psi}_{0}, \rho^{-4} \mathbf{\Psi}_{4}\right)$ \\
\hline
\end{tabular}

The field's spin weight $s$ is equal to $0, \pm 1 / 2, \pm 1, \pm 2$ for scalar, Dirac, electromagnetic and gravitational perturbations, respectively. The Teukolsky master variable $\psi$ is related to the perturbation fields by the relations listed in Table 1 (see also Appendix B of Ref. [149]). Relations between the Regge-Wheeler-Zerilli and the Teukolsky variables are explored in Ref. 22]. Reconstructing the metric from the Teukolsky functions is a highly non-trivial problem which is still not completely solved (see e.g. [154, 155, 156, 157, 158, 159]). 


\section{Defining quasinormal modes}

\subsection{Quasinormal modes as an eigenvalue problem}

In a spherically symmetric background, the study of $\mathrm{BH}$ perturbations due to linearized fields of spin $s$ can be reduced to the study of the differential equation (10). Henceforth, to simplify the notation, we will usually drop the $s$-subscript in all quantities. To determine the free modes of oscillation of a $\mathrm{BH}$, which correspond to "natural" solutions of this unforced ODE, we must impose physically appropriate boundary conditions at the horizon $\left(r_{*} \rightarrow-\infty\right)$ and at spatial infinity $\left(r_{*} \rightarrow \infty\right)$. These boundary conditions are discussed below.

Boundary conditions at the horizon For most spacetimes of interest the potential $V \rightarrow 0$ as $r_{*} \rightarrow-\infty$, and in this limit solutions to the wave equation (10) behave as $\Psi \sim e^{-i \omega\left(t \pm r_{*}\right)}$. Classically nothing should leave the horizon: only ingoing modes (corresponding to a plus sign) should be present, and therefore

$$
\Psi \sim e^{-i \omega\left(t+r_{*}\right)}, \quad r_{*} \rightarrow-\infty\left(r \rightarrow r_{+}\right) .
$$

This boundary condition at the horizon can also be seen to follow from regularity requirements. For non-extremal spacetimes, the tortoise coordinate tends to

$$
r_{*}=\int f^{-1} d r \sim\left[f^{\prime}\left(r_{+}\right)\right]^{-1} \log \left(r-r_{+}\right), \quad r \sim r_{+},
$$

with $f^{\prime}\left(r_{+}\right)>0$. Near the horizon, outgoing modes behave as

$$
e^{-i \omega\left(t-r_{*}\right)}=e^{-i \omega v} e^{2 i \omega r_{*}} \sim e^{-i \omega v}\left(r-r_{+}\right)^{2 i \omega / f^{\prime}\left(r_{+}\right)},
$$

where $v=t+r_{*}$. Now Eq. (29) shows that unless $2 i \omega / f^{\prime}\left(r_{+}\right)$is a positive integer the outgoing modes cannot be smooth, i.e. of class $\mathcal{C}^{\infty}$, and they must be discarded. An elegant discussion of the correct boundary conditions at the horizon of rotating BHs can be found in Appendix B of Ref. [160].

Boundary conditions at spatial infinity: asymptotically flat spacetimes For asymptotically flat spacetimes, the metric at spatial infinity tends to the Minkowski metric. From Eq. (20) with $L \rightarrow \infty$ we see that the potential is zero at infinity. By requiring

$$
\Psi \sim e^{-i \omega\left(t-r_{*}\right)}, \quad r \rightarrow \infty,
$$

we discard unphysical waves "entering the spacetime from infinity".

The main difference between QNM problems and other prototypical physical problems involving small oscillations, such as the vibrating string, is that the system is now dissipative: waves can escape either to infinity or into the BH. For this reason an expansion in normal modes is not possible [4, 5, 77, 80. There is a discrete infinity of QNMs, defined as eigenfunctions satisfying the above boundary conditions. The corresponding eigenfrequencies $\omega_{\mathrm{QNM}}$ have both a real and an imaginary part, the latter giving the (inverse) damping time of the mode. One usually sorts the QNM frequencies by the magnitude of their imaginary part, and labels them by an integer $n$ called the overtone number. The fundamental mode $n=0$ is the least damped mode, and being very long-lived it usually dominates the ringdown waveform.

A seemingly pathological behavior occurs when one imposes the boundary conditions (27) and (30). When the mode amplitude decays in time, the characteristic frequency $\omega_{\mathrm{QNM}}$ must have a negative imaginary component. Then, the amplitude 
near infinity $\left(r_{*} \rightarrow+\infty\right)$ must blow up. So it is in general impossible to represent regular initial data on the spacetime as a sum of QNMs. QNMs should be thought of as quasistationary states which cannot have existed for all times: they decay exponentially with time, and are excited only at a particular instant in time (see 161 for an alternative viewpoint on "dynamic" QNM excitation). In more formal terms, QNMs do not form a complete set of wavefunctions [5].

Boundary conditions at spatial infinity: asymptotically anti-de Sitter spacetimes When the cosmological constant does not vanish, by inspection of Eq. (10) we see that

$$
\Psi_{s=0} \sim A r^{-2}+B r, \quad \Psi_{s=1,2} \sim A / r+B, \quad r \rightarrow \infty .
$$

Regular scalar field perturbations should have $B=0$, corresponding to Dirichlet boundary conditions at infinity. The case for electromagnetic and gravitational perturbations is less clear: there is no a priori compelling reason for a specific boundary condition. A popular choice implements Dirichlet boundary conditions for the ReggeWheeler and Zerilli variables [130, but other boundary conditions were investigated, e.g., in Ref. [162]. A discussion of preferred boundary conditions in the context of the AdS/CFT correspondence can be found in Refs. [163, 164, 165] (see also Section 8.2).

\subsection{Quasinormal modes as poles in the Green's function}

The QNM contribution to the $\mathrm{BH}$ response to a generic perturbation can be identified formally by considering the Green's function solution to an inhomogeneous wave equation [77, 80, 166, 161, 167. Consider the Laplace transform of the field, $\mathcal{L} \Psi(t, r) \equiv \Psi(\omega, r)=\int_{t_{0}}^{\infty} \Psi(t, r) e^{i \omega t} d t$, which is well defined if $\omega_{I} \geq c$ (the usual Laplace variable is $s=-i \omega$; we use $\omega$ for notational consistency with previous works). The problem of computing the gravitational waveform produced when a $\mathrm{BH}$ is perturbed by some material source (such as a particle of mass $m \ll M$ falling into the $\mathrm{BH}$ ) can be reduced to a wave equation of the form (10) with a source term:

$$
\frac{d^{2} \Psi}{d r_{*}^{2}}+\left(\omega^{2}-V\right) \Psi=I(\omega, r) \text {. }
$$

We can solve this equation by the standard Green's function technique [168] (see Refs. [77, 80, 166, 161, 167] for applications in this context), focusing for definiteness on asymptotically flat spacetimes. Take two independent solutions of the homogeneous equation: one has the correct behavior at the horizon,

$$
\begin{aligned}
& \lim _{r \rightarrow r_{+}} \Psi_{r_{+}} \sim e^{-i \omega r_{*}}, \\
& \lim _{r \rightarrow \infty} \Psi_{r_{+}} \sim A_{\text {in }}(\omega) e^{-i \omega r_{*}}+A_{\text {out }}(\omega) e^{i \omega r_{*}},
\end{aligned}
$$

and the second independent solution $\Psi_{\infty_{+}} \sim e^{i \omega r_{*}}$ for large $r$. The Wronskian of these two wavefunctions is $W=2 i \omega A_{\text {in }}$, and we can express the general solution as 77 ]

$$
\Psi(\omega, r)=\Psi_{\infty_{+}} \int_{-\infty}^{r_{*}} \frac{I(\omega, r) \Psi_{r_{+}}}{2 i \omega A_{\mathrm{in}}} d r_{*}^{\prime}+\Psi_{r_{+}} \int_{r_{*}}^{\infty} \frac{I(\omega, r) \Psi_{\infty_{+}}}{2 i \omega A_{\mathrm{in}}} d r_{*}^{\prime} .
$$

The time-domain response is obtained by inversion of the Laplace transform:

$$
\Psi(t, r)=\frac{1}{2 \pi} \int_{-\infty+i c}^{\infty+i c} \Psi(\omega, r) e^{-i \omega t} d \omega
$$




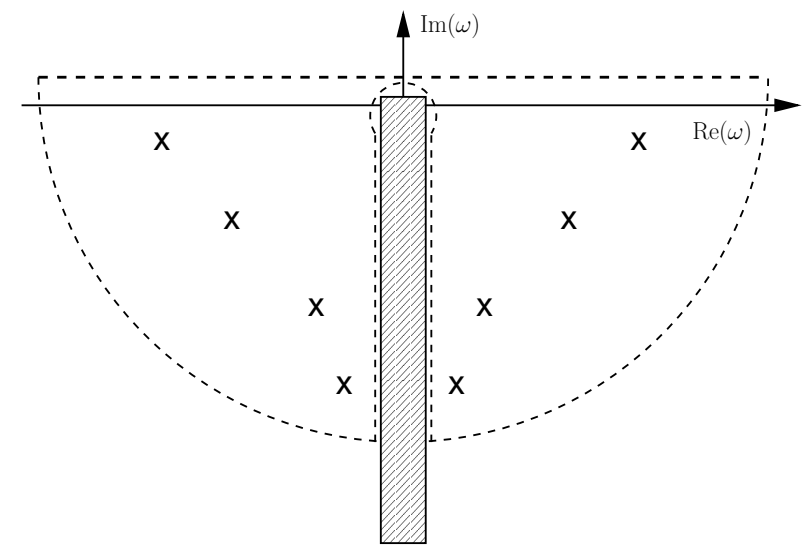

Figure 2. Integration contour for Eq. (36). The hatched area is the branch cut and crosses mark zeros of the Wronskian $W$ (the QNM frequencies).

The frequency integral can be performed by the integration contour shown in Fig. 2 , There are in general three different contributions to the integral. The integral along the large quarter-circles is the flat-space analogue of the prompt response, i.e. waves propagating directly from the source to the observer at the speed of light. Depending on the asymptotic structure of the potential, there is usually a branch point at $\omega=0$ [41. To prevent it from lying inside the integration contour, we place a branch cut along the negative imaginary- $\omega$ axis and split the half circle at $|\omega| \rightarrow \infty$ into two quarter circles [169, 170, 84]. The branch-cut contribution gives rise to late-time tails [171, 77, 84]: physically, these tails are due to backscattering off the background curvature [171, and therefore they depend on the asymptotics of the spacetime. Tails are absent for certain backgrounds, such as the SAdS [92] or the Nariai spacetime [172]. The third contribution comes from a sum-over-residues at the poles in the complex frequency plane, which are the zeros of $A_{\text {in }}$. These poles correspond to perturbations satisfying both in-going wave conditions at the horizon and out-going wave conditions at infinity, so (by the very definition of QNMs) they represent the QNM contribution to the response. Although we used the asymptotic behavior of the solutions of the wave equation, this discussion can be trivially generalized to any spacetime.

Far from the source, the QNM contribution in asymptotically flat spacetimes can be written as [166, 167]

$$
\Psi(t, r)=-\operatorname{Re}\left[\sum_{n} C_{n} e^{-i \omega_{n}\left(t-r_{*}\right)}\right],
$$

where the sum is over all poles in the complex plane. The $C_{n}$ 's are called quasinormal excitation coefficients and they quantify the QNM content of the waveform. They are related to initial-data independent quantities, called the quasinormal excitation factors (QNEFs) and denoted by $B_{n}$, as follows:

$$
C_{n}=B_{n} \int_{-\infty}^{\infty} \frac{I(\omega, r) \Psi_{r_{+}}}{A_{\text {out }}} d r_{*}^{\prime}, \quad B_{n}=\left.\frac{A_{\text {out }}}{2 \omega}\left(\frac{d A_{\text {in }}}{d \omega}\right)^{-1}\right|_{\omega=\omega_{n}} .
$$

In general the QNM frequencies $\omega_{n}$, the $B_{n}$ 's and the $C_{n}$ 's depend on $l, m$ and on the spin of the perturbing field $s$, but to simplify the notation we will omit this dependence 
whenever there is no risk of confusion.

The QNEFs play an important role in BH perturbation theory: they depend only on the background geometry, and (when supplemented by specific initial data) they allow the determination of the QNM content of a signal, i.e. of the $C_{n}$ 's. This has been known for over two decades, but relatively little effort has gone into understanding how these modes are excited by physically relevant perturbations. The QNEFs have long been known for scalar, electromagnetic and gravitational perturbations of Schwarzschild BHs 77, 173, 166, 161, and they have recently been computed for general perturbations of Kerr BHs [167. In [167] it was also shown that for large overtone numbers $(n \rightarrow \infty) B_{n} \propto n^{-1}$ for all perturbing fields in a large class of non-rotating spacetimes. The excitation factors $C_{n}$ have mainly been computed for the simple case where the initial data are Gaussian pulses of radiation [166, 167. The only work we are aware of studying the excitation factors $C_{n}$ for a point particle falling into a Schwarzschild BH is Leaver's classic paper 77 .

Besides the theoretical interest of quantifying QNM excitation by generic initial data in the framework of perturbation theory, excitation factors and excitation coefficients have useful applications in gravitational wave data analysis. First of all, a formal QNM expansion of the BH response can simplify the calculation of the self-force acting on small bodies orbiting around BHs. It was shown recently, using as a model problem the Nariai spacetime, that a QNM expansion of the Green's function can be used for a matched expansion of the "quasi-local" and "distant-past" contributions to the self force [172. The problem of quantifying QNM excitation is of paramount importance to search for inspiralling compact binaries in gravitational wave detector data. All attempts to match an effective-one-body description of inspiralling binaries to numerical relativity simulations found that the inclusion of several overtones in the ringdown waveform is a crucial ingredient to improve agreement with the numerics 174, 175. So far the matching of the inspiral and ringdown waveforms has been performed by ad hoc procedures. For example, the amplitudes and phases of the $C_{n}$ 's have been fixed by requiring continuity of the waveform on a grid of points, or "comb" [176]. These matching procedures have their own phenomenological interest, but a self-consistent estimation of the excitation coefficients within perturbation theory is needed to improve our physical understanding of the inspiral-ringdown transition.

Finally, we point out that recent investigations have addressed the issue of mode excitation in the gauge/gravity duality [177, 178. There it was found, for example, that the residue of the diffusion and shear mode decays at small wavelength, so these modes effectively cease to exist.

\section{Computing quasinormal modes}

To determine the QNMs and compute their frequencies we must solve the eigenvalue problem represented by the wave equation (10), with boundary conditions specified by Eq. (27) at the horizon and Eq. (30) at infinity. There is no universal prescription to compute QNMs. In this section we discuss various methods to obtain such a solution, pointing out that different methods are better suited to different spacetimes.

For a start we consider the exceptional cases where an exact, analytical solution to the wave equation can be found. In some spacetimes the potential appearing in the wave equation can be shown to reduce to the Pöschl-Teller potential [179], for which an exact QNM calculation is possible [70]. These spacetimes and their QNM spectra are reviewed in Section 4.1. In the general case, QNM calculations require approximations 
or numerical methods $\$$. Some of these (including WKB approximations, monodromy methods, series solutions in asymptotically AdS backgrounds and Leaver's continued fraction method) are reviewed in Sections 4.2, 4.6.

\subsection{Exact solutions}

In general exact solutions to the wave equation are hard to find, and they must be computed numerically. There are a few noteworthy exceptions, some of which we summarize here.

We begin our review of exact solutions by sketching the analytical derivation of the QNMs of the Pöschl-Teller potential. Many of the difficulties in computing QNMs in $\mathrm{BH}$ spacetimes arise from the slow decay of the potential as $r \rightarrow \infty$, which is due (mathematically) to the presence of a branch cut and gives rise (physically) to backscattering of gravitational waves off the gravitational potential and to late-time tails. Ferrari and Mashhoon realized that these difficulties can be removed and exact solutions can be found if one considers instead a potential that decays exponentially as $r \rightarrow \infty$, while recovering the other essential features of the Schwarzschild potential. Such a potential is the Pöschl-Teller potential [70]. After reviewing QNM solutions for the Pöschl-Teller potential we briefly review the modes of pure AdS and dS spacetimes. Then we show that perturbations of the near-extreme Schwarzschild deSitter (SdS) and of the Nariai spacetime reduce to a wave equation with a Pöschl-Teller potential, so they can be solved analytically. We also discuss two asymptotically AdS BH spacetimes which have improved our understanding of the role of QNMs in the AdS/CFT correspondence: the $(2+1)$-dimensional BTZ BH 184 and the $d$-dimensional topological (massless) BH [122, 123, 124, 125, 126, 127. The BTZ BH is the first $\mathrm{BH}$ spacetime for which an exact, analytic expression for the QNMs has been derived 95, and it offers interesting insights into the validity of the AdS/CFT correspondence [94, 185, 186].

The Pöschl-Teller potential In this section we analytically compute the QNMs of the Pöschl-Teller potential [179, 170, which will serve as a prototype for several $\mathrm{BH}$ spacetimes to be discussed below. Consider the equation

$$
\frac{\partial^{2} \Psi}{\partial r_{*}^{2}}+\left[\omega^{2}-\frac{V_{0}}{\cosh ^{2} \alpha\left(r_{*}-\bar{r}_{*}\right)}\right] \Psi=0 .
$$

The quantity $\bar{r}_{*}$ is the point $r_{*}$ at which the potential attains a maximum, i.e. $d V / d r_{*}\left(\bar{r}_{*}\right)=0$, and $V_{0}$ is the value of the Pöschl-Teller potential at that point: $V_{0}=V\left(\bar{r}_{*}\right)$. The quantity $\alpha$ is related to the second derivative of the potential at $r_{*}=\bar{r}_{*}, \alpha^{2} \equiv-\left(2 V_{0}\right)^{-1} d^{2} V / d r_{*}^{2}\left(\bar{r}_{*}\right)$. The solutions of Eq. (39) that satisfy both boundary conditions (27) and (30) are the QNMs [70. To find these solutions we define a new independent variable $\xi=\left[1+e^{-2 \alpha\left(r_{*}-\bar{r}_{*}\right)}\right]^{-1}$ and rewrite Eq. (39) as

$$
\xi^{2}(1-\xi)^{2} \frac{\partial^{2} \Psi}{\partial \xi^{2}}-\xi(1-\xi)(2 \xi-1) \frac{\partial \Psi}{\partial \xi}+\left[\frac{\omega^{2}}{4 \alpha^{2}}-\frac{V_{0}}{\alpha^{2}} \xi(1-\xi)\right] \Psi=0 \text {. }
$$

$\ddagger$ Fiziev [180, 181 actually showed that the Regge-Wheeler and Teukolsky equations can be solved analytically in terms of confluent Heun's functions. These solutions allow a high-accuracy determination of the Schwarzschild quasinormal frequencies. The calculation of Heun's functions is quite contrived, but it already proved useful to address interesting open problems in perturbation theory 182183 . 
Near spatial infinity $1-\xi \sim e^{-2 \alpha\left(r_{*}-\bar{r}_{*}\right)}$, and near the horizon $\xi \sim e^{2 \alpha\left(r_{*}-\bar{r}_{*}\right)}$. If we define $a=\left[\alpha+\sqrt{\alpha^{2}-4 V_{0}}-2 i \omega\right] /(2 \alpha), b=\left[\alpha-\sqrt{\alpha^{2}-4 V_{0}}-2 i \omega\right] /(2 \alpha), c=$ $1-i \omega / \alpha$ and set $\Psi=(\xi(1-\xi))^{-i \omega /(2 \alpha)} y$, we get a standard hypergeometric equation for $y$ [187]:

$$
\xi(1-\xi) \partial_{\xi}^{2} y+[c-(a+b+1) \xi] \partial_{\xi} y-a b y=0
$$

and therefore

$$
\begin{aligned}
\Psi & =A \xi^{i \omega /(2 \alpha)}(1-\xi)^{-i \omega /(2 \alpha)} F(a-c+1, b-c+1,2-c, \xi) \\
& +B(\xi(1-\xi))^{-i \omega /(2 \alpha)} F(a, b, c, \xi) .
\end{aligned}
$$

Recalling that $F\left(a_{1}, a_{2}, a_{3}, 0\right)=1$ and that $\xi^{i \omega /(2 \alpha)} \sim e^{i \omega r_{*}}$ near the horizon, we see that the first term represents, according to our conventions, an outgoing wave at the horizon, while the second term represents an ingoing wave. QNM boundary conditions require $A=0$. To investigate the behavior at infinity, one uses the $z \rightarrow 1-z$ transformation law for the hypergeometric function [187]:

$$
\begin{aligned}
F(a, b, c, z) & =(1-z)^{c-a-b} \frac{\Gamma(c) \Gamma(a+b-c)}{\Gamma(a) \Gamma(b)} F(c-a, c-b, c-a-b+1,1-z) \\
& +\frac{\Gamma(c) \Gamma(c-a-b)}{\Gamma(c-a) \Gamma(c-b)} F(a, b,-c+a+b+1,1-z) .
\end{aligned}
$$

The boundary condition at infinity implies either $1 / \Gamma(a)=0$ or $1 / \Gamma(b)=0$, which are satisfied whenever

$$
\omega= \pm \sqrt{V_{0}-\alpha^{2} / 4}-i \alpha(2 n+1) / 2, \quad n=0,1,2, \ldots
$$

where $n$ is the overtone index.

A popular approximation scheme to compute BH QNMs consists in replacing the true potential in a given spacetime by the Pöschl-Teller potential. This approximation works well for the low-lying modes of the Schwarzschild geometry. It predicts $M \omega=0.1148-0.1148 i, 0.3785-0.0905 i$ for the fundamental $l=s=0,2$ perturbations, respectively [70]. This can be compared to the numerical result [75, 10, 47] $M \omega=0.1105-0.1049 i, 0.3737-0.0890 i$. In the eikonal limit $(l \rightarrow \infty)$ the Pöschl-Teller approximation yields the correct solution: it predicts the behavior $3 \sqrt{3} M \omega= \pm(l+1 / 2)-i(n+1 / 2)$, in agreement with WKB-based calculations [58, 188, 189] (see also [190]).

The Pöschl-Teller approximation provides a solution which is more and more accurate for near-extremal SdS BHs, since the event horizon and the cosmological horizon coalesce in the extremal limit [162, 191, 192.

Normal modes of the anti-de Sitter spacetime A physically interesting analytical solution concerns the QNMs of pure AdS spacetime, which can be obtained by setting $r_{0}=0$ in the metric (3). In this case, QNMs are really normal modes of the spacetime, and have been computed for scalar field perturbations by Burgess and Lütken [193. They satisfy

$$
L \omega=2 n+d+l-1, \quad n=0,1,2, \ldots \quad s=0,
$$

where $l$ is related to the eigenvalue of spherical harmonics in $d$ dimensions by $A_{l m}=l(l+d-3)$ [153. Normal modes of electromagnetic perturbations in $d=4$ were shown to be the same as normal modes for gravitational perturbations (see Appendix in Ref. [194]); for general $d$, they can be computed from the potentials for wave 
propagation derived by Kodama and Ishibashi [104. Ref. [107] studies the normal modes of gravitational perturbations. Tensor gravitational perturbations obey the same equation as $s=0$ fields, and therefore their normal modes are

$$
L \omega=2 n+d+l-1, \quad n=0,1,2, \ldots \quad s=2 \text { tensor }- \text { type } .
$$

Vector-type gravitational perturbations have normal modes with the following characteristic frequencies

$$
L \omega=2 n+d+l-2, \quad n=0,1,2, \ldots \quad s=2 \text { vector }- \text { type } .
$$

Finally, scalar-type gravitational perturbations have a somewhat surprising behavior. For $d=4$ they are given by Eq. (47). For $d=5$ they have a continuous spectrum, and for $d>5$ one finds 107

$$
L \omega=2 n+d+l-3, \quad n=0,1,2, \ldots \quad s=2 \text { scalar }- \text { type } .
$$

Quasinormal modes of the de Sitter spacetime The dS spacetime is an extensively studied solution of the Einstein field equations, most of the early investigations being motivated by cosmological considerations. It satisfies Eq. (3) with $r_{0}=0$ and $f(r)=1-r^{2} / L^{2}$. Natário and Schiappa found that no QNM solutions are allowed in even-dimensional dS space [107]. For scalar fields and tensor-type gravitational perturbations in odd-dimensional dS backgrounds the QNM frequencies are purely imaginary, and given by

$$
L \omega=-i(2 n+d+l), \quad n=0,1,2, \ldots \text { tensor }- \text { type } .
$$

For the other types of gravitational perturbations Ref. 107] finds

$$
\begin{array}{ll}
L \omega=-i(2 n+d+l+1), & n=0,1,2, \ldots \text { vector }- \text { type }, \\
L \omega=-i(2 n+d+l+2), & n=0,1,2, \ldots \text { scalar }- \text { type } .
\end{array}
$$

Notice the striking similarity with the pure AdS results when one replaces $L \rightarrow i L$. Fields of other spins were considered in Refs. [195, 192.

Nearly-extreme $S d S$ and the Nariai spacetime The metric for $d$-dimensional Schwarzschild de-Sitter $\left(\mathrm{SdS}_{d}\right)$ BHs can be obtained from Eq. (3) by the replacement $L \rightarrow i L$, i.e., $f(r)=1-r^{2} / L^{2}-r_{0}^{d-3} / r^{d-3}$. The corresponding spacetime has two horizons: an event horizon at $r=r_{+}$and a cosmological horizon at $r=r_{c}$. It was observed in Ref. [196] that for $r_{c} / r_{+}-1 \ll 1$ perturbations of this spacetime satisfy a wave equation with a Pöschl-Teller potential. In particular, setting $k_{b} \equiv\left(r_{c}-r_{+}\right) / 2 r_{c}^{2}$, for near-extreme $\mathrm{SdS}_{d}$ one finds $M / r_{+}^{d-3} \sim 1 /(d-1), r_{+}^{2} / L^{2} \sim(d-3) /(d-1)$ [196, 197]. QNM frequencies for scalar field and tensor-like gravitational perturbations are then given by 196,197 .

$$
\frac{\omega}{k}=\sqrt{l(l+d-3)-\frac{1}{4}}-i\left(n+\frac{1}{2}\right), \quad \text { tensor - type, }
$$

where $k=(d-3)\left(r_{c}-r_{+}\right) /\left(2 r_{+}^{2}\right)$ is the surface gravity of the BH. For gravitational perturbations the result is

$$
\begin{aligned}
& \frac{\omega}{k}=\sqrt{(l-1)(l+d-2)-\frac{1}{4}}-i\left(n+\frac{1}{2}\right), \quad \text { vector }- \text { type }, \\
& \frac{\omega}{k}=\sqrt{(l-1)(l+d-2)-d+\frac{15}{4}}-i\left(n+\frac{1}{2}\right), \quad \text { scalar }- \text { type } .
\end{aligned}
$$


Through an appropriate limiting procedure [196, 198, the nearly-extreme $\mathrm{SdS}_{d}$ geometry can yield a spacetime with a different topology, the Nariai spacetime [196, 199, 200, 201], of the form

$$
d s^{2}=-\left(-r^{2} / L^{2}+1\right) d t^{2}+\left(-r^{2} / L^{2}+1\right)^{-1} d r^{2}+r^{2} d \Omega_{d-2}^{2} .
$$

This manifold has topology $d S_{2} \times S_{d-2}$ and two horizons (with the same surface gravity). The QNM frequencies are the same as for nearly-extreme $\mathrm{SdS}_{d}$, if $k$ is replaced by the surface gravity of each horizon [198].

The BTZ black hole Ichinose and Satoh 202] were the first to realize that the wave equation in the $(2+1)$-dimensional, asymptotically AdS BTZ BH 184 can be solved in terms of hypergeometric functions. An analytical solution for the QNMs of this $\mathrm{BH}$ was first found in Ref. 95. The non-rotating BTZ BH metric is given by 184

$$
d s^{2}=\left(r^{2} / L^{2}-M\right) d t^{2}-\left(r^{2} / L^{2}-M\right)^{-1} d r^{2}-r^{2} d \phi^{2},
$$

where $M$ is the $\mathrm{BH}$ mass and $r_{+}=M^{1 / 2} L$ is the horizon radius. In $2+1$ dimensions gravity is "trivial": the full curvature tensor is completely determined by the local matter distribution and the cosmological constant. In particular, in vacuum the curvature tensor $R_{\mu \nu \lambda \rho}=\Lambda\left(g_{\mu \lambda} g_{\nu \rho}-g_{\mu \rho} g_{\nu \lambda}\right)$ and $R=6 \Lambda$. Curvature effects produced by matter do not propagate through the spacetime. There are no dynamical degrees of freedom, and no gravitational waves 203, 204. Therefore, we will focus on scalar fields and assume an angular dependence of the form $e^{i m \phi}$. Scalar QNM frequencies are given by

$$
\omega L= \pm m-2 i(n+1) r_{+} / L,
$$

with $m$ the azimuthal number, and $n$ the overtone number 95. This result has been generalized by Birmingham, Sachs and Solodukhin [94] to the rotating BTZ BH. For general massive scalar perturbations with mass parameter $\mu$ they find

$$
\omega L= \pm m-i\left[2 n+\left(1+\sqrt{\mu^{2}+1}\right)\right]\left(r_{+}-r_{-}\right) / L .
$$

The BTZ background has provided a first quantitative test of the AdS/CFT correspondence: the QNM frequencies (57) match the poles of the retarded correlation function of the corresponding perturbations in the dual CFT [94]. Recently the QNMs of BTZ BHs were shown to be Breit-Wigner-type resonances generated by surface waves supported by the boundary at infinity, which acts as a photon sphere [205. This interpretation is highly reminiscent of work in asymptotically flat spacetimes, interpreting QNMs as null particles slowly leaking out of circular null geodesics (see Refs. [59, 73, 70, 206, 207, 208, 209] and Section 4.2 below).

An alternative to Einstein's gravity in three dimensions is the so-called "topologically massive gravity", obtained by adding a Chern-Simons term to the action 210, 211. Topologically massive gravity allows for dynamics, i.e. gravitational waves. The QNMs of BTZ BHs in this theory have recently been computed, providing yet another confirmation of the AdS/CFT correspondence [185, 186].

Massless topological black holes It is also possible to obtain exact solutions in a restricted set of higher-dimensional $\mathrm{BH}$ spacetimes. These asymptotically AdS solutions are known as topological BHs. The horizon is an Einstein space of positive, zero, or negative curvature [122, 123, 124, 125, 126, 127]. In the negative-curvature 
case there is a massless $\mathrm{BH}$ playing a role quite similar to the BTZ BH in three dimensions. Consider the exterior region of the massless topological BH 212 ]

$$
d s^{2}=-\left(r^{2} / L^{2}-1\right) d t^{2}+\left(r^{2} / L^{2}-1\right)^{-1} d r^{2}+r^{2} d \sigma^{2} .
$$

This is a manifold of negative constant curvature with an event horizon at $r=L$. Here $d \sigma^{2}$ stands for the line element of a $(d-2)$-dimensional surface $\Sigma_{d-2}$ of negative constant curvature.

The wave equation for a massive scalar field with non-minimal coupling can be solved by the ansatz $\Phi=\Psi(r) e^{-i \omega t} Y$, where $Y$ is a harmonic function of finite norm with eigenvalue $-Q=-(d-3)^{2} / 4-\xi^{2}$. The parameter $\xi$ is generically restricted, assuming only discrete values if $\Sigma_{d-2}$ is a closed surface. If the effective mass $m_{\text {eff }}^{2}=\mu^{2}-\gamma d(d-2) / 4 L^{2}$ (with $\mu$ the mass of the field and $\gamma$ the conformal coupling factor) satisfies the bound $m_{\text {eff }}^{2} L^{2} \geq-[(d-1) / 2]^{2}$, one set of QNM frequencies is given by 212 .

$$
\omega L= \pm \xi-i\left(2 n+1+\sqrt{(d-1)^{2} / 4+m_{\text {eff }}^{2} L^{2}}\right), \quad n=0,1,2 \ldots .
$$

If the mass and the coupling constant $\gamma$ satisfy the relations $\sqrt{(d-1)^{2} / 4+m_{\text {eff }}^{2} L^{2}}=$ $\frac{d-1}{2}-\frac{\gamma}{2} \frac{d-2}{d-1-\gamma(d-2)}$ and $-\left(\frac{d-1}{2}\right)^{2}<m_{\text {eff }}^{2} L^{2}<1-\left(\frac{d-1}{2}\right)^{2}$, there is another set of modes for which the QNM frequencies are given by 212 .

$$
\omega L= \pm \xi-i\left(2 n+1-\sqrt{(d-1)^{2} / 4+m_{\mathrm{eff}}^{2} L^{2}}\right) .
$$

This computation represents the first exact analytic determination of QNMs in four and higher dimensions. The generalization to other fields (and in particular to gravitational perturbations) can be found in Ref. 213.

\subsection{The WKB approximation}

Normal modes of vibration of an object usually have a simple interpretation in terms of waves traveling across or around the object. For example, the Earth's free modes of oscillation were highly excited and measured for the first time in the 1960 earthquake in Chile 214. These (roughly) one-hour long periodic oscillations correspond to waves traveling around the globe, and carry information about the Earth's interior. Just like the Earth's free modes of oscillation, BH QNMs can be thought of as waves traveling around the $\mathrm{BH}$ [58, 59, 70, 73, 206, 207, 208, 209]. More precisely, QNMs can be interpreted as waves trapped at the unstable circular null geodesic (also known as the light-ring) and slowly leaking out. The instability timescale of the geodesic is the decay timescale of the QNM, and the oscillation frequency $\omega \sim c / r_{\mathrm{LR}}$, with $c$ the speed of light and $r_{\mathrm{LR}}$ the light-ring radius [208].

This intuitive picture, first proposed by Goebel [59], is related to a more rigorous WKB approximation developed by Mashhoon 69] and by Schutz and Will [74] (see also [58, 70, 73, 206, 207, 208, 209]). Their derivation and results closely parallel the Bohr-Sommerfeld quantization rule from quantum mechanics. The procedure involves relating two WKB solutions across a "matching region" whose limits are the classical turning points, where $\omega^{2}=V(r)$. The technique works best when the classical turning points are close, i.e. when $\omega^{2} \sim V_{\max }$, where $V_{\max }$ is the peak of the potential. Under these assumptions we can expand in a Taylor series around the extremum of the potential $\bar{r}_{*}$ :

$$
Q \equiv \omega^{2}-V \sim Q_{0}+Q_{0}^{\prime \prime}\left(r_{*}-\bar{r}_{*}\right)^{2} / 2, \quad Q_{0}^{\prime \prime} \equiv d^{2} Q / d r_{*}^{2}
$$


In this region, the wave equation $\frac{d^{2} \Psi}{d r_{*}^{2}}+Q \Psi=0$ can be approximated by

$$
\frac{d^{2} \Psi}{d r_{*}^{2}}+\left[Q_{0}+\frac{1}{2} Q_{0}^{\prime \prime}\left(r_{*}-\bar{r}_{*}\right)^{2}\right] \Psi=0 .
$$

This equation has an exact solution in terms of parabolic cylinder functions [187, 215]:

$$
\Psi=A D_{\nu}(z)+B D_{-\nu-1}(i z), \quad z \equiv\left(2 Q_{0}^{\prime \prime}\right)^{\frac{1}{4}} e^{i \frac{\pi}{4}}\left(r_{*}-\bar{r}_{*}\right),
$$

with $\nu=-i Q_{0} / \sqrt{2 Q_{0}^{\prime \prime}}-1 / 2$. Using the asymptotic behavior of cylinder functions 187. 215] and demanding only outgoing waves at spatial infinity we get, near the horizon,

$$
\Psi \sim A e^{-i \pi \nu} z^{\nu} e^{-\frac{z^{2}}{2}}-i \sqrt{2 \pi} A[\Gamma(-\nu)]^{-1} e^{5 i \pi / 4} z^{-\nu-1} e^{\frac{z^{2}}{2}} .
$$

QNM boundary conditions imply that the outgoing term, proportional to $e^{z^{2} / 2}$, should be absent, so $1 / \Gamma(-\nu)=0$, or $\nu=n(=0,1,2, \ldots)$. As we anticipated, the leadingorder WKB approximation yields a "Bohr-Sommerfeld quantization rule" defining the QNM frequencies:

$$
Q_{0} / \sqrt{2 Q_{0}^{\prime \prime}}=i(n+1 / 2), \quad n=0,1,2, \ldots
$$
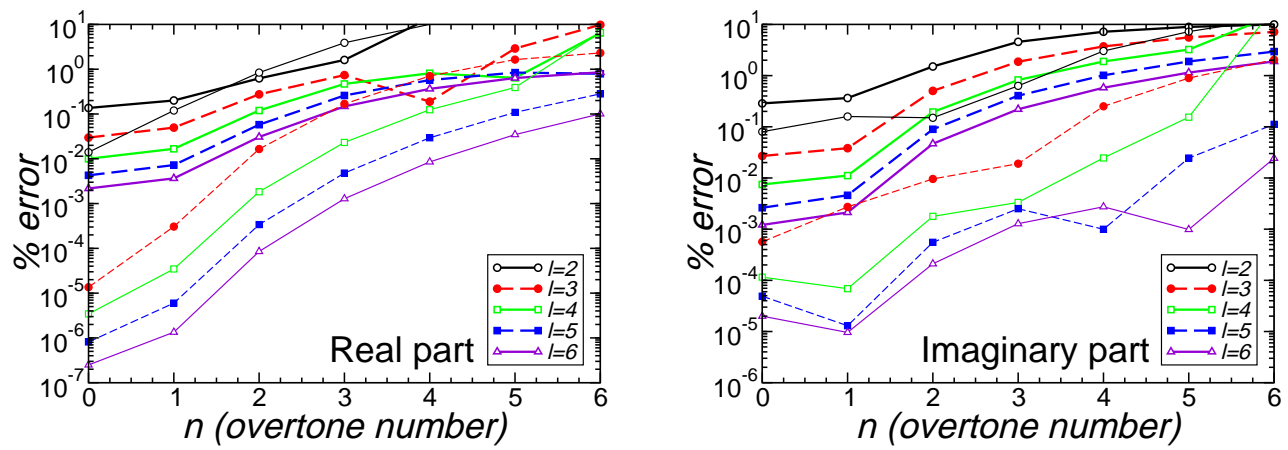

Figure 3. Percentage errors for the real (left) and imaginary part (right) of the QNM frequencies as predicted from WKB calculations. Thick lines: third-order WKB approximation; thin lines: sixth-order WKB approximation.

Higher-order corrections to Eq. [60) have been computed [58]. Iyer and Will [188, 189. carried out a third-order WKB expansion, and more recently Konoplya 216] pushed the expansion up to sixth order. There is no rigorous proof of convergence, but the results do improve for higher WKB orders. This is shown in Fig. 3, where we compare numerical results for the QNMs of Schwarzschild BHs from Leaver's continued fraction method (to be discussed in Section 5.1 below) against third- and sixth-order WKB predictions. The WKB approximation works best for low overtones, i.e. modes with a small imaginary part, and in the eikonal limit of large $l$ (which corresponds to large quality factors, or large $\left.\omega_{R} / \omega_{I}\right)$. The method assumes that the potential has a single extremum, which is the case for most (but not all) BH potentials: see Ref. 103 for interesting counterexamples.

Ref. 217] introduces a new, WKB-inspired asymptotic expansion of QNM frequencies and eigenfunctions in powers of the angular momentum parameter $l+1 / 2$. 
Their asymptotic expansion technique is easily iterated to high orders, and it seems to provide very accurate results in spherically-symmetric spacetimes. The asymptotic expansion also provides physical insight into the nature of QNMs, nicely connecting the geometrical understanding of QNMs as perturbations of unstable null geodesics with the singularity structure of the Green function.

\subsection{Monodromy technique for highly-damped modes}

A powerful variant of the WKB approximation, which is particularly useful in the highly-damped limit $\omega_{I} \rightarrow \infty$, is the so-called monodromy technique [101]. The basic idea is related to Stokes' phenomenon in the theory of asymptotic expansions (see e.g. 218 for an excellent introduction to the topic). As shown by Andersson and Howls [219], the monodromy technique is a simple variant of the phase-integral approach, whose application to $\mathrm{BH}$ physics dates back to the work by Fröman et al. 81.

Let us consider the wave equation (10) for the Schwarzschild geometry, but allowing $r$ and $r_{*}$ to be complex variables. In the complex- $r$ plane, solutions to Eq. (10) may be multivalued around the singular points $(r=0$ and $r=2 M)$. To deal with the singular points we introduce branch cuts emanating from $r=0$ and $r=2 M$. The relation $r_{*}(r)$ is also multi-valued: in the Schwarzschild geometry $r_{*}(r)=r+2 M \log (r / 2 M-1)$, and we choose the branch such that $\log (-1)=i \pi$. We can now define a variable $z \equiv r_{*} / 2 M-i \pi$ which tends to zero as $r \rightarrow 0$. The Stokes lines are defined as the lines for which $\operatorname{Re}\left(r_{*}\right)=0$ [218, and they are shown in Fig. 4 ] (near the singular point $r=0$, the Stokes lines form an angle $\pi / 4$ ). The idea now is
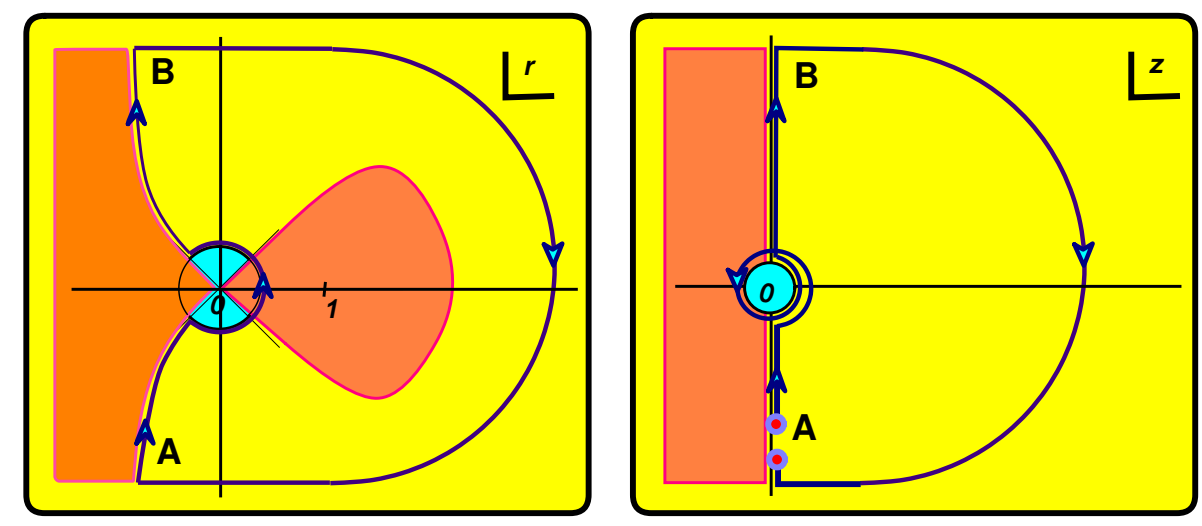

Figure 4. Left panel: contour for calculation of the QNM frequencies in the complex- $r$ plane. The different regions are separated by the associated Stokes lines. Not shown in the plot are branch cuts from $r=-\infty$ to the origin and from $r=1$ to point A. Right panel: integration contour in the complex- $z$ plane, with $z \equiv r_{*} / 2 M-i \pi$. For more details see Ref. 101.

to equate the monodromy as computed in two different ways. The first computation takes the contour in Fig. 4, starting and ending at point $\mathrm{A}$ and following the Stokes lines, joined at infinity by the large semi-circles shown in Fig. 4 . If we start from A with a plane wave $e^{i \omega z}$, we can extrapolate the behavior to near the singularity $z=0$. This is because for large imaginary $\omega$ the term $\omega^{2}$ is much larger than the other terms 
in the potential. Near the origin $(r=0)$ the solutions to the wave equation can be written as

$$
\Psi(z)=A_{+} \sqrt{4 \pi M \omega z} J_{\frac{s}{2}}(2 M \omega z)+A_{-} \sqrt{4 \pi M \omega z} J_{-\frac{s}{2}}(2 M \omega z),
$$

where $A_{ \pm}$are constants and $J_{s / 2}$ is a Bessel function. From the asymptotics of Bessel functions for large $\omega z$ one has

$$
\Psi(z) \sim e^{i 2 M \omega z}\left[A_{+} e^{-i \alpha_{+}}+A_{-} e^{-i \alpha_{-}}\right]+e^{-i 2 M \omega z}\left[A_{+} e^{i \alpha_{+}}+A_{-} e^{i \alpha_{-}}\right],
$$

with $\alpha_{ \pm}=\pi(1 \pm s) / 4$. The second term must vanish because of the boundary conditions. Continuing along the contour, we make a $3 / 2 \pi$ turn around $r=0$. In terms of the $z$ coordinate, this means a $3 \pi$ rotation. Using the representation for the Bessel functions

$$
J_{ \pm s / 2}(\eta)=\eta^{ \pm s / 2} \sum_{n=0}^{+\infty} \frac{(-1)^{n} \eta^{2 n}}{2^{2 n \pm s / 2} \Gamma( \pm s / 2+n+1) n !},
$$

one can see that $J_{ \pm s / 2}\left(e^{3 i \pi} 2 M \omega z\right)=e^{ \pm i 3 \pi s / 2} J_{ \pm s / 2}(2 M \omega z)$. Therefore

$$
\Psi \sim e^{i 2 M \omega z}\left[A_{+} e^{7 i \alpha_{+}}+A_{-} e^{7 i \alpha_{-}}\right]+e^{-i 2 M \omega z}\left[A_{+} e^{5 i \alpha_{+}}+A_{-} e^{5 i \alpha_{-}}\right] .
$$

The $e^{-i 2 M \omega z}$ term is exponentially small, since we are in the region where $\operatorname{Re}(z) \gg 0$ and $\omega_{I} \ll 0$. Using Eq. [66] we get for the coefficient of $e^{i \omega z}$ the monodromy

$$
\frac{A_{+} e^{7 i \alpha_{+}}+A_{-} e^{7 i \alpha_{-}}}{A_{+} e^{-i \alpha_{+}}+A_{-} e^{-i \alpha_{-}}} \text {. }
$$

Requiring that the second term in Eq. (66) vanishes, Eq. (69) yields $-(1+2 \cos (\pi s))$ for the monodromy. On the other hand, the only singularity inside the contour is at $r=1$, giving a factor $e^{-4 i(2 M \omega)}$ and leading to the condition

$$
8 \pi M \omega= \pm \log 3-i \pi(2 n+1)
$$

for the highly damped QNM frequencies of Schwarzschild BHs with $s=0,2(s=1$ is predicted to have a vanishing real part). This formula is in agreement with numerical studies by Nollert [220] for the Schwarzschild spacetime. The procedure has been generalized to several other backgrounds, including Reissner-Nordström (RN) 101, Kerr [221, 222, SAdS [223] and other BH spacetimes [107], always finding excellent agreement with numerical calculations [224, 225, 194.

The application of the monodromy method requires extreme care in identifying the appropriate Stokes lines and integration contours. Natário and Schiappa 107. point out some instances of inappropriate applications of the technique. A more rigorous mathematical treatment of these methods is highly desirable.

\subsection{Asymptotically anti-de Sitter black holes: a series solution}

With the exception of the monodromy technique, the methods discussed so far work for asymptotically flat or (with some minor modifications) dS spacetimes. For asymptotically AdS spacetimes, the perturbation equations exhibit regular singularities both at the horizon and at spatial infinity. Local solutions in the vicinity of the regular singular points are represented by convergent Frobenius series [226]. In many cases, the radius of convergence of the series is equal to or larger than the interval of interest. In this case, a simple numerical procedure (implemented e.g. by Horowitz and Hubeny in [92]) is possible. Consider, for example, a scalar field $\Psi$ 
in the $\mathrm{SAdS}_{4}$ spacetime, satisfying the wave equation (10). Defining $\Phi$ for a generic wavefunction as $\Phi=e^{i \omega r_{*}} \Psi$, we find

$$
f(r) \frac{d^{2} \Phi}{d r^{2}}+\left[f^{\prime}-2 i \omega\right] \frac{d \Phi}{d r}-\frac{V}{f} \Phi=0
$$

where $f^{\prime} \equiv d f / d r$. To find the frequencies $\omega$ that satisfy the boundary conditions we first note that Eq. (71) has only regular singularities in the range of interest. To deal with the point at infinity, we first change the independent variable $r$ to $x=1 / r$ and get

$$
\left(x-x_{+}\right) s(x) \frac{d^{2} \Phi}{d x^{2}}+t(x) \frac{d \Phi}{d x}+\frac{u(x)}{x-x_{+}} \Phi=0,
$$

where $x_{+}=1 / r_{+} \equiv h, s=-f x^{4} /\left(x-x_{+}\right), t=-\left[2 f x^{3}-f^{\prime} x^{2}-2 i \omega x^{2}\right], u=$ $V\left(x-x_{+}\right) / f$. Eq. (72) admits a local (near $x=h$ ) Frobenius solution of the form

$$
\Phi(x)=(x-h)^{\alpha} \sum_{n=0}^{\infty} a_{n}(x-h)^{n},
$$

where $a_{n}(\omega)$ is a function of the frequency. The radius of convergence of the series is limited by the distance to the next nearest singular point of the equation. The index $\alpha$ is determined by imposing the boundary condition at the horizon. Writing

$$
s(x)=\sum_{i=0}^{\infty} s_{i}(x-h)^{i}, t(x)=\sum_{i=0}^{\infty} t_{i}(x-h)^{i}, u(x)=\sum_{i=0}^{\infty} u_{i}(x-h)^{i},
$$

and substituting Eqs. (73) and (74) in Eq. (71) we get an indicial equation for $\alpha$ : $\alpha(\alpha-1) s_{0}+\alpha t_{0}+u_{0}=0$. We also have $s_{0}=2 h^{2} \kappa, t_{0}=2 h^{2}(\kappa-i \omega)$ and $u_{0}=0$, where the surface gravity is $\kappa=f^{\prime}\left(r_{+}\right) / 2$. Therefore, either $\alpha=0$ or $\alpha=i \omega / \kappa$. One can check that the second option corresponds to outgoing waves at the horizon, so we choose $\alpha=0$. Inserting the decomposition (73) with $\alpha=0$ in Eq. (71) and comparing powers of $(x-h)$ we get a recurrence relation for the $a_{n}$ 's:

$$
a_{n}=-\frac{1}{n(n-1) s_{0}+n t_{0}} \sum_{i=0}^{n-1}\left[i(i-1) s_{n-i}+i t_{n-i}+u_{n-i}\right] a_{i} .
$$

Since the differential equation is linear, $a_{0}$ is just an arbitrary normalization constant. Using the boundary condition $\Phi=0$ at infinity $(x=0)$ we finally obtain a simple relation determining the QNM frequencies:

$$
\sum_{n=0}^{\infty} a_{n}(-h)^{n}=0
$$

In practice one computes the $N$-th partial sum of the series (76) and finds the roots $\omega$ of the resulting polynomial expression, checking for convergence by comparison with (say) the roots obtained from the $N+1$-th partial sum. The method can be easily implemented: see e.g. the publicly available Mathematica notebook [4] that was used in 130 to compute low-lying modes of the $\mathrm{SAdS}_{4}$ geometry. This notebook can be easily modified to deal with other geometries.

Some mathematical aspects of the series solution method as applied to QNMs in asymptotically AdS backgrounds are discussed in Ref. 99]. 


\subsection{Asymptotically anti-de Sitter black holes: the resonance method}

The series solution described in the previous subsection converges very slowly for small SAdS BHs. Fortunately, in this regime there is a simple alternative: the resonance method 227. This method requires a numerical integration of the wave equation, but unlike the original numerical procedure by Chandrasekhar and Detweiler [62], one only needs to search for roots on the real- $\omega$ line.

It is well known that quasi-bound states manifest themselves as poles in the scattering matrix, and as Breit-Wigner resonances in the scattering amplitude. Chandrasekhar and Ferrari made use of the form of the scattering cross section near these resonances in their study of gravitational wave scattering by ultra-compact stars 228, 229. For quasi-bound, trapped modes of ultra-compact stars, the asymptotic wave amplitude at spatial infinity $\Psi \sim \alpha \cos \omega r+\beta \sin \omega r$ has a Breit-Wigner-type behavior close to the resonance:

$$
\alpha^{2}+\beta^{2} \approx \text { constant }\left[\left(\omega-\omega_{R}\right)^{2}+\omega_{I}^{2}\right],
$$

where $\omega_{I}^{-1}$ is the lifetime of the quasi-bound state and $\omega_{R}^{2}$ its characteristic "energy". The example of ultra-compact stars shows that the search for weakly damped QNMs corresponding to quasi-bound states $\left(\omega=\omega_{R}-i \omega_{I}\right.$ with $\left.\omega_{I} \ll \omega_{R}\right)$ is extremely simplified. One locates the resonances by looking for minima of $\alpha^{2}+\beta^{2}$ on the $\omega=\omega_{R}$ line, and the corresponding damping time $\omega_{I}$ can then be obtained by a fit to a parabola around the minimum [228, 229].

It was shown in [227] that these ideas can be used very successfully in $\mathrm{BH}$ spacetimes, if one integrates in from spatial infinity to the $\mathrm{BH}$ horizon (i.e., the appropriately re-defined quantities $\alpha^{2}+\beta^{2}$ are taken close to the horizon). The resonance method is extremely simple and accurate, especially for small SAdS BHs 227. (see also Section 6.1). It is presently unclear whether it can be applied successfully to study QNMs in other BH spacetimes.

\subsection{The continued fraction method}

Applications of the continued fractions to eigenvalue problems have an interesting history dating back to Jaffé's 1933 paper on the spectrum of the hydrogen molecule ion, or perhaps to even earlier times (some details and relevant references can be found in [99]).

The method was applied to gravitational problems by Leaver, leading to what is possibly the most successful algorithm to date to compute QNM frequencies 75, 76, 77. Leaver's approach is based on the observation that the Teukolsky equation is a special case of a general class of spheroidal wave equations that appear in the calculation of the electronic spectra of the hydrogen molecule ion [76]. These equations can be solved through a three-term recurrence relation, and the boundary conditions lead to a continued fraction equation characterizing the QNMs. Originally devised for the Schwarzschild and Kerr geometries [220, 230, 231, 225], the method has also been applied to RN BHs 232, Kerr-Newman BHs 233, higher-dimensional BHs [234, 235, 236, 237], SdS BHs [238, 239] and acoustic BHs [240] (see Section 10.4 below), among others. We illustrate the main ideas by considering the Kerr case. Start with the following series solution for the angular eigenfunctions defined in Eq. (25):

$$
{ }_{s} S_{l m}(u)=e^{a \omega u}(1+u)^{k_{-}}(1-u)^{k_{+}} \sum_{p=0}^{\infty} a_{p}(1+u)^{p},
$$


where $k_{ \pm} \equiv|m \pm s| / 2$. The expansion coefficients $a_{p}$ are obtained from the three-term recurrence relation

$$
\alpha_{0} a_{1}+\beta_{0} a_{0}=0, \quad \alpha_{p} a_{p+1}+\beta_{p} a_{p}+\gamma_{p} a_{p-1}=0, \quad p=1,2 \ldots
$$

where the constants $\alpha_{p}, \beta_{p}, \gamma_{p}$ are given in the original work [75]. Given a complex argument $\omega$, the separation constant ${ }_{s} A_{l m}$ can be obtained solving numerically the continued fraction $\beta_{0}-\frac{\alpha_{0} \gamma_{1}}{\beta_{1}-} \frac{\alpha_{1} \gamma_{2}}{\beta_{2}-} \frac{\alpha_{2} \gamma_{3}}{\beta_{3}-} \ldots=0$ or any of its inversions [75].

A solution $R_{r_{+}}$of the radial equation (25) should satisfy the appropriate boundary conditions:

$$
\begin{aligned}
& \lim _{r \rightarrow r_{+}} R_{r_{+}} \sim\left(r_{+}-r_{-}\right)^{-1-s+i \omega+i \sigma_{+}} e^{i \omega r_{+}}\left(r-r_{+}\right)^{-s-i \sigma_{+}}, \\
& \lim _{r \rightarrow \infty} R_{r_{+}} \sim A_{\text {out }}^{T}(\omega) r^{-1-2 s+i \omega} e^{i \omega r} .
\end{aligned}
$$

In these relations $\sigma_{+}=\left(\omega r_{+}-a m\right) / b$, and $b=\sqrt{1-4 a^{2}}$. A convenient series solution close to the horizon can be found by methods due to Jaffé (see [75]):

$$
R_{r_{+}}=e^{i \omega r}\left(r-r_{-}\right)^{-1-s+i \omega+i \sigma_{+}}\left(r-r_{+}\right)^{-s-i \sigma_{+}} \sum_{n=0}^{\infty} a_{n}^{r}\left(\frac{r-r_{+}}{r-r_{-}}\right)^{n}
$$

The coefficients $a_{n}^{r}$ can be obtained from a recurrence relation similar to Eq. (79). The continued fraction method is very powerful at computing overtones because the $n$-th overtone turns out to be the most stable numerical root of the $n$-th inversion of the radial continued fraction (which in principle should be completely equivalent to any other inversion) 75. This observation makes it (relatively) easy to numerically compute Kerr overtones up to $n \sim 50$, and Schwarzschild overtones up to $n \sim 100$; refinements of the technique to compute even higher overtones will be discussed in Section 5.3 below. Given the QNM frequencies and the corresponding angular eigenvalues ${ }_{s} A_{l m}$ it is a simple matter to compute $R_{r_{+}}$for moderate values of $r$ (the convergence of the expansion gets worse for large values of $r$ ). We provide an implementation of the method to compute the QNM frequencies, as well as the radial and angular wavefunctions, in a publicly available Mathematica notebook [47. On the same web page we also provide numerical data for the QNM frequencies of the first 8 Kerr gravitational modes $(n=0, \ldots, 7)$ with $2 \leq l \leq 7$, as computed by an independent Fortran code which will be made available upon request [241].

\section{The spectrum of asymptotically flat black holes}

In this section we briefly review the structure of the QNM spectrum of BHs belonging to the Kerr-Newman family. More details can be found in Ref. 241. We focus our discussion on gravitational perturbations, and refer to relevant works on other kinds of perturbations in the appropriate sub-sections.

\subsection{Schwarzschild}

For Schwarzschild BHs, scalar-type and vector-type gravitational perturbations can be shown to give rise to the same QNM spectrum. This remarkable result, due to Chandrasekhar and collaborators [22, 62, 141, is reviewed in Appendix A below.

The vector-type potential is simpler than the scalar-type potential, so it is customary to compute QNMs for vector-type perturbations. We computed QNM frequencies using the continued fraction technique (see Section 4.6) as improved by 

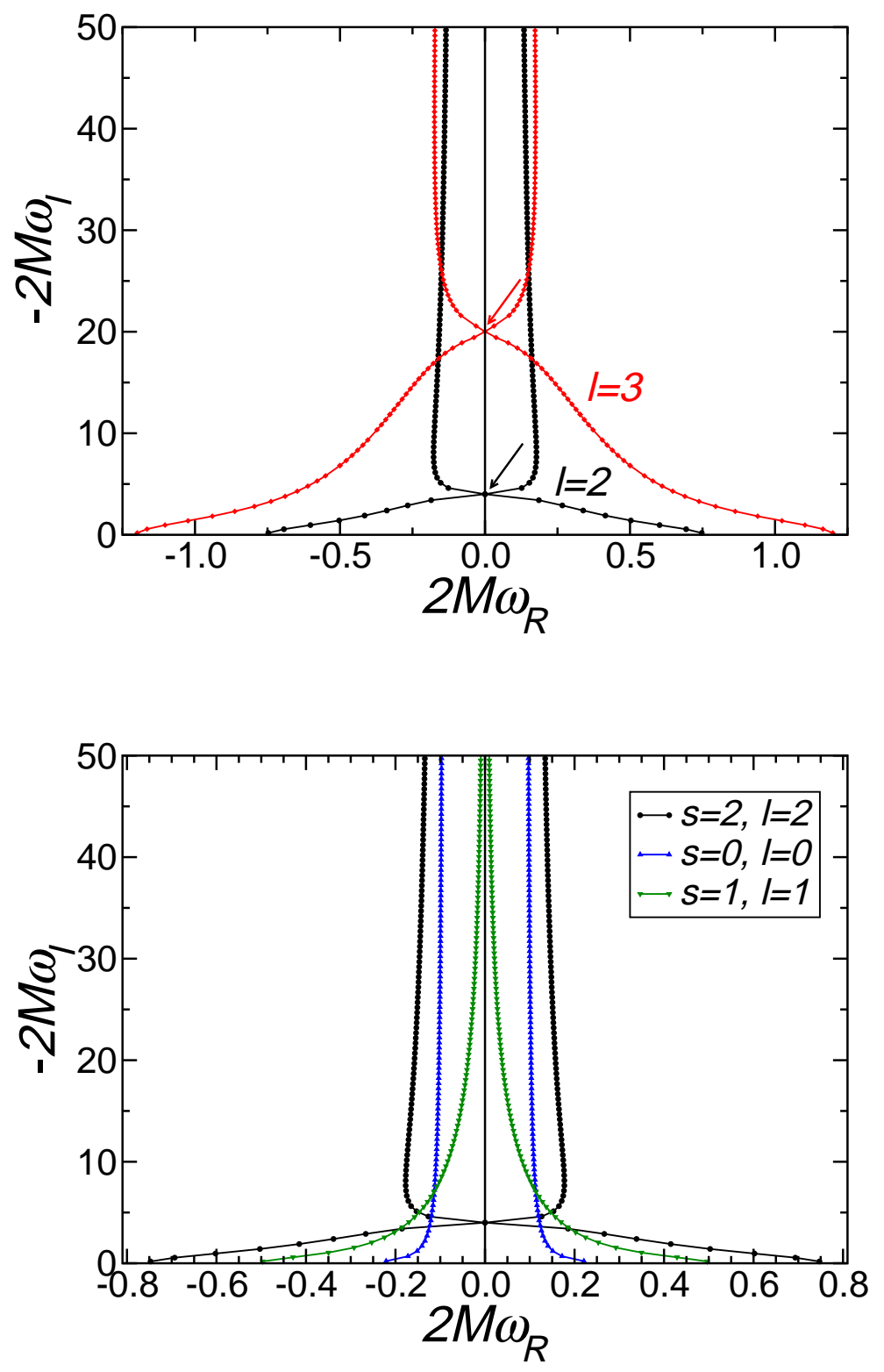

Figure 5. Top: QNM frequencies for gravitational perturbations with $l=2$ (black circles) and $l=3$ (red diamonds). In both cases we mark by an arrow the algebraically special mode, given analytically by Eq. (83); a more extensive discussion of this mode is given in Appendix A Notice that as the imaginary part of the frequency tends to infinity the real part tends to a finite, $l$-independent limit. Bottom: comparison of the $l=|s|$ QNM frequencies for scalar, electromagnetic and gravitational perturbations. 
Nollert [220]. Schwarzschild QNM frequencies with $l=2$ and $l=3$ are shown in Fig. 5. The data are also available online [47.

It is apparent from Fig. [5 that the gravitational QNMs of Schwarzschild BHs are naturally divided in two categories. A mode whose frequency is (almost) purely imaginary separates the lower QNM branch from the upper branch. This mode is very close to (and may actually coincide with) the so-called "algebraically special mode" [142, discussed in Appendix A. It is located at

$$
M \omega_{l} \approx \pm i(l-1) l(l+1)(l+2) / 12,
$$

and it can be taken as roughly marking the onset of the asymptotic high-damping regime. The algebraically special mode corresponds to an overtone index $n=9$ when $l=2$, to $n=41$ when $l=3$ and to even larger values of $n$ as $l$ increases. This means that the asymptotic high-damping regime is very hard to probe numerically as we approach the eikonal limit $(l \gg 1)$. The existence of an almost purely imaginary QNM frequency is unique to gravitational perturbations: for other known fields, the approach to the asymptotic regime is monotonic (see the bottom panel of Fig. [5 and Ref. 234]). For gravitational perturbations we will, somewhat arbitrarily, define the weakly damped (highly damped) regime as corresponding to imaginary parts smaller (larger) than the algebraically special frequency. For spins of other fields there is no such clear marker (see Fig. (5), but we will usually call "weakly damped" modes those with $n \lesssim 10$, and "highly damped" modes those with $n \gg 10$.

The weakly damped modes The weakly damped gravitational modes were computed numerically by Chandrasekhar and Detweiler 62, Leaver [75] and many others, and they are available online 47. Iyer 189. computed the first few modes for scalar, electromagnetic and gravitational perturbations using the WKB technique, and compared results against the more accurate continued fraction method. In geometrical units, the fundamental $l=|s|$ mode is $M \omega=0.1105-0.1049 i$ for $s=0$ and $M \omega=0.2483-0.0925 i$ for $s=1$. For ringdown detection from astrophysical BHs the most relevant QNM is, in most situations, the fundamental gravitational mode with $l=2$, with $M \omega=0.3737-0.0890 i$. This mode has oscillation frequency and damping time given by

$$
\begin{aligned}
& f=\omega_{R} / 2 \pi=1.207\left(\frac{10 M_{\odot}}{M}\right) \mathrm{kHz}, \\
& \tau=1 /\left|\omega_{I}\right|=0.5537\left(\frac{M}{10 M_{\odot}}\right) \mathrm{ms},
\end{aligned}
$$

where $M \sim 10 M_{\odot}$ is a typical value for a stellar-mass BH (see Section 9.3 below).

Weakly damped QNMs for Schwarzschild perturbations with half-integer spins have been computed in the WKB approximation by Cho and collaborators [147, 242.

The highly damped modes Using a variant of Leaver's method, Nollert carried out the first reliable numerical calculation of highly damped QNM frequencies for gravitational perturbations 220]. The real part of the QNM frequencies is well fitted, for large $n$, by a relation of the form $\omega_{R}=\omega_{\infty}+\lambda_{s, l} / \sqrt{n}$. The leading-order fitting coefficient $2 M \omega_{\infty} \simeq 0.08742$ is independent of $l$, and it has the same value for $s=2$ and $s=0$ [224. Nollert's numerical results have been confirmed by various analytical 
calculations. Motl [91] used Leaver's continued fraction conditions to show that in the limit $n \rightarrow \infty$ the following asymptotic expansion holds:

$$
\omega \sim T \ln 3-(2 n+1) \pi i T+\mathcal{O}\left(n^{-1 / 2}\right)
$$

where $T=(8 \pi M)^{-1}$ is the Hawking temperature. This conclusion was confirmed by complex-integration [101] and phase-integral techniques [219].

Corrections of order $\sim n^{-1 / 2}$ to Eq. (86) were first obtained by Neitzke [243] and Maassen van den Brink [244]. Ref. 245] developed a systematic perturbative approach to determine subdominant corrections as $n \rightarrow \infty$, using a solution of the Regge-Wheeler equation in terms of Bessel functions. Ignoring contributions of order $\mathcal{O}(1 / n)$, the result for $s=0$ and $s=2$ can be written as

$$
\frac{\omega}{T}=-(2 n+1) \pi i+\ln 3+\frac{1+i}{\sqrt{n+1 / 2}} \frac{3 l(l+1)-\left(s^{2}-1\right)}{18\left|s^{2}-1\right| \sqrt{2} \pi^{3 / 2}} \Gamma^{4}\left(\frac{1}{4}\right) .
$$

The subdominant coefficients are in agreement with fits of numerical results [220, 224, 234. For electromagnetic perturbations, analytic and numerical results suggest that the real part $\omega_{R} \rightarrow 0$ in the asymptotic limit [101, 234, which is also apparent from Fig. 5. The above calculation has been generalized to massless fermionic perturbations. By solving the Teukolsky equation in terms of confluent hypergeometric functions, Refs. 246, 247] confirmed the known results for integer spins, and found in addition that the result for $s=1 / 2$ and $s=5 / 2$ can be written as:

$$
\frac{\omega}{T}=-(2 n+1) \pi i+\frac{2(1+i)}{\left(s^{2}+23 / 4\right) \sqrt{n}}\left(3 l(l+1)+1-s^{2}\right) \Gamma^{2}\left(\frac{1}{4}\right) .
$$

For $s=3 / 2$ there is no correction at order $\mathcal{O}\left(n^{-1 / 2}\right)$. Numerical studies on $s=1 / 2$ fields confirm that $\omega_{R} \rightarrow 0$ and the spacing $\delta \omega_{I} \rightarrow 2 \pi T=1 / 4 M$ as $n \rightarrow \infty$ [248.

In summary, numerical and analytical results for Schwarzschild BHs are in perfect agreement. As $\left|\omega_{I}\right| \rightarrow \infty, \omega_{R} \rightarrow T \ln 3$ for scalar and gravitational oscillation frequencies, and $\omega_{R} \rightarrow 0$ for perturbations of other spins. The spacing of the imaginary parts for large $n$ is always given by $2 \pi T$. By considering the scattering amplitude in the Born approximation, Padmanabhan showed that this universality in the spacing arises from the exponential redshift of the wave modes close to the horizon [249].

The eikonal limit By their own nature, WKB methods become increasingly accurate for large $l$, and they can be used to compute the QNM frequencies analytically when $l \gg 1$. Up to order $\mathcal{O}\left(l^{-2}\right)$ the result is [58, 188, 189, 190]

$$
\begin{aligned}
M \omega & =\frac{1}{3 \sqrt{3}}\left[\left(l+\frac{1}{2}\right)-\frac{1}{3}\left(\frac{5\left(n+\frac{1}{2}\right)^{2}}{12}+\frac{115}{144}-1+s^{2}\right)\left(\frac{1}{l}-\frac{1}{2 l^{2}}\right)\right] \\
& -i \frac{\left(n+\frac{1}{2}\right)}{3 \sqrt{3}}\left[1+\frac{1}{9}\left(\frac{235\left(n+\frac{1}{2}\right)^{2}}{432}-\frac{1415}{1728}+1-s^{2}\right) \frac{1}{l^{2}}\right] .
\end{aligned}
$$

The convergence of the series gets worse with increasing $n$ (cf. also Fig. 3). In the eikonal limit the asymptotic behavior of the potential is not important, so it should not be surprising that a Pöschl-Teller approximation of the Schwarzschild potential, discussed in Section 4.1, yields the correct result (to leading order) for large $l$. The eikonal regime of Schwarzschild BHs is related to the properties of unstable circular null geodesics [58, 59, 70, 73, 206, 207, 208, 209]: the leading order of Eq. 89 can be written as $\omega=\Omega_{c} l-i(n+1 / 2) \lambda$, where $\Omega_{c}$ is the orbital frequency and $1 / \lambda$ is the instability timescale of the unstable circular null geodesic. Such a connection can be 
generalized to any asymptotically flat, static spacetime in four and higher dimensions 208 .

\subsection{Reissner-Nordström}

With a few exceptions, BH charge is usually considered astrophysically negligible. Despite this, the RN metric is of more than academic interest: for example, charged naked singularities have been proposed as classical models for elementary particles (see 233 and references therein). Handling scalar fields in the background of a charged $\mathrm{BH}$ requires only a straightforward generalization of the uncharged case, resulting in a wave equation of the form (10). Electromagnetic and gravitational perturbations are more technically challenging, since they are coupled through the Einstein-Maxwell equations. It is still possible to reduce the problem to the study of two wave equations of the general form (10]) for two wavefunctions $Z_{1}^{-}, Z_{2}^{-}$[22]:

$$
\begin{aligned}
& \left(\frac{d^{2}}{d r_{*}^{2}}+\omega^{2}\right) Z_{i}^{(-)}=V_{i}^{(-)} Z_{i}^{(-)}, \\
& V_{i}^{(-)}=\frac{\left(r^{2}-2 M r+Q^{2}\right)}{r^{5}}\left[l(l+1) r-q_{j}\left(1+\frac{q_{i}}{(l-1)(l+2) r}\right)\right] .
\end{aligned}
$$

Here $q_{1}+q_{2}=6 M,-q_{1} q_{2}=4 Q^{2}(l-1)(l+2)$ and $i, j=1,2(i \neq j)$. In the limit when the charge $Q / M$ goes to zero, $Z_{1}^{-}, Z_{2}^{-}$describe pure electromagnetic and pure vectortype gravitational perturbations of a Schwarzschild $\mathrm{BH}$, respectively. In the limit of maximally charged $\mathrm{BHs}(Q / M=1)$ the wave equations have a different singularity structure, and deserve a special treatment [250].

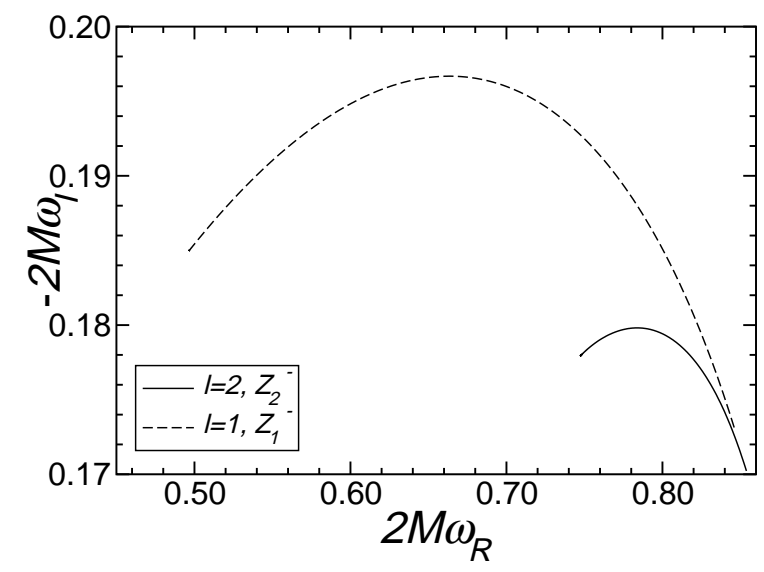

Figure 6. Trajectory the complex-frequency plane described by the fundamental QNM as the charge $Q / M$ is increased. The solid line corresponds to $l=2$ and $Z_{2}^{-}$(which reduces to vector-type perturbations as $Q / M \rightarrow 0$ ); the dashed line corresponds to $l=1$ and $Z_{1}^{-}$(purely electromagnetic perturbations in the limit $Q / M \rightarrow 0)$. The two modes coalesce in the extremal limit $(Q / M \rightarrow 1)$.

The weakly damped modes The behavior of the weakly damped modes of gravitational/electromagnetic perturbations is exemplified in Fig. 6. The solid line is 
the trajectory described in the complex-frequency plane by the fundamental QNM with $l=2$ corresponding to $Z_{2}^{-}$(perturbations that reduce to the vector-type gravitational Schwarzschild case as $Q / M \rightarrow 0)$. The dashed line is the trajectory of the fundamental QNM with $l=1$ corresponding to $Z_{1}^{-}$(which reduces to purely electromagnetic perturbations as $Q / M \rightarrow 0)$. The Schwarzschild limit corresponds to the bottom left of each curve, and the trajectories are described clockwise as $Q$ increases. The real part of the frequency grows monotonically with $Q$, and the imaginary part shows an extremum. A striking feature is that modes of $Z_{2}^{-}$with angular index $l$ coalesce with modes of $Z_{1}^{-}$with index $(l-1)$ in the extremal limit. We will elaborate on the significance of this result later in this section. In general there are two algebraically special frequencies for RN BHs [142], located at

$$
\omega_{1,2}= \pm \frac{i}{2} \frac{(l-1) l(l+1)(l+2)}{3 M \mp \sqrt{9 M^{2}+16 Q^{2}(l-1)(l+2)}} .
$$

Using the numerical procedure described by Chandrasekhar and Detweiler 62, Gunter computed the lowest-lying QNMs of electromagnetic/gravitational perturbations 251. Extensive comparisons of Gunter's results against WKB predictions were done by Kokkotas and Schutz 252. The continued-fraction method can be generalized to charged BHs with relatively minor modifications [232, and it was used to compute numerically the weakly and highly damped QNMs of gravitational/electromagnetic perturbations [232, 253, 250, 254, 241, 224. Weakly damped modes of massive charged fields were computed by WKB techniques in Ref. [255]. Dirac QNMs in the Kerr-Newman metric (which includes RN as a special case) have been computed by continued fractions in Ref. 256. For generalizations to higher-dimensional charged BHs, see 257] and references therein.

The highly damped modes Let us focus our attention on the high-damping regime for $Z_{2}^{-}$; results for $Z_{1}^{-}$are similar. For a more detailed discussion of highly-damped modes, see [224, 241]. The modes' behavior is better understood by looking separately at the
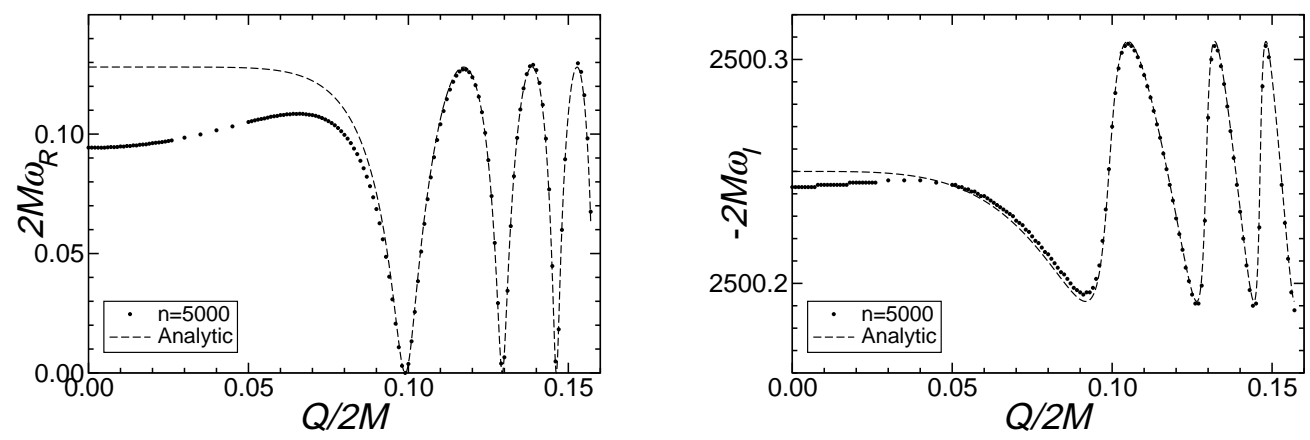

Figure 7. Numerical and analytical predictions for the real and imaginary part of the RN QNM frequencies as a function of charge for $n=5 \times 10^{3}$.

real and imaginary parts of their frequencies as functions of charge. Numerical results are shown in Fig. 7. Frequencies and damping times oscillate as a function of charge 
whenever $Q$ is larger than some $Q_{\text {crit }}$, and $Q_{\text {crit }} / M$ decreases with overtone number. For moderate-to-large values of $Q / M$, numerical data are in excellent agreement with the analytical prediction by Motl and Neitzke [101, 219, 107], valid for scalar and electromagnetic-gravitational perturbations of a $\mathrm{RN} \mathrm{BH}$ in the large-damping limit:

$$
e^{\omega / T}+2+3 e^{\left(Q^{4} \omega\right) /\left(r_{+}^{4} T\right)}=0, \quad T=\sqrt{M^{2}-Q^{2}} /\left[2 \pi r_{+}^{2}\right],
$$

where $r_{+}=M+\sqrt{M^{2}-Q^{2}}$ is the radius and $T$ is the Hawking temperature of the outer horizon. The complex solutions of Eq. (92) exactly overlap with the oscillations observed in Fig. 7 for large enough values of $Q / M$. This agreement gives support to the asymptotic formula (92), while confirming that the numerics are still accurate for large values of $n$ and $Q / M$ 243. However, Eq. (92) presents us with some striking puzzles. The predicted asymptotic RN QNM frequencies do not reduce to the expected Schwarzschild limit of Eq. (86): one finds instead $\omega_{R} \rightarrow T \ln 5$ as $Q / M \rightarrow 0$ [101, 243]. Eq. (92) should also be taken with care in the extremal limit $(Q / M \rightarrow 1)$ : in this case the inner and outer horizons coalesce, the topology of the singularities in the differential equation changes, and the problem requires a separate analysis [107]. Such an analysis shows that the asymptotic oscillation frequency for extremal RN BHs is not given by the limit of Eq. (92) as $Q / M \rightarrow 1$. Instead, the modes are purely damped $\left(\omega_{R} \rightarrow 0\right)$. An interesting classification of the solutions of Eq. (92) can be found in Fig. 3 and Section 4.4 of 219]. Besides "spiralling" solutions, the equation also admits periodic solutions when $\sqrt{1-Q^{2} / M^{2}}$ is a rational number, and even pure imaginary solutions that may not be QNMs at all. A survey of highly-damped QNM spectra of charged BHs can be found in Ref. 107. For a complete account of the asymptotic QNM spectrum of several perturbing fields in the RN metric, see Ref. [258].

The eikonal limit The eikonal regime $(l \gg 1)$ is well described by a WKB analysis. The lowest-order WKB approximation yields

$$
\omega_{R} \sim(l+1 / 2) \Omega_{c}, \quad \omega_{I} \sim-1 / 2 \Omega_{c} \sqrt{3 M / r_{0}-4 Q^{2} / r_{0}^{2}},
$$

where $r_{c}$ and $\Omega_{c}$ are the radius and frequency of the unstable circular null geodesic, $r_{c}=\left(3 M+\sqrt{9 M^{2}-8 Q^{2}}\right) / 2$ and $\Omega_{c}=\sqrt{M / r_{0}^{3}-Q^{2} / r_{0}^{4}}$ [252, 254]. QNMs in this regime can also be interpreted, from a geometrical-optics point of view, as waves trapped at the unstable circular null geodesics [73, 208].

Extremal Reissner-Nordström A direct application of Leaver's method fails in the extremal limit. In this limit the two horizons coalesce, and the radial wave equation has irregular singular points at the horizon and at infinity. Onozawa et al. 250. managed to reduce the problem to a five-term recurrence relation. As anticipated from Fig. 6] the QNM spectrum for extremal RN BHs is characterized by an isospectrality between electromagnetic and gravitational perturbations: modes of $Z_{2}^{-}$with angular index $l$ coalesce with modes of $Z_{1}^{-}$with index $(l-1)$ in the extremal limit. This has been shown to be a manifestation of supersymmetry [259, 260, 261.

The resulting QNM spectrum [241, 224, 250] looks qualitatively similar to the Schwarzschild spectrum of Fig. 5, The real part first decreases, approaching the pure-imaginary axis as the overtone index grows. A QNM seems to be located at $\omega=(0,-3.0479)$, while Chandrasekhar's formula (91) predicts a mode at $\omega=(0,-3)$. The numerical method used so far becomes unreliable for $\left|\omega_{I}\right| \gtrsim 10$, and better techniques will be needed to verify analytical predictions [101, 107. 


\section{3. $K e r r$}

The Kerr QNM spectrum has a rich and complex structure [75, 230, 231, 241, 262. The most relevant feature of the spectrum is that rotation acts very much like an external magnetic field on the energy levels of an atom, causing a Zeeman splitting of QNM frequencies. The determination of the QNM frequencies is tangled to the solution of an angular equation, the spin-weighted spheroidal harmonic equation (see Sections 2.3 and 4.6). In the general case $a \omega \neq 0$ there are no known closed-form solutions for the separation constant $A_{l m}$ or for the spheroidal harmonics. However the spheroidal harmonics satisfy various symmetry properties [153, 75], namely:

(i) Eigenvalues for negative and positive $m$ are related: ${ }_{s} A_{l m}={ }_{s} A_{l-m}^{*}$.

(ii) Eigenvalues for negative and positive $s$ are related: ${ }_{-s} A_{l m}={ }_{s} A_{l m}+2 s$.

(iii) If $\omega$ and ${ }_{-s} A_{l m}$ correspond to a solution for given $(s, l, m)$, another solution can be obtained by the following replacements: $m \rightarrow-m, \omega \rightarrow-\omega^{*},{ }_{-s} A_{l m} \rightarrow{ }_{-s} A_{l-m}^{*}$. These symmetries are usually a source of some confusion, so we give an explicit numerical example. For $a=0.6 M$, the methods described in Section 4.6 yield the following eigenvalues for $s=-2$ :

$$
\begin{aligned}
& M \omega_{22}=0.49404-0.08376 i, \quad M \omega_{2-2}=-0.49404-0.08376 i, \\
& M \omega_{22}=-0.31678-0.08889 i, M \omega_{2-2}=0.31678-0.08889 i .
\end{aligned}
$$
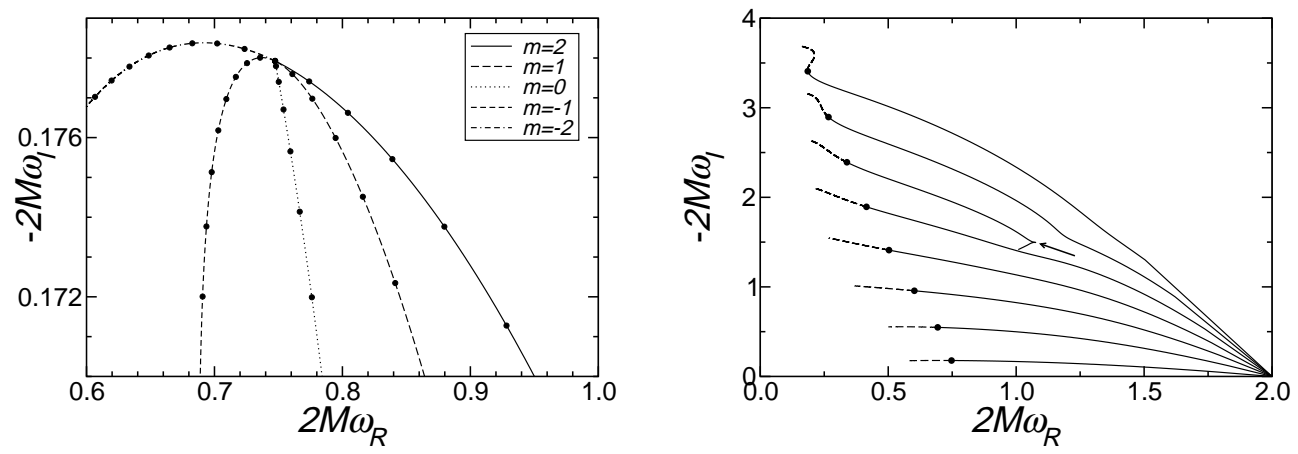

Figure 8. Left: "Zeeman-like" splitting of the fundamental gravitational mode with $l=2$. We mark by dots the points corresponding to $a / M=$ $0.0,0.1,0.2, \ldots 1.0$. Right: trajectory of the first eight Kerr QNM frequencies with $m=2$ (solid lines) and $m=-2$ (dashed lines). Filled circles mark the corresponding mode in the Schwarzschild limit. An arrow indicates the small loop described by the "exceptional" QNM with $n=6$, that does not tend to the critical frequency for superradiance (see also Figs. 3-4 in 230]). The data used to produce this figure (and more) are available online [4].

We illustrate the splitting of the fundamental gravitational QNM with $l=2$ in the left panel of Figure 8 . Even though QNMs have both positive and negative frequencies, it is customary to plot only the positive-frequency part of the spectrum [75]: in view of the symmetry properties listed above, this yields all the necessary information. As the rotation parameter $a / M$ increases, the branches with $m=2$ and $m=-2$ move in opposite directions, being tangent to the the branches with $m=1$ and $m=-1$ in the limit $a / M \rightarrow 0$. For low overtone numbers and small values 
of $a / M$ the rotation-induced splitting of the modes is roughly proportional to $m$, as physical intuition would suggest.

The weakly damped modes of Kerr black holes In the right panel of Figure 8 we show the first eight gravitational QNM frequencies with $m=2$ (solid lines) and $m=-2$ (dashed lines). A general feature is that almost all modes with $m>0$ cluster at the critical frequency for superradiance, $2 M \omega=m$, as $a / M \rightarrow 1$. This fact was first observed by Detweiler 262, and a thorough examination of the extremal limit can be found in Refs. 263, 264, 265. The mode with $n=6$ (marked by an arrow) shows a peculiar deviation from the general trend, illustrating the fact that some positive- $m$ modes do not tend to this critical frequency in the extremal limit.
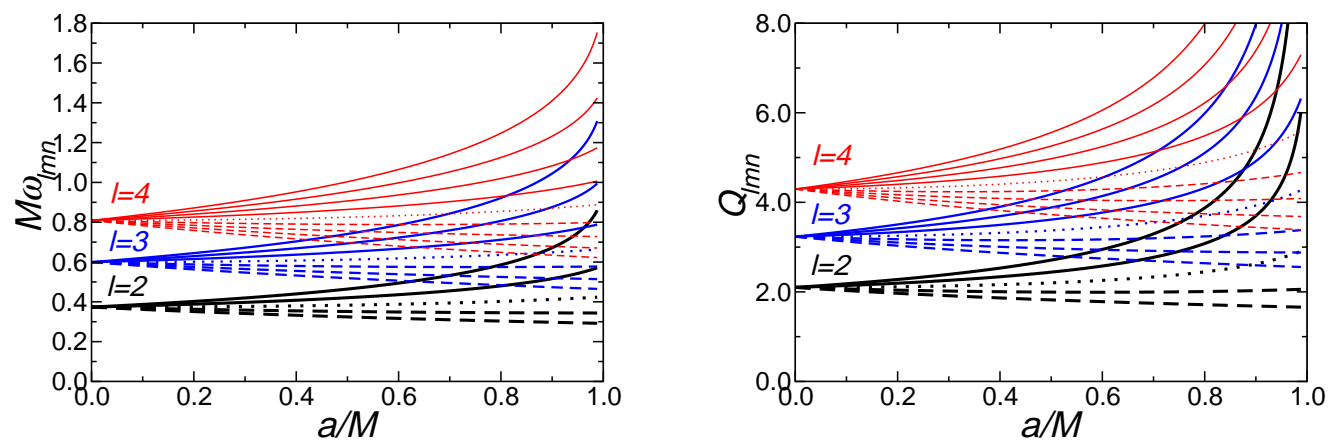

Figure 9. Frequencies and quality factors for the fundamental modes with $l=2,3,4$ and different values of $m$. Solid lines refer to $m=l, \ldots, l$ (from top to bottom), the dotted line to $\mathrm{m}=0$, and dashed lines refer to $m=-1, . .,-l$ (from top to bottom). Quality factors for the higher overtones are lower than the ones we display here.

For gravitational wave detection we are mostly interested in the frequency and quality factor of the dominant modes, which determine whether the mode lies in the sensitive frequency band of a given detector and the number of observable cycles. Figure 9 shows these quantities for QNMs with $l<5$. Improving on previous results [9, 266], Ref. [10] presented accurate fits for the first three overtones with $l=2,3,4$ and all values of $m$, matching the numerical results to within $5 \%$ or better over a range of $a / M \in[0,0.99]$ (see Tables VIII-X in Ref. [10] and the numerical data available online [47]). For instance, defining $\hat{b} \equiv 1-a / M$, the frequency $\omega_{l m}=\omega_{R}$ and quality factor $Q_{l m} \equiv \omega_{R} /\left(2 \omega_{I}\right)$ of the fundamental $l=m=2$ and $l=2, m=0$ modes are

$$
\begin{array}{ll}
M \omega_{22} \simeq 1.5251-1.1568 \hat{b}^{0.1292}, & Q_{22} \simeq 0.700+1.4187 \hat{b}^{0.4990}, \\
M \omega_{20} \simeq 0.4437-0.0739 \hat{b}^{0.3350}, & Q_{20} \simeq 4.000-1.9550 \hat{b}^{0.1420}
\end{array}
$$

The highly damped modes The intermediate- and large-damping regime of the QNM spectrum of Kerr BHs is even more puzzling than the RN spectrum. The main technical difficulty in pushing the calculation to higher damping is that Leaver's approach requires the simultaneous solution of the radial and angular continued 
fraction conditions. For mode order $n \gtrsim 50$ the method becomes increasingly unreliable. A way around this "coupling problem" is to study the asymptotic behavior of the angular equation as $|a \omega| \simeq\left|a \omega_{I}\right| \rightarrow \infty$ [225, 153. The leading-order behavior of the separation constant ${ }_{s} A_{l m}(a \omega)$ when $\omega \simeq i \omega_{I}$ and $|a \omega| \rightarrow \infty$ is

$$
{ }_{s} A_{l m}=i(2 L+1) a \omega+\mathcal{O}(1),|a \omega| \rightarrow \infty,
$$

with $L=l-|m|$ for $|m| \geq|s|$ and $L=l-|s|$ otherwise [225, 153]. By replacing this analytic expansion in the radial continued fraction one effectively decouples the angular and radial continued fractions, and the calculation of QNM frequencies with $n \gg 50$ can be performed by solving only the radial continued fraction. It turns out that in the highly damped limit $\omega_{R}$ is independent of $(s, l)$ and proportional to $m$ :

$$
2 M \omega_{R}=m \varpi(a / M) \text {. }
$$
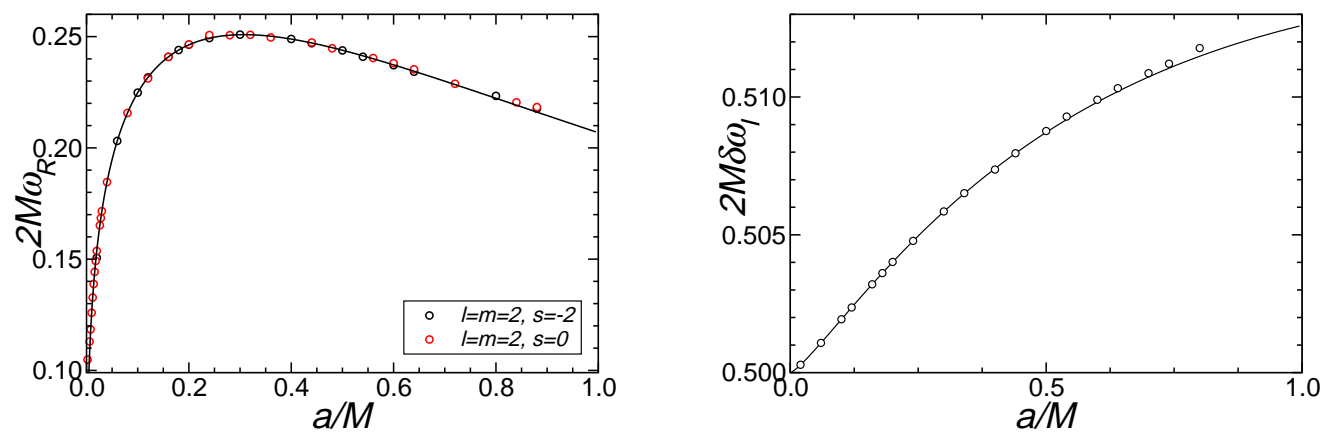

Figure 10. Left: asymptotic real part $2 M \omega_{R}=2 \varpi(a / M)$ of the $l=m=2$ gravitational and scalar QNM frequencies extrapolated from numerical data. Points refer to numerical results, the line is the analytical prediction. Results are independent of $l, s$ and the sign of $m$. Right: same for imaginary part.

Numerical results for $\varpi(a / M)$ are shown in Fig. 10, These numerical results have been confirmed by recent analytical calculations in an impressive tour de force 267, 268, 221, 222. The final result can be implicitly expressed as a contour integral, which in turn can be expressed as a sum of elliptic integrals [268, 222. The relevant equations are summarized in Appendix B, and compared against our own numerics in Fig. 10. The agreement is remarkable, given how involved the numerical and analytical calculations are. In the Schwarzschild limit $(a / M \rightarrow 0)$ it can be shown that $\varpi(a) \simeq 0.30634(a / M)^{1 / 3}$ and $2 M \delta \omega_{I} \simeq 1 / 2$. A good fit to the analytical predictions for the real part (accurate to within $0.8 \%$ in the entire range) is

$$
\varpi=0.307(a / M)^{1 / 3}-0.308 a / M+0.156(a / M)^{2}-0.052(a / M)^{3} .
$$

In the extremal limit $(a / M \rightarrow 1)$ the analytical results imply $\varpi \simeq 0.10341,2 M \delta \omega_{I} \simeq$ 0.51260 . For any $a / M$, the imaginary part $\omega_{I}$ grows without bound. The spacing between consecutive modes $\delta \omega_{I}$ is not simply given by $2 \pi T$, but it is a monotonically increasing function of $a / M$. A power fit in $a / M$ of the numerical results yields [225]:

$$
2 M \delta \omega_{I}=1 / 2+0.0219 a / M-0.0089(a / M)^{2} .
$$


The agreement between the analytical predictions for the mode spacing and the fit of Eq. (101) is better than $0.1 \%$ in the entire range of $a / M$. A generalization of these asymptotic results to higher-dimensional rotating BHs can be found in Ref. [222.

The eikonal limit The eikonal limit of Kerr QNMs is not yet fully understood. Partial results concern $l= \pm m$ modes, for which [73, 10, 208]

$$
\omega= \pm m \Omega_{c}-(n+1 / 2)\left|\Omega_{c}\right| \sqrt{3 M / r_{c}} \delta^{-1}, \quad(l=|m| \rightarrow \infty),
$$

where $r_{c}, \Omega_{c}$ are counter-rotating or co-rotating radius and orbital frequencies at the unstable circular null geodesics, and $2 \delta=r_{c}\left(r_{c}-M\right) /\left(r_{c}^{2}-2 M r_{c}+a^{2}\right)$. Again, this result can be expressed as $\omega \sim l \Omega_{c}-i(n+1 / 2) / \tau$, with $\tau$ the typical instability timescale of the unstable circular null geodesic [73, 10, 208. To our knowledge, a simple geometrical optics interpretation is still lacking for modes with $l \neq|m|$.

\subsection{Kerr-Newman}

General BH solutions of the Einstein-Maxwell system are described by the KerrNewman metric. For this metric the Klein-Gordon equation and the Dirac equation are still separable 269, so QNMs can be computed using the same general methods that apply to Kerr BHs 256]. The scalar spectrum was analyzed by Berti and Kokkotas [233, who showed in particular that the eikonal limit can still be understood in terms of unstable circular null geodesics.

Unfortunately, studies of the interplay of electromagnetic and gravitational fields in the Kerr-Newman metric are plagued by a major technical difficulty: to date, all attempts to decouple the electromagnetic and gravitational perturbations have failed (see e.g. Section 111 of Ref. [22]). Approximations to gravitational/electromagnetic perturbations of the Kerr-Newman geometry either keep the geometry fixed and perturb the electric field, or (more interestingly) keep the electric field fixed and perturb the geometry. This approach should be appropriate for values of the charge $Q$ at most as large as the perturbations of the spacetime metric. QNMs for gravitational and electromagnetic perturbations in this approximation scheme were computed in Refs. [270, 233], but a solution of the general problem is highly desirable, and it could shed light on many open problems in $\mathrm{BH}$ physics.

\subsection{Higher dimensional Schwarzschild-Tangherlini black holes}

Here we briefly discuss the QNM spectrum of the higher-dimensional analogs of the Schwarzschild solution, known as Schwarzschild-Tangherlini BHs. Electromagnetic perturbations of these BHs were considered by Crispino, Higuchi and Matsas [134. The elegant work by Kodama and Ishibashi [102, 103, 104] laid the foundations for the analysis of higher-dimensional RN BHs.

Weakly damped modes The lowest lying QNMs of a $d$-dimensional SchwarzschildTangherlini BH were computed in a WKB approximation in Refs. [216, 271, 272, 204. Leaver's method can be generalized to these higher-dimensional BHs [234, 235, although the number of terms in the recurrence relation rapidly grows with $d$. For instance, in $d=5$ the recurrence relation for vector-gravitational and tensorgravitational perturbations has four terms, while for scalar-type gravitational QNM frequencies it has eight terms [235]. A naive application of Leaver's method breaks 
down for $d>9$. For large $d$ more and more singularities, spaced uniformly on the circle $|r|=r_{h}$ (where $r_{h}$ is the horizon radius) approach the horizon at $r=r_{h}$. A solution satisfying the outgoing wave boundary condition at the horizon must be continued to some mid point, and only then can the continued fraction condition be applied 273. Alternative calculations of weakly damped modes for scalar and gravitational perturbations in $d$ dimensions make use of time evolutions [274].

Highly-damped modes In the large-damping limit, the leading-order result of the monodromy calculation, Eq. (86), generalizes to the $d$-dimensional SchwarzschildTangherlini metric. This was first suggested in Ref. [101], and then it was explicitly shown by Birmingham 275] (see also [276]). The perturbative technique of Ref. 245] has been extended to bosonic fields in higher-dimensional Schwarzschild-Tangherlini BHs, with the result 234

$$
\omega=T \ln (1+2 \cos \pi j)+(2 n+1) \pi i T+k_{d} \omega_{I}^{-(d-3) /(d-2)} .
$$

Here $j=0$ for scalar fields and tensor-type gravitational perturbations, $j=2$ for vector-type gravitational perturbations and $j=2 /(d-2), 2-2 /(d-2)$ for vectorand scalar-type electromagnetic perturbations, respectively. The coefficient $k_{d}$ can be determined analytically for given values of $d$ and $j$ 234. For electromagnetic perturbations in $d=5$, Eq. (103) is singular: this either means that there are no asymptotic QNM frequencies at all (a possibility first suggested in [101]) or that the asymptotic frequency is zero. Numerical results support the latter possibility [234.

The result above illustrates a general feature of the high-damping regime, which concerns the damping itself. It was shown by several authors [277, 278, 249] that for spacetimes with a single horizon, for all values of $l$ one has

$$
\omega_{I}=-2 \pi i n / T, \quad n \rightarrow \infty
$$

where $T$ is the Hawking temperature. General results for the oscillation frequencies were obtained in Ref. [279] for single-horizon spacetimes (see also 280] for BHs in two-dimensional dilaton gravity). An elegant unification of these results dealing with a rather general class of wave equations is given in Ref. 281] (but see Ref. [282] for words of caution on the generality of the results). A review on the highly damped regime of QNMs for several spacetimes can be found in Ref. [107.

Eikonal limit For $l \gg 1$, a WKB analysis of the Schwarzschild-Tangherlini perturbation equations yields [216, 208,

$$
\omega r_{+}=\Omega_{c}(l+d / 2-3 / 2-i(n+1 / 2) \sqrt{d-3}) .
$$

where $\Omega_{c}=\sqrt{d-3} / \sqrt{d-1}[2 /(d-1)]^{\frac{1}{d-3}}$ is the orbital angular velocity at the circular null geodesic [208].

Infinite-dimensional limit $(d \rightarrow \infty)$ To our knowledge, the interesting limit where the dimensionality $d$ tends to infinity has not been studied in much detail in the literature. Using a WKB analysis for scalar fields or gravitational tensor modes we find

$$
\omega r_{+}=d / 2-i \kappa \sqrt{d / 2}, \quad d \rightarrow \infty
$$

with $\kappa$ a factor of order unity depending on the perturbing field ( $\kappa=1,1 / \sqrt{2}$ for scalar fields and vector gravitational perturbations, respectively). BHs in higher dimensions 
are much better resonators, with a quality factor $Q \equiv \omega_{R} /\left[2 \omega_{I}\right]$ increasing as $\sqrt{d}$. As far as we know, no numerical studies are available to confirm this analytical prediction.

It is important to recall that black objects in higher dimensions may have other topologies beside the spherical one. One family consists of the so-called black strings, whose gravitational perturbations were studied by Gregory and Laflamme 283, 284 and Kudoh [285. Higher-dimensional "squashed" Kaluza-Klein BHs were considered in [286, and perturbations of brane-localized BHs were studied, for instance, in 287, 288, 289. Apart from specific noteworthy exceptions 290, 291, 292, 293, gravitational perturbations of higher-dimensional rotating solutions are yet to be understood. For a review on higher-dimensional BHs, see Ref. 34].

\section{The spectrum of asymptotically anti-de Sitter black holes}

\subsection{Schwarzschild anti-de Sitter black holes}

The metric (3) of an uncharged static BH in an (asymptotically) AdS spacetime has two parameters, $r_{0}$ and $L$, related, respectively, to the mass of the $\mathrm{BH}$ and the cosmological constant. The horizon radius $r_{+}$is the largest root of the equation $f(r)=0$. The Hawking temperature associated with the metric (3) is

$$
T=\left[d-3+(d-1) \xi^{2}\right] /(4 \pi \xi L),
$$

where $\xi=r_{+} / L$. For $d \geq 4$, this function has a characteristic minimum at $\xi_{\min }=\sqrt{(d-3) /(d-1)}$ with $T_{\min }=\sqrt{(d-1)(d-3)} / 2 \pi L$ (see Fig. 11).

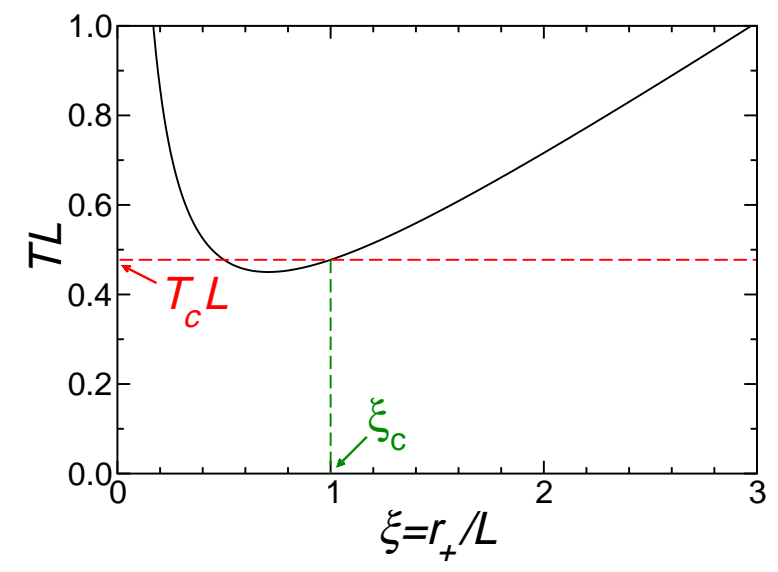

Figure 11. The (dimensionless) Hawking temperature $T L$ as a function of the (dimensionless) horizon radius $r_{+} / L$ for asymptotically AdS BHs (shown here for $d=5$ ). The horizontal line is the critical temperature of the Hawking-Page phase transition.

The specific heat of BHs with $\xi<\xi_{\min }\left(\xi>\xi_{\min }\right)$ is negative (positive). For $T<T_{\min }$, no $\mathrm{BH}$ solution exists, and the state with minimal free energy is "thermal AdS" (Euclidean $\mathrm{AdS} / \mathbb{Z}$ ). For $T>T_{\min }$, there are two solutions with the same temperature, "small" and "large" BHs. Small BHs have negative specific heat and are thermodynamically unstable. Large BHs exist in equilibrium with the heat bath. 
Moreover, for $\xi<1$, the minimum of the free energy still corresponds to thermal AdS, even for $T>T_{\min }$. Thus the stable ground state is given by large BHs with $\xi>1$ (and $\left.T>T_{c}=(d-2) / 2 \pi L\right)$. The (first order) transition between thermal AdS and a large AdS BH is known as the Hawking-Page phase transition [294. As shown by Witten [295], in the gauge-gravity duality this transition corresponds to a first order deconfinement transition in a dual thermal strongly coupled gauge theory on a sphere of radius $L$. These considerations can be extended to charged and rotating AdS BHs (see e.g. 296, 297] and references therein). The role of small AdS BHs in the gaugegravity duality is not well understood (see, however, Ref. 298]). The limit of extra large BHs with $r_{+} / L \rightarrow \infty$ in the appropriate scaling leads to black branes dual to strongly coupled gauge theories in flat space (see Section 8).

$\mathrm{BHs}$ in AdS in the context of the gauge-string duality attract considerable interest because they serve as a good laboratory for studying the most acute problems of a theory of gravity: the information loss paradox, $\mathrm{BH}$ singularities and some aspects of quantum gravity. Although we will not venture into this fascinating field in this review, in Section 8 we mention some of these topics which involve the use of QNMs.

Another peculiar feature of asymptotically AdS space is the "active role" played by its boundary. In AdS, null geodesics reach the boundary in finite coordinate time. One thus often refers to an asymptotically AdS space as a box, having in mind that AdS boundary conditions directly affect the bulk physics [299, 300, 301]. This should be contrasted with the asymptotically flat case, where the only physically relevant choice for the boundary conditions of the bulk fluctuations corresponds to outgoing waves at spatial infinity. In the gauge-gravity duality, the choice of the AdS boundary conditions is dictated by a holographic prescription [98, 302, 303, 304, see Section 8.

QNMs in asymptotically AdS backgrounds were first considered by Chan and Mann for a conformally coupled scalar field [305] (see also Ref. [306]). Subsequent interest was strongly motivated by the development of the AdS/CFT correspondence. An interesting albeit somewhat qualitative discussion of thermalization in AdS/CFT first mentioning QNMs appeared in Ref. 307. (see also the important work of Danielsson, Keski-Vakkuri and Kruczenski [308, 309]). Horowitz and Hubeny 92 explicitly pointed out the fundamental link between QNMs of a large AdS BH, which describe the background's relaxation into a final state, and the dual field theory, where they describe the approach to thermal equilibrium. Ref. 92 computed the QNMs of a minimally coupled massless scalar in intermediate or large AdS BHs for dimensions $d=4,5,7$. After this seminal work, a series of studies in four and higher dimensions led to a deeper understanding of QNMs in asymptotically AdS backgrounds.

The following is a brief overview of the QNMs of the four and higher-dimensional SAdS geometries. For more details we refer to the original works (see [92, 107, 130, 131, 310, 194, 311, 312, 313, 223, 314, 315, 316, 317, 318, 319, 320, 271, 321, 322, 323, 324. and references therein). The results below focus mostly on the large $\mathrm{BH}$ regime $r_{+} / L \gg 1$ and on Dirichlet boundary conditions, which are more relevant for holography. Other boundary conditions were investigated in Refs. [162, 163, 164, 165.

The weakly damped modes of large black holes The fundamental QNM frequencies for scalar field perturbations were first computed by Horowitz and Hubeny 92 . for intermediate and large BHs. In Refs. 130, 194 the analysis was extended to electromagnetic and gravitational perturbations. These works considered only $d=4$; the $d=5$ case was analyzed in Ref. [316], and half-integer spins were considered in Refs. 325, 326]. Approximate analytical solutions have been discussed 
in Refs. 312, 313, 314 with particular emphasis on the $d=5$ geometry.

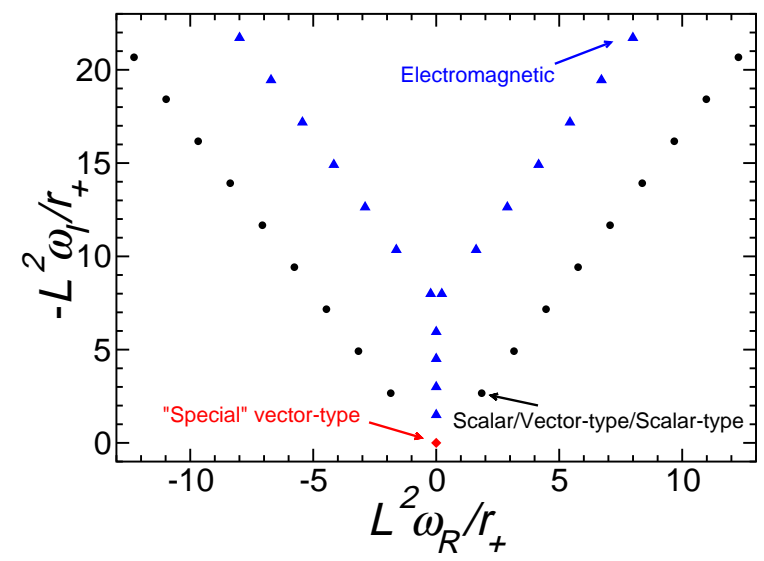

Figure 12. QNM frequencies for scalar $(s=0)$, electromagnetic $(s=1)$ and gravitational $(s=2)$ perturbations of large SAdS BH, computed for $r_{+} / L=100$ and $l=s$. In the large $\mathrm{BH}$ regime the frequencies scale with $r_{+} / L$ and are basically independent of $l$ for $l \ll r_{+} / L$. Furthermore, scalar-field, tensor-type and vector-type gravitational perturbations are nearly isospectral, except for a special mode belonging to the vector-type family and marked by a red diamond. For further details see 194, 311.

The weakly-damped QNM spectrum of a large $\left(r_{+} / L=100\right)$ SAdS BH in $d=4$ is shown in Fig. 12. Usually the QNM frequencies scale with the horizon radius, $\omega \propto r_{+} / L^{2}$ [92, 194], so the modes of any large $\mathrm{BH}$ can be obtained by rescaling appropriately the numbers in the figure. The exception to this rule is marked by a red diamond and will be discussed in more detail below. The frequencies of different perturbations are very similar: scalar perturbations and scalartype gravitational perturbations have basically the same spectra, while vector-type gravitational perturbations are displaced by one overtone relative to these two [194. The QNM frequencies are practically independent of $l$ for $l \ll r_{+} / L$; the large- $l$ limit is discussed below. The QNM spectrum for electromagnetic perturbations has a peculiar structure [130, 311, 194, 327, 328]: the real part of some modes asymptotes to zero when $r_{+} / L \rightarrow \infty$, and for the dominant mode $\omega_{I} L^{2} / r_{+} \rightarrow-1.5$.

The fundamental QNM for vector-type gravitational perturbations is extremely long-lived when compared to all other modes of other kinds of perturbations. This mode has an interesting interpretation in the gauge/gravity duality, discussed in Section 8 . The timescale of this long-lived mode is proportional to $r_{+}$, and the mode itself is easily computed numerically by a straightforward application of the series solution [130, 194. In a general $d$-dimensional SAdS geometry it is well described by

$$
r_{+} \omega_{n=0}^{\text {vector-type }}=-i(l-1)(l+d-2) /(d-1), \quad r_{+} / L \gg 1 .
$$

This was first observed numerically in Refs. 130, 194 for $d=4$, and in Ref. 316 for $d=5$. The four-dimensional result was later confirmed analytically by Miranda and Zanchin [315. Eq. (108) for general $d$ was derived by Siopsis [324, and we checked 
that it agrees with numerical results for $d=4$ up to $d=8$. In $d=4$ and for $r_{+} / L \gg l$, corrections to Eq. (108) can be found:

$$
r_{+} \omega_{n=0}^{\text {vector-type }}=-i(l-1)(l+2) / 3-0.0288 i l^{4} L^{2} / r_{+}^{2} .
$$

We estimate the uncertainty in the $l^{4}$ term to be about $5 \%$. Analytical calculations of these corrections have been done in the $r_{+} \rightarrow \infty$ limit [109, 329, 330] and are consistent with the numerical results above after proper identifications. In this limit the geometry becomes that of a black brane and the angular wavefunctions $Y_{l m}$ are replaced by $e^{i \vec{p} \vec{x}}$. For large $l, p$ one has the correspondence $L p=l$ [331]. In particular, it is found that in the large $l / L$ limit,

$$
\omega r_{+}=-\frac{i l^{2}}{3}-i L^{2} l^{4} \frac{9-9 \log 3+\sqrt{3} \pi}{162 r_{+}^{2}} \sim-\frac{i l^{2}}{3}-\frac{0.0281 L^{2} l^{4}}{r_{+}^{2}}
$$

in quite good agreement with the numerical fits. For similar high-order analytical corrections in higher-dimensional AdS backgrounds, see Refs. [109, 329, 330.

If Dirichlet boundary conditions are imposed at infinity, the scalar-type gravitational sector does not have such a long-lived mode. It was suggested that the preferred boundary conditions in the AdS/CFT framework are of Robin type, and in particular that the perturbations should not deform the metric on the AdS boundary [316, 163. Using Robin conditions, a long-lived mode for gravitational perturbations was discovered in Refs. 316, 163, 324.

The weakly damped modes of small black holes The series solution method, which works so well for large BHs, converges very slowly for small BHs [318, 310, 194. Recent results make use of Breit-Wigner type resonances in the scattering cross-section to study the very small BH regime [227, described briefly in Section 4.5. Small and large BHs have a very different behavior from a QNM perspective. This is related to the qualitatively different behavior of the potential in the two regimes, which is shown in the left panel of Fig. 13 for $s=0$ : for small BHs $\left(r_{+} / L<1\right)$ the potential develops a well capable of sustaining trapped, long-lived modes, corresponding to quasi-stationary states in quantum mechanics [332, 331, 227]. In the right panel of Fig. 13 we plot the QNMs of scalar fields for different $\mathrm{BH}$ sizes and $l=0$, 1. In this limit (and under the assumption that $M \omega_{R} \ll 1$ ) it is possible to prove that [333]

$$
\begin{aligned}
& \omega_{R} L \simeq l+3+2 n-k_{l n} r_{+} / L \\
& \omega_{I} L \simeq-\gamma_{0}(l, n)(l+3+2 n)\left(r_{+} / L\right)^{2 l+2} / \pi .
\end{aligned}
$$

The constant $\gamma_{0}(l, n)(n=0,1,2, \ldots)$ and selected values of the constants $k_{l 0}$ can be found in Ref. [227]. The analytic prediction is consistent with numerical results. Likewise, for gravitational perturbations with $r_{+} / L \ll 1$ one finds

$$
\begin{aligned}
& \omega_{R} L \simeq l+2+2 n-c_{l n} r_{+} / L, \\
& \omega_{I} L \simeq-\gamma_{2}(l, n)(l+2+2 n)\left(r_{+} / L\right)^{2 l+2},
\end{aligned}
$$

where the constants $c_{l n}$ and $\gamma_{2}(l, n)$ can be found in Refs. [334, 227]. In conclusion: for scalar field and gravitational perturbations of small SAdS BHs the damping timescale $\omega_{I} L \sim\left(r_{+} / L\right)^{2 l+2}$, and the oscillation frequencies approach the pure AdS value in the limit $r_{+} / L \rightarrow 0$ (see [310, 227] for more details). To our knowledge, generalizations of these results to charged, higher-dimensional and rotating AdS BHs are still lacking. 

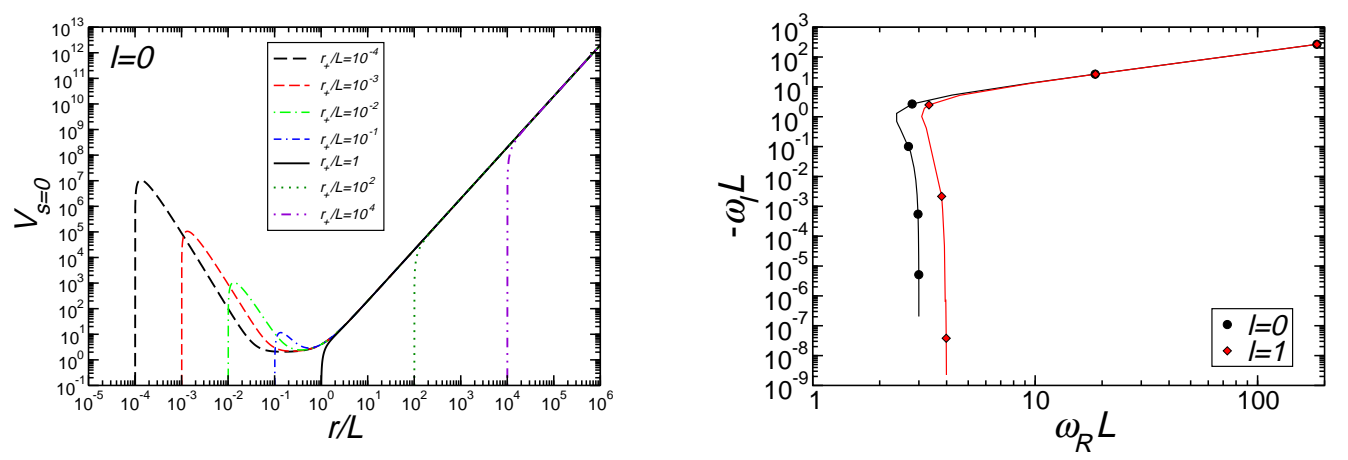

Figure 13. Left: Potential for scalar field propagation of $l=0$ modes in a SAdS background, for different values of the $\mathrm{BH}$ size $r_{+} / L$. A local maximum (and a potential well) only exist for small BHs. Right: Track described by the fundamental scalar field QNMs with $l=0$ and $l=1$ as we vary the BH size $r_{+} / L$. Counterclockwise starting from the top-right of the figure we marked the points corresponding to different decades in $r_{+} / L\left(r_{+} / L=10^{2}, 10^{1}, 10^{0}, 10^{-1}, \ldots\right)$. Modes with different $l$ coalesce in the large $\mathrm{BH}$ regime, as long as $l \ll r_{+} / L$.

The highly-damped modes The large-damping regime $(n \gg 1)$ can be studied using the monodromy method for both small and large AdS BHs [107, 223]. For $n \gg 1$ and $r_{+} / L \gg 1$ (large $\mathrm{BHs}$ ) the QNMs are given by

$$
\frac{\omega L^{2}}{r_{+}}=(d-1) \sin \left(\frac{\pi}{d-1}\right) e^{\frac{i \pi}{d-1}}\left[n+\frac{d+1}{4}-i \frac{\log 2}{2 \pi}\right] .
$$

Results for arbitrarily sized BHs can be found in Refs. [107, 223]. Eq. (115) describes scalar fields, gravitational tensor and gravitational vector-type perturbations. It yields $\omega L^{2} / r_{+} \approx(1.299+2.250 i) n+(0.573+0.419 i)$ for $d=4$ and $\omega L^{2} / r_{+} \approx$ $(2+2 i) n+3.22064+2.77936$ for $d=5$, in good agreement with numerical results for $d=4$ [194] and $d=5$ [316]. For large SAdS BHs the asymptotic high-damping regime is approached very quickly, so Eq. (115) describes fairly well even weakly-damped modes. There is some disagreement concerning the offset for scalar-type gravitational perturbations [107.

An asymptotic analysis based on the monodromy method was recently used to predict the existence of a new family of modes [335. So far, this new part of the spectrum has not been confirmed by numerical studies. In Ref. 336] the authors discuss the interesting possibility of describing the highly damped QNM regime in terms of geodesics of the SAdS spacetime.

The eikonal limit of large black holes In asymptotically AdS spacetimes the eikonal limit is especially interesting, since large-l modes can be very long-lived [92, 331. A WKB analysis [331] and numerical investigations [337] show that for scalar field perturbations, $r_{+} / L \gg 1$ and $l \gg 1$

$$
\omega L=l+\Pi_{n}\left(r_{+} / L\right)^{\frac{2 d-2}{d+1}} l^{-\frac{d-3}{d+1}},
$$




$$
\Pi_{n} \equiv \frac{1}{2}\left(\sqrt{\pi}(d-1)\left[\frac{d+1}{2}+2 n\right] \frac{\Gamma(3 / 2+1 /(d-1))}{\Gamma(1 /(d-1))}\right)^{\frac{2 d-2}{d+1}} e^{-\frac{i 2 \pi}{d+1}} .
$$

So large- $l$ modes are very long-lived, and they could play a prominent role in the BH's response to generic perturbations. This is at variance with the asymptotically flat case, where the damping timescale is roughly constant as $l$ varies. Notice also that the scaling with the BH size differs from that of the weakly-damped and highly-damped modes. Other types of perturbations also display a similar qualitative behavior [337.

The eikonal limit of small black holes For scalar and electromagnetic perturbations of small BHs the potential for wave propagation for $l \gg 1$ develops a minimum, potentially supporting quasi-stationary states, i.e. long-lived modes (see Fig. 13). Define $r_{b}>r_{c}$ to be the two real zeros (turning points) of $\omega_{R}^{2}-p^{2} f / r^{2}=0$. Then the real part of a class of long-lived modes in four spacetime dimensions is given by the WKB condition

$$
2 \int_{r_{b}}^{\infty} \frac{\sqrt{r^{2} \omega_{R}^{2}-p^{2} f}}{r f} d r=\pi\left(2 n+1+\frac{3}{2}\right),
$$

where $p=l+d / 2-3 / 2$. Their imaginary part is given by

$$
\omega_{I}=\frac{\gamma \Gamma}{8 \omega_{R}}, \quad \log \Gamma=2 i \int_{r_{b}}^{r_{c}} \frac{\sqrt{r^{2} \omega_{R}^{2}-p^{2} f}}{r f} d r .
$$

The prefactor $\gamma$, not shown in Ref. 331, can be obtained by standard methods as shown in Ref. [227, where these results are also supported by numerical calculations.

\subsection{Reissner-Nordström and Kerr anti-de Sitter black holes}

The analysis of QNMs of large RNAdS BHs was performed in Ref. 338, 339] for weakly damped modes of a massless scalar field, and later extended to charged scalar fields 340. Scalar field, electromagnetic and gravitational perturbations of RNAdS BHs were analyzed and compared in Ref. [311. Half-integer spins were studied in Refs. 341, 342. Gravitational perturbations of higher-dimensional charged solutions were considered in 343, and the highly-damped regime was explored analytically by Natário and Schiappa [107.

Ref. 311 pointed out some interesting facts: (i) a near-isospectrality of different classes of perturbations holds for large BHs, but it breaks down as $r_{+} / L$ decreases, i.e. in the small BH limit; (ii) the imaginary part of the purely damped modes found for electromagnetic and vector-type gravitational perturbations tends to zero as the charge $Q$ tends to the extremal value $Q_{\text {ext }}$, possibly pointing to a marginal instability of extremal RNAdS BHs; (iii) for all kinds of perturbations, the real part of the fundamental QNM frequency, $L \omega_{R}$, has a minimum for $Q / Q_{\text {ext }} \simeq 0.366$, followed by a maximum at $L \omega_{R} \simeq 0.474$. A reanalysis of massless scalar field perturbations found that the imaginary part of scalar QNMs also tends to zero in the extremal limit 344. However, comparing time-evolutions of the field with results from the Horowitz-Hubeny series solution, Wang et al. 344 found that the BH response turns from a standard, oscillatory QNM-type decay below some critical value of the charge $\left(Q<Q_{\text {crit }} \simeq 0.3895 Q_{\text {ext }}\right)$ to a non-oscillatory behavior characterized by a purely imaginary QNM frequency for $Q>Q_{\text {crit }}$. Furthermore, these authors suggested that the potential marginal instability proposed in Ref. 311 does not pose a threat for extremal RNAdS BHs, because (at least for scalar perturbations) the asymptotic field 
in the near-extremal limit is dominated by a power-law tail of the type first analyzed by Price [171. The implications of these findings in the context of the AdS/CFT correspondence deserve further investigation.

There are few studies of QNMs of rotating BHs in AdS backgrounds. The formalism to handle perturbations of these spacetimes was laid down by Chambers and Moss 345. Giammatteo and Moss [346] considered axially symmetric QNMs of large Kerr-AdS BHs. These spacetimes behave as a BH in a box. Superradiant amplification of incident waves at the expense of the BH's rotational energy can then produce instabilities in the non-axisymmetric modes of small Kerr AdS BHs 347, 333, 334, 348, 349, 292, 350]: this is an interesting example of the "black hole bomb" first investigated by Press and Teukolsky [351].

\subsection{Toroidal, cylindrical and plane-symmetric anti-de Sitter black holes}

The uniqueness theorems that apply for asymptotically flat BHs (see e.g. [39]) can be evaded when the cosmological constant is non-zero: solutions have been found with the topology of a cylinder, of a plane or of a doughnut [122, 123, 124, 125, 126, 127. Defining $f(r)=r^{2} / L^{2}-4 M / r$, these spacetimes are described by

$$
d s^{2}=f d t^{2}-f^{-1} d r^{2}-r^{2} d z^{2}-r^{2} d \phi^{2} .
$$

The range of the coordinates $z$ and $\phi$ dictates the topology of the BH spacetime. For a $\mathrm{BH}$ with toroidal topology the coordinate $z$ is compactified such that $z / L$ ranges from 0 to $2 \pi$, and $\phi$ ranges from 0 to $2 \pi$ as well. For the cylindrical BH, or black string, the coordinate $z$ has range $-\infty<z<\infty$, and $0 \leq \phi<2 \pi$. For the planar BH, or black membrane, the coordinate $\phi$ is further decompactified $(-\infty<L \phi<\infty)$. For the torus, $M$ is related to the system's ADM mass; for the cylinder, to the mass per unit length of a constant- $z$ line; and for the plane, to the mass per unit area of the $(\phi, z)$ plane $[122,123,124,125,126,127$.

A formalism to handle electromagnetic and gravitational perturbations of these spacetimes was developed and used to investigate numerically the QNMs of scalar, electromagnetic and gravitational perturbations in Ref. [131. A thorough analysis by Miranda and Zanchin [315, 327] confirmed and extended the results of Ref. [131].

\section{The spectrum of asymptotically de Sitter and other black holes}

\subsection{Asymptotically de Sitter black holes}

$\mathrm{BH}$ in a dS background have a rich structure. Consider an uncharged spherically symmetric $\mathrm{BH}$, the $\mathrm{SdS}_{d}$ solution,

$$
d s^{2}=f d t^{2}-f^{-1} d r^{2}-r^{2} d \Omega_{d-2}^{2},
$$

where $f(r)=1-r^{2} / L^{2}-r_{0}^{d-3} / r^{d-3}, d \Omega_{d-2}^{2}$ is the metric of the $(d-2)-$ sphere, and $r_{0}$ is related to the mass $M$ of the spacetime by $M=(d-2) A_{d-2} r_{0}^{d-3} /(16 \pi)$. Depending on the value of $r_{0} / L^{2}$, in general this solution has two horizons, the event and the cosmological horizon. When the cosmological and event horizons coalesce one has a so-called extremal $\mathrm{SdS}_{d}$ solution, which is related to a topologically different solution, known as the Nariai spacetime [199, 200, 201, 196]. 
Weakly damped modes The first calculations of the fundamental gravitational modes were done by Mellor and Moss [129] for Reissner-Nordström-de Sitter (RNdS) BHs, using numerical techniques. Brady et al. 352 complemented this study through a numerical time evolution of scalar fields in the SdS spacetime. Time evolutions were also performed in Ref. 353, where the results were compared against WKB predictions (see also Ref. [274]). Approximations to the correct, lowest order QNMs were considered in Refs. [162] $(s=2, \mathrm{SdS})$, 354] $(s=0, \mathrm{SdS})$, 355] $(s=1 / 2, \operatorname{RNdS})$ and 356] $(s=2, \mathrm{RNdS})$, where the true potential was approximated by a PöschlTeller potential. Cardoso and Lemos [191] showed that the true potential reduces to the Pöschl-Teller potential for near extremal SdS geometries, which explains why previous results based on the Pöschl-Teller approximation gave accurate predictions for the QNMs. The results in [191] were later generalized to $d$-dimensional RNdS BHs 197. Other analytical results used the WKB approximation for spins $s=0,1,2,1 / 2$ in the vicinity of SdS BHs 357.

Highly-damped modes The highly-damped limit, where the imaginary part is much larger than the real part, was studied numerically by Yoshida and Futamase 238] for near-extremal uncharged BHs $(s=1,2)$ and in the general case in Ref. [358, 239]. The results were confirmed analytically in Refs. [223] $(s=0,1,2 \mathrm{SdS})$ and [107] $(s=0,1,2$ $\mathrm{RNdS})$.

Eikonal limit In the large-l limit, WKB techniques [190, 357] yield

$$
3 \sqrt{3} M \omega=\sqrt{1-27 M^{2} / L^{2}}[l+1 / 2-i(n+1 / 2)] .
$$

QNMs in the eikonal limit can be interpreted as perturbations of unstable circular null geodesics 208. For studies on charged and rotating BHs in dS backgrounds, see 359 and references therein.

\subsection{Black holes in higher-derivative gravity}

Among all possible theories of gravity with higher derivative terms, theories modified by the addition of a Gauss-Bonnet term $\mathcal{R}_{G B}=R_{\mu \nu \rho \sigma} R^{\mu \nu \rho \sigma}-4 R_{\mu \nu} R^{\mu \nu}+R^{2}$, are particularly attractive and have been considered by many authors [360]. There are simple BH solutions in these theories [361, 362, 363, 364, 365, 366, 367, 368, 369, 370, whose perturbations were studied in a series of works by Dotti and Gleiser 371, 372, 373, Moura and Schiappa 360. and Takahashi and Soda 374. In four dimensions the Gauss-Bonnet term is a total divergence, and yields upon integration a topological invariant, being therefore equivalent to Einstein's theory. Therefore these theories are interesting in higher dimensions only. Non-trivial four-dimensional scenarios can be accomplished by coupling the Gauss-Bonnet term to a dilaton 367: the resulting theory also arises from the low-energy limit of certain string theories 360]. Perturbations of $\mathrm{BH}$ spacetimes in these scenarios were considered in Refs. 367, 375, 365.

QNMs of BHs in these theories were first investigated by Iyer et al. [376], who used a WKB approach to study scalar perturbations. QNMs of these spacetimes were also studied through a WKB approach [377, 378, 379, and numerically [380, 377. for the low-lying modes. The highly-damped regime was analytically explored in Ref. [282. The eikonal limit was considered by Konoplya 380] and interpreted in terms of circular null geodesics in Ref. [208. 


\subsection{Braneworlds}

Braneworld scenarios, where the standard model lives in a four-dimensional brane embedded in a higher dimensional spacetime, have been a popular research topic in the last decade. The extra dimensions can be compact 381, 382 or even infinite, flat 383] or curved [384, 385. BH solutions in these theories are extremely difficult to find (see for instance Ref. [386, 387] for a discussion), some solutions are known perturbatively in some regimes. For instance, in the case of flat compact extra dimensions, Tangherlini BHs should be a good approximation to a static $\mathrm{BH}$ solution as long as the horizon radius is much smaller than the size of the extra dimension 386]. In these scenarios the standard model is localized on the brane and therefore BH oscillations are non-trivial, especially for QNMs of standard-model fields 388, 389, 390, 391, 392, 393.

\subsection{Black holes interacting with matter}

Astrophysical BHs are not expected to be in complete isolation, so it is important to understand how QNMs change when BHs interact with the surrounding environment. Leung et al. [394, 395] investigated how the low-order QNMs of a BH are affected by a small amount of matter. For such a BH, in the static, spherically-symmetric case, Medved et al. [277, 278, proved that highly-damped QNMs depend only on the surface gravity. Specific models for BHs interacting with matter were constructed by several authors. A popular cosmological scenario invokes the existence of dynamical vacuum energy ("quintessence", see e.g. [396, 397]) or phantom fields [398, 399] to explain the acceleration of the universe. The QNMs of BHs with quintessence or phantom fields have been investigated in Refs. 400, 401, 402, 403, 404, 405] and 406, respectively.

\section{Quasinormal modes and the gauge-gravity duality}

In this Section, we review a particular entry in the gauge-gravity duality dictionary directly related to QNMs. It turns out that quasinormal spectra of asymptotically $\mathrm{AdS}_{d+1}$ and more general backgrounds correspond to poles of the (retarded) thermal correlators of dual $d$-dimensional strongly interacting quantum gauge theories. The lowest quasinormal frequencies of black branes have a direct interpretation as dispersion relations of hydrodynamic excitations in the dual theory. More information on near-equilibrium properties of $\mathrm{BHs}$ and black branes and their holographic interpretation can be found in the reviews [7, 117, 36, 37, 38.

\subsection{The duality}

The discovery 407] and subsequent studies of $D$-branes in string theory led to the concept of gauge-string duality. In the original example of the duality, known as the AdS/CFT correspondence [6], the full type IIB string theory on the background $\mathrm{AdS}_{5} \times S^{5}$ (five-dimensional AdS space times a five-sphere) was conjectured $\$$ to be equivalent to the specific supersymmetric gauge theory, $\mathcal{N}=4$ $S U\left(N_{c}\right)$ supersymmetric Yang-Mills theory (SYM) in flat four-dimensional spacetime. The equivalence is understood as an equivalence of quantum partition functions. In

$\S$ During the last decade, the AdS/CFT correspondence and, more generally, the gauge-string duality, survived numerous, often very non-trivial tests of validity. At the moment, there is very little doubt, if any, that the conjecture is valid. 
quantum field theory, there is very strong evidence supporting the claim that $\mathcal{N}=4$ SYM is a conformal field theory (CFT), with the beta-function identically equal to zero and coupling constant $g_{Y M}$ being independent of the energy scale. The two parameters characterizing $\mathcal{N}=4 \mathrm{SYM}$ are the 't Hooft coupling $\lambda \equiv g_{Y M}^{2} N_{c}$ and the number of colors $N_{c}$. In the AdS/CFT correspondence, these parameters are mapped into the string theory parameters $L$ and $g_{s}$ :

$$
g_{Y M}^{2} N_{c} \sim g_{s} N_{c} \sim L^{4} / l_{s}^{4}, \quad N_{c} \sim L^{4} / l_{P}^{4}
$$

where $L$ is the parameter of $\mathrm{AdS}_{5}$ and the radius of the five-sphere, $g_{s}$ is the string coupling and $l_{s}, l_{P}$ are the string and Planck lengths, respectively. The full quantum string theory on $\mathrm{AdS}_{5} \times S^{5}$ is poorly understood. However, its low-energy limit, type IIB supergravity, has been extensively studied since the $1980 \mathrm{~s}$. Restricting duality to the supergravity limit of the full string theory restricts the values of the gauge theory parameters to $g_{Y M}^{2} N_{c} \gg 1, N_{c} \gg 1$. Thus the gauge theory at large values of coupling and large $N_{c}$ is effectively described by classical gravity in the AdS background.

Following the original example of AdS/CFT correspondence, many more dual pairs have been discovered, including those involving non-supersymmetric and nonconformal theories. Gauge-string duality thus includes the original AdS/CFT correspondence and all its "non-conformal" and "non-AdS" generalizations, often commonly referred to as "AdS/CFT". The gauge-string duality in the supergravity approximation is known as the gauge-gravity duality. The duality provides a quantitative correspondence between classical gravity in ten (or five) dimensions and a gauge theory (in the limit $g_{Y M}^{2} N_{c} \gg 1, N_{c} \gg 1$ ) in flat four-dimensional spacetime. Such a correspondence between higher-dimensional gravity and lower-dimensional nongravitational theory is often referred to as "holography". The gauge-gravity duality serves as a quantitative example of the "holographic principle" proposed by 't Hooft and Susskind 408, 409.

Since classical higher-dimensional gravity is holographically encoded into the dual gauge theory's properties, one may wonder about the gauge theory interpretation of the QNM spectrum. The short answer, conjectured in [94], established in [98], and further generalized in [109] and many subsequent publications, is that the $Q N M$ spectrum of the fluctuation $\delta \phi$ of a higher-dimensional gravitational background coincides with the location of the poles of the retarded correlation function of the gauge theory operator $\mathcal{O}$ dual to the fluctuation $\delta \phi$. In the rest of this Section, we elaborate on this statement and provide some explicit examples.

The main ingredient of the gauge-gravity duality is the ten-dimensional (super)gravity background characterized by the values of the metric and other supergravity fields such as the dilaton, the axion and various tensor fields. The background fields must satisfy supergravity equations of motion. (In most cases, only bosonic supergravity fields are considered, thus eliminating the need for the prefix "super".) For example, the near-horizon limit of the black three-brane background, which is the basic ingredient of the AdS/CFT duality at finite temperature, consists of the metric

$$
d s_{10}^{2}=\frac{r^{2}}{L^{2}}\left[-f d t^{2}+d x^{2}+d y^{2}+d z^{2}\right]+\frac{L^{2}}{r^{2}} f^{-1} d r^{2}+L^{2} d \Omega_{5}^{2},
$$

where $f(r)=1-r_{0}^{4} / r^{4}$, and the Ramond-Ramond five-form field,

$$
F_{5}=-\frac{4 r^{3}}{L^{4}}(1+*) d t \wedge d x \wedge d y \wedge d z \wedge d r
$$


with all other fields vanishing. According to the gauge/gravity correspondence, the background (123)-(124) with non-extremality parameter $r_{0}$ and Hawking temperature $T=r_{0} / \pi L^{2}$ is dual to $\mathcal{N}=4 S U\left(N_{c}\right)$ SYM at finite temperature $T$ in the limit of $N_{c} \rightarrow \infty, g_{Y M}^{2} N_{c} \rightarrow \infty$. The $\mathcal{N}=4 \mathrm{SYM}$ is defined in Minkowski space with coordinates $t, x, y, z$. The fifth (radial) coordinate $r$ of the dual metric (123) plays the role of the energy scale in the gauge theory (with the boundary at $r \rightarrow \infty$ corresponding to the ultraviolet in the gauge theory), and the five-sphere describes internal degrees of freedom associated with the $R$-symmetry group $S U(4)$ specific to theories with $\mathcal{N}=4$ supersymmetries.

Quite often, the internal degrees of freedom are of less interest, and the background can be dimensionally reduced from ten to five dimensions. For the metric (123), the result of such a reduction is the five-dimensional Schwarzschild-AdS metric with translationally invariant horizon

$$
d s_{5}^{2}=\frac{r^{2}}{L^{2}}\left[-f d t^{2}+d x^{2}+d y^{2}+d z^{2}\right]+\frac{L^{2}}{r^{2}} f^{-1} d r^{2},
$$

obeying Einstein's equations $R_{\mu \nu}=2 \Lambda / 3 g_{\mu \nu}$ in a five-dimensional space with cosmological constant $\Lambda=-6 / L^{2}$. Thus the gauge-gravity duality often appears as a correspondence involving the five-dimensional (super)gravity bulk and the fourdimensional boundary gauge theory. One should always remember, however, that all five-dimensional fields and their fluctuations have ten-dimensional origin.

As a remark, we note that the metric (123) with translationally invariant horizon can be obtained from the Schwarzschild-AdS BH metric

$$
d s_{5}^{2}=-f d t^{2}+\frac{d r^{2}}{f}+r^{2} d \Omega_{3}^{2}, \quad f=1+\frac{r^{2}}{L^{2}}-\frac{r_{0}^{4}}{r^{2} L^{2}}
$$

by rescaling $r \rightarrow \lambda^{1 / 4} r, r_{0} \rightarrow \lambda^{1 / 4} r_{0}, t \rightarrow \lambda^{-1 / 4} t$, and taking the limit $\lambda \rightarrow \infty$ while simultaneously blowing up the sphere

$$
L^{2} d \Omega_{3}^{2} \rightarrow \lambda^{-1 / 2}\left(d x^{2}+d y^{2}+d z^{2}\right) .
$$

Similar rescalings for more general metrics can be found in [296, 410]. This difference between black hole and black brane metrics leads to the fact that black brane QNM spectra are functions $\omega=\omega(q)$ of the continuous parameter $q$ (rather than a discrete parameter $l$ ), where $q$ is the momentum along the translationally invariant directions.

\subsection{Dual quasinormal frequencies as poles of the retarded correlators}

Quantitatively, the gauge-string (gauge-gravity) duality is the equivalence of the partition functions

$$
Z_{Y M}[J]=\left\langle e^{-\int J \mathcal{O} d^{4} x}\right\rangle_{Y M} \equiv Z_{\text {string }}[J] \simeq e^{-S_{\text {grav }}[J]},
$$

where the semiclassical approximation on the right-hand side corresponds to passing from the gauge-string to the gauge-gravity duality in the appropriate limit (e.g. in the limit $N_{c} \rightarrow \infty, g_{Y M}^{2} N_{c} \rightarrow \infty$ for $\mathcal{N}=4 \mathrm{SYM}$ ). The equivalence (128) means that the classical gravity action effectively serves as a generating functional for correlation functions of gauge-invariant operators $\mathcal{O}$ in the dual gauge theory. On the gravity side, the role of $J$ for a given operator $\mathcal{O}$ is played by the boundary value $\delta \phi_{0}$ of

\| The cosmological constant in five dimensions arises as a result of the dimensional reduction of the five-form (124) on $S^{5}$. 
the background fluctuation $\delta \phi$ (for a moment, we are ignoring all indices the field $\delta \phi$ might have). For example, the boundary value of the background metric fluctuation $h_{\mu \nu}$ plays the role of $J$ in computing the correlators of the energy-momentum tensor $T_{\mu \nu}$ in a four-dimensional gauge theory (see Table 22).

The recipe for applying the equivalence (128) is the following. To compute the correlators of a gauge-invariant operator $\mathcal{O}$, one has to

- identify the dual fluctuation field $\delta \phi$ associated with $\mathcal{O}$

- solve the linearized bulk equations of motion satisfied by $\delta \phi$ with the boundary condition $\delta \phi \rightarrow \delta \phi_{0} \equiv J$

- using this solution, compute the on-shell supergravity action $S_{\text {grav }}[J]$ as a functional of $\delta \phi_{0} \equiv J$

- compute the correlators in the usual field theory sense by taking functional derivatives of $\exp \left(-S_{\text {grav }}[J]\right)$ with respect to $J$.

The recipe given above is sufficient for computing Euclidean correlation functions from dual gravity. For Minkowski space correlators, there are subtleties resolved in 98, 302, (recent work on the Lorentzian AdS/CFT includes [411, 304]). Ref. 98] contains an exact prescription on how to compute the Minkowski space two-point functions from fluctuations of a dual gravity background. As a byproduct, the prescription establishes a one-to-one correspondence between poles of the retarded quantum field theory correlators and QNM spectra of the dual background. Indeed, the dual gravity fluctuation field $\delta \phi(r, t, x, y, z)$ associated with the operator $\mathcal{O}$, whose retarded two-point function $G^{R}$ we are interested in, satisfies an ordinary linear secondorder differential equation with respect to the radial coordinate $r$. The fluctuation's dependence on the "usual" four-dimensional space-time coordinates $t, x, y, z$ in the bulk is typically trivial, allowing one to Fourier transform with respect to them:

$$
\delta \phi(r, t, x, y, z)=\int \frac{d \omega d \mathbf{q}}{(2 \pi)^{4}} e^{-i \omega t+i \mathbf{q x}} \delta \phi(r, \omega, \mathbf{q}) .
$$

Note that at finite temperature the Lorentz invariance is broken, thus the components $\omega, \mathbf{q}$ of the four-momentum are independent variables. For theories with unbroken rotation invariance, the fluctuation will depend on the magnitude of the threemomentum $q=|\mathbf{q}|$, and one can conveniently choose $\mathbf{q}$ along the direction $z$ of the brane, with the four-momentum being given by $(\omega, 0,0, q)$. The ODE satisfied by the fluctuation $\delta \phi(r, \omega, q)$ typically has singular points at the horizon $r=r_{0}$ and at the boundary $r \rightarrow \infty$. The solution $\delta \phi(r, \omega, q)$ satisfying the incoming wave boundary condition at the horizon can be written near the boundary as

$$
\delta \phi(r, \omega, q)=\mathcal{A}(\omega, q) r^{-\Delta_{-}}(1+\cdots)+\mathcal{B}(\omega, q) r^{-\Delta_{+}}(1+\cdots),
$$

where $\Delta_{+}, \Delta_{-}$are the exponents of the ODE at $r=\infty$ (these exponents are related to the conformal dimension of the operator $\mathcal{O}$ ), and ellipses denote higher powers of $r$. In most cases, fields can be redefined so that $\Delta_{+}>0, \Delta_{-}=0$. Applying the gaugegravity duality recipe for Minkowski correlators, for the retarded two-point function of the operator $\mathcal{O}$ one finds

$$
G^{R}(\omega, q) \sim \frac{\mathcal{B}}{\mathcal{A}}+\text { contact terms } .
$$

I The second boundary condition on the fluctuation $\delta \phi$ is either a regularity condition (e.g. for zerotemperature global AdS space) or the incoming (outgoing) wave boundary condition. For metrics with horizons the incoming (outgoing) wave condition corresponds to computing the retarded (advanced) correlators in the boundary theory. 
Table 2. The correspondence between the boundary gauge theory operators and the dual five-dimensional gravity bulk fields.

\begin{tabular}{|c||c|}
\hline Gauge theory operator $\mathcal{O}$ & Dual gravity fluctuation \\
\hline energy-momentum tensor $T_{\mu \nu}$ & metric fluctuation $h_{\mu \nu}$ \\
conserved current $J_{\mu}$ & Maxwell field $A_{\mu}$ \\
$\operatorname{Tr} F_{\mu \nu}^{2}$ & minimally coupled massless scalar $\varphi$ \\
\hline
\end{tabular}

Zeros of the coefficient $\mathcal{A}$ correspond to the poles of $G^{R}(\omega, q)$. On the other hand, from the general relativity point of view, the condition $\mathcal{A}=0$ (for $\Delta_{-}=0$ this is just the Dirichlet boundary condition) defines the QNM spectrum of the fluctuation $\delta \phi$. Thus, all the information about the poles of the retarded correlators of a quantum field theory with a gravity dual description is encoded in the QNM spectra of the dual gravity fluctuations. This statement is a useful entry in the gauge-gravity duality dictionary, since the poles of thermal correlators carry important information about transport properties and excitation spectra of the theory. Consider, for illustration, the relatively simple case of a two-dimensional CFT at finite temperature dual to the $(2+1)$-dimensional BTZ BH background. The retarded two-point function of the operator of conformal dimension $\Delta=2$ at finite temperature is given by 98

$$
G^{R} \sim \frac{\omega^{2}-q^{2}}{4 \pi^{2}}\left[\psi\left(1-i \frac{\omega-q}{4 \pi T}\right)+\psi\left(1-i \frac{\omega+q}{4 \pi T}\right)\right],
$$

where $\psi(z)=\Gamma^{\prime}(z) / \Gamma(z)$; we put $T_{L}=T_{R}$ and ignored the constant prefactor for simplicity. The correlator (132) has infinitely many poles in the complex frequency plane, located at

$$
\omega_{n}= \pm q-i 4 \pi T(n+1), \quad n=0,1,2, \ldots .
$$

These poles are precisely the BTZ quasinormal frequencies [305, 95, 412].

The role of QNM spectra as frequencies defining relaxation times in the dual thermal field theory was realized and discussed in early publications [307, 92, 309. Birmingham, Sachs and Solodukhin 94 were the first to note explicitly that the QNM spectrum of the BTZ BH coincides with the poles of the retarded correlators of the dual $(1+1)$-dimensional CFT. With the appearance of the Minkowski AdS/CFT recipe for the retarded correlators [98, the relationship between QNMs and the poles of the correlators has been established quantitatively first for the scalar [98, 99] and later for general fluctuations [100, 109. In the case of non-scalar fluctuations, considering gauge-invariant combinations of fluctuating fields is especially useful [109, although this is not the only possible approach [413, 414].

A word of caution is necessary. As we have seen, asymptotically AdS spacetimes offer a variety of choices for the boundary conditions at spatial infinity. Not all such choices produce QNM spectra which have a meaningful interpretation in the dual quantum field theory. In order to say that a computed QNM corresponds to a pole of a dual theory correlator, one has to analyse the bulk fluctuation along the lines leading to Eq. (131) to establish the precise form of the boundary condition.

\subsection{The hydrodynamic limit}

The most interesting results for QNM spectra with a dual field theory interpretation are obtained for five-dimensional gravitational backgrounds (note, however, the 
growing body of work on the AdS-Condensed Matter Theory correspondence [37, 38, where, for the purposes of studying $(2+1)$-dimensional condensed matter systems, one is interested in $(3+1)$-dimensional gravitational backgrounds). For example, the poles of the retarded thermal two-point function

$$
G_{\mu \nu, \lambda \sigma}^{R}(\omega, q)=-i \int d^{4} x e^{-i q \cdot x} \theta(t)\left\langle\left[T_{\mu \nu}(x), T_{\lambda \sigma}(0)\right]\right\rangle_{T}
$$

of the stress-energy tensor in four-dimensional $\mathcal{N}=4 S U\left(N_{c}\right)$ SYM in the limit $N_{c} \rightarrow \infty, g_{Y M}^{2} N_{c} \rightarrow \infty$ are given by the QNM frequencies of the gravitational perturbation $h_{\mu \nu}$ of the metric (125). By symmetry, the perturbations are divided into three groups 415, 109]. Indeed, since the dual gauge theory is spatially isotropic, we are free to choose the momentum of the perturbation along, say, the $z$ direction, leaving the $O(2)$ rotational symmetry of the $(x, y)$ plane intact. The perturbations $h_{\mu \nu}(r, t, z)$ are thus classified according to their transformation properties under $O(2)$. Following [15, 109, we call them the scalar $\left(h_{x y}\right)$, shear $\left(h_{t x}, h_{z x}\right.$ or $\left.h_{t y}, h_{z y}\right)$ and sound $\left(h_{t t}\right.$, $\left.h_{t z}, h_{z z}, h_{x x}+h_{y y}\right)$ channels. (Here we partially fixed the gauge by requiring $h_{\mu r}=0$.) The $O(2)$ symmetry ensures that the equations of motion for perturbations of different symmetry channels decouple. Linear combinations of perturbations invariant under the (residual) gauge transformations $h_{\mu \nu} \rightarrow h_{\mu \nu}-\nabla_{\mu} \xi_{\nu}-\nabla_{\nu} \xi_{\mu}$ form the gauge-invariant variables

$$
\begin{aligned}
& Z_{1}=q H_{t x}+\omega H_{z x}, \\
& Z_{2}=q^{2} f H_{t t}+2 \omega q H_{t z}+\omega^{2} H_{z z}+\left[q^{2}(2-f)-\omega^{2}\right] H, \\
& Z_{3}=H_{x y}
\end{aligned}
$$

in the shear, sound and scalar channels, respectively (here $H_{t t}=L^{2} h_{t t} / r^{2} f, H_{i j}=$ $\left.L^{2} h_{i j} / r^{2}(i, j \neq t), H=L^{2}\left(h_{x x}+h_{y y}\right) / 2 r^{2}\right)$. From the equations of motion satisfied by the fluctuations one obtains three independent second-order ODEs for the gaugeinvariant variables $Z_{1}, Z_{2}, Z_{3}$.

The QNM spectra in all three channels share a characteristic feature: an infinite sequence of (asymptotically) equidistant frequencies approximated (for $q=0$ ) by a simple formula $99,100,107$

$$
\omega_{n}=2 \pi T n( \pm 1-i)+\omega_{0}, \quad n \rightarrow \infty .
$$

Each frequency has a non-trivial dependence on $q$ [99, 100]. Spectra in the shear and sound channels are shown in Fig. (14). In addition to the sequence mentioned above, they contain the so called hydrodynamic frequencies shown in Fig. 14 by hollow red dots. The hydrodynamic frequencies are remarkable, in that their existence and dependence on $q$ are predicted by the hydrodynamics of the dual field theory. For example, low-frequency, small momenta fluctuations of the stress-energy tensor $T_{\mu \nu}$ of any $d$-dimensional theory are characterized by the dispersion relations (see e.g. [15, 110, 416])

$$
\begin{aligned}
& \omega=-i \frac{\eta}{\epsilon+P} q^{2}+O\left(q^{4}\right) \\
& \omega= \pm c_{s} q-\frac{i}{\epsilon+P}\left[\frac{\zeta}{2}+\left(1-\frac{1}{d}\right) \eta\right] q^{2}+O\left(q^{3}\right)
\end{aligned}
$$

where $\epsilon, P, \eta, \zeta$ are, respectively, energy density, pressure, shear and bulk viscosities, and $c_{s}$ is the speed of sound. For conformal theories, some of the higher order terms are also known (see e.g. [416]). In quantum field theory, the dispersion relations (139), (140) appear as poles of the retarded correlation functions of the stress-energy 
tensor. Therefore, according to the holographic dictionary discussed in Section 8.2 Eqs. (139), (140) are precisely the lowest quasinormal frequencies in the spectrum of gravitational perturbations. For $\mathcal{N}=4 \mathrm{SYM}$ in $d=4$ with zero chemical potential we have $\zeta=0, c_{s}=1 / \sqrt{3}, \epsilon+P=s T$, where $s$ is the (volume) entropy density, and the dispersion relations (139), (140) become

$$
\begin{aligned}
& \omega=-i \frac{\eta}{s T} q^{2}+O\left(q^{4}\right), \\
& \omega= \pm \frac{q}{\sqrt{3}}-\frac{i \eta}{s T} q^{2}+O\left(q^{3}\right) .
\end{aligned}
$$

On the other hand, the lowest QNMs of the fluctuations $Z_{1}, Z_{2}$ of the dual black brane background (125) can be computed analytically [415, 413]:

$$
\begin{aligned}
& \omega=-i \frac{1}{4 \pi T} q^{2}+O\left(q^{4}\right), \\
& \omega= \pm \frac{q}{\sqrt{3}}-\frac{i}{4 \pi T} q^{2}+O\left(q^{3}\right) .
\end{aligned}
$$

Comparing Eqs. (141) and (143), (142) and (144), one finds that 1) the real part of the mode (144) is correctly predicted by hydrodynamics (yet another piece of evidence in favor of the AdS/CFT conjecture) and 2) assuming the validity of AdS/CFT, the ratio $\eta / s$ is equal to $1 / 4 \pi$ in $\mathcal{N}=4 \mathrm{SYM}$ theory (in the limit of infinite coupling and infinite $N_{c}$, where the dual gravity description is valid).
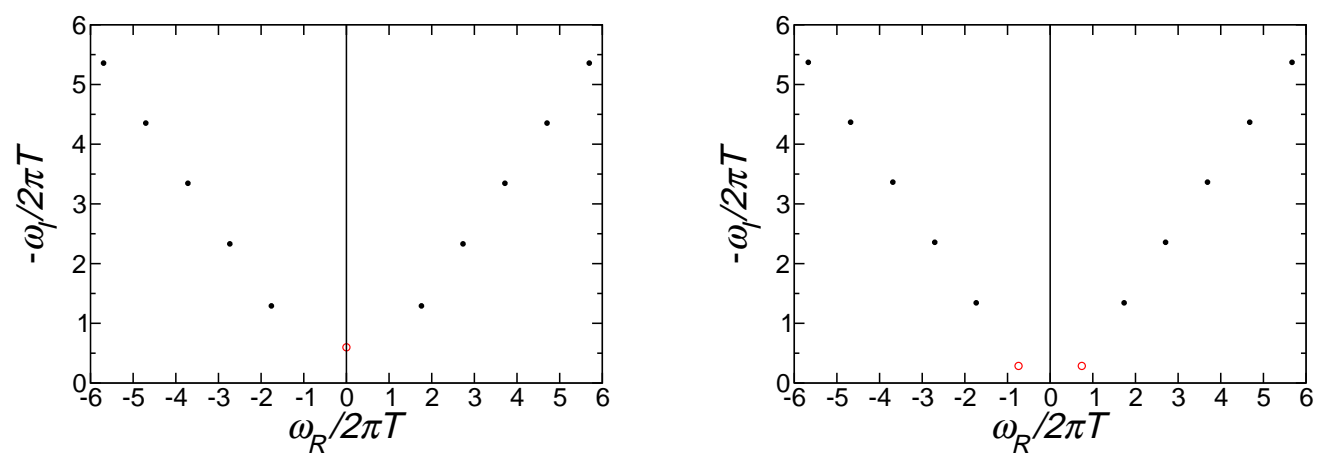

Figure 14. Quasinormal spectrum of black three-brane gravitational fluctuations in the "shear" (left) and "sound" (right) channels, shown in the plane of complex frequency $\mathfrak{w}=\omega / 2 \pi T$, for fixed spatial momentum $\mathfrak{q}=q / 2 \pi T=1$. Hydrodynamic frequencies are marked with hollow red dots (adapted from [109]).

This simple example illustrates a general method of extracting physical quantities of strongly coupled quantum field theories from the QNM spectra of their gravity duals. Bulk fluctuations corresponding to operators of conserved currents in the dual field theory in Minkowski spacetime are guaranteed to have hydrodynamic frequencies in their QNM spectra. All such frequencies have the generic property that $\omega \rightarrow 0$ for $q \rightarrow 0$, characteristic of long wavelength, small frequency fluctuations in flat space. For $\mathcal{N}=4 \mathrm{SYM}$, such operators are (in addition to $T_{\mu \nu}$ ) the $R$-current and the supercurrent. Their corresponding bulk fluctuations are, respectively, the $U(1)$ and 
the Rarita-Schwinger fluctuations in the background (125). The hydrodynamic QNMs of these fluctuations were first computed in Refs. [415, 100, 417. The full QNM spectrum of electromagnetic/gravitational fluctuations in the background (125) was computed in Refs. [100, 109]. The full QNM spectrum of the Rarita-Schwinger field has not yet been determined. Note that for theories in a finite volume (dual to black holes rather than branes) hydrodynamic QNMs, strictly speaking, do not exist as the momentum $q$ is discrete. However, for the large-radius asymptotically AdS BHs, the emergence of the hydrodynamic behavior in the limit $r_{+} / L \rightarrow \infty$ is easily detected (see e.g. Eq. (109)).

The interpretation of non-hydrodynamic QNMs is obscured by the fact that very little is known about thermal correlation functions at strong coupling. Typical singularities of such correlators at weak coupling appear to be cuts rather than poles (this problem is discussed in Ref. 418]). Poles associated with the QNM sequence (138), for example, cannot be interpreted as quasiparticles of $\mathcal{N}=4 \mathrm{SYM}$, since their imaginary part is large.

For backgrounds more complicated than the one of the black brane (125), the gauge-invariant variables $Z_{i}$ may involve fields other than metric fluctuations. Generically, these variables form a system of coupled ODEs (simple examples can be found in 110, 111, 410).

A separate problem is the computation of the corrections to QNM spectra coming from higher-derivative terms in the relevant supergravity actions. In the dual field theory such corrections correspond, in particular, to coupling constant corrections to transport coefficients. For instance, higher-derivative corrections to the type IIB supergravity action result in 't Hooft coupling corrections to the shear viscosityentropy density ratio in $\mathcal{N}=4$ SYM [419, 420, 421]

$$
\frac{\eta}{s}=\frac{1}{4 \pi}\left(1+\frac{15 \zeta(3)}{\lambda^{3 / 2}}+\cdots\right), \quad \lambda \gg 1,
$$

where $\zeta(3) \approx 1.202$ is Apéry's constant. Corrections to other transport coefficients have also been considered [422, 423, 424, 425].

\subsection{Universality of the shear mode and other developments}

The existence of "hydrodynamic" QNMs is a generic feature of black branes: these QNMs must appear in the spectra of fluctuations dual to conserved currents in translationally invariant backgrounds. In some cases, the lowest QNMs can be computed analytically even for rather general metrics. For example, for a black $p$ brane metric of the form

$$
d s^{2}=g_{t t}(r) d t^{2}+g_{r r}(r) d r^{2}+g_{x} x(r) \sum_{i=1}^{p}\left(d x^{i}\right)^{2},
$$

with all other background fields vanishing, one finds that the component of an electric field fluctuation parallel to the brane possesses a "hydrodynamic" frequency

$$
\omega=-i D q^{2}+O\left(q^{4}\right)
$$

where the coefficient $D$ is given in terms of the metric components:

$$
D=\frac{\sqrt{-g\left(r_{H}\right)}}{g_{x x}\left(r_{H}\right) \sqrt{-g_{t t}\left(r_{H}\right) g_{r r}\left(r_{H}\right)}} \int_{r_{H}}^{\infty} d r \frac{-g_{t t}(r) g_{r r}(r)}{\sqrt{-g(r)}}
$$


and $r_{H}$ is the position of the horizon. If there exists a quantum field theory (QFT) dual to this background, the electromagnetic fluctuation naturally couples to a conserved $U(1)$ current in the theory, and $D$ is interpreted as a diffusion constant of a corresponding $U(1)$ charge.

Similarly, the lowest gravitational QNM in the shear channel (135) for the metric (146) can be computed analytically [114. The result is an expression similar to Eqs. (147)-(148), but it can be further simplified using either Buchel-Liu's theorem [426] or the alternative proof given in [7]. Surprisingly, it turns out that this mode is universal: its frequency is given by Eq. (143) for any background with a metric of the form (146). (It is important to note that the shear mode fluctuation decouples from the fluctuations of other fields 427.)

The universality of the gravitational quasinormal shear mode has an important consequence for a dual QFT: the ratio of shear viscosity to entropy density has a universal value $\eta / s=1 / 4 \pi$ for all theories with gravity duals, in the limit where the gravity dual description is valid (e.g., in the limit of infinite $N_{c}$ and infinite 't Hooft coupling for $\mathcal{N}=4 S U\left(N_{c}\right)$ SYM in four dimensions). Earlier proofs of the shear viscosity universality not involving QNMs were given in 427, 428. Charged black brane backgrounds were considered in [410, 429, 430, 431, 432, 433, 434, 435, 424].

The viscosity bound conjecture 436. prompted further investigation of the black brane QNM spectrum in higher-derivative gravity [437, 438, 439, 440, 441, 442, 443, 444, 445]. The conjecture states that the ratio $\eta / s$ is bounded from below by $1 / 4 \pi$ in all physical systems. In the language of QNMs, this would mean that a correction to the $q^{2}$-coefficient in Eq. (143) coming from higher-derivative terms in the action describing gravity duals of such systems is always positive, as in Eq. (145). In some gravity models with higher-derivative terms this appears not to be the case [437, 438, 439, 440, 425]. It would be highly desirable to have a comprehensive understanding of the influence of higher-derivative terms on the shear mode and other QNMs.

QNMs of $D p$-branes and more complicated backgrounds in the holographic context were computed in 446, 447, 111, 112, 448. Time-dependent backgrounds especially relevant for modeling the behavior of the quark-gluon plasma in heavy ion collisions were investigated in [449, 316, 163]. QNMs in models with holographic mesons, Sakai-Sugimoto model and other QCD-like models were studied in [450, 451, 452, 453, 454, 455, 456, 457, 458.

The gauge-gravity duality is primarily used to investigate strongly coupled gauge theories with the help of a dual classical gravity theory. However, at least in principle, the holographic dictionary can be used to explore aspects of quantum gravity with the help of a weakly coupled gauge theory. QNMs in this and similar contexts of duality have been studied in [105, 106, 331, 336].

Recently, holographic methods were extended to include gravity backgrounds dual to non-relativistic theories as well as systems with spontaneous symmetry breaking. Exploration of QNM spectra in these models is an active area of research [37, 38].

\section{Quasinormal modes of astrophysical black holes}

In the context of the Einstein-Maxwell equations, the most general solution describing stationary axisymmetric BHs is the Kerr-Newman metric 39. For astrophysical BHs the electric charge $Q$ is likely to be negligible, being shorted out by the surrounding plasma [459, 460. Astrophysical BHs are effectively the simplest of all macroscopical 
objects, characterized only by their mass $M$ and angular momentum parameter $a$. As a consequence, their structure and their oscillation spectra are remarkably simple.

The complex frequency of a QNM yields two observables: the actual oscillation frequency and the damping time of the oscillation. For each given mode, these observables depend only on $M$ and $a$. Therefore a measurement of the frequency and damping time of a QNM can be used to infer the mass and angular momentum of the BH with potentially high accuracy [9, 79, 10, 11. Since the whole QNM spectrum depends solely on $M$ and $a$, the measurement of two or more QNM frequencies provides a stringent observational test of the no-hair theorem of general relativity [461, 462, 11, 463. The prospects for detecting the signature of $\mathrm{BH}$ oscillations in gravitational waves are the main topic of this section.

There is strong and growing observational evidence for the existence of at least two different classes of astrophysical BHs. Solar-mass BHs with $M \sim 5-20 M_{\odot}$ are usually found in X-ray binaries [464], and SMBHs with $M \sim 10^{6}-10^{9.5} M_{\odot}$ are believed to harbor most Active Galactic Nuclei (AGN) 465. At the moment there is only tentative evidence for intermediate-mass BHs (IMBHs) of mass $M \sim 10^{2}-10^{5} M_{\odot}$ 466, 467, 468. Ringdown detectability from these different classes of astrophysical BHs depends on several factors, the first of which is the sensitivity of any given gravitational wave detector. Earth-based detectors, such as LIGO and Virgo, have an optimal sensitivity band corresponding to stellar-mass BHs and IMBHs, while the planned space-based detector LISA is most sensitive to ringdowns from high-mass IMBHs and SMBHs (see Section 9.1 below). Another important factor is the extent to which QNMs are excited in astrophysical settings. The most promising scenarios to excite ringdown to a detectable level are reviewed in Section 9.2. Whatever the source of the excitation, the frequency and amplitude of ringdown waves depend on the mass and spin of the $\mathrm{BH}$. The evidence for astrophysical BHs and the present understanding of their mass and spin distributions are reviewed in Section 9.3 Measuring ringdown waves will allow us to extract interesting information, ranging from accurate measurements of the mass and spin of BHs to tests of the no-hair theorem of general relativity. These applications of gravitational wave detection are reviewed in Sections 9.6 9.8

\subsection{Physical parameters affecting ringdown detectability}

Present and planned gravitational wave detectors are located at large distance from astrophysical BHs. Therefore, for all practical purposes, a QNM as seen by a detector is well approximated by the asymptotic behavior of the wave equation at infinity, Eq. (30). We can express the waveform measured at the detector as a linear superposition of the gauge-invariant polarization amplitudes $h_{+}, h_{\times}$, where, for a given mode $(l, m, n)$,

$$
\begin{aligned}
& h_{+}=\frac{M}{r} \operatorname{Re}\left[\mathcal{A}_{l m n}^{+} e^{i\left(\omega_{l m n} t+\phi_{l m n}^{+}\right)} e^{-t / \tau_{l m n}} S_{l m n}(\iota, \beta)\right], \\
& h_{\times}=\frac{M}{r} \operatorname{Im}\left[\mathcal{A}_{l m n}^{\times} e^{i\left(\omega_{l m n} t+\phi_{l m n}^{\times}\right)} e^{-t / \tau_{l m n}} S_{l m n}(\iota, \beta)\right] .
\end{aligned}
$$

Here $\mathcal{A}_{l m n}^{+, \times}$and $\phi_{l m n}^{+, \times}$are the (real) amplitude and phase of the wave, and $S_{l m n}(\iota, \beta)$ denotes spin-weighted spheroidal harmonics of spin-weight -2 [10. The angles $(\iota, \beta)$ are adapted to the source, so that the $z$-axis is aligned with the spin of the $\mathrm{BH}$. Interferometric detectors are sensitive to the effective strain

$$
h=h_{+} F_{+}\left(\theta_{S}, \phi_{S}, \psi_{S}\right)+h_{\times} F_{\times}\left(\theta_{S}, \phi_{S}, \psi_{S}\right),
$$


where $F_{+, \times}$are pattern functions that depend on the orientation of the detector and the direction of the source (specified by the polar angles $\theta_{S}, \phi_{S}$ ) and on a polarization angle $\psi_{S}$ [153, 469]. A crucial quantity for gravitational wave detection is the signalto-noise ratio (SNR) $\rho$, defined as

$$
\rho^{2}=4 \int_{0}^{\infty} \frac{\tilde{h}^{*}(f) \tilde{h}(f)}{S_{h}(f)} d f
$$

where $\tilde{h}(f)$ is the Fourier transform of the waveform, and $S_{h}(f)$ is the noise spectral density of the detector [79]. In discussing the SNR we will usually average over source direction, detector and $\mathrm{BH}$ orientations, making use of the sky averages: $\left\langle F_{+}^{2}\right\rangle=\left\langle F_{\times}^{2}\right\rangle=1 / 5,\left\langle F_{+} F_{\times}\right\rangle=0$, and $\left\langle\left|S_{l m n}\right|^{2}\right\rangle=1 / 4 \pi$. An analysis taking into account different sky locations and orientations of the source probably requires Monte Carlo methods. At the moment, such an analysis is still lacking.

Our chances of detecting and measuring ringdown waves are mainly determined by the BH's mass $M$, by the spin parameter $a$, and by the ringdown efficiency $\epsilon_{\mathrm{rd}}$. The latter quantity is defined as the fraction of the total mass-energy of the system radiated in ringdown waves, and it is well approximated by [10, 89.

$$
\epsilon_{\mathrm{rd}} \approx \frac{Q_{l m n} M \omega_{l m n}}{32 \pi}\left[\left(\mathcal{A}_{l m n}^{+}\right)^{2}+\left(\mathcal{A}_{l m n}^{\times}\right)^{2}\right] .
$$

These three parameters (mass, spin and efficiency) affect the detectability and measurability of the signal in different ways.

The BH mass sets the frequency scale and damping time of the emitted radiation. For a Schwarzschild $\mathrm{BH}$, the fundamental QNM with $l=2$ (that dominates the radiation in most cases [89]) has frequency $f$ and damping time $\tau$ given by

$$
\begin{aligned}
& f=1.207 \cdot 10^{-2}\left(10^{6} M_{\odot} / M\right) \mathrm{Hz}, \\
& \tau=55.37\left(M / 10^{6} M_{\odot}\right) \mathrm{s} .
\end{aligned}
$$

Earth-based detectors are limited at low frequency by a seismic cutoff $f_{s}$ (a plausible estimate for second-generation detectors being $f_{s} \sim 10 \mathrm{~Hz}$ for the Einstein Gravitational Wave Telescope, and $f_{s} \sim 20 \mathrm{~Hz}$ for Advanced LIGO). Therefore they can detect the fundamental $l=m=2$ QNM as long as the BH mass $M \lesssim 1.2 \times 10^{4}\left(\mathrm{~Hz} / f_{s}\right) M_{\odot}$ if the $\mathrm{BH}$ is non rotating, and $M \lesssim 2.7 \times 10^{4}\left(\mathrm{~Hz} / f_{s}\right) M_{\odot}$ if the BH is rotating near the Kerr limit (see Table I in Ref. [11). LISA is limited at high masses (low frequencies) by acceleration noise, and at low masses by the condition that the damping time $\tau$ should be longer than the light-travel time $T_{\text {light }} \simeq 16.7 \mathrm{~s}$ corresponding to the planned armlength $\left(L \simeq 5 \cdot 10^{9} \mathrm{~m}\right)$. Thus LISA can detect ringdown waves from $\mathrm{BHs}$ in the range $10^{5} M_{\odot} \lesssim M \lesssim 10^{9} M_{\odot}$ [10]. To summarize: Earth-based detectors are sensitive to the ringdown of stellar-mass $\mathrm{BHs}$ and of relatively low-mass IMBHs, and LISA can observe mergers of IMBHs and SMBHs throughout the whole universe. There is a chance that the ongoing ringdown searches in data from Earth-based gravitational wave detectors [12, 470, 471, 472, 473, 474, 13 . may provide the first incontrovertible evidence of the existence of IMBHs.

As discussed in Section 5.3, the BH spin (for a given BH mass) determines all frequencies of the Kerr QNM spectrum. For QNMs with $m>0$ the quality factor increases with spin (see Fig. 9). Since the detectability of a gravitational wave signal by matched filtering scales with the square root of the number of cycles, highly spinning $\mathrm{BHs}$ could be the best candidates for detection [89. However, exciting QNMs of fastrotating $\mathrm{BHs}$ seems to be harder, as the excitation factors tends to zero as $a / M \rightarrow 1$ 
(see [70, 167] and Section 3.2). In hindsight, this is not surprising: the build-up of energy in a long-lived resonant mode usually takes place on a timescale similar to the eventual mode damping, so it should be difficult to excite a QNM with characteristic damping several times longer than the dynamical timescale of the excitation process 263. Numerical simulations of the merger of comparable-mass BH binaries suggest that QNM excitation is mildly dependent on the initial spin of the components (see 475 and Section 9.2), but further investigation is required to clarify this issue.

There are different ways of quantifying the excitation of QNMs by generic initial data [77, 173, 166, 3, 167. One can operationally define a ringdown efficiency $\epsilon_{\mathrm{rd}}$, which is directly related to the gravitational wave amplitude as illustrated by Eq. (153), and hence to the SNR of the signal for a given detector [89, 10]. Different empirical notions for the "ringdown starting time" (which is intrinsically ill-defined [169]) yield very different ringdown efficiencies [476, 44, 477]. From the point of view of detection, a suitable definition of the ringdown starting time is the one proposed by Nollert, using an "energy-maximized orthogonal projection" of a given numerical waveform onto QNMs 44, 11. The relative excitation of different modes is even harder to determine than the overall ringdown efficiency, but it is particularly relevant for tests of the no-hair theorem using ringdown waves [461, 10, 11. Ref. 28, discusses this issue in a general context, and Berti et al. discuss this issue in a general context [28, and give preliminary estimates of the excitation of different multipoles in binary $\mathrm{BH}$ mergers 44, 475].

In Section 9.2 we review promising astrophysical scenarios that could produce detectable ringdown signals (i.e., large efficiencies): accretion, stellar collapse leading to $\mathrm{BH}$ formation and compact object mergers. In Section 9.3 we summarize the current theoretical and experimental understanding of $\mathrm{BH}$ masses and spins.

\subsection{Excitation of black hole ringdown in astrophysical settings}

In principle, most dynamical processes involving BHs excite QNMs to some degree. For the purpose of gravitational wave detection from astrophysical BHs, the question is not whether QNMs are excited, but whether they are excited to a detectable level. $\mathrm{BH}$ QNMs can be excited in a variety of astrophysical settings, such as accretion, collapse and compact binary mergers. As we will see, analytical estimates and numerical calculations show that the most promising source of detectable ringdown waves is the merger of two compact objects leading to $\mathrm{BH}$ formation.

Ringdown excitation by accretion An early study highlighting the importance of QNM ringing is the classic analysis of the gravitational radiation emitted by particles falling radially into a Schwarzschild $\mathrm{BH}$ 43. Unlike stellar oscillation modes (which play an important role in the orbital dynamics of compact binaries [478, 479, 480, 481) $\mathrm{BH}$ QNMs are hard to excite by matter orbiting around the $\mathrm{BH}$. The reason is that weakly damped QNMs are associated with unstable geodesics at the light ring (see 208. and references therein), and for Kerr BHs the light-ring frequency is always larger than the frequency of the innermost stable circular orbit (ISCO), as illustrated in the left panel of Fig. 15. Higher overtones may have lower frequencies, but they are harder to excite because their quality factor is too small. According to this intuitive description, QNM excitation requires the accreting matter to cross the light ring. Indeed, QNMs are always excited by particles falling along generic geodesics into Kerr BHs (see 23] and Appendix C of [4] for comprehensive lists of references). Lumps of 

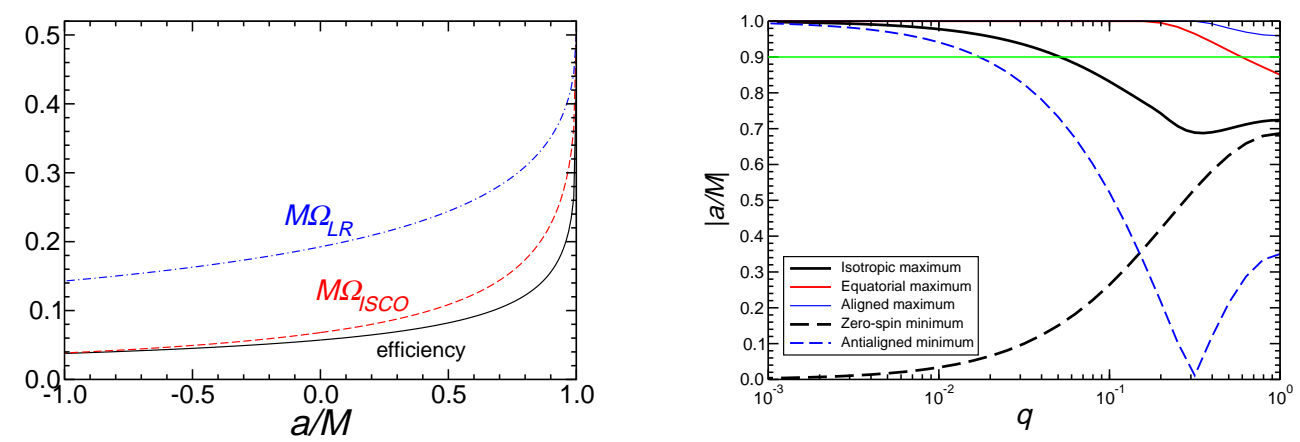

Figure 15. Left: ISCO frequency, light-ring frequency and radiation efficiency for Kerr BHs (positive values of $a / M$ refer to corotating orbits, negative to counterrotating orbits). Right: bounds on the final spin expected to result from a binary BH merger as a function of the binary's mass ratio $q$ (from Ref. [482]).

matter accreting onto a $\mathrm{BH}$ at appropriate rates could potentially excite QNMs to a detectable amplitude. Unfortunately, even the most optimistic estimates suggest that the wave amplitude is too small 266. For generic accretion flows, simple analytical arguments 28 show that destructive interference reduces the ringdown amplitude [23, 483, 484]. This conclusion is confirmed by numerical simulations of neutron star (NS)-BH mergers: if the NS is tidally disrupted well before merger, accretion of the NS material onto the $\mathrm{BH}$ proceeds incoherently, and the ringdown signal is replaced by an abrupt cutoff in Fourier space at the tidal disruption frequency (see Section 9.2 and Ref. [485). If it occurs in nature, a highly intermittent hyperaccretion scenario [486] may excite QNM ringing in a nearly resonant fashion for large spin parameters, and potentially lead to ringdown radiation strong enough to be detectable by Advanced LIGO.

Collapse to a black hole There are several excellent reviews on gravitational wave signatures from supernova core collapse and on the underlying physics [23, 487, 488, 489, 490. When the core of a massive star collapses, it produces a nonspherical protoneutron star. Depending on details of the supernova explosion, the protoneutron star may collapse to a $\mathrm{BH}$, emitting a burst of gravitational waves due to the rapidly shriking mass-quadrupole moment of the protoneutron star and to the QNM ringing of the nascent $\mathrm{BH}$.

Perturbative calculations of gravitational wave emission from rotating gravitational collapse to a BH were first carried out in the 1970s by Cunningham, Price and Moncrief 63, 64, 65, improved upon by Seidel and collaborators [491, 492, 493, 494, and more recently by Harada, Iguchi and Shibata [495. These studies suggest that gravitational waves are mainly generated in the region where the Zerilli potential is large, and that the signal is usually dominated by QNM ringing of the finally formed BH (see [495] for exceptions). Simplified simulations based on a free-fall (Oppenheimer-Snyder) collapse model yield a small energy output, with a typical core collapse radiating up to $\simeq 10^{-7} M$ in gravitational waves. Most of the radiation 
is quadrupolar $(l=2)$, radiation in $l=3$ being typically two to three orders of magnitude smaller (see Fig. 9 in 63]). Recent perturbative studies suggest that magnetic fields could increase the energy output by several orders of magnitude [496, 497].

The first numerical simulation of collapse in two dimensions was carried out by Nakamura 498, but numerical problems prevented gravitational radiation extraction. For a long time the seminal 1985 work of Stark and Piran 71 has been the only nonperturbative, axisymmetric calculation of gravitational wave emission from stellar collapse. The waveform resembles that emitted by a point particle falling into a $\mathrm{BH}$, but with a reduced amplitude. The total energy emitted increases with the rotation rate, ranging from $\sim 10^{-8} M$ for $a / M=0$ to $\sim 7 \times 10^{-4} M$ as $a / M \rightarrow 1$. Rotational effects halt the collapse for some critical value of $(a / M)_{\text {crit }}$ which is very close to unity, and depends on the artificial pressure reduction used to trigger the collapse. Stark and Piran find that the energy emitted $E / M \simeq 1.4 \times 10^{-3}(a / M)^{4}$ for $0<a / M<(a / M)_{\text {crit }}$, and for larger spins it saturates to a maximum value $\epsilon_{\max } \sim 10^{-4}$. Note however that the maximum energy radiated is very sensitive to the amount of artificial pressure reduction used to trigger the collapse: the $99 \%$ pressure reduction used in the simulations of Stark and Piran essentially produced a free-fall collapse, presumably overestimating the radiation efficiency.

This calculation has recently been improved using a three-dimensional code [49, 500, 72, 501]. Ref. 500] choose as the initial configuration the most rapidly rotating, dynamically unstable model described by a polytropic equation of state (EOS) with $\Gamma=2$ and $K=100$, having a dimensionless rotation rate $\simeq 0.54$, and trigger collapse by reducing the pressure by $\lesssim 2 \%$. The "+" polarization is essentially a superposition of modes with $l=2$ and $l=4$, and the " $\times$ " polarization is a superposition of modes with $l=3$ and $l=5$. The energy lost to gravitational waves according to these simulations is at most $\simeq 1.45 \times 10^{-6}\left(M / M_{\odot}\right)$, two orders of magnitude smaller than the estimate by Stark and Piran for the same value of the angular momentum, but larger than the energy losses found in recent calculations of rotating stellar core collapse to protoneutron stars [502. Ref. 501] confirms the basic scaling $E / M \approx(a / M)^{4}$ for $a / M \lesssim 0.54$ (the largest rotation rates yielding equilibrium models in uniform rotation), but at variance with Stark and Piran's simulations it finds that the efficiency has a local maximum for large rotation rates. If the collapse is triggered only by pressure depletion, the overall efficiency for uniformly rotating models is quite low $\left(E / M \approx 10^{-7}-10^{-6}\right)$, the mass quadrupole does not change very rapidly, and higher multipoles are not significantly excited. However the total energy radiated can increase by up to two orders of magnitude if velocity perturbations are present in the collapsing star. A rapidly rotating polytropic star at $10 \mathrm{kpc}$ can produce maximum characteristic amplitudes $h_{c} \approx 5 \times 10^{-22}\left(M / M_{\odot}\right)$ at characteristic frequencies ranging between $f_{c} \approx 500 \mathrm{~Hz}$ and $f_{c} \approx 900 \mathrm{~Hz}$, and could be detectable with SNRs as large as about 30 by Earth-based interferometers.

In conclusion, the ringdown efficiency is very sensitive to poorly known details of the mechanism triggering the collapse. Simulations with more realistic microphysics are required to study the complex signal preceding the ringdown phase, as well as details of QNM excitation by matter accreting onto the newly formed $\mathrm{BH}$.

Mergers of compact objects leading to black hole formation Numerical relativity simulations of compact binary mergers made enormous progress since 1999, when the two classic reviews on QNMs [4, 5] were written. This section is an attempt to summarize aspects of this progress of interest for the detection of ringdown waves from 
astrophysical BHs.

Since NSs cannot have masses larger than about $3 M_{\odot}$, Eq. (84) implies that NS-NS mergers are potentially relevant only for the detection of $\mathrm{BH}$ ringdowns by Earth-based interferometers, such as LIGO and Virgo. On the other hand, NS-BH and $\mathrm{BH}-\mathrm{BH}$ mergers are plausible targets for ringdown detection by both Earth-based and future space-based detectors, so we will discuss them in more detail.

(1) NS-NS mergers - In 1999 Shibata and collaborators carried out the first successful equal-mass NS-NS merger simulation for a polytropic index $\Gamma=2$ 503, 93. The first reasonably accurate calculation of gravitational waveforms was possible a few years later, in 2002 [504]. Unequal-mass binaries with a $\Gamma=2$ polytropic EOS were studied in [505], where it was found that a $\mathrm{BH}$ forms when the total rest-mass of the system is larger than $\sim 1.7$ times the maximum allowed rest-mass of spherical NSs, irrespective of mass ratio (which however affects significantly the waveforms and the mass of the disk forming around the newly born BH). More realistic EOSs and larger parameter spaces were considered in Refs. [506, 507]. Ref. [507] uses stiff EOSs and models binary NSs of ADM mass $M \gtrsim 2.6 M_{\odot}$. For all mass ratios $0.65 \lesssim q \leq 1$, a $\mathrm{BH}$ forms whenever the mass $M>M_{\mathrm{th}}$. The threshold value $M_{\mathrm{th}} \simeq 1.3-1.35 M_{\max }$ (where $M_{\max }$ is the maximum mass allowed by the given EOS for cold, spherical NSs) depends on the EOS. If $M<M_{\text {th }}$ the merger results in a hypermassive NS of large ellipticity, emitting quasiperiodic gravitational waves at frequencies $\sim 3-4 \mathrm{kHz}$ for $\lesssim 100 \mathrm{~ms}$. After this phase the NS may or may not collapse to a BH. For total binary masses in the range $M \approx 2.7-2.9 M_{\odot}$, the $l=m=2$ ringdown frequency emitted in the collapse to a $\mathrm{BH}$ is $\approx 6.5-7 \mathrm{kHz}$, with amplitude $\sim 10^{-22}$ at a distance of $50 \mathrm{Mpc}$. Therefore BH ringdown from NS-NS mergers is unlikely to be detected: the amplitude is too low and (most importantly) the frequency is too high for present and planned Earth-based gravitational wave detectors. It is interesting to note that NS-NS merger simulations typically lead to final $\mathrm{BH}$ spins $a / M \approx 0.8$ [507, 508, not very different from the value $a / M \simeq 0.69$ predicted by equal-mass $\mathrm{BH}$ merger simulations. Various groups have recently carried out NS-NS merger simulations with magnetic fields, finding that aligned poloidal fields can delay the merger and strongly affect the gravitational wave signal [509, 510, 511].

(2) NS-BH mergers - NS-BH binaries are potentially among the most interesting $\mathrm{BH}$ ringdown sources. From Eq. (154), the QNM frequency for non-spinning BHs with $M \gtrsim 10 M_{\odot}$ is within the sensitivity window of LIGO and Virgo. Theory and observations [512, 464, 513] suggest that binaries containing BHs with masses in this range should be common. Furthermore, high mass-ratio systems do not tidally strip the NS, producing a merger with a clean ringdown signal. The simulations described in the rest of this section suggest that NS-BH mergers are promising sources of ringdown waves and (when the $\mathrm{BH}$ is spinning) excellent candidates as central engines for gamma-ray bursts (GRBs).

NS-BH merger simulations became possible once the moving puncture approach [15, 16] proved successful in evolving BH-BH binaries. Shibata and Uryu [514, 515, first evolved two binaries consisting of $(1)$ a non-rotating $\mathrm{BH}$ of mass $\approx 3.2 M_{\odot}$ or $\approx 4 M_{\odot}$, respectively, and $(2)$ a NS of rest mass $\approx 1.4 M_{\odot}$, modeled by a $\Gamma$-law EOS with $\Gamma=2$. A larger set of simulations with non-spinning BHs of masses $M \sim 3.3-4.6 M_{\odot}$ and NSs of mass $M \sim 1.4 M_{\odot}$ was performed in Ref. [516. The NS is tidally disrupted, and the system results in a $\mathrm{BH}$ with spin $a / M \approx 0.5-0.6$. Non-spinning NS-BH mergers can only produce massive tori and fuel short-hard GRBs if the NS has compactness $M / R \lesssim 0.145$. Furthermore, when tidal disruption occurs the QNM amplitude quickly 
decreases, because the incoherent accretion of material is ineffective at resonantly exciting the mode (see Fig. 6 of Ref. [516).

The first code capable of handling NS-NS, NS-BH and BH-BH binary evolutions comprising more than 4 orbits was recently described in Ref. 508. Gravitational waveforms from NS-BH mergers were presented in Ref. [485] and classified into three classes as follows: 1) For small mass ratio $q$ and small NS compactness (e.g., if $q \lesssim 3$ for $M / R=0.145$ and $\Gamma=2$ ) tidal disruption occurs outside the ISCO, and there is no QNM excitation; 2) For some systems, mass shedding occurs before the binary reaches the ISCO. Most of the NS is swallowed by the BH before tidal disruption is completed and QNMs are excited, but only to a low amplitude; 3) If tidal effects do not play an important role the waveforms shows significant QNM excitation, as they always do for BH-BH mergers. The latter class of NS-BH mergers is clearly the most promising for $\mathrm{BH}$ ringdown detection. Whenever ringdown is not significantly excited, kicks are also suppressed: this confirms the crucial role played by the merger/ringdown phase in determining the magnitude of the kick resulting from compact binary mergers (see Section 9.8 below). The results of Ref. [485] are in good agreement with NS-BH codes developed by other groups [517, 518, 519]. Ref. [517] deals with systems where the NS is irrotational, the $\mathrm{BH}$ is non-rotating and the mass ratio $q=1 / 3$. These simulations lead to the formation of BHs with $a / M \approx 0.5-0.8$. Most of the NS material is prompty accreted, and no more than $3 \%$ of the NS mass is ejected into a gravitationally bound disk. This disk mass, while larger than the typical values found in Ref. [485, is probably not enough to trigger short-hard GRBs.

Results from non-spinning NS-BH mergers show that the disk mass is typically too low to fuel GRBs. For example, Fig. 7 of Ref. 485] shows that, if the BH is non-spinning, the formation of a disk requires a "fat" NS with radius $R \gtrsim 14 \mathrm{~km}$. The few simulations presently allowing for (aligned or anti-aligned) BH spins have a drastically different outcome, indicating that spin plays a crucial role in fueling GRBs 518. In these simulations the number of orbits before merger increases as the spin is varied between $a / M=-0.5$ (anti-aligned), 0.0 and 0.75 (for fixed mass ratio $q \simeq 1 / 3$ ). In the latter case the final $\mathrm{BH}$ spin is $a / M \simeq 0.88$, and the tidal disruption of the NS leads to the formation of a massive disk of about $0.2 M_{\odot}$, potentially capable of driving GRBs. The production of GRBs by NS-BH mergers and the possibility to detect the final spin orientation using observations of the merger/ringdown phase are promising areas of research for multi-messenger astronomy using a network of ground-based detectors in conjunction with traditional electromagnetic observations (see e.g. [520, 521, 522, 523, 524]).

(3) $\mathrm{BH}$-BH mergers - After 40 years of developments in numerical relativity, recent breakthroughs [14, 15, 16, finally allowed simulations of the merger and ringdown of $\mathrm{BH}$ binaries. Extensive collaborations to use numerical merger waveforms in gravitational wave searches have just started [525]. A discussion of the accuracy of numerical simulations and of their rapid progress in the last few years would take us too far (see Refs. 14, 526] for reviews). From the point of view of this review, the main result of these merger simulations is that QNM ringing is observed in all binary BH merger simulations. This is quite unlike NS-BH and NS-NS mergers, where tidal effects can sometimes suppress ringdown excitation because of the incoherent accretion of material onto the newly formed $\mathrm{BH}$. Another important difference is that the gravitational waveform from a $\mathrm{BH}-\mathrm{BH}$ merger depends on the total mass of the system via a trivial rescaling, so $\mathrm{BH}-\mathrm{BH}$ systems are interesting for ringdown detection by both Earth-based and space-based detectors. 
The mass-ratio dependence of the ringdown efficiency can be estimated by simple arguments [89, 44]. The quadrupole moment of a body of mass $M$ with a "bump" of mass $\mu \ll M$ is $Q \sim \mu M^{2}$. The oscillation frequency of the system $f \sim 1 / M$; hence, the radiated power $d E / d t \sim\left(d^{3} Q / d t^{3}\right)^{2} \sim\left(f^{3} Q\right)^{2} \sim \mu^{2} / M^{2}$. For a binary with mass ratio $\mu / M$ the inspiral lasts $\sim(M / \mu)$ cycles times the orbital time scale $T \sim M$, so the total energy loss during the inspiral is $E_{\text {insp }} \sim(M / \mu)\left(\mu^{2} / M^{2}\right) M \sim \mu$. By contrast, a typical ringdown waveform lasts only a few cycles, so the ringdown energy loss $E_{\text {ringdown }} \sim M\left(\mu^{2} / M^{2}\right) \sim \mu^{2} / M$ (compare with the classic result for infalling particles in Ref. 43]) and $E_{\text {ringdown }} / E_{\text {insp }} \sim \mu / M$ : ringdown is negligible with respect to inspiral for extreme-mass ratio binaries. If we naively extrapolate these estimates to bodies of comparable masses (interpreting $M$ as the total mass of the binary and $\mu \rightarrow m_{1} m_{2} / M$ as the reduced mass) we find that $E_{\text {ringdown }} / M \sim \eta^{2}$, where $\eta \equiv \mu / M$ is the so-called symmetric mass ratio $(\eta \rightarrow 1 / 4$ in the comparable-mass limit).

Physical arguments to estimate the prefactors suggest that the merger/ringdown waveform should actually dominate over inspiral for binaries with mass ratio $q \gtrsim 1 / 10$ [89, 10, and numerical simulations of quasicircular inspirals of comparable-mass mergers have borne out this expectation. For non-spinning binary BH mergers, the fraction of energy radiated $\left(M-M_{\mathrm{fin}}\right) / M$ (where $M$ denotes the total mass of two BHs in isolation and $M_{\text {fin }}$ is the mass of the final BH), as well as the final spin $a / M$, have been extensively studied [477, 44, 527, 528, 528. Ref. 527] fits data from the simulations by the Goddard group by a relation of the form

$$
\begin{aligned}
& M_{\mathrm{fin}} / M=1+(\sqrt{8 / 9}-1) \eta-0.498( \pm 0.027) \eta^{2}, \\
& a / M_{\mathrm{fin}}=\sqrt{12} \eta-2.900( \pm 0.065) \eta^{2} .
\end{aligned}
$$

This result is consistent with the fitting formula given in 44 using a different normalization. Frequencies and damping times of different multipolar components can be estimated using either a standard least-squares algorithm [477] or Prony methods, which are in many ways optimal to estimate the parameters of damped exponentials in noise [529]. By monitoring the frequencies and damping times after merger we can monitor inaccuracies in the higher multipolar components of numerical simulations, and possibly explore non-linear effects (see e.g. Section IV in Ref. [44]).

Quantifying the fraction of energy radiated in ringdown is inherently ambiguous. The reason for this difficulty can essentially be traced back to the non-completeness of QNMs [169, 2, 3, 44]. An operational viewpoint to isolate the ringdown contribution is given by Nollert's energy-maximized orthogonal projection (EMOP) criterion [169, 44. The idea is to determine the starting time of a ringdown waveform by assuming that the frequency of the ringdown waveform is known, and performing matchedfiltering (in white noise) of the numerical waveform, using a damped sinusoid as the "detection template". Since ringdown is essentially monochromatic, this should give a reasonably good, frequency-independent and detector-independent estimate of the fraction of energy that we can expect to detect by a ringdown search. For more details on ringdown search techniques, see Section 9.4 below.

An application of the EMOP criterion to numerical simulations shows that ringdown typically contributes $\sim 42 \%$ of the energy radiated by the last two cycles of BH-BH mergers with mass ratio $q \geq 1 / 4$ (see Tables VI and VII of Ref. [44]). By combining Prony methods for estimating frequencies and the EMOP approach to estimate the ringdown starting time, we find the following estimates for the energy emitted in ringdown as a result of the merger of non-spinning, quasi-circular $\mathrm{BH}$ 
binaries 44

$$
\frac{E_{\text {ringdown }}^{l=2}}{M} \simeq 0.271 \frac{q^{2}}{(1+q)^{4}}, \quad \frac{E_{\text {ringdown }}^{l=3}}{M} \simeq 0.104 \frac{q^{2}(q-1)^{2}}{(1+q)^{6}} .
$$

For non-spinning, equal-mass binaries, the merger/ringdown signal as the system's orbital angular momentum is reduced (so a quasicircular merger slowly turns into an head-on collision) has been studied in Refs. 530, 531. In the head-on limit one gets a radiated energy $\sim 0.1 \% M$. In the intermediate regime, where two equal-mass $\mathrm{BHs}$ merge along orbits with large residual eccentricity, the radiated energy decays (roughly) exponentially (see Table I and Fig. 7 in Ref. 530]) and the final BH spin has a local maximum $j_{\text {fin }} \simeq 0.724 \pm 0.13$ [530, 531]. For preliminary studies of the ringdown efficiency in the merger of quasicircular, spinning binaries, see Refs. [475, 532, 533.

Predictions of the spin of a $\mathrm{BH}$ resulting from a merger are very interesting from the point of view of ringdown detection. For example, if we measure the masses and spins during the inspiral we may be able to predict the final spin and reduce the errors in parameter estimation [534, 535]. For a summary of semianalytic models and fitting formulas to predict the final spin from generic mergers, we refer the reader to 536, 537, 538, 539. Some insight into the general outcome of a spinning merger can be obtained by looking at the right panel of Fig. 15. For simplicity, consider three different merger scenarios: i) in the isotropic scenario, both BH spins are distributed isotropically; ii) in the aligned spin scenario, the individual $\mathrm{BH}$ spins in the binary are assumed to be aligned (for example, in "wet mergers" the alignment could be caused by torques from accreting gas, as suggested in [540]); iii) in the equatorial merger scenario, the smaller $\mathrm{BH}$ is supposed to orbit in the equatorial plane of the larger hole (e.g. because of Newtonian dynamical friction in a flattened system), but the spin orientation of the smaller $\mathrm{BH}$ is distributed isotropically. The right panel of Fig. 15 shows the maximum and minimum spin resulting from a merger in these three scenarios. These curves are obtained by (1) fixing some value of the mass ratio $q,(2)$ averaging over angles according to the three different assumptions listed above, and (3) maximizing or minimizing the final average spin resulting from a merger (where the average is computed using the fitting formulas of Ref. [537]) in the $\left(\left|\mathrm{j}_{1}\right|,\left|\mathrm{j}_{2}\right|\right)$ plane, where $\left|\mathrm{j}_{i}\right|$ is the spin magnitude of $\mathrm{BH} i=1$, 2. Not surprisingly, the minimum average final spin always corresponds to the case where both BHs are non-spinning (dashed black line). The maximum average spin in the three cases is shown by the continuous black (isotropic), red (equatorial) and blue (aligned) lines. The dashed blue line shows the (modulus of) the minimum spin that could be achieved if we allow for antialignment of both spins with respect to the orbital angular momentum (a spin flip becomes possible when the mass ratio $q \approx 1 / 3$ ). The most interesting prediction of this plot is the existence of a narrow funnel between the solid black and dashed black lines: on average, isotropic major mergers (with $q \gtrsim 0.2$ or so) always produce a final spin which is very close to the spin resulting from equal-mass non-spinning $\mathrm{BH}$ binaries, i.e. $a / M \sim 0.69$. Furthermore, in all three scenarios the most likely spin resulting from "major" mergers with $q \gtrsim 0.1$ is quite close to $\left|\mathrm{j}_{\text {fin }}\right| \simeq 0.69$.

A critical assessment of the available predictions on the final spin and on the final kick is outside the scope of this review; the interested reader is referred to Ref. 541.

\subsection{Astrophysical black holes: mass and spin estimates}

From the discussion in the previous section it should be clear that ringdown detectability depends crucially on the physical parameters of astrophysical BHs. 
What are the most promising scenarios leading to the formation of massive stellarmass BHs that would predominantly be seen in merger/ringdown by Earth-based interferometers? What are the event rates for mergers to be observed by LISA? Given that ringdown detectability scales strongly with mass ratio, what is the most likely mass ratio for binaries whose merger is detectable by LIGO and LISA? What is the most likely spin magnitude of BHs in binaries, and what are the odds that we can unveil connections between merger/ringdown waveforms and the central engine of GRBs? In this section we briefly survey theoretical expectations and state-of-theart measurements of the mass and spin distribution of BHs. We summarize some of the most relevant information that astronomers have collected by working as busy bees over the past thirty years, paying particular attention to the implications for the detection of gravitational waves from the merger/ringdown of $\mathrm{BH}$ binaries.

Our focus here is on testing the $\mathrm{BH}$ nature of astrophysical objects by gravitational wave observations, but there are excellent reviews on measuring $\mathrm{BH}$ masses, spins and (possibly) providing evidence of an event horizon by "traditional" electromagnetic astronomy. Narayan reviews the status of BH astrophysics, focusing on observational progress in measuring mass and spin and on (circumstantial) observational evidence for the defining property of a $\mathrm{BH}$ : the event horizon 8 . Psaltis discusses how electromagnetic observations of $\mathrm{BHs}$ and neutron stars can be used to probe strong-field gravity; in the process he describes various ways of identifying BHs and measuring their properties, including continuum spectroscopy, line spectroscopy, and attempts at imaging the vicinity of BHs to constrain their angular size [32].

Stellar-mass black hole candidates The most accurate mass measurements for stellarmass $\mathrm{BH}$ candidates are made via dynamical methods, that is, by looking at how the unseen $\mathrm{BH}$ affects the orbit of a companion star. Consider a test particle in circular orbit around the $\mathrm{BH}$. If the orbit is wide enough for Newtonian physics to apply, then the mass $M=\omega^{2} r^{3}=v^{2} r=v^{3} / \omega$, where $r$ is the orbital separation, $v$ is the orbital velocity and $\omega=2 \pi / T$ with $T$ the orbital period (simple modifications can account for orbital eccentricity). By measuring any two of $v, r$ and $\omega$, we may estimate the $\mathrm{BH}$ mass $M$. In the case of BH X-ray binaries it is relatively easy to measure $\omega$ and the maximum line-of-sight Doppler velocity $K_{c}=v \sin \iota$ of the companion star. From these quantities one can compute the "mass function"

$$
f(M) \equiv \frac{K_{c}}{\omega}=\frac{M \sin ^{3} \iota}{\left(1+M_{c} / M\right)^{2}},
$$

where $M, M_{c}$ are the masses of the $\mathrm{BH}$ candidate and of the companion, respectively. The inclination angle $\iota$ of the orbit can be estimated from the light curve of the binary, and sometimes it's even possible to estimate $M_{c}$. By combining measurements of $\omega$ and $K_{c}$ with estimates of $\iota$ and $M_{c}$, one can in principle determine the masses of both binary members. However, to identify BH candidates the essential point is to note that the mass function $f(M)$, which depends only on $\omega$ and $K_{c}$, provides a strict lower bound on $M$. Since NSs cannot be more massive than about $3 M_{\odot}$ [542, all X-ray binaries for which $f(M) \gtrsim 3 M_{\odot}$ should contain a BH. Remillard and McClintock 464 review the phenomenology of $20 \mathrm{X}$-ray binaries with dynamically confirmed $\mathrm{BHs}$, presenting a census of $\mathrm{BH}$ candidates and a critique of different methods for measuring spins. Their Table I provides a list of (lower bounds on) the mass of about $20 \mathrm{BH}$ candidates. The most massive stellar-mass $\mathrm{BH}$ candidate to date is IC 10 $\mathrm{X}-1$, with a minimum mass $M=23.1 \pm 2.1 M_{\odot}$ if the companion's mass $M_{c}=17 M_{\odot}$ 
$\left(M=32.7 \pm 2.6 M_{\odot}\right.$ if one trusts an estimate of $M_{c}=35 M_{\odot}$ for the companion) 513. This system is particularly interesting for gravitational wave detection, because it should become a close double BH binary with coalescence time of $\sim 2-3$ Gyr [543].

The relative importance of ringdown with respect to inspiral waves decreases for extreme mass ratios. For the stellar-mass binaries of interest for Earth-based interferometers, population synthesis codes suggest that $q$ should always be very close to unity 512. A rather speculative kind of source (in the absence of solid evidence for IMBHs) consists of the intermediate mass ratio inspiral (IMRI) of a compact object, such as a NS or BH, into an IMBH. The relatively low energy content in ringdown waves is compensated, in this case, by the fact that the ringdown frequency is close to the minimum of the Advanced LIGO noise power spectral density (see Appendix B of Ref. 544]). Another promising ringdown source for advanced Earthbased interferometers (albeit with highly uncertain event rates) are IMBH-IMBH inspirals. These binaries, if they are numerous enough to be detectable, present an interesting data analysis challenge: the initial inspiral phase could be detected by LISA, while the ringdown phase is in the optimal bandwidth for Advanced LIGO, that could therefore be used for "follow-up" ringdown searches [545].

Supermassive black hole candidates A good review of SMBH observations from a historical perspective can be found in Ref. 465. The first quasar was identified in 1963 [51], when the Kerr solution had just been discovered [50] and its astrophysical relevance was unclear. In the intervening years astronomers gathered strong observational evidence for the presence of SMBHs in the bulges of nearly all local, massive galaxies [546, 547, 548]. Reliable mass estimates are available for many of these systems. The most precise measurement comes from observations of stellar proper motion at the center of our own galaxy, indicating the presence of a "dark object" of mass $M \simeq(4.1 \pm 0.6) \times 10^{6} M_{\odot}$ [549, 550] and size smaller than about one astronomical unit [551. A Schwarzschild $\mathrm{BH}$ of the given mass has radius $R \simeq 0.081$ astronomical units, compatible with the observations. Any distribution of individual objects within such a small region (with the possible exceptions of dark matter particles or asteroids, which however should be kicked out by three-body interactions with stars) would be gravitationally unstable [552, 553. Theoretical alternatives to SMBHs (e.g., boson stars and gravastars) have been proposed by various authors, but the formation process of these hypothetical objects is unclear, and many of these exotic alternatives can be shown to be unstable [554, 555. Another accurate mass measurement comes from the motion of the gas disk at the centre of the nearby galaxy NGC 4258 [556, as monitored by radio interferometry of the waves emitted from water molecules via maser emission. The observations imply the presence of an object of mass $3.5 \times 10^{7} M_{\odot}$ within $\sim 4 \times 10^{15} \mathrm{~m}$. Other techniques include applications of the virial theorem to the velocity dispersion of stars near the galactic center [546] and reverberation mapping to obtain more crude estimates for distant, variable AGNs [557. The reader interested in a SMBH mass census can consult Graham's survey 558, listing 76 galaxies with direct SMBH mass measurements and (when available) their host bulge's central velocity dispersions. Graham also lists 8 stellar systems that could potentially host intermediate-mass BHs. For our purposes, it suffices to note that SMBHs have masses in the range $M \sim 10^{5}-10^{9} M_{\odot}$, approximately proportional to the mass of the host galaxies, $M \sim 10^{-3} M_{\text {galaxy }}$ 559. There is an almost-linear relation between the mass of a SMBH and the mass of the galactic bulge hosting it [546, 547, 548. The BH mass is also tightly correlated with other properties of the galactic bulge, such as the 
central stellar velocity dispersion $\sigma$, the bulge light concentration and the near-infrared bulge luminosity [560, 561, 558. These correlations clearly indicate that SMBHs are causally linked to the surrounding galactic environment. The growth of galaxies and SMBHs must be intertwined, and observations of the merger and ringdown of SMBHs with LISA holds great promise to clarify their formation history.

Supermassive black hole binary candidates The SMBHs harboring nearby galactic cores are expected to grow via a combination of mergers and accretion, and hierarchical merger models of galaxy formation predict that binary SMBHs should be common in the Universe [562, 563, 564]. The merger of SMBH binaries is one of the most luminous gravitational wave events in the universe, and it is the strongest conceivable astrophysical source of ringdown waves. Observational evidence for close SMBH binaries started emerging very recently, and it is one of the most exciting frontiers of relativistic astrophysics.

The formation of SMBHs during galaxy mergers is a challenging problem in theoretical astrophysics. The general scenario was outlined in a pioneering analysis by Begelman, Blandford and Rees 565] (see Ref. [562 for a more recent review). The evolution of a SMBH binary can be roughly divided in three phases: i) as the galaxies merge, SMBHs sink to the center via dynamical friction; ii) the binary's binding energy increases because of gravitational slingshot interactions, i.e. the ejection of stars on orbits intersecting the binary (these stars' angular momentum must be in a region of phase space called the "loss cone"); iii) if the binary separation becomes small enough, gravitational radiation carries away the remaining angular momentum. Notice that the gravitational wave coalescence time is shorter for more eccentric binaries 566, so high-eccentricity binaries are slightly more likely to coalesce within a Hubble time (see e.g. [567]). The transition from phase ii) to phase iii) is a field of active research, that has been referred to as the "final parsec problem" 562. Since the binary will quickly eject all stars through gravitational slingshot interaction, the problem is to find some mechanism (such as gas accretion, star-star encounters and triaxial distortions of galactic nuclei) to refill the loss cone. It is generally believed, based on both theoretical and observational arguments, that efficient coalescence should be the norm [567]. The main point here is that only SMBH binaries with separations $\lesssim 1$ pc can merge within a Hubble time under the sole influence of gravitational radiation.

The mass ratio distribution is an important variable from the point of view of ringdown detection. The impact of different SMBH assembly models on the mass and mass ratio distribution of detectable binaries has been discussed by various authors. The general consensus is that mass ratios $q \lesssim 1 / 10$ (and down to $q \approx 10^{-3}-10^{-4}$ ) should be common [568, 569, 570, 571, 572, 573. This may not be true for mergers between BHs in separate dark matter halos, because the smaller halo could get tidally stripped and it would not be able to sink efficiently toward the center of the main halo [574. In any case, SMBH mergers will be strong LISA ringdown sources even for modest mass ratios.

Possible observational smoking guns of SMBH binaries (such as X-shaped radio galaxies, double-double radio galaxies, helical radio jet patterns, semi-periodic signals in lightcurves, double-peaked emission-line profiles and galaxies which lack central cusps) are reviewed by Komossa [575. At the time of her review, the most spectacular example of a SMBH binary was the ultraluminous infrared galaxy NGC 6240 576, containing two active SMBHs separated by a relatively short projected distance $\sim 1 \mathrm{kpc}$. Since then, more quasars have been identified as promising hosts of SMBH 
binaries $\amalg$. Even more interestingly, in the last year three systems have been proposed to host binary SMBHs at separations smaller than one parsec [583, 584]. These observations could be extremely important for our understanding of SMBH binary mergers and their rates 585. Since they are still controversial and they would take us too far from the main topic of this review, we briefly summarize them for the interested reader in Appendix C.

Spins: theoretical expectations Estimating $\mathrm{BH}$ spins is an exciting observational frontier for the next decade of observational astronomy [586, 587, 588. Present spin estimates based on electromagnetic observations are all, to some extent, modeldependent. Few dependable and accurate measurements are available, and there are different opinions on the expected spin magnitude of both stellar-mass BHs and SMBHs. The punchline of the theoretical work summarized in this section is as follows: 1) stellar-mass BHs should typically retain their natal spin, and so they can be used to infer information about the mechanism triggering the collapse; 2) SMBH spins encode the history of the hole, and particularly the relative importance of mergers and accretion in the hierarchical formation process responsible for growing the holes. The rest of this section gives arguments in support of these conclusions.

1) Stellar-mass black holes: Theoretical arguments suggest that stellar-mass BHs in binaries retain the spin they had at birth: neither accretion nor angular momentum extraction are likely to change significantly their mass or spin. A BH must accrete an appreciable fraction of its original mass in order to significantly change its spin. For $\mathrm{BHs}$ with low-mass companions, even the accretion of the entire companion star will only change the spin by a small fraction; for BHs with high-mass companions, even Eddington-limited accretion will only grow the BH spin by a small amount before the high-mass companion explodes [589]. Therefore the spin of stellar-mass BHs should depend mainly on their formation process.

Detailed studies of spin evolution in compact binaries have been carried out by the Northwestern group, focusing mostly on NS-BH binary systems and using progressive improvements of the STARTRACK stellar evolution code (see [512] and references therein). The evolution prior to the supernova explosion involves mass-transfer phases, which are expected to align the spins of both the $\mathrm{BH}$ and the NS progenitor, but a significant natal kick of the NS at birth is required to form a coalescing NS-BH binary [590. In general, the plane of the post-supernova orbit is tilted with respect to the pre-supernova plane, and hence tilted with respect to the $\mathrm{BH}$ spin axis by some angle $\iota$, inducing precession of the binary's orbital plane. Preliminary results suggest that precession only marginally impacts the detection of gravitational waves from the inspiral waves, but it should be significant for parameter estimation [591, 512, 592. Belczynski et al. 512 consider both BH-BH and NS-BH binaries. For NS-BH binaries

+ A binary AGN with separation $4.6 \mathrm{kpc}$ has been claimed in Arp 299 [577], and a system with projected distance $\sim 10.5 \mathrm{kpc}$ has been found in the galactic pair ESO 509-IG066 578. Evans et al. [579] reveal the AGN nature of the companion of the FRII radio source 3C 321. Bianchi et al. identify a binary AGN in Mrk 463 with projected separation $\sim 3.8 \mathrm{kpc}$ [580]. Finally, using multifrequency observations with the Very Long Baseline Array (VLBA), Rodriguez et al. 581 582 . report the discovery of a SMBH binary in the radio galaxy $0402+379$ with a total estimated mass of $1.5 \times 10^{8} M_{\odot}$ and a projected orbital separation of just $7.3 \mathrm{pc}$. This is the smallest orbital separation by more than two orders of magnitude, but even for this relatively close binary the emitted gravitational waves have frequency $\sim 2 \times 10^{-13} \mathrm{~Hz}$, way too low to be observed by LISA, and a merger time $\sim 10^{18}$ yr (much longer than the age of the Universe) if gravitational radiation is the only dissipative mechanism. 
they confirm the qualitative predictions of [589]: BHs cannot be significantly spun up by accretion in the common envelope phase. For example, only $20 \%$ of initially nonspinning BHs spin up to $a / M>0.1$, and no BHs spin up to $a / M \gtrsim 0.5$. The spin-up is even smaller for BH-BH binaries, with the highest attainable spins being very close to the initial spins of the individual BHs (see Fig. 5 and Fig. 9 in 512 ). Furthermore, the kick-induced tilt angle $\iota<45^{\circ}$ for $\sim 50 \%$ of NS-BH systems. The fraction of events that can potentially produce short-hard GRBs, and which therefore is relevant for merger/ringdown searches in association with gamma-ray bursts, is significant (of order $\sim 40 \%$ ) only if the initial BH spin $a / M \gtrsim 0.6512$.

These studies suggest that Advanced-LIGO measurements of $\mathrm{BH}$ spins in the inspiral of binaries containing stellar-mass BHs should be an excellent probe of the collapse mechanism that produced the $\mathrm{BH}$ in the first place, because the $\mathrm{BH}$ essentially retains the spin it had at birth. Furthermore, in view of the NS-BH simulations discussed in Section 9.2. NS-BH binaries where the $\mathrm{BH}$ is rapidly spinning are good candidates as central engines of GRBs, and the resulting gravitational wave signal may have a significant ringdown component. This could potentially allow a ringdownbased measurement of the final $\mathrm{BH}$ spin magnitude and direction, with interesting implications for coincident electromagnetic/gravitational observations of GRB events. 2) Supermassive black holes: Since SMBHs are expected to grow by a combination of mergers and accretion, their spin will depend on three main ingredients [593. i) the spin of "seed" BHs at birth, that in some sense defines the initial conditions for the problem; ii) the spin resulting from a binary $\mathrm{BH}$ merger; iii) the maximum spin attainable by accretion. We discuss these points, in turn, below.

i) Natal spins - Little is known observationally about the formation of the first BHs in the universe. A popular formation scenario involves the collapse of primordial, massive $\left(M \sim 30-300 M_{\odot}\right)$, metal-free Population III stars at cosmological redshift $z \sim 20$ to form primordial BHs with $M \sim 10^{2} M_{\odot}$, clustering in the cores of massive dark-matter halos [594, but details of the collapse are uncertain [595, 596. Shibata and Shapiro simulated the collapse of uniformly rotating stars supported by radiation pressure and spinning at the mass-shedding limit, finding numerically [597] that the final BH spin (independently of the progenitor mass) is $a / M \approx 0.75$, and supporting this result by analytical arguments 598. Alternative scenarios suggests that $\mathrm{BH}$ seeds would form at $z \gtrsim 12$ from low-angular momentum material in protogalactic discs [599, 600]; these seeds would have larger mass $M \sim 10^{5} M_{\odot}$, but their angular momenta depend on the dynamics of the collapsing material. Whether this "initial value problem" is relevant for the overall spin distribution of SMBHs is a matter of debate. Merger tree simulations where the accretion disk orientation is chosen randomly show that the spin distribution does indeed retain memory of the initial conditions [569, 482. However, a recent model where the BH spin directions are "linked with the galaxies" suggests that the spin distribution could be largely independent of the initial conditions 601. ii) Spin from mergers - A pioneering attempt to study massive BH spin evolution by repeated mergers, predating the 2005 numerical relativity breakthrough, is due to Hughes and Blandford 602. Extrapolating results from $\mathrm{BH}$ perturbation theory they found that "minor mergers" $(q \lesssim 1 / 10)$ of a large $\mathrm{BH}$ with an isotropic distribution of small objects tend to spin down the hole. An implementation of the results shown in the right panel of Fig. 15] within merger tree scenarios confirms these results. Furthermore, it shows that SMBH spins cluster around $a / M \sim 0.7$ if alignment and accretion are inefficient, so that the BH spin growth is dominated by mergers 482 . However, Volonteri et al. [569, 482, argued that on average accretion should dominate 
over mergers in determining the spin evolution in hierarchical SMBH formation scenarios.

iii) Spin from accretion - The details of SMBH growth by accretion are very uncertain. It is usually believed that prolonged accretion should lead to large spins 569. However, King et al. 603, 604, 605, 606, suggested that gas accretion may occur through a series of chaotically oriented episodes, leading to moderate spins $a / M \sim 0.1-0.3$. Since numerical relativity suggests that comparable-mass mergers should not be efficient at spinning up BHs, and such comparable-mass mergers are expected to be common [540], a few measurements of spins $a / M \gtrsim 0.9$ (such as the value of $a / M=0.989_{-0.002}^{+0.009}$ claimed by Brenneman and Reynolds 607] for the Seyfert 1.2 galaxy MCG-06-30-15) would imply that chaotic accretion is not the norm.
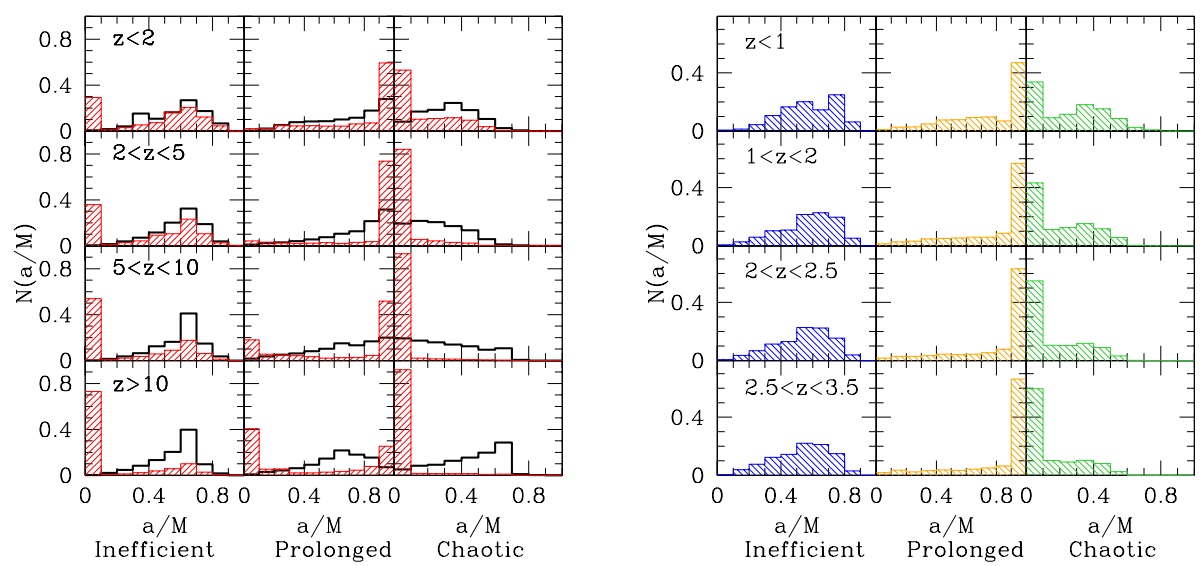

Figure 16. Left panel: spin distributions for merging BHs. Red dashed histograms show the spin distribution of inspiralling SMBHs in binaries, while black histograms show the spin distribution of SMBHs post-merger. Right panel: spin distribution of the whole $\mathrm{MBH}$ population in the mass range $10^{5} M_{\odot} \leq M \leq$ $3 \times 10^{7} M_{\odot}$. For simplicity, we consider on ly the isotropic merger scenario. Left to right in each panel, accretion is assumed to be inefficient, prolonged or chaotic, respectively (see Ref. 482 and the text for details).

Given the present uncertainty on the physical agents responsible for SMBH growth, it would be extremely valuable to find observational signatures of different formation scenarios. Figure [16 (adapted from Ref. 482]) shows that electromagnetic spin measurements and gravitational wave measurements from the inspiral and ringdown may provide an excellent way of discriminating between different mechanisms of $\mathrm{BH}$ growth. The spins are assumed to be isotropically distributed (but efficient alignment, as suggested in Ref. [540, would only marginally alter the picture: see Ref. 482 ) and the seed BHs are assumed to be non-spinning. In the left panel, the red dashed histograms show theoretical estimates of the spin distribution that could be measured by observing gravitational radiation from inspiralling $\mathrm{SMBH}$ binaries; the black histograms show the distribution of SMBH spins post-merger, as would be measured by observations of ringdown waves. Finally, the histograms in the right panel show the distribution of spins of the whole $\mathrm{BH}$ population, that can be probed by electromagnetic observations of the kind described in Appendix D. In each panel time runs upward (the histograms correspond to different redshift cuts, as 
indicated in the inset) and the three "columns", from left to right, correspond to: (1) spin growth being determined only by mergers (inefficient accretion), (2) spin growth being driven by mergers and prolonged accretion [569, (3) spin growth being driven by mergers and chaotic accretion $603,604,605$. The spin distributions are obviously very different. According to the chaotic accretion scenario, ringdown measurements would never observe $\mathrm{BH}$ spins larger than $\sim 0.7$ or so, and most spins would be very low. If prolonged accretion dominates, then most $\mathrm{BHs}$ should be rapidly spinning, and if accretion is inefficient the spin distribution should have an attractor around $j \approx 0.7$. The spin distribution clearly encodes information on the SMBH merger history.

It is important to keep in mind that the expected spins of SMBHs may well depend strongly on their masses. It has been suggested that SMBHs with $M \lesssim 2 \times 10^{6} M_{\odot}$ may grow primarily by disruption of stars (see e.g. Fig. 9 of [608]), which would then lead to low spins. These BHs would be in the optimal sensitivity window for LISA, but they are more difficult to observe electromagnetically. In contrast, the Soltan 609 argument that SMBHs grow mainly be accretion (see Appendix D) really applies only to $M \gtrsim 10^{7} M_{\odot}$, because only a small fraction of SMBH mass is in BHs with $M \lesssim 10^{7} M_{\odot}$. As a result, higher-mass SMBHs could grow mainly by accretion, but lower-mass BHs would grow mainly by stellar disruption, mergers with stellarmass compact objects and comparable-mass mergers. Therefore, the observation of spin as a function of mass could be a powerful diagnostic of SMBH evolution.

Spins: observational estimates Spin estimates based on electromagnetic observations made enormous progress in the last three years. A summary of estimates available in the literature is provided in Table 3. Notice that in some cases (most notably for 4U 1543-475 and GRO J1655-40) different methods yield sensibly different spin estimates (see e.g. 612]). The main methods used so far to estimate spins are continuum spectroscopy of accretion disks, spectroscopy of relativistically broadened Fe K $\alpha$ fluorescence lines, and energetic arguments based on the radiative efficiency of quasars. A discussion of these topics would take us too far, but it is essential to appreciate the statistical and systematic errors involved in the numbers listed in Table 3. To improve readability, we review recent literature in this field in Appendix D,

A glance at the Table shows that the estimated spin magnitudes of stellar mass $\mathrm{BHs}$ cover the whole range from zero to one. This seems to confirm the doctrine that stellar-mass BHs in X-ray binaries essentially retain the spin they had at birth [589. The situation is even more unclear for SMBHs, where (as shown in the last two lines of Table 3) uncertainties in observational data on the mean efficiency of quasars lead to very different conclusions on the average values of SMBH spins. Gravitational wave detection could be instrumental in resolving this controversy.

\subsection{Detection range for Earth-based and space-based detectors}

We usually say that a gravitational wave signal is detectable when the SNR, as defined in Eq. (152), is larger than some threshold, typically $\rho>10$. Since the gravitational wave amplitude decreases linearly with the (luminosity) distance $D_{L}$ from the source, the distance corresponding to $\rho=10$ is sometimes called the detector range. In the left panel of Fig. 17 we show LISA's SNR for equal-mass inspirals (solid lines) and the ensuing ringdown (dashed lines) as a function of the total mass of the system $M^{0}$, where the superscript " 0 " means that the mass is measured in the source frame (see Ref. 10] for details of the assumptions going into this calculation). Each line 
Table 3. A list of spin estimates available in the literature, along with the method used for the estimate (see Appendix D) and the relevant references. Tables 1 and 2 of Ref. 610 list spin estimates for 19 powerful FRII radio sources (FRIIb) and for 29 central dominant galaxies (CDGs). For MS0735.6+7421 Ref. 610] estimates a spin of $0.83 \pm 0.39$, consistent with Ref. 611.

\begin{tabular}{|c|c|c|c|}
\hline System & Estimated spin & Method & Reference \\
\hline \multicolumn{4}{|c|}{ Stellar-mass BHs } \\
\hline Cygnus X-1 & $0.05 \pm 0.01$ & Line spectroscopy & 612 \\
\hline LMC X-3 & $\approx 0.2-0.4$ & Continuum & 613 \\
\hline \multirow[t]{2}{*}{$4 \mathrm{U} 1543-475$} & $0.3 \pm 0.1$ & Line spectroscopy & 612 \\
\hline & $0.75-0.85$ & Continuum & 614 \\
\hline SAX J1711.6-3808 & $0.6_{-0.4}^{+0.2}$ & Line spectroscopy & 612 \\
\hline \multirow[t]{2}{*}{ XTE J1550-564 } & $\approx 0.1-0.8$ & Continuum & 613 \\
\hline & $0.76 \pm 0.01$ & Line spectroscopy & 612 \\
\hline SWIFT J1753.5-0127 & $0.76_{-0.15}^{+0.11}$ & Line spectroscopy & 615 \\
\hline M33 X-7 & $0.77 \pm 0.05$ & Continuum & 616 \\
\hline XTE J1908+094 & $0.75 \pm 0.09$ & Line spectroscopy & 612 \\
\hline XTE J1650-500 & $0.79 \pm 0.01$ & Line spectroscopy & 612 \\
\hline \multirow[t]{2}{*}{ GRS $1915+105$} & $0.7-0.8$ & Continuum & 617 \\
\hline & $0.98-1$ & Continuum & 618,619 \\
\hline LMC X-1 & $0.90_{-0.09}^{+0.04}$ & Continuum & 620 \\
\hline GX 339-4 & $0.94 \pm 0.02$ & Line spectroscopy & 621,612 \\
\hline \multirow[t]{4}{*}{ GRO J1655-40 } & $\geq 0.25$ & QPOs & 622 \\
\hline & $0.65-0.75$ & Continuum & 618,614 \\
\hline & $\approx 0.1-0.8$ & Continuum & 613 \\
\hline & $0.98 \pm 0.01$ & Line spectroscopy & 612 \\
\hline XTE J1655-40 & $\approx 1$ & Line spectroscopy & 623 \\
\hline XTE J1550-564 & $\approx 1$ & Line spectroscopy & 623 \\
\hline \multicolumn{4}{|c|}{ SMBHs } \\
\hline 29 CDGs & $0.1-0.8$ & Energetics & 610 \\
\hline $19 \mathrm{FRIIb}$ & $0.7-1$ & Energetics & 610 \\
\hline SWIFT J2127.4+5654 & $0.6 \pm 0.2$ & Line spectroscopy & 624 \\
\hline MCG-06-30-15 & $0.989_{-0.002}^{+0.009}$ & Line spectroscopy & 607 \\
\hline 1H0419-577 & $\approx 1$ & Line spectroscopy & 625 \\
\hline \multirow[t]{3}{*}{ MS0735.6+7421 } & $\approx 1$ & Energetics & 611 \\
\hline & Large & Average efficiency & $626,627,628,629,630$ \\
\hline & Small & Average efficiency & 631, 632, 633, 606 \\
\hline
\end{tabular}

corresponds to a different luminosity distance or, which is the same, to a different cosmological redshift $z$ : for example, a redshift $z \simeq 0.5$ corresponds to a luminosity distance of about $3 \mathrm{Gpc}$ in a standard $\Lambda \mathrm{CDM}$ cosmology. The plot illustrates a number of important points: (1) LISA can detect the last year of the inspiral of equalmass binaries with total mass $10^{3} M_{\odot}<M^{0}<10^{6} M_{\odot}$ out to cosmological distances $(z \gtrsim 10)$; (2) the ringdown phase has (under reasonable assumptions, which are confirmed by numerical simulations: see e.g. Fig. 14 in Ref. 634) a larger maximum SNR than the inspiral phase, and this maximum is achieved at larger values of the SMBH's mass. This is important, because it implies that ringdown searches are better suited for the detection of binaries with $10^{5} M_{\odot}<M^{0}<10^{7} M_{\odot}$, which is closer to 

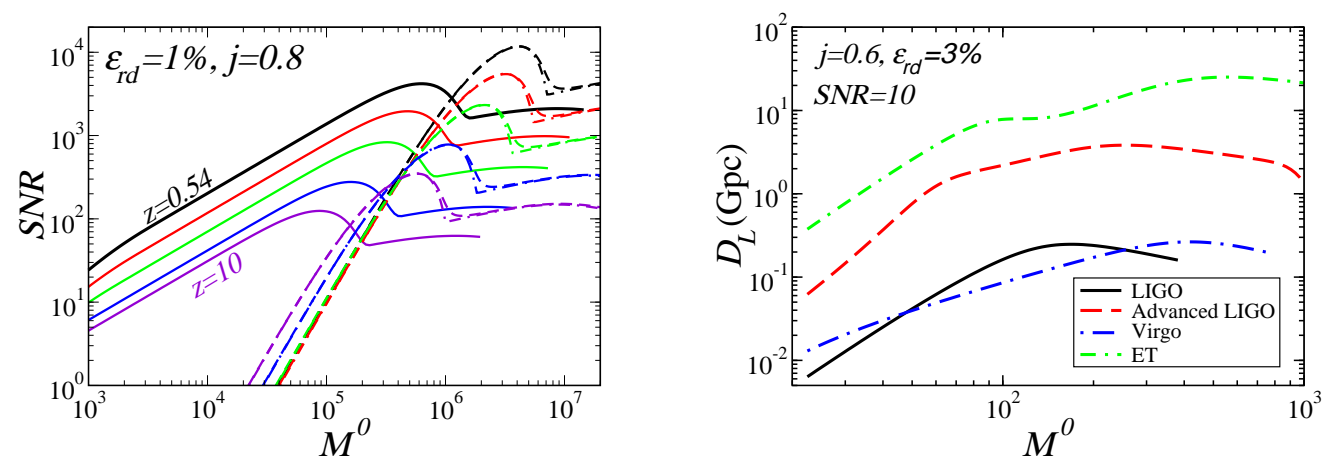

Figure 17. Left: typical SNR for equal-mass inspirals and ringdown signals detected by LISA, assuming a final spin $j=0.8$ and a conservative ringdown efficiency $\epsilon_{\mathrm{rd}}=1 \%$ (from [10]). Top to bottom, the lines correspond to sources at redshift $z=0.54,1,2,5,10$. Right: reach of Earth-based interferometers for a ringdown producing a $\mathrm{BH}$ with final spin $j=0.6$ and $\epsilon_{\mathrm{rd}}=3 \%$, as a function of the mass of the final hole (from [1]).

the typical mass range estimated for SMBHs at galactic centres (see Section 9.3).

In the right panel of Fig. 17 we show the ringdown detection range corresponding to $\rho=10$ for Earth-based detectors. The plot shows that Advanced LIGO and the Einstein Gravitational Wave Telescope (ET) have the potential to detect ringdown from IMBH-IMBH systems of mass up to $\sim 10^{3} M_{\odot}$ out to a luminosity distance of a few Gpc [545]. In fact, third-generation Earth-based interferometers could probe the first generation of "light" seed BHs of $M \sim 10^{2}-10^{3} M_{\odot}$, providing information complementary to LISA on the earliest BHs in the universe 635. A more speculative source is the intermediate-mass ratio inspiral (IMRI) of stellar-mass BHs into IMBHs. For these systems, ringdown would be suppressed relative to inspiral because of the small mass ratio. However, what is lost in terms of number of cycles is gained in terms of detector sensitivity: IMRI ringdowns would happen in the optimal frequency band of second- and third-generation detectors, and therefore they could be detectable by a network of Advanced LIGOs 544.

\subsection{Event rates}

Gravitational wave interferometers (unlike traditional electromagnetic observatories) respond to the waves' amplitude, not to their energy. Since the wave amplitude decays linearly with distance, a modest increase of (say) a factor two in sensitivity means that the detectable volume increases by a factor eight. Given that merging compact binaries are the most promising ringdown source, the relevant question for ringdown detection is then: how many merging compact binary events can we expect in a given volume? The issue of estimating event rates is one of the most pressing in gravitational wave detection. The uncertainties involved are so large, and progress in the field is so rapid, that any estimates we quote are likely to become rapidly obsolete. For this reason we dedicate little space to event rate estimates, providing a few references as a guide for the interested reader. 
Stellar-mass and intermediate-mass black hole ringdowns In the simplest models, compact binary coalescence rates should be proportional to the stellar birth rate in nearby spiral galaxies, which can be estimated from their blue luminosity. Therefore the coalescence rates are usually given in units of $\mathrm{Myr}^{-1} L_{10}^{-1}$, where $L_{10}$ is $10^{10}$ times the blue solar luminosity. To convert these numbers into detection rates, one must take into account the fact that detection ranges for ringdowns are different from those for inspirals. If the distance to an event is above $\sim 50 \mathrm{Mpc}$ the local over-density of galaxies can be ignored, and the number of galaxies containing possible sources is $N=[(4 \pi) / 3](D / \mathrm{Mpc})^{3}(2.26)^{-3} 0.0117$ Milky-way equivalent galaxies, where 2.26 is a correction factor to include averaging over all sky locations/orientations, and $0.0117 \mathrm{Mpc}^{-3}$ is the density of Milky-Way equivalent galaxies. For shorter distances one should use a sky catalog, such as Ref. 636.

For NS-NS binaries, early and conservative estimates were made by Phinney 637. At present, the most reliable NS-NS merging rate estimates are obtained by extrapolating from observed binary pulsars 638, 639. Expected rates are $\approx$ $50 \mathrm{Myr}^{-1} L_{10}^{-1}$, but they could be an order of magnitude lower or larger. For NS-BH and $\mathrm{BH}-\mathrm{BH}$ rates we have to rely mostly on population-synthesis models 640, 641. Plausible rate estimates are $\approx 2 \mathrm{Myr}^{-1} L_{10}^{-1}$ for $\mathrm{NS}-\mathrm{BH}$ binaries and $\approx 0.4 \mathrm{Myr}^{-1} L_{10}^{-1}$ for BH-BH binaries, but they could be roughly two orders of magnitude larger or lower. These rates translate into tens to thousands of inspiral events per year in Advanced LIGO. The typical end-product of these mergers are BHs of mass $\sim 10 M_{\odot}$, and the range for Advanced LIGO detection of these $\mathrm{BH}$ ringdowns is more than an order of magnitude less than the inspiral range, so ringdown rates should be $\sim 10^{2}-10^{3}$ times smaller than inspiral rates. Rates for ringdowns involving IMBHs are even more uncertain. In optimistic scenarios, Advanced LIGO could see $\sim 10$ IMBH binary mergers per year [545] and perhaps $\sim 20$ ringdowns from the merger of stellar-mass BHs into IMBHs [544] (see also 642, 643]).

These predictions rapidly change as our understanding of the underlying physics and compact binary observations improve. For example, Ref. 644 argues that potential $\mathrm{BH}-\mathrm{BH}$ binary progenitors may undergo a common envelope phase while the donor is evolving through the Hertzsprung gap. This would probably lead to a merger, thus shutting off a channel for $\mathrm{BH}-\mathrm{BH}$ production and sensibly reducing $\mathrm{BH}-$ $\mathrm{BH}$ merger rates. On the other hand, based on observations of a very massive $\mathrm{BH}$ binary, Ref. 543] estimates an initial LIGO rate of order $0.5 / \mathrm{yr}$ for relatively massive $\mathrm{BH}$ binaries (that therefore would be observed mostly in the merger/ringdown phase).

Particularly interesting for ringdown detection are compact binary mergers from dense star clusters. Ref. 645 points out that a "high-mass" selection occurs because, in nuclear star clusters at the centers of low-mass galaxies, three-body interactions usually "pair up" the two heaviest members of a triple system. This typically produces binary mergers with $M \gtrsim 20 M_{\odot}$, which means that Earth-based interferometers would usually observe the merger/ringdown phase. These findings are consistent with work by other authors 646, 647, 648].

Supermassive black hole ringdowns The LISA noise curve determines the optimal mass and redshift range where binary inspiral and ringdown events have large SNR, allowing a precise measurement of the source parameters. Reliable estimates of the number of events detectable during the mission's lifetime, and of their mass spectrum as a function of redshift, will play a key role in the planning of LISA data analysis. For this reason, over the last few years the calculation of SMBH merger event rates 
and of their mass spectrum has become an active field of research.

Table 4. SMBH binary rates (events/year) predicted by different models (adapted and updated from Ref. [567]).

\begin{tabular}{lcc}
\hline Reference & Rate & Redshift range \\
\hline Haehnelt 2003 [649] & $0.1-1$ & $0<z<5$ (gas collapse only) \\
$10-100$ & $z>5$ (hierarchical buildup) \\
\hline Enoki et al. 2004 [650] & 1 & $z>2$ \\
\hline Menou et al. 2001 [651] & 10 & $z<5$ \\
\hline Rhook and Wyithe 2005 [652, 653] & 15 & $z \sim 3-4$ \\
\hline Volonteri, Haardt and Madau [568, 570, 654] & $\sim 30$ & $z \sim 4-16$ \\
\hline Begelman, Volonteri and Rees [600, 570, 654] & $\sim 20$ & $z \sim 3-10$ \\
\hline Koushiappas and Zentner 2005 [571] & $\gtrsim 10^{3}$ & mostly $z \sim 10$, down to $z \sim 1$ \\
\hline Islam et al. 2003 [655] & $10^{4}-10^{5}$ & $z \sim 4-6$ \\
\hline
\end{tabular}

A discussion of rate estimates is out of the scope of this review (see e.g. 567. 654, 656]), but the large uncertainties in SMBH binary formation models and in the predicted event rates are quite evident from Table 4 . The numbers we list should be interpreted with caution. Each prediction depends on a large number of poorly known physical processes, and the notion of "detectability" of a merger event is defined in different ways: some authors define detectability setting a threshold on the SNR, others set a threshold on the gravitational wave effective amplitude. Furthermore, different authors use different LISA noise curves. A tentative bottom line is that we could face one of the following two scenarios. According to a class of models, we should observe $\approx 10$ events/year at redshifts (say) $2 \lesssim z \lesssim 6$. However, we cannot exclude the possibility that hundreds or thousands of SMBH binaries will produce a large (and perhaps even stochastic) background in the LISA data. Clearly, the detection strategy to use strongly depends on which of the two scenarios actually occurs in nature.

Besides being able to observe SMBH mergers throughout the universe, LISA should also be able to detect IMBH-IMBH binary mergers (that is, binaries containing a $10-100 M_{\odot} \mathrm{BH}$ orbiting a $\left.100-1000 M_{\odot} \mathrm{BH}\right)$. Rates for IMBH binary detections were first estimated by Miller 657] and then revised by Will 658. The revised estimates are very pessimistic, predicting $\sim 10^{-6}$ events/year for typical values of the parameters. A more promising scenario involves an IMBH spiralling into a SMBH 659. For these systems, Ref. 660 estimates a detection rate of a few events/year, suggesting that mergers of a $10^{3} M_{\odot}$ IMBH into a $10^{6} M_{\odot} \mathrm{SMBH}$ could be observed out to $z \sim 20$ with an SNR of 10 in a one-year integration. Ref. 661] predicts an even more optimistic rate of $\sim 10^{2}$ events/year throughout the universe. These estimates are very preliminary and even more uncertain than the corresponding estimates for SMBH binaries, but they should be taken into account in design choices concerning (for example) the optimal armlength of LISA.

\subsection{Inferring black hole mass and spin from ringdown measurements}

We have seen in Section 9.4 that the prospects for detection of ringdown radiation by LISA and advanced Earth-based detectors are quite encouraging. Interesting physics can be extracted from the observation of BH ringdowns [10, 79, 461]. Since 
astrophysical BHs in general relativity are fully characterized by their mass and angular momentum, the detection of a single mode is in principle sufficient to estimate the mass and spin parameter of the hole, by inverting the experimentally determined $\omega_{l m n}(M, a / M), Q_{l m n}(a / M)$. Indeed, one finds that accurate measurements of SMBH mass and angular momentum can be made.
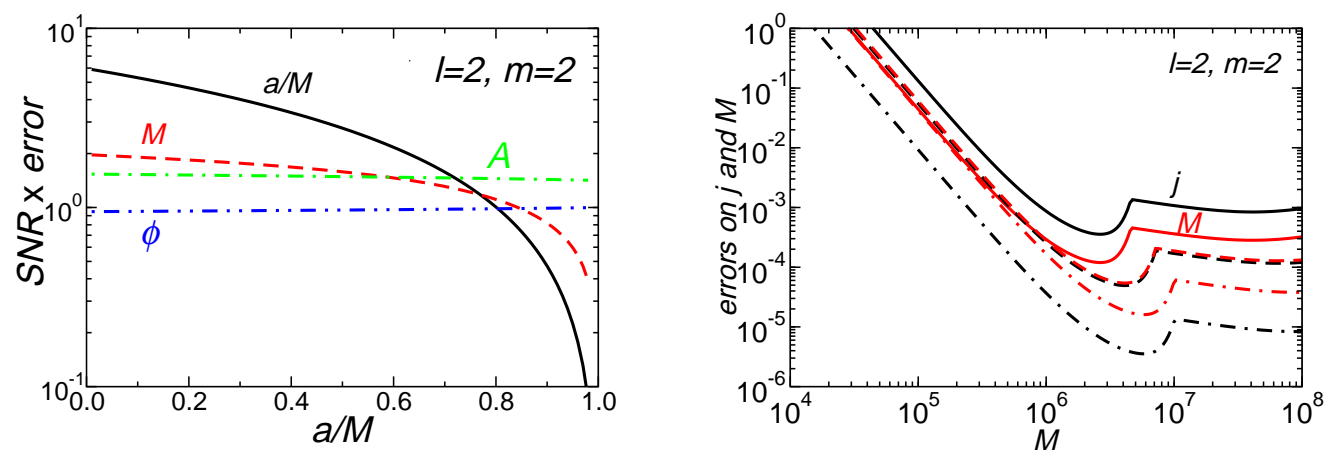

Figure 18. Left: errors (multiplied by the signal-to-noise ratio $\rho$ ) in measurements of different parameters for the fundamental $l=m=2$ mode as function of the rotation parameter $a / M$. Solid (black) lines give $\rho \sigma_{a} / M$, dashed (red) lines $\rho \sigma_{M} / M$, dot-dashed (green) lines $\rho \sigma_{A} / A$, dot-dot-dashed (blue) lines $\rho \sigma_{\phi}$, where $\sigma_{k}$ denotes the estimated rms error for variable $k, M$ denotes the mass of the $\mathrm{BH}$, and $A$ and $\phi$ denote the amplitude and phase of the wave (adapted from [10.) Right: actual errors in a LISA measurement for a source located ad $D_{L}=3 \mathrm{Gpc}$, with ringdown efficiency $\epsilon_{\mathrm{rd}}=3 \%$.

For example, the left panel of Fig. 18 shows the estimated error (multiplied by the SNR $\rho$ ) in measuring the SMBH mass $M$, angular momentum parameter $a / M$, QNM amplitude $A$, and phase $\phi$ for circularly polarized radiation from the fundamental $l=m=2$ bar mode (cf. Eq. (150) for definitions). If an energy $\sim 10^{-4} M$ is radiated into the fundamental mode of a $10^{6} M_{\odot} \mathrm{SMBH}$ with $a / M=0.8$ at $3 \mathrm{Gpc}(\rho \sim 200), M$ and $a / M$ could be measured to $1 \%$; if the energy deposition is only $10^{-6}$, they could still be measured to $10 \%$. These numbers were computed for LISA in Ref. [10, but they carry over to other detectors through a simple rescaling by $\rho$. Generalizing to multi-mode detection (and specifically to the detection of two modes with a range of relative amplitudes) one finds similar results 10. Gravitational wave detectors will be able to determine the mass and spin of BHs with excellent precision from observations of the ringdown phase.

Event loss and bias in parameter estimation using single-mode templates Current ringdown searches are performed using matched filtering and single-mode templates, consisting of a single exponentially damped sinusoid. These are the simplest possible templates, and they are expected to capture the relevant physics of the problem when one ringdown mode dominates over the others. Unfortunately, this notion of dominance must be precisely formulated. Consider for illustration the case of nonspinning binary BH mergers, and suppose for simplicity that there are only two modes in the signal, say $l=m=2$ and $l=m=3$. In particular, assume that the strain $h$ 
as seen by a detector, Eq. (151), is of the form

$$
h=\mathcal{A}_{1} e^{-\pi f_{1} t / Q_{1}} \sin \left(2 \pi f_{1} t-\phi_{1}\right)+\mathcal{A}_{2} e^{-\pi f_{2} t / Q_{2}} \sin \left(2 \pi f_{2} t-\phi_{2}\right) .(160)
$$

Estimates of the multipolar energy distribution give a relative amplitude $\mathcal{A}_{2} / \mathcal{A}_{1}=$ $h_{33} / h_{22} \sim 0.3-0.4(1-1 / q)$, where $q$ is the mass ratio and $h_{22}\left(h_{33}\right)$ are the amplitudes of the $l=m=2$ (3) modes of the radiation, respectively [44]. For $q>3$, which includes most likely merger scenarios, we get $h_{33} / h_{22} \geq 0.2-0.3$. It is now natural to ask: given a relative amplitude of this order, how many events would we miss in a search with single-mode ringdown templates?
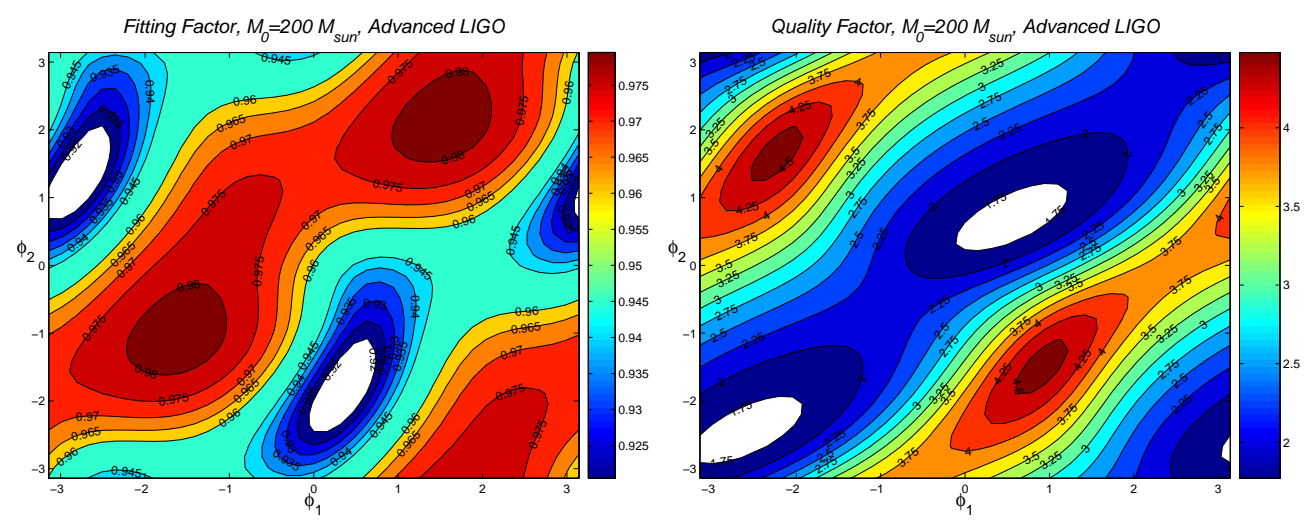

Figure 19. Left: contour plots of the fitting factor as a function of the phase angles of the signal. For this illustrative calculation we assume that the Kerr parameter of the final $\mathrm{BH}$ is $j=0.6$, and that the relative amplitude of the second mode is $\mathcal{A}=0.3$. Right: quality factor estimated by a single-mode filter. The "true" frequencies and quality factors for $a / M=0.6$ are $M \omega_{R 1}=0.4940$, $Q_{1}=2.9490, M \omega_{R 2}=0.7862, Q_{2}=4.5507$. See 11 for more details.

The answer is quantified in the left panel of Fig. 19, where we show Apostolatos' fitting factor (FF) 662, 663 resulting from searching a two-mode signal with singlemode templates from a $200 M_{\odot}, a / M=0.6 \mathrm{BH}$, with relative amplitude $A_{2} / A_{1}=0.3$, in Advanced LIGO data. Contour plots of the FF are shown as a function of the (unknown) phases in Eq. (160). The FF is essentially the ratio $\rho / \rho_{\max }$, where $\rho$ is the actual SNR achieved by matched filtering and $\rho_{\max }$ is the maximum possible SNR, attained when the template and waveform coincide. FFs larger than 0.97 , leading to a loss of less than about $10 \%$ in events, are typically considered acceptable. The FF achievable by a detection is usually assumed to correspond to the minimum of the FF in the space of the unknown parameters $\left(\phi_{1}, \phi_{2}\right)$ (this is known as the "minimax" criterion). Therefore, according to this simplified calculation, single-mode templates may well lead to unacceptable SNR degradation in a search. The situation can be even worse for larger masses and other detectors [11]; similar conclusions apply also to LISA, albeit in a completely different mass range.

Besides leading to a loss in the number of events, single-mode templates lead to a large bias in the estimation of the BH's mass and spin. The estimated frequency has relatively small bias, and it always corresponds to the dominant (least-damped) mode in the pair. Results are more interesting for the estimated quality factor, shown in the right panel of Fig. 19] as a function of the phase angles. For our chosen value 
of the Kerr parameter the quality factor of the $l=m=2$ and $l=m=3$ modes are $Q_{1}=2.9490$ and $Q_{2}=4.5507$, respectively. Comparing with the left panel, we see that relative minima in the FF (white "islands" in the left panel) occur, roughly speaking, when the quality factor "best fits" the subdominant, $l=m=3$ mode. This is a rather remarkable result: the minimax filter "best fits" the subdominant mode in the pair, leading to significant bias in the estimation of the quality factor (and hence of the spin). Unfortunately, maxima in the FF do not correspond to the filter being optimally adapted to the $l=m=2$ mode. As the filter tries to maximize the SNR, the estimated value of the quality factor becomes significantly biased, and it deviates quite sensibly from the value expected for the dominant $(l=m=2)$ mode. The bottom line is, again, that single-mode filters may be useful for detection, but a multi-mode post-processing will be necessary for accurate spin measurements.

There is, of course, a price to pay when using multi-mode templates. About $\mathcal{N} \sim 6 Q_{\max } \log f_{\max } / f_{\min } \sim 500$ single-mode templates are enough to cover the parameter space for Earth-based detector searches, if we assume an event loss of no more than $10 \%$ (i.e., a minimal match larger than 0.97663 ). For two-mode templates, rough estimates suggest that this number may increase up to $\mathcal{N} \sim b \times 10^{6}$, with $b \sim 1$ a detector-dependent constant [11. A more detailed data analysis study (e.g. using better template placing techniques, along the lines of [472, 473, 474, 13]) will be needed to reduce computational requirements.

\subsection{Tests of the no-hair theorem}

The fact that all information is radiated away in the process leading to $\mathrm{BH}$ formation, so that astrophysical BHs in Einstein's theory are characterized completely by their mass and angular momentum, is known as the "no-hair theorem". To test this theorem, it is necessary (but not sufficient) to resolve two QNMs [10, 461]. Roughly speaking, one mode is used to measure $M$ and $a$, and the other to test consistency with the Kerr solution.

Can we tell if there really are two or more modes in the signal, and can we resolve their parameters? Physical intuition suggests that if the noise is large and the amplitude of the weaker signal is very low (or if the two signals have almost identical frequencies) the two modes could be difficult to resolve. A crude lower limit on the SNR required to resolve frequencies and damping times was presented in Refs. [10, 11. The analysis uses the statistical uncertainty in the determination of each frequency and damping time, which a standard Fisher-matrix calculation estimates to be [10]

$$
\begin{aligned}
& \rho \sigma_{f_{1}}=\frac{\pi}{\sqrt{2}}\left\{\frac{f_{1}^{3}\left(3+16 Q_{1}^{4}\right)}{\mathcal{A}_{1}^{2} Q_{1}^{7}}\left[\frac{\mathcal{A}_{1}^{2} Q_{1}^{3}}{f_{1}\left(1+4 Q_{1}^{2}\right)}+\frac{\mathcal{A}_{2}^{2} Q_{2}^{3}}{f_{2}\left(1+4 Q_{2}^{2}\right)}\right]\right\}^{\frac{1}{2}}, \\
& \rho \sigma_{\tau_{1}}=\frac{2}{\pi}\left\{\frac{\left(3+4 Q_{1}^{2}\right)}{\mathcal{A}_{1}^{2} f_{1} Q_{1}}\left[\frac{\mathcal{A}_{1}^{2} Q_{1}^{3}}{f_{1}\left(1+4 Q_{1}^{2}\right)}+\frac{\mathcal{A}_{2}^{2} Q_{2}^{3}}{f_{2}\left(1+4 Q_{2}^{2}\right)}\right]\right\}^{\frac{1}{2}} .
\end{aligned}
$$

These errors refer to mode " 1 " in a pair; errors on $f_{2}$ and $\tau_{2}$ are simply obtained by exchanging indices $(1 \leftrightarrow 2)$ [10, 11. The expressions above further assume white noise for the detector, and that modes " 1 " and " 2 " correspond to different values of $l$ or $m$.

A natural criterion (á la Rayleigh) to resolve frequencies and damping times is that $\left|f_{1}-f_{2}\right|>\max \left(\sigma_{f_{1}}, \sigma_{f_{2}}\right),\left|\tau_{1}-\tau_{2}\right|>\max \left(\sigma_{\tau_{1}}, \sigma_{\tau_{2}}\right)$. In interferometry this would mean that two objects are (barely) resolvable if the maximum of the diffraction pattern of object 1 is located at the minimum of the diffraction pattern of object 2 . 
We can introduce two "critical" SNRs required to resolve frequencies and damping times, $\rho_{\text {crit }}^{f}=\max \left(\rho \sigma_{f_{1}}, \rho \sigma_{f_{2}}\right) /\left|f_{1}-f_{2}\right|, \rho_{\text {crit }}^{\tau}=\max \left(\rho \sigma_{\tau_{1}}, \rho \sigma_{\tau_{2}}\right) /\left|\tau_{1}-\tau_{2}\right|$, and recast our resolvability conditions as

$$
\begin{aligned}
& \rho>\rho_{\text {crit }}=\min \left(\rho_{\text {crit }}^{f}, \rho_{\text {crit }}^{\tau}\right), \\
& \rho>\rho_{\text {both }}=\max \left(\rho_{\text {crit }}^{f}, \rho_{\text {crit }}^{\tau}\right) .
\end{aligned}
$$

The first condition implies resolvability of either the frequency or the damping time, and the second implies resolvability of both.

Now let us consider how to resolve amplitudes, i.e. the minimum SNR needed to determine whether two or more modes are present in a given ringdown signal. Suppose again, for simplicity, that the true signal is a two-mode superposition. Then we expect the weaker signal to be hard to resolve if its amplitude is low and/or if the detector's noise is large. Appendix B of Ref. 11] quantifies this statement by deriving a critical SNR for amplitude resolvability $\rho_{\text {GLRT }}$ based on the generalized likelihood ratio test. The derivation of this critical SNR is based on the following simplifying assumptions: (i) using other criteria one has already decided for the presence of at least one damped exponential in the signal, and (ii) the parameters of the ringdown signal (frequencies and damping times), as well as the amplitude of the dominant mode, are known. In practice the latter assumption is not valid, so these estimates of the minimum SNR should be considered optimistic. Fig. 20 compares the critical SNR $\rho_{\text {GLRT }}$ (Eq. (B12)

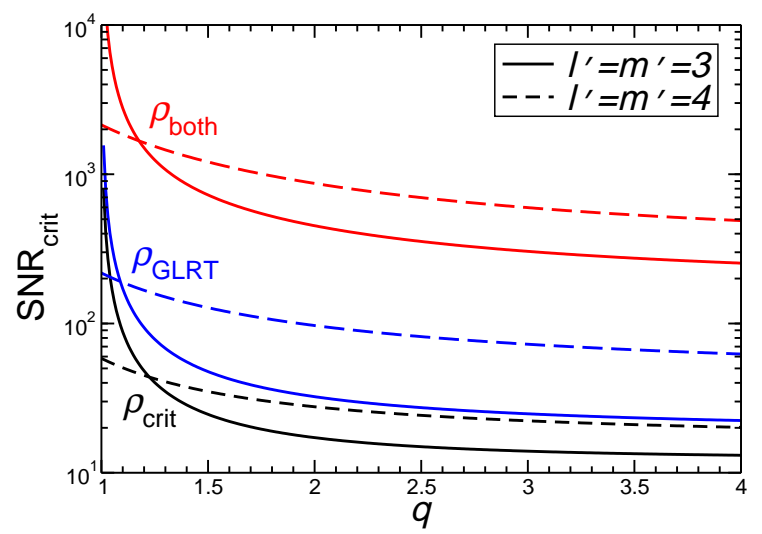

Figure 20. Minimum SNR required to resolve two QNMs from a BH resulting from the inspiralling of a BH binary with mass ratio $q$. The dominant mode (mode "1") is assumed to be the fundamental QNM with $l=m=2$; mode "2" is either the fundamental $l=m=3$ QNM (solid lines) or the fundamental $l=m=4$ QNM (dashed lines). The relative mode amplitude $\mathcal{A}(q)$ is estimated from numerical simulations 44. If $\rho>\rho_{\mathrm{GLRT}}$ we can tell the presence of a second mode in the waveform, if $\rho>\rho_{\text {crit }}$ we can resolve either the frequency or the damping time, and if $\rho>\rho_{\text {both }}$ we can resolve both.

of Ref. [1] ) and the two different criteria for frequency resolvability, Eqs. (163) and (164). The plot shows that $\rho_{\text {crit }}<\rho_{\text {GLRT }}<\rho_{\text {both }}$ for all values of $q$. Therefore, given a detection, the most important criterion to determine whether we can carry out no-hair tests is the GLRT criterion. If $\rho>\rho_{\text {GLRT }}$ we can decide for the presence of a second 
mode in the signal. Whenever the second mode is present, we also have $\rho>\rho_{\text {crit }}$ : that is, we can resolve at least the frequencies (if not also the damping times) of the two modes. A SNR $\rho \sim 30-40$ is typically enough to perform the GLRT test on the $l=m=3$ mode, as long as $q \gtrsim 1.5$. From Figs. 17] 20] we conclude that not only LISA, but also advanced Earth-based detectors (Advanced LIGO and ET) have the potential to identify Kerr BHs as the vacuum solutions of Einstein's general relativity.

In conclusion, ringdown radiation can be used to distinguish BHs from exotic alternatives, such as boson stars 463 or gravastars 664. Ringdown tests of the Kerr nature of astrophysical BHs are independent from (and complementary to) proposed tests using a multipolar mapping of the Kerr spacetime, as encoded in EMRI signals according to "Ryan's theorem" and its generalizations [665, 666, 667].

\subsection{Matching inspiral and ringdown: problems and applications}

Models of gravitational waveforms from inspiralling compact binaries usually rely on the post-Newtonian approximation to general relativity 668. Ideally, for matchedfiltering detection we would like to have detection template banks with "complete" waveforms encompassing the inspiral, merger and ringdown. Phenomenological template families based on physically motivated fits of numerical merger waveforms or on effective-one-body models are now available [669, 634, 670, 671]. However, it would be desirable to have a full understanding of the merger process, connecting the post-Newtonian approximation during the inspiral to a description of the ringdown as a superposition of QNMs.

QNM fits of numerical relativity waveforms are routinely used to check that the total angular momentum of the system is conserved during $\mathrm{BH}$ merger simulations. This is usually achieved by computing three independent quantities: the angular momentum radiated at infinity, the angular momentum obtained from isolated horizon calculations, and the angular momentum obtained by inverting the frequencies and damping times resulting from a QNM fit of the ringdown of the final Kerr BH (see e.g. 477, 44, 672]). Buonanno, Cook and Pretorius noticed that, as more and more overtones are included, a QNM expansion gets in better agreement with numerical waveforms for equal-mass binaries [477.

So far, efforts to "stitch" the post-Newtonian approximation (or one of its effective-one-body variants) to the ringdown have used rather crude models for ringdown excitation. The original effective-one-body approach simply attached the plunge waveform to the fundamental $l=m=2 \mathrm{QNM}$ of a Kerr BH 673. In their study of recoils, Damour and Gopakumar attached the plunge waveform to the fundamental Schwarzschild QNMs with $l=2$ and $l=3$ 674. A comprehensive analysis of Pretorius' numerical waveforms for equal-mass BH binaries [477] clearly illustrated the importance of higher-order overtones. Damour and Nagar obtained a good match to numerical relativity waveforms by requiring continuity of the plunge waveform with a (Schwarzschild) ringdown waveform including five overtones on a grid of points, or "comb" [176]. A "hybrid comb" procedure, imposing the continuity of the waveform and its derivatives, was later introduced in Ref. 174. These stitching methods have great phenomenological interest, and they can do remarkably well at reproducing numerical waveforms. For example, the authors of Ref. [174 extend an effective-one-body waveform through merger by stitching the inspiral waveform to QNMs, finding striking agreement (at the $0.1 \%$ level) between the numerical QNM frequency and the perturbative prediction for the same frequency computed from the 
final mass and spin of the numerical simulation. A slightly different approach was adopted in Ref. 670, where the frequency-domain waveform was truncated abruptly at some cutoff frequency close to the QNM frequency. The truncated waveforms display a Gibbs phenomenon in the time domain, and they are (surprisingly) quite effective at detecting the full signal. The reason is that a cutoff in the frequency domain around the fundamental QNM frequency corresponds, in the time domain, to a damped sinusoid with frequency close to the cutoff frequency.

These phenomenological "stitching recipes" may well be enough for the purpose of gravitational wave detection, but it is desirable to have a complete understanding of QNM excitation in a compact object merger within perturbation theory, based on the concept of excitation coefficients. Ref. [167] carried out a first step in this direction by computing the excitation factors for Kerr BHs (see Section 3.2), but more work is required to compute the excitation coefficients for generic initial data, and to understand the validity of the linear approximation in a $\mathrm{BH}$ merger. A correct matching of effective-one-body waveforms with the ringdown signal may have astrophysical implications because QNMs play an important role in the recoil of merging BH binaries, through a process sometimes called "ringdown braking" (see 675, 674, 676 and references therein). A complete analytical description of the merger of spinning, precessing binaries would also prove useful for statistical studies of astrophysical relevance 677.

Even if phenomenological waveforms may be good enough at detecting gravitational wave signals, a complete description of the waveform (including both inspiral and ringdown) has been shown to improve parameter estimation by effectively decorrelating the source parameters (see 534 for a study predating the numerical relativity breakthrough, and 678, 679, 669, 634, 535. for recent efforts in this direction). Large-scale efforts (dubbed the NINJA and Samurai projects, respectively) are now attempting to use complete numerical waveforms in LIGO data analysis, and to cross-validate numerical waveforms produced by different codes [525, 680].

An important point to keep in mind is that inspiral and merger/ringdown really probe different $\mathrm{BH}$ populations, so they provide complementary information. This should be quite clear by inspecting the red and black histograms in Fig. 16. which show that the spin distribution of SMBHs in binaries is usually different from the spin distribution of the remnant $\mathrm{BHs}$ formed as a result of the merger. This complementarity can be used for interesting physical applications. For example, if we can determine accurately enough the masses and spins of BHs before and after merger for the same system, we could use this information to test Hawking's area theorem in astrophysical settings 681].

\section{Other recent developments}

\subsection{Black hole area quantization: in search of a $\log$}

One of the main driving forces behind the development of new tools to study QNMs is the possibility that classical $\mathrm{BH}$ oscillations could yield insights into their quantum behavior. First suggested by York 68 and Hod [17, this idea was further explored by Dreyer [97] in the context of Loop Quantum Gravity, and subsequently revisited by many authors (see e.g. 682, 276, 683, 684, 685, 686, 687, 688, 689, 690, 691]).

The idea can be traced back to arguments by Bekenstein and collaborators 692, 693]. A semi-classical reasoning suggests that the $\mathrm{BH}$ area spectrum is quantized 
according to

$$
A_{N}=\gamma l_{P}^{2}, \quad N=1,2, \ldots,
$$

where $l_{P}$ is the Planck length (but see 694 for criticism). Statistical physics arguments impose a constraint on $\gamma$ 693, 695]:

$$
\gamma=4 \log k,
$$

where $k$ is an integer, which in general is left undetermined (but see 696]). Inspired by Bekenstein's ideas, Hod [17] proposed to determine $k$ via a version of Bohr's correspondence principle in which the highly-damped QNM frequencies play a fundamental role. At the time, the only available exploration of highly-damped QNMs was the numerical study by Nollert [90, which indicated that gravitational highly-damped QNMs in the Schwarzschild geometry asymptote to

$$
M \omega=0.0437123-i(2 n+1) / 8 .
$$

While looking for classical oscillation frequencies proportional to the logarithm of an integer, Hod realized that $0.0437123 \sim \ln 3 /(8 \pi)$, and went on to suggest that the emission of a quantum with frequency $\omega_{R}=\ln 3 /(8 \pi)$ corresponds to the smallest energy a $\mathrm{BH}$ can emit. The corresponding change in surface area would then be

$$
\Delta A=32 \pi M d M=32 \pi M \hbar \omega=4 \hbar \ln 3 .
$$

and by comparing with Eq. (165) we get $k=3$, thus fixing the area spectrum to

$$
A_{\mathrm{n}}=4 l_{P}^{2} \ln 3 n ; n=1,2, \ldots
$$

A few years later Dreyer 97] used similar arguments to fix the Barbero-Immirzi parameter of Loop Quantum Gravity. Shortly afterwards, Motl [91] and Motl and Neitzke [101 showed analytically that the highly damped QNM frequencies of a Schwarzschild $\mathrm{BH}$ are indeed given by Eq. (86), containing the desired logarithm of an integer.

Motl and Neitzke's work lent some support to Hod's ideas. It was followed by a flurry of activity to explore the highly-damped QNMs of several BH spacetimes, whose outcome has been described and summarized in previous sections. Unfortunately the conjectured relation between QNMs and area quantization stumbled before the charged and rotating four-dimensional geometries, for which the highly-damped regime is not as simple as suggested by Hod's original argument (see Sections 5.3 5.2). Recently Maggiore 688 observed that Hod's prescription was based on experience with "normal" quantum systems, for which the relevant frequency is $\omega_{R}$. For highlydamped systems, Maggiore noted that one should rather consider the imaginary part of the QNMs $\omega_{I}$, solving a number of puzzles and obtaining a different expression for the quantum of area. A similar prescription has been extended also to Kerr BHs 689, 690 and other geometries 691]. It is not clear whether Maggiore's suggestion can be extended consistently to all geometries. Whether a relation between QNMs and the quantum behavior of BHs exists or not, Hod's suggestion was at the very least an important thrust to complete our understanding of classical BH oscillation spectra.

\subsection{Thermodynamics and phase transitions in black hole systems}

In the last few years, remarkable relations between classical and thermodynamical properties of black objects have been uncovered. For instance, a correspondence between classical and thermodynamical instabilities of a large class of black branes 
conjectured by Gubser and Mitra [697, 698, was proved by Reall 699] (see [700] for a review). Manifestations of this duality are expected to appear in the QNM spectra. Indeed, some indications that phase transitions correspond to changes in the QNM spectrum were provided in specific cases by various authors [701, 702, 703, 704, 705, 706. However, at present there seems to be no obvious correspondence between thermodynamical phase transitions of the kind suggested by Davies [707, 708] and QNM spectra (see [709] and references therein).

Another connection between QNMs and $\mathrm{BH}$ thermodynamics may follow from Hod's proposed "universal relaxation bound" 710, 711, 712. Hod's proposal asserts that the relaxation time $\tau$ of any thermodynamic system is bounded by $\tau \geq \hbar /(\pi T)$, where $T$ is the temperature of the system. For BHs this implies the existence of (at least one) QNM frequency with imaginary part $\omega_{I} \leq \pi T$, where $T$ is now the Hawking temperature of the $\mathrm{BH}$. The bound seems to be valid for various kinds of BHs, and it may be saturated by extremal BHs. The significance of Hod's bound is not completely clear to us. The bound is trivially satisfied by any physical system exhibiting hydrodynamic behavior, since such a system always possesses sufficiently long-lived modes (and, correspondingly, QNMs with imaginary part sufficiently close or even infinitely close to zero: these hydrodynamic frequencies are discussed in Section 8.3). Finite-volume systems might be more interesting, but then the concept of the "relaxation time" used in the bound needs a proper definition. In any case, this is an interesting idea which might require better understanding. The relation between Hod's bound and the viscosity-entropy bound (see Section 8.4) was discussed in Ref. [713].

\subsection{Non-linear quasinormal modes}

QNMs are usually defined and studied by considering only first-order perturbations. Being an intrinsically non-linear theory, general relativity is expected to display nonlinear effects, which might conceivably be captured by going to higher orders in perturbation theory. The second-order formalism laid down several years ago by various authors [85, 714, 715] has recently been used to compute corrections to the QNM frequencies, their detectability and their influence on the late-time behavior of the system [716, 717, 718. The encouraging outcome of these studies is that nonlinear effects may well be observable by future gravitational wave interferometers. Favata [719] explores the interesting possibility of detecting another important nonlinear effect of general relativity (the so-called gravitational wave memory) through gravitational wave observations of merging binaries.

Higher-order perturbations of $\mathrm{BH}$ spacetimes have been explored systematically by Brizuela et al. [720, 721, using the Gerlach-Sengupta formalism and the computer algebra methods described in [722] (see also Kol [723]). A complete gauge-invariant formalism for second-order perturbations of Schwarzschild BHs was recently reported 724.

\subsection{Quasinormal modes and analogue black holes}

Strong-field effects of general relativity are very small in Earth-bound experiments. For this reason BH physics is most easily studied via observations of astrophysical phenomena such as accretion, X-ray spectra, and hopefully gravitational wave emission. The possibility to devise gravitational experiments probing strong-field general relativity in the lab, as appealing as it sounds, may seem out of reach. However, 
Unruh, Visser and others [725, 31] ingeniously showed that some defining properties of BHs can be reproduced and studied by "analogue BHs". These systems display at least a subset of the properties traditionally associated with BHs and event horizons. Unruh's analogue BHs do not carry information about the dynamics of Einstein's equations, but share many kinematical features with true general-relativistic BHs.

The basic idea behind these analogue $\mathrm{BHs}$ is quite simple. Let us focus on a particular analogue $\mathrm{BH}$, the acoustic or "dumb" hole [725, 726]. Consider a fluid moving with a space-dependent velocity $v_{0}^{i}\left(x^{i}\right)$, for example water flowing through a variable-section tube. Suppose the fluid velocity increases downstream, and that there is a point where the fluid velocity exceeds the local sound velocity $c\left(x^{i}\right) \equiv \sqrt{\partial p / \partial \rho}$, in a certain frame. At this point, in that frame, we get the equivalent of an apparent horizon for sound waves. In fact, no (sonic) information generated downstream of this point can ever be communicated upstream (for the velocity of any perturbation is always directed downstream, by simple velocity addition). This is the acoustic analogue of a $\mathrm{BH}$, sometimes referred to as a dumb hole. These are not true BHs, because the acoustic metric satisfies the equations of fluid dynamics and not Einstein's equations. However, sound waves propagate according to the usual curved space KleinGordon equation with the effective metric [725, 726, 31,

$$
g^{u \nu} \equiv \frac{1}{\rho_{0} c}\left[\begin{array}{ccc}
-1 & \vdots & -v_{0}^{j} \\
\ldots \ldots & . & \ldots \ldots . \\
-v_{0}^{i} & \vdots & \left(c^{2} \delta_{i j}-v_{0}^{i} v_{0}^{j}\right)
\end{array}\right] .
$$

Analogue BHs should Hawking-radiate, though an experimental verification of Hawking radiation in the lab is not an easy feat 727, 31. Furthermore, sound wave propagation in these metrics should reproduce many features of wave propagation in curved spacetimes. Most importantly for this review, acoustic BHs have a (this time literally!) "characteristic sound" [5] encoded in their QNM spectrum. QNMs of acoustic BHs, which may be important in experimental realizations of the idea, were computed in Refs. 728, 240, 729, 730, for a simple $(2+1)$-dimensional acoustic hole, the "draining bathtub" [726], for which $\vec{v}_{0}=(-A \vec{r}+B \vec{\phi}) / r$. The modes of $(3+1)$-dimensional acoustic holes with a "sink" at the origin were computed in Refs. [728, 731, 732]. Scattering from these holes is discussed in Ref. 733].

The experimental confirmation of these predictions is an interesting topic for future research. In practice, one may need a device to accelerate the fluid up to supersonic velocities, such as a Laval nozzle [734, 735] (see also Unruh's discussion in [727]). Laval nozzles were first used in steam turbines, but they find applications in other contexts, including rocket engines and nozzles in supersonic wind tunnels. They consist of a converging pipe where the fluid is accelerated, followed by a throat (the narrowest part of the tube) where the flow undergoes a sonic transition, and finally a diverging pipe where the fluid continues to accelerate. QNMs of flows in de Laval nozzles were discussed and computed in Refs. 736, 737.

Following on Unruh's "dumb hole" proposal, many different kinds of analogue BHs have been devised, based on condensed matter physics, slow light etcetera. We refer the reader to Refs. [727, 31] for thorough reviews on the subject. QNMs of condensed-matter analogue BHs were recently computed in Refs. [738, 739]. Finally, we should mention that accretion of material onto astrophysical $\mathrm{BHs}$ can give rise to supersonic walls, i.e., acoustic horizons outside the event horizon. This process is particularly interesting: astrophysical BHs may provide a nature-given setting for 
producing analogue BHs [740, 741].

\section{Outlook}

The investigations of the last decade show that quasinormal spectra encode a wealth of information on the classical and quantum properties of BHs and black branes. From a holographic, high-energy perspective, BH QNMs yield important information on the quasiparticle spectra and transport coefficients of the dual theory, and have an intriguing hydrodynamic description. From an astrophysical viewpoint, gravitational wave measurements of QNMs may allow us to accurately measure BH masses and spins with unprecedented accuracy and to test the no-hair theorem of general relativity. The suggestion that $\mathrm{BH}$ oscillation frequencies may be related to their quantum properties is controversial, but at the very least it has stimulated tremendous technical progress in the calculation of previously unexplored regimes of the QNM spectrum. In this review we tried to summarize these recent developments. There is no doubt that further interesting connections between QNM research, fundamental physics and astrophysics will be unveiled in the future. In closing, we think it might be useful to list some of the outstanding, unresolved issues.

General mathematical problems, asymptotic expansions and related issues - It would probably be fair to say that the level of mathematical rigor in QNM studies deserves an improvement. Whether or not boundary value problems for dissipative systems can be treated in the framework of a sufficiently general mathematical theory appears to be an open question. Less ambitiously, a unified treatment of resonances (QNMs included) at the level of mathematical physics would be desirable. Reliable regular and asymptotic expansions based on systematic procedures rather than ad hoc recipes have not yet been developed in many cases. Improving numerical algorithms, in particular for QNMs with large imaginary parts, also remains an avenue of research.

Black hole perturbation theory in asymptotically-flat spacetimes - Despite a fifty-year long history of investigation, BH perturbation theory in asymptotically flat spacetimes has a number of unresolved issues. The problem of metric reconstruction from the Teukolsky formalism is still open. QNM excitation for generic perturbations of Kerr BHs have not been fully explored yet. The eikonal limit of Kerr QNMs is still poorly understood, as is the relation of QNMs with generic $(l, m)$ to unstable circular geodesics in the Kerr metric. Finally, an outstanding problem concerns the decoupling of linear perturbations of the Kerr-Newman metric.

Gauge-gravity duality - At the moment, computing QNMs in the context of the gaugegravity duality is a thriving industry, naturally expanding to include models with higher-derivative gravity, holographic models with spontaneous symmetry breaking, or backgrounds dual to non-relativistic theories. As discussed in Section 8 , one distinguishes hydrodynamic-type modes from the rest of the QNM spectrum. On a technical level, these modes are characterized by small parameters and thus can often be determined analytically. A full analysis of hydrodynamic QNMs for generic backgrounds is currently lacking. In particular, it would be helpful to obtain an expression for the parameters of the sound mode similar to Eq. (148) for the diffusion constant. The spectra of fermionic fluctuations (e.g. of the Rarita-Schwinger field, see [417) in black brane backgrounds have not yet been fully computed. It would be very interesting to explore further the connection between holography and the black hole membrane paradigm [117, 742, 114, 115].

Gravitational wave astronomy - An important problem in gravitational wave physics 
concerns the matching of inspiral waveforms (as computed by post-Newtonian theory) to the ringdown phase. This is necessary to produce complete phenomenological templates to be used in gravitational wave detection. Most attempts at solving this problem have adopted a purely phenomenological approach, but a study of the excitation coefficients induced by generic initial data (possibly considering non-linear corrections) could provide a more consistent and systematic solution to the matching problem. A study of the QNM contribution to the Green function has recently been shown to hold promise as a computational approach to solve the self-force problem 172, which is of fundamental importance to model extreme- and intermediate-mass ratio inspirals for LISA data analysis. Other open issues concern ringdown data analysis. Simple calculations show that single-mode templates only produce moderate losses in SNR when detecting multi-mode signals. However, the loss in terms of parameter estimation accuracy is much more significant. The problem of optimal template placing for detection by multi-mode templates needs to be addressed. The potential of higher multipoles in estimating the source parameters without angleaveraging should also be explored: the different angular dependence of subdominant multipoles may provide information on the spin direction of the final BH. If BH spins are linked to jets, this information could be used for coincident searches of gamma-ray bursts or other electromagnetic counterparts to compact binary mergers 522, 523.

In the last few years, unexpected connections between QNMs and seemingly unrelated phenomena (such as analogue BHs or thermodynamical BH phase transitions) have been uncovered or proposed. More intriguing connections will surely emerge in the coming decades.

\section{Acknowledgments}

We thank Alessandra Buonanno, Manuela Campanelli, Marc Casals, Ruth Daly, Sam Dolan, Guido Festuccia, Martin Gaskell, Leonardo Gualtieri, Shahar Hod, Roman Konoplya, Cole Miller, Christian Ott, Luciano Rezzolla, Andrzej Rostworowski, Ana Sousa, Uli Sperhake and Vilson Zanchin for very useful discussions and for sharing with us some unpublished material. We also thank Ilya Mandel for clarifications on rate calculations; Luca Baiotti, Bruno Giacomazzo and Luciano Rezzolla for providing numerical data from Ref. 46; Marta Volonteri for preparing Figure 16. Lubos Motl and Andrew Neitzke for permission to reproduce Figure 4 from Ref. [101; Shijun Yoshida for providing numerical data from Ref. [234, and Alex Miranda for numerous suggestions. V.C.'s work was partially funded by Fundação para a Ciência e Tecnologia (FCT) - Portugal through projects PTDC/FIS/64175/2006, by a Fulbright Scholarship, and by the National Science Foundation through LIGO Research Support grant NSF PHY-0757937.

\section{Appendix A.}

\section{Isospectrality, algebraically special modes and naked singularities}

A number of striking relations among gravitational perturbations of the Kerr geometry were revealed by Chandrasekhar and colleagues [22, 141, 62, 142, 743. Here we review these relations specializing most of the discussion to the Schwarzschild geometry. The reader is referred to the original works for more details and for extensions to the Kerr spacetime [22, 141, 62, 142]. 
By direct substitution, it can be checked that the gravitational potentials $V_{s=2}^{ \pm}$ in Eqs. (14) and (15) can be rewritten in the form

$$
V_{s=2}^{ \pm}=W^{2} \mp \frac{d W}{d r_{*}}+\beta, \quad \beta=-\frac{\lambda^{2}(\lambda+1)^{2}}{9 M^{2}},
$$

where

$$
W=\frac{3 M\left(6 M r^{2}+2 \lambda L^{2}(2 M-r)\right)}{\lambda r^{2}(6 M+2 \lambda r) L^{2}}-\frac{\lambda(\lambda+1)}{3 M}-\frac{3 M}{\lambda L^{2}} .
$$

(these equations correct some typos in Ref. [130]). Eq. (A.1) emerged from Chandrasekhar's investigations [62, 141, 142] of the nature of the gravitational potentials. Potentials of this form are called superpartner potentials [744], and they imply the following relation between the corresponding wavefunctions $\Psi^{-}$and $\Psi^{+}$ [22, 130]:

$$
\Psi^{ \pm}=\frac{1}{\beta-\omega^{2}}\left(\mp W \Psi^{\mp}+\frac{d \Psi^{\mp}}{d r_{*}}\right)
$$

Eq. (A.1) seems to be unique to four-dimensional spacetimes, and does not generalize to higher dimensions [102, 103]. The potential for electromagnetic-type and gravitational-type perturbations of extremal $\mathrm{RN}$ BHs can also be expressed in the form A.1 259, 260. This justifies the fact that electromagnetic perturbations with angular index $l$ are isospectral to gravitational with index $l-1$, as discussed in Section 5.2. The isospectrality is a manifestation of supersymmetry between electromagnetic and gravitational perturbations for extremal charged BHs [259, 260, 261].

Isospectrality and asymptotic flatness Suppose that $\omega$ is a QNM frequency for $\Psi^{+}$, i.e. that $\Psi^{+} \rightarrow A^{+} e^{-i \omega r_{*}}$ when $r \rightarrow r_{+}$and $\Psi^{+} \rightarrow a_{\text {out }}^{+} e^{i \omega r_{*}}$ when $r \rightarrow \infty$. Here $a_{\text {out }}^{+}=1$ for the Schwarzschild metric and, if we impose Dirichlet boundary conditions, $a_{\text {out }}^{+}=0$ for the SAdS metric. Substituting into Eq. (A.3) we see that

$$
\begin{aligned}
\Psi^{-} & \rightarrow \frac{A^{+}}{\beta-\omega^{2}}\left(W\left(r_{+}\right)-i \omega\right) e^{-i \omega r_{*}}, \quad r \rightarrow r_{+}, \\
& \rightarrow \frac{1}{\beta-\omega^{2}}\left(-a_{\text {out }}^{+} W(\infty) e^{i \omega r_{*}}+\frac{d \Psi^{-}}{d r_{*}}\right), \quad r \rightarrow \infty .
\end{aligned}
$$

The key point is that $d \Psi^{-} / d r_{*} \sim e^{i \omega r_{*}}$ at infinity for the Schwarzschild geometry. Therefore, if $\omega$ is a QNM frequency for $\Psi^{+}$it is also a QNM frequency for $\Psi^{-}$, and the two types of gravitational perturbations are isospectral [62, 141]. In general $d \Psi^{-} / d r_{*} \neq 0$ for SAdS, so the isospectrality is broken. The above relations are valid also for dS backgrounds: since the outer boundary conditions are imposed at the cosmological horizon, it is easy to see that gravitational perturbations of both parities are again isospectral. A specialized analysis is needed at the points where $\beta-\omega^{2}=0$ : this condition defines the so-called algebraically special modes, discussed below.

Algebraically special modes A class of special modes can be found analytically using Eq. (A.1). In the Petrov classification, this condition corresponds to a change in the algebraic character of the spacetime. For this reason, Chandrasekhar called these modes "algebraically special" (AS) [142, 22]. Defining $\pm W=\frac{d}{d r_{*}} \log \chi_{ \pm}$, the wave equation can be written as

$$
\frac{1}{\Psi_{ \pm}} \frac{d^{2} \Psi_{ \pm}}{d r_{*}^{2}}+\left(\omega^{2}-\beta\right)=\frac{1}{\chi_{ \pm}} \frac{d^{2} \chi_{ \pm}}{d r_{*}^{2}},
$$


where we have used the identity $\frac{1}{\chi_{ \pm}} \frac{d^{2} \chi_{ \pm}}{d r_{*}^{2}}=W^{2} \pm \frac{d W}{d r_{*}}$. AS modes have frequencies $\omega=\tilde{\Omega}_{l}$ such that $\beta-\tilde{\Omega}_{l}^{2}=0$. In this case Eq. (A.6) can be easily integrated:

$$
\Psi_{ \pm}=\chi_{ \pm} \int d r_{*} / \chi_{ \pm}^{2}=\chi_{ \pm}\left(C_{ \pm 1}+C_{ \pm 2} \int_{0}^{r} d r_{*} / \chi_{ \pm}^{2}\right),
$$

where $\chi_{ \pm}=\exp \left[ \pm \int W d r_{*}\right]$. The special relation between the two gravitational potentials extends to the Kerr geometry, where AS modes correspond to a vanishing Teukolsky-Starobinsky constant 22]. The nature of the boundary conditions at the Schwarzschild AS frequency is extremely subtle, and has been studied in detail by Maassen van den Brink [745]. Let us introduce some terminology [745]:

1) "standard" QNMs have outgoing-wave boundary conditions at both sides (that is, they are outgoing at infinity and "outgoing into the horizon");

2) total transmission modes from the left $\left(\mathrm{TTM}_{L}\right.$ 's) are incoming from the left (the $\mathrm{BH}$ horizon) and outgoing to the right (spatial infinity);

3) total transmission modes from the right $\left(\mathrm{TTM}_{R}\right.$ 's) are incoming from the right and outgoing to the left.

The Regge-Wheeler equation and the Zerilli equation must be treated on different footing at the AS frequency, since the supersymmetry transformation leading to the proof of isospectrality is singular there. It turns out that the Regge-Wheeler equation has no modes at all, while the Zerilli equation has both a QNM and a TTM $M_{L}$ at the $A S$ frequency 745 .

Numerical calculations of AS modes have yielded some puzzling results. Studying the Regge-Wheeler equation, Leaver 75 found a QNM very close (but not exactly at) the AS frequencies of Eq. (83). Namely, he found QNMs at frequencies $2 M \tilde{\Omega}_{2}^{\prime}=$ $0.000000-3.998000 i$ and $2 M \tilde{\Omega}_{3}^{\prime}=-0.000259-20.015653 i$. Similarly, in the extremal RN case one finds a QNM frequency very close to, but not exactly equal to, the AS frequency 241. Maassen van den Brink 745 speculated that the numerical calculations may be inaccurate and that no conclusion can be drawn on the coincidence of $\tilde{\Omega}_{l}$ and $\tilde{\Omega}_{l}^{\prime}$, "if the latter does exist at all".

An independent calculation was carried out by Andersson [746], who found that the Regge-Wheeler equation has pure imaginary $\mathrm{TTM}_{R}$ 's very close to $\tilde{\Omega}_{l}$ for $2 \leq l \leq 6$. He therefore suggested that the modes he found coincide with $\tilde{\Omega}_{l}$, which would then be a TTM. Maassen van den Brink 745] observed again that, if all figures in the computed modes are significant, the coincidence of TTM's and QNMs is not confirmed by this calculation, since $\tilde{\Omega}_{l}^{\prime}$ and $\tilde{\Omega}_{l}$ are numerically (slightly) different.

Onozawa 230] calculated the (TTM) AS mode for Kerr BHs, improving upon the accuracy of the Kerr AS frequencies computed in Ref. 142. He showed that the Kerr QNM with overtone $n=9$ tends to the AS frequency $\tilde{\Omega}_{l}$ (as defined by the TeukolskyStarobinsky identity) when $a \rightarrow 0$ and suggested that QNMs approaching $\tilde{\Omega}_{l}$ from the left and from the right may cancel at $a=0$, leaving only a special (TTM) mode. The situation concerning Kerr QNMs branching from the AS Schwarzchild mode is still far from clear. Using slow-rotation expansions of the perturbation equations, Maassen van den Brink [745] drew two basic conclusions on these modes. The first is that, for $a>0$, the so-called Kerr special modes are all TTM's (left or right, depending on the sign of $s$ ). The $\mathrm{TTM}_{R}$ 's should not survive as $a \rightarrow 0$, since they do not exist in the Schwarzschild limit. In particular, in this limit, the special Kerr mode becomes a $\mathrm{TTM}_{L}$ for $s=-2$; furthermore, the special mode and the $\mathrm{TTM}_{R}$ cancel each other for $s=2$. Studying the limit $a \rightarrow 0$ in detail, Maassen van den Brink reached a 
second conclusion: the Schwarzschild special frequency $\tilde{\Omega}_{l}$ should be a limit point for a multiplet of "standard" Kerr QNMs, which for small $a$ are well approximated by

$$
2 M \omega=-4 i-\frac{33078176}{700009} \frac{m a}{2 M}+\frac{3492608}{41177} i \frac{a^{2}}{4 M^{2}}+\mathcal{O}\left(m a^{2}\right)+\mathcal{O}\left(a^{4}\right)(\mathrm{A} .8)
$$

when $l=2$, and by his Eq. (7.33) when $l>2$. Numerical studies found QNMs close to the AS frequency, but not in agreement with this analytical prediction 231. It was suggested (note [46] in Ref. [745]) that QNMs corresponding to the AS frequency with $m>0$ may have one of the following three behaviors in the Schwarzschild limit: they may merge with those having $m<0$ at a frequency $\tilde{\Omega}_{l}^{\prime}$ such that $\left|\tilde{\Omega}_{l}^{\prime}\right|<\left|\tilde{\Omega}_{l}\right|$ (but $\left|\tilde{\Omega}_{l}^{\prime}\right|>\left|\tilde{\Omega}_{l}\right|$ for $l \geq 4$ ) and disappear, as suggested by Onozawa [230]; they may terminate at some (finite) small $a$; or, finally, they may disappear towards $\omega=-i \infty$. Recently another alternative was suggested [747, that in the Schwarzschild geometry a pair of "unconventional damped modes" should exist. For $l=2$ these modes were identified by a fitting procedure to be located on the unphysical sheet lying behind the branch cut (hence the name "unconventional") at $2 M \omega_{ \pm}=\mp 0.027+(0.0033-4) i$. An approximate analytical calculation confirmed the presence of these modes, yielding $2 M \omega_{+} \simeq-0.03248+(0.003436-4) i$, in reasonable agreement with the above prediction. If the prediction is true, an additional QNM multiplet should emerge near $\tilde{\Omega}_{l}$ as $a$ increases. This multiplet "may well be due to $\omega_{ \pm}$splitting (since spherical symmetry is broken) and moving through the negative imaginary axis as a is tuned" [747]. The emergence of such multiplets was shown in Ref. [231, but these do not seem to behave exactly as predicted [747.

Instability of naked singularities AS modes play an important role in the stability analysis of certain spacetimes containing naked singularities. An example of such a spacetime is the negative-mass Schwarzschild geometry, Eq. (3) with $L \rightarrow \infty$ and $M<0$. From the general solution (A.7) with $C_{-2}=0$ it follows that

$$
\Psi_{-}=C_{-1} \chi_{-}=C_{-1} r(6 M+(l-1)(l+2) r)^{-1} e^{-\frac{\lambda(\lambda+1)}{3 M} r_{*}},
$$

where $r_{*}=(r+2 M \log [-2 M+r])$, is a regular Zerilli or scalar-type gravitational mode in the entire spacetime with frequency $\omega=i \lambda(\lambda+1) /(3 M)$. Since $\omega_{I}>0$, the spacetime is unstable. This instability was first found numerically by Gleiser and Dotti [748 and recognized to be an "algebraically special instability" in Ref. [749]. In fact, the calculation above can be extended to (negative-mass) charged BHs in de Sitter spacetimes [749]. The AS mode is not unstable for negative-mass Kerr geometries, but numerical results show that both negative-mass and overspinning "Kerr" geometries are unstable [750, 751, 554, 555]. 


\section{Appendix B.}

\section{Highly damped modes of Kerr black holes}

Here we reproduce the formulas from Kao and Tomino [222, that allow one to compute analytically the highly damped modes of the Kerr spacetime, and which have been used to compare against numerical results in Fig. 10. Define $u_{1}=a\left(\lambda^{1 / 3}-\lambda^{-1 / 3}\right) / 2 \sqrt{3}$, $v_{1}=a\left(\lambda^{1 / 3}+\lambda^{-1 / 3}\right) / 2$ and $\lambda=3 \sqrt{3} M / a+\sqrt{1+27 M^{2} / a^{2}}$. Define also

$$
\begin{aligned}
f_{0}\left(r_{0}\right) & =i r_{0} \sqrt{3 u_{1}^{2}+v_{1}^{2}+2 i u_{1} v_{1}} E\left[\Upsilon_{0}\right]-\frac{i\left(r_{0}-u_{1}\right)\left(9 u_{1}^{2}+v_{1}^{2}\right)}{\sqrt{3 u_{1}^{2}+v_{1}^{2}+2 i u_{1} v_{1}}} K\left[\Upsilon_{0}\right] \\
& -\frac{i 2 r_{0}\left(3 u_{1}+i v_{1}\right)\left(r_{0}-u_{1}+i v_{1}\right)}{\sqrt{3 u_{1}^{2}+v_{1}^{2}+2 i u_{1} v_{1}}} \Pi\left[\Upsilon_{-1}, \Upsilon_{0}\right] \\
& -\frac{i\left(3 u_{1}+i v_{1}\right)\left(3 u_{1}^{2}-v_{1}^{2}-2 r_{0}^{2}\right)}{\sqrt{3 u_{1}^{2}+v_{1}^{2}+2 i u_{1} v_{1}}} \Pi\left[\frac{-2 i v_{1}}{3 u_{1}-i v_{1}}, \Upsilon_{0}\right],
\end{aligned}
$$

with $\Upsilon_{-1}=\frac{-2 i v_{1}\left(r_{0}+2 u_{1}\right)}{\left(r_{0}-u_{1}-i v_{1}\right)\left(3 u_{1}-i v_{1}\right)}, \Upsilon_{0}=\frac{4 i u_{1} v_{1}}{3 u_{1}^{2}+v_{1}^{2}+2 i u_{1} v_{1}}$ and

$$
\begin{aligned}
f_{m}\left(r_{0}\right)= & \frac{2}{r_{0} \sqrt{3 u_{1}^{2}+v_{1}^{2}-2 i u_{1} v_{1}}} \times\left\{F\left[\sin ^{-1} \Upsilon_{1}, \Upsilon_{2}\right]-K\left[\Upsilon_{2}\right]\right\} \\
- & \frac{4 u_{1}}{r_{0}\left(r_{0}+2 u_{1}\right) \sqrt{3 u_{1}^{2}+v_{1}^{2}-2 i u_{1} v_{1}}} \\
& \times\left\{\Pi\left[\Upsilon_{3}, \sin ^{-1} \Upsilon_{1}, \Upsilon_{2}\right]-\Pi\left[\Upsilon_{3}, \Upsilon_{2}\right]\right\} .
\end{aligned}
$$

Here $\Upsilon_{1} \equiv \sqrt{\frac{3 u_{1}^{2}+v_{1}^{2}-2 i u_{1} v_{1}}{3 u_{1}^{2}+v_{1}^{2}+2 i u_{1} v_{1}}}, \Upsilon_{2}=\frac{3 u_{1}^{2}+v_{1}^{2}+2 i u_{1} v_{1}}{3 u_{1}^{2}+v_{1}^{2}-2 i u_{1} v_{1}}$ and $\Upsilon_{3}=\frac{r_{0}\left(3 u_{1}-i v_{1}\right)}{\left(r_{0}+2 u_{1}\right)\left(u_{1}-i v_{1}\right)}$. The functions $E(m), E(\varphi, m), K(m), F(\varphi, m), \Pi(n, m)$ and $\Pi(n, \varphi, m)$ are the elliptical integrals:

$$
\begin{aligned}
& E(m) \quad=\int_{0}^{\frac{\pi}{2}} \sqrt{1-m \sin ^{2} \theta} d \theta, E(\varphi, m)=\int_{0}^{\varphi} \sqrt{1-m \sin ^{2} \theta} d \theta, \\
& K(m) \quad=\int_{0}^{\frac{\pi}{2}} \frac{1}{\sqrt{1-m \sin ^{2} \theta}} d \theta, F(\varphi, m)=\int_{0}^{\varphi} \frac{1}{\sqrt{1-m \sin ^{2} \theta}} d \theta, \\
& \Pi(n, m)=\int_{0}^{\frac{\pi}{2}}\left[1-n \sin ^{2} \theta\right]^{-1}\left[1-m \sin ^{2} \theta\right]^{-1 / 2} d \theta, \\
& \Pi(n, \varphi, m)=\int_{0}^{\varphi}\left[1-n \sin ^{2} \theta\right]^{-1}\left[1-m \sin ^{2} \theta\right]^{-1 / 2} d \theta .
\end{aligned}
$$

Because of the pole at $r=r_{0}$ and branch cuts, there is an ambiguity in Eqs. (B.1) and (B.2). The correct analytic expression is

$$
\delta_{0}=-2 i\left[\frac{f_{0}\left(r_{+}\right)-f_{0}\left(r_{-}\right)}{r_{+}-r_{-}}+\frac{i 4 \pi M r_{+}}{r_{+}-r_{-}} \Theta\left(3 u_{1} r_{+}-3 u_{1}^{2}-v_{1}^{2}\right)\right],
$$

where $\Theta(x)$ is the step function. The term with a step function is introduced to compensate the discontinuity caused by the term $\Pi\left[\frac{-2 i v_{1}\left(r_{+}+2 u_{1}\right)}{\left(r_{+}-u_{1}-i v_{1}\right)\left(3 u_{1}-i v_{1}\right)}, \frac{4 i u_{1} v_{1}}{3 u_{1}^{2}+v_{1}^{2}+2 i u_{1} v_{1}}\right]$. Similarly,

$$
\delta_{m}=4 i M a\left[r_{+} f_{m}\left(r_{+}, u_{1}, v_{1}\right)-r_{-} f_{m}\left(r_{-}, u_{1}, v_{1}\right)\right] /\left(r_{+}-r_{-}\right) .
$$

Note that here there is no discontinuity and we do not need to introduce any term with step function. The quantities $\varpi, \delta \omega_{I}$, defined in Eqs. (99) and (101), are related to $\delta_{0}, \delta_{m}$ via $\varpi=\delta_{m} / \delta_{0}, \delta \omega_{I}=2 \pi / \delta_{0}$. 


\section{Appendix C.}

\section{Supermassive black hole binary candidates}

At the moment of writing there are three plausible SMBH binary candidates at close separation. Due to the relevance of these observations for SMBH mergers and their rates (see e.g. [585]), here we briefly summarize the observations and their current interpretations.

SDSSJ092712.65+294344.0 - This quasar shows two sets of narrow emission lines, only one of which has associated broad lines, separated in velocity by $2650 \mathrm{~km} \mathrm{~s}^{-1}$, as well as additional emission and absorption lines at intermediate redshift. Komossa and collaborators 752 interpret the velocity separation between the two sets of lines as evidence for a recoiling $\mathrm{BH}$ with mass $M \approx 10^{8.8} M_{\odot}$. This large recoil speed can only be achieved if the final $\mathrm{BH}$ formed from the merger of two large-spin $\mathrm{BHs}$ in the so-called "superkick" configuration [753, 754, 755. The recoiling BH interpretation has been criticized by various authors. Bogdanovic et al. [756] propose a model where the blueshifted narrow lines originate from an accretion stream within the inner rim of the circumbinary disk of a massive $\mathrm{BH}$ binary with mass ratio $q \approx 0.1$ and mass $M_{2} \approx 10^{8} M_{\odot}$ for the secondary. Dotti et al. [757] also propose a model with a massive BH binary embedded in a circumbinary disk, where the blueshifted lines originate from gas swirling around the secondary $\mathrm{BH}$. The $\mathrm{BH}$ binary has mass ratio $q \approx 0.3, M_{1} \approx 2 \times 10^{9} M_{\odot}$, a semi-major axis $a \approx 0.34 \mathrm{pc}$ and an orbital period $P \approx 370 \mathrm{yrs}$. More detailed observations seem to favor the idea that the system represents the superposition of two AGN, rather than a recoiling SMBH [758]. Other interpretations suggest that the system is a more distant analog of NGC1275 [759], a large and a small galaxy interacting near the center of a rich cluster [760].

SDSSJ153636.22+044127.0 - This quasar shows two broad-line emission systems, separated in velocity by $3500 \mathrm{~km} / \mathrm{s}$, and unresolved absorption lines with intermediate velocity. Boroson and Lauer [584] interpret this quasar as a binary system of two SMBHs with masses $M_{1} \approx 10^{8.9} M_{\odot}$ and $M_{2} \approx 10^{7.3} M_{\odot}$ (hence $q \approx 40$ ) separated by $\sim 0.1 \mathrm{pc}$, with an orbital period of $\sim 100 \mathrm{yrs}$. Depending on unknown geometrical factors of the orbit, this system could coalesce either in $3 \times 10^{11}$ yrs or in $7 \times 10^{9} \mathrm{yrs}$. Several alternative interpretations of the observations have been proposed. Gaskell [761 points out that the blueshift/redshift of the broad emission lines in this system can be interpreted in terms of normal line emission from a disk in an AGN. Radio observations by Wrobel and Laor 762 identify two faint, spatially distinct radio sources within the quasar's optical localization region, and suggest that the system could consist of a quasar pair separated by $\sim 5 \mathrm{kpc}$. Chornock et al. 763 , 764 find a third broad emission component in the system, note a lack in velocity shift between the blueshifted and redshifted components, and argue that the system may be an unusual member of a class of AGNs known as "double-peaked emitters," rather than a SMBH binary or a quasar pair. Lauer and Boroson [765] remark that the lack of amplitude variations is unusual for a double-peaked emitter, and that longer temporal baselines are required to rule out the binary hypothesis.

OJ287 - This BL Lac object shows quasi-periodical optical outbursts at 12-year intervals, with two outburst peaks (separated by one to two years) per interval. Optical observations of this source date back to 1890. Valtonen and collaborators interpret the two outbursts as happening when a smaller $\mathrm{BH}$ pierces the accretion disk of the primary $\mathrm{BH}$, producing two impact flashes per period (see [766] for 
details of the model). A model with non-spinning BHs of mass $M_{1}=1.8 \times 10^{10} M_{\odot}$, $M_{2}=1 \times 10^{8} M_{\odot}$, semi-major axis $a \simeq 0.045$ pc and eccentricity $e \simeq 0.66$ was able to predict the date of the latest outburst. Remarkably, this model only reproduces the observations if gravitational radiation reaction is included [583. Indeed, if the interpretation is correct, the system would inspiral very quickly and merge in $\sim 10^{4}$ years. Alternative models attribute the observed behavior to (1) oscillations in the accretion disk or jet of a single $\mathrm{BH}$, or (2) variations of the accretion rate in a disk or a wobble of a jet in a binary BH (see [576, 583, for more details).

If the binary $\mathrm{BH}$ interpretation is correct, the orbital parameters of the two candidate BH binaries from Refs. [583, 584 may not be too dissimilar: in gravitational wave lingo, both systems would be IMRIs (intermediate mass ratio inspirals).

\section{Appendix D.}

\section{Black hole spin estimates}

Estimating BH masses is relatively easy because mass has a measurable effect even at large radii, where Newtonian gravity applies. Spin, on the contrary, is only measurable by looking at orbits close to the $\mathrm{BH}$, where relativistic effects are important. The reason is that the gravitational potential around a rotating object can be expanded as

$$
\Phi(r, \theta)=-(M / r)-q(M / r)^{3} P_{2}(\cos \theta)+\mathcal{O}\left(r^{-4}\right) .
$$

The parameter $q=Q / M^{3}$ is a measure of the mass quadrupole moment, and to lowest order it scales quadratically with the spins [767, 768]; for BHs, $Q=-M a^{2}$. Since the leading-order spin contribution scales like $(M / r)^{3}$, spin measurements must rely on observations of test particles on orbits with very small radii.

Luckily, we do have a chance to observe such orbits by looking at accreting gas close to the ISCO allowed by general relativity. All methods to measure spin using electromagnetic observations are based on variants of this idea, which is illustrated in the left panel of Fig. 15. There we show the variation of the ISCO frequency $M \Omega_{\mathrm{ISCO}}$ and of the light-ring frequency $M \Omega_{\mathrm{LR}}$ with the dimensionless spin parameter $a / M$ [160]. Positive values of $a / M$ correspond to corotating orbits, and negative values correspond to counterrotating orbits. The orbital frequency at the ISCO has a maximum when $a / M=1\left(R_{\mathrm{ISCO}}=M\right)$ and a minimum when $a / M=-1$ $\left(R_{\mathrm{ISCO}}=9 M\right)$; for a Schwarzschild $\mathrm{BH}, R_{\mathrm{ISCO}}=6 M$ and $M \Omega_{\mathrm{ISCO}}=6^{-3 / 2}$. The gas in an accretion disk spirals in through a sequence of quasi-circular orbits as it viscously loses angular momentum; when it reaches the ISCO, it accelerates radially and falls into the $\mathrm{BH}$, so the ISCO can be thought of as the inner edge of the accretion disk. The radiation efficiency $\eta$, also plotted in Fig. [15, yields the energy radiated by the accretion disk per unit accreted mass. In principle $\eta$ is determined by the binding energy of gas at the ISCO, which depends only on $a / M$.

Most spin estimates are based on the observation of electromagnetic emission from accreting gas close to the ISCO. Unfortunately, all of these estimates are to some degree model-dependent. Some corrections to the highly idealized scenario described above are discussed in a number of papers [618, 769, 770]. For example, Beckwith and Hawley [770. claim that an accurate modeling of magnetic fields would significantly displace the "inner edge" of the accretion disk from the standard general relativistic ISCO, and that this would lower the spin estimates inferred from Fe K $\alpha$ observations. More in general, systematic errors originate from our limited understanding of i) the 
physical processes producing the X-ray spectra * , and ii) accretion physics in strongfield gravity regions. Because of these uncertainties, different models are sometimes capable of fitting the observations while predicting different values of the spin (see e.g. Ref. 32 for examples). A discussion of accretion disk physics as applied to a study of the strong-field gravity region around BHs is outside the scope of this paper, and we refer the reader to the many excellent review articles on this topic $甘$.

Schematically, we can classify the main techniques used so far to estimate $\mathrm{BH}$ spins as belonging to four groups [8, 464, 32.

(a) Continuum spectroscopy of accretion disks has been applied to various stellar-mass $\mathrm{BH}$ candidates. This method was pioneered by Zhang et al. [618] to suggest that two galactic superluminal jet sources, GRO J1655-40 and GRS 1915+105, should harbor rapidly spinning BHs. Narayan, McClintock and collaborators have embarked in a program to estimate spins for about a dozen BH candidates 614, 619, 778, 779, 780, 616, 620, (but other groups are also very active 617, 781, 613, 782]). They also proposed that accretion can be used to provide hints of the presence of an event horizon for galactic stellar-mass BH binaries [32, 8] (but see [783] for criticism).

The basic idea is that when BHs have a large mass accretion rate the accreting gas tends to be optically thick, radiating approximately as a blackbody. In this spectral state (known as the "high soft state") the flux of radiation $F(R)$ emitted by the accretion disk can be computed 784 and used to obtain an effective temperature profile $T(r)=[F(R) / \sigma]^{1 / 4}$, where $\sigma$ is the Stefan-Boltzmann constant. By comparing the blackbody radiation with the spectral flux received at Earth one can estimate the quantity $R_{\text {in }} \cos \iota / D$, where $R_{\text {in }}$ is the inner edge of the accretion disk, $D$ is the distance to the source and $\iota$ the inclination angle. Unfortunately the method relies on independent estimates of $\iota$ and $D$. Perhaps the major weakness of the method consists in the fact that, in practice, the observed spectrum deviates significantly from a blackbody. These deviations are usually modeled by artificially increasing the temperature of the emitted radiation by a poorly known "spectral hardening factor", which is usually (and roughly) approximated by a constant $f_{\text {col }} \simeq 1.7$. Perhaps the most precise spin measurements to date using this technique regard the eclipsing $\mathrm{X}$ ray binary $\mathrm{M} 33 \mathrm{X}-7$, with a claimed value $a / M=0.77 \pm 0.05616$, and the first extragalactic X-ray binary LMC X-1, for which $a / M=0.90_{-0.09}^{+0.04}[620$.

(b) Spectroscopy of relativistically broadened $F e ~ K \alpha$ fluorescence lines has been proposed as a promising alternative to continuum fitting. This method originated from the discovery of a strong, broad spectral line in the X-ray spectrum of the Seyfert 1.2 galaxy MCG-6-30-15 [785. Brenneman and Reynolds 607] applied the method to XMM-Newton observations of this system, estimating the spin to be very near the maximal limit: $a / M=0.989_{-0.002}^{+0.009}$. In this case, the largest source of error comes from the unknown dependence of the line emissivity on the disk radius $R$, which is usually modeled as a power law $[8$. An analysis of systematic errors involved in the $\mathrm{Fe} \mathrm{K} \alpha$ measurements was carried out by Reynolds and Fabian [769]. Their

* The most pessimistic viewpoint on electromagnetic spin measurements is perhaps that of Miller and Turner: according to them, "with the uncertainty and debate on the physical processes that dominate the X-ray spectrum in this energy range [...] it is not currently possible to constrain the BH spin in a model-independent way" [771] (see also [772]).

\# Liedahl and Torres 773 focus on the basic general relativistic equations, scattering processes and atomic transitions. Reynolds and Nowak [774] discuss theoretical problems in the modeling of relativistic disk lines. Fabian and Miniutti 775 and Miller 776 review relativistic X-ray emission lines from the inner accretion disks around rotating BHs and observational prospects for constraining the spin history of SMBHs (see also 777, for a similar review focusing on stellar-mass BHs). 
main finding is that systematic errors can be significant for modest values of the spin, but they decrease for large spins, so large-spin measurements (such as the one of Ref. 607]) should be more reliable. The method has been applied to other AGN 786, 787, 788, 789 and even to stellar mass BH candidates 777. A very accurate measurement of $a / M=0.935 \pm 0.01$ (statistical) \pm 0.01 (systematic) has recently been claimed by Miller et al. 621] and Reis et al. 790, for the stellar-mass BH GX 339-4 in outburst. Ref. 612 discusses BH spin estimates for several different systems and their correlations with other physical properties of X-ray binaries.

(c) Quasi-periodic oscillations (QPOs) are likely to offer the most reliable spin measurements for accreting systems that harbor stellar-mass $\mathrm{BH}$ candidates, once the correct model is known. Unfortunately, at present there are several competing models to explain QPOs (see [464, 32, 791] for a discussion and further references), and models based on different physical assumptions typically yield very different spin estimates. For example, Figure 12 in Psaltis's review 32 shows that the diskoseismology model of Ref. 792 and the parametric resonance model of Ref. [793] give very different $\mathrm{BH}$ spin estimates in the case of GRO J1655-40. At the very least, an indication that QPOs are related to dynamical frequencies near the ISCO comes from the fact that the QPO frequencies roughly scale with the inverse of the BH masses, as they should in general relativity. A relatively model-independent lower limit on the spin can then be obtained for GRO J1655-40: for this system one gets a lower limit of $a / M \geq 0.25$ by requiring only that the observed $450 \mathrm{~Hz}$ oscillation frequency must be limited by the azimuthal frequency at the ISCO, a rather solid assumption 622 .

(d) Statistical methods based on the radiative efficiency of AGN. A rather general argument constrains, in principle, the average properties of the spin distribution in AGN. The general idea is suggested by a glance at the efficiency $\eta=L_{\text {acc }} / \dot{M} c^{2}$ (i.e., the energy radiated $L_{\text {acc }}$ per unit accreted mass) in the right panel of Figure 15. For a non-rotating $\mathrm{BH} \eta=1-(8 / 9)^{1 / 2} \simeq 0.057$, while for a maximally rotating Kerr $\mathrm{BH}$ $\eta \simeq 0.42$ is much larger. In a typical accretion system one can measure $L_{\text {acc }}$ (provided the distance is known), but not $\dot{M}$, so there is no way of knowing $\eta$ accurately enough to estimate $a / M$. However, by observing high-redshift AGN one can estimate the mean energy radiated by SMBHs per unit volume. By considering SMBHs in nearby galaxies we can estimate the mean mass in SMBHs per unit volume of the current universe. If we assume that SMBHs grow mostly by accretion, by dividing these two quantities we can get the average radiative efficiency of AGN, hence their average spin (this is a variant of the famous "Soltan argument" 609]).

Elvis et al. 626] and $\mathrm{Yu}$ and Tremaine 627 use observational data to infer that the mean efficiency $\eta \sim 0.10-0.15$ on average, and $\eta \sim 0.2$ (or larger) for the most massive systems. This is possible only if SMBHs have significant rotation. Their arguments have been supported by several later studies, some of which claim an average efficiency $\eta \sim 0.3-0.35$ for quasars at moderate redshift 628, 629, 630. However there is no general consensus on the interpretation of the data. Shankar et al. 631, Merloni and Heinz 632 and Daly 633 present arguments in favor of low average radiative efficiencies, hence low spins. Wang et al. 606] argue that the radiative efficiency has a strong cosmological evolution, decreasing from $\eta \approx 0.3$ at $z \approx 2$ to $\eta \approx 0.03$ at $z \approx 0$. The uncertainties in disentangling radiative efficiencies from quasar lifetimes were pointed out in Ref. 794. Daly 610] provides spin estimates for 19 powerful FRII radio sources and for 29 central dominant galaxies (CDGs). For the first class of sources the spins seem to decrease from near-extremal values at $z=2$ to $\approx 0.7$ at $z=0$, while for the (lower power) CDGs the estimated spins are in the 
range $0.1-0.8$. In conclusion, the jury is still out on the mean radiative efficiency (and mean spin) of quasars. The outcome of this debate is fundamental to constrain SMBH evolution models 482, 601, 795].

(e) Other methods. Besides the methods listed above, there are other avenues for measuring $\mathrm{BH}$ spin that hold promise for the future. One of these is polarimetry. The idea is to exploit the fact that, in general relativity, the plane of polarization of $\mathrm{BH}$ disk radiation changes with energy. This is a purely relativistic effect, absent in Newtonian gravity, and the magnitude of the change of the plane of polarization can give a direct measure of $a / M$ 796, 464. A second idea is based on Very Long Baseline Interferometry (VLBI) imaging of the silhouette of the SMBH in M87. This system is at a distance of $16 \mathrm{Mpc}$, much farther away than the SMBH at the galactic center; however, because of its larger mass of $\sim 3.4 \times 10^{9} M_{\odot}$, the apparent diameter of M87's horizon is $22 \mu \mathrm{as}$, about half as large as Sgr A*. Unlike Sgr A*, M87 exhibits a powerful radio jet, hence it holds promise for exploring the relation between the $\mathrm{BH}$ spin and the jet generation mechanism 797. A third, indirect way to constrain BH spins is based on energetic considerations. The AGN outburst in the MS0735.6+7421 cluster's central galaxy implies that its putative SMBH grew by about $1 / 3$ of its mass in the past $100 \mathrm{Myr}$, accreting matter at $\sim 3-5 M_{\odot} / \mathrm{yr}$, inconsistent with the Bondi mechanism. The energetics of the system could be explained instead by angular momentum released from a rapidly spinning $\mathrm{SMBH}$ with $M>10^{10} M_{\odot} 611$.

[1] M. Kac, Am. Math. Mon. 73, 1 (1966).

[2] E. S. C. Ching et al., Rev. Mod. Phys. 70, 1545 (1998).

[3] H.-P. Nollert and R. H. Price, J. Math. Phys. 40, 980 (1999), [gr-qc/9810074].

[4] K. D. Kokkotas and B. G. Schmidt, Living Rev. Rel. 2, 2 (1999), [gr-qc/9909058].

[5] H.-P. Nollert, Class. Quant. Grav. 16, R159 (1999).

[6] J. M. Maldacena, Adv. Theor. Math. Phys. 2, 231 (1998), [hep-th/9711200].

[7] D. T. Son and A. O. Starinets, Ann. Rev. Nucl. Part. Sci. 57, 95 (2007), [0704.0240].

[8] R. Narayan, New J. Phys. 7, 199 (2005), [gr-qc/0506078].

[9] F. Echeverria, Phys. Rev. D40, 3194 (1989).

[10] E. Berti, V. Cardoso and C. M. Will, Phys. Rev. D73, 064030 (2006), [gr-qc/0512160].

[11] E. Berti, J. Cardoso, V. Cardoso and M. Cavaglia, Phys. Rev. D76, 104044 (2007), [0707.1202].

[12] J. D. E. Creighton, Phys. Rev. D60, 022001 (1999), [gr-qc/9901084].

[13] TAMA, Y. Tsunesada, D. Tatsumi, N. Kanda and H. Nakano, Class. Quant. Grav. 22, S1129 (2005).

[14] F. Pretorius, Phys. Rev. Lett. 95, 121101 (2005), [gr-qc/0507014].

[15] M. Campanelli, C. O. Lousto, P. Marronetti and Y. Zlochower, Phys. Rev. Lett. 96, 111101 (2006), [gr-qc/0511048].

[16] J. G. Baker, J. Centrella, D.-I. Choi, M. Koppitz and J. van Meter, Phys. Rev. Lett. 96, 111102 (2006), [gr-qc/0511103].

[17] S. Hod, Phys. Rev. Lett. 81, 4293 (1998), [gr-qc/9812002].

[18] V. Ferrari and L. Gualtieri, Gen. Rel. Grav. 40, 945 (2008), [0709.0657].

[19] C. Cutler and K. S. Thorne, gr-qc/0204090.

[20] B. S. Sathyaprakash and B. F. Schutz, Living Rev. Rel. 12, 2 (2009), [0903.0338].

[21] K. S. Thorne, Rev. Mod. Phys. 52, 299 (1980).

[22] S. Chandrasekhar, The Mathematical Theory of Black Holes (Oxford University Press, New York, 1983).

[23] T. Nakamura, K. Oohara and Y. Kojima, Prog. Theor. Phys. Suppl. 90, 1 (1987).

[24] Y. Mino, M. Sasaki, M. Shibata, H. Tagoshi and T. Tanaka, Prog. Theor. Phys. Suppl. 128, 1 (1997), [gr-qc/9712057].

[25] V. P. Frolov and I. D. Novikov, Black hole physics: Basic concepts and new developments, Dordrecht, Netherlands: Kluwer Academic (1998) 770 p.

[26] M. Sasaki and H. Tagoshi, Living Rev. Rel. 6, 6 (2003), [gr-qc/0306120].

[27] A. Nagar and L. Rezzolla, Class. Quant. Grav. 22, R167 (2005), [gr-qc/0502064]. 
[28] E. Berti, V. Cardoso and C. M. Will, AIP Conf. Proc. 848, 687 (2006), [gr-qc/0601077].

[29] E. Berti and M. Campanelli, Living Rev. Rel. (2009).

[30] P. Amaro-Seoane et al., Class. Quant. Grav. 24, R113 (2007), [astro-ph/0703495].

[31] C. Barcelo, S. Liberati and M. Visser, Living Rev. Rel. 8, 12 (2005), [gr-qc/0505065].

[32] D. Psaltis, 0806.1531.

[33] C. M. Will, Living Rev. Rel. 9, 3 (2005), [gr-qc/0510072].

[34] R. Emparan and H. S. Reall, Living Rev. Rel. 11, 6 (2008), [0801.3471].

[35] O. Aharony, S. S. Gubser, J. M. Maldacena, H. Ooguri and Y. Oz, Phys. Rept. 323, 183 (2000), [hep-th/9905111].

[36] S. S. Gubser and A. Karch, 0901.0935.

[37] S. A. Hartnoll, 0903.3246.

[38] C. P. Herzog, 0904.1975.

[39] M. Heusler, Living Rev. Rel. 1, 6 (1998).

[40] S. W. Hawking and W. Israel, Cambridge, United Kingdom: Univ.Pr.(1979) 919p.

[41] E. S. C. Ching, P. T. Leung, W. M. Suen and K. Young, Phys. Rev. D52, 2118 (1995), [gr-qc/9507035].

[42] C. V. Vishveshwara, Nature 227, 936 (1970).

[43] M. Davis, R. Ruffini, W. H. Press and R. H. Price, Phys. Rev. Lett. 27, 1466 (1971).

[44] E. Berti et al., Phys. Rev. D76, 064034 (2007), [gr-qc/0703053].

[45] U. Sperhake, V. Cardoso, F. Pretorius, E. Berti and J. A. Gonzalez, Phys. Rev. Lett. 101, 161101 (2008), [0806.1738].

[46] L. Baiotti, B. Giacomazzo and L. Rezzolla, Phys. Rev. D78, 084033 (2008), [0804.0594].

[47] Webpage with Mathematica notebooks and numerical quasinormal mode Tables: http://www.phy.olemiss . edu/ berti/qnms.html http://gamow.ist.utl.pt/ vitor/ringdown.html.

[48] T. Regge and J. A. Wheeler, Phys. Rev. 108, 1063 (1957).

[49] E. Newman and R. Penrose, J. Math. Phys. 3, 566 (1962).

[50] R. P. Kerr, Phys. Rev. Lett. 11, 237 (1963).

[51] M. Schmidt, Nature 197, 1040 (1963).

[52] C. T. Bolton, Nature 240, 124 (1972).

[53] R. Ruffini and J. A. Wheeler, Physics Today 24, 30 (1971).

[54] J. A. Wheeler, The Physics Teacher 7, 1 (1969).

[55] J. A. Wheeler and K. Ford, New York, USA: Norton (1998) 380 p.

[56] F. J. Zerilli, Phys. Rev. Lett. 24, 737 (1970).

[57] F. J. Zerilli, Phys. Rev. D2, 2141 (1970).

[58] W. H. Press, Astrophys. J. L170, 105 (1971).

[59] C. J. Goebel, Astrophys. J. L172, 95 (1972).

[60] S. A. Teukolsky, Phys. Rev. Lett. 29, 1114 (1972).

[61] V. Moncrief, Ann. Phys. 88, 323 (1974).

[62] S. Chandrasekhar and S. Detweiler, Royal Society of London Proceedings Series A 344, 441 (1975).

[63] C. T. Cunningham, R. H. Price and V. Moncrief, Astrophys. J. 224, 643 (1978).

[64] C. T. Cunningham, R. H. Price and V. Moncrief, Astrophys. J. 230, 870 (1979).

[65] C. T. Cunningham, R. H. Price and V. Moncrief, Astrophys. J. 236, 674 (1980).

[66] U. H. Gerlach and U. K. Sengupta, Phys. Rev. D19, 2268 (1979).

[67] U. H. Gerlach and U. K. Sengupta, Phys. Rev. D22, 1300 (1980).

[68] J. York, James W., Phys. Rev. D28, 2929 (1983).

[69] B. Mashhoon, in Proceedings of the Third Marcel Grossmann Meeting on Recent Developments of General Relativity, edited by H. Ning, p. 599, Amsterdam, 1983, North-Holland.

[70] V. Ferrari and B. Mashhoon, Phys. Rev. D30, 295 (1984).

[71] R. F. Stark and T. Piran, Phys. Rev. Lett. 55, 891 (1985).

[72] L. Baiotti and L. Rezzolla, Phys. Rev. Lett. 97, 141101 (2006), [gr-qc/0608113].

[73] B. Mashhoon, Phys. Rev. D31, 290 (1985).

[74] B. F. Schutz and C. M. Will, Astrophys. J. L291, 33 (1985).

[75] E. W. Leaver, Proc. Roy. Soc. Lond. A402, 285 (1985).

[76] E. W. Leaver, J. Math. Phys. 27, 1238 (1986).

[77] E. W. Leaver, Phys. Rev. D34, 384 (1986).

[78] J. E. McClintock and R. A. Remillard, Astrophys. J. 308, 110 (1986).

[79] L. S. Finn, Phys. Rev. D46, 5236 (1992), [gr-qc/9209010].

[80] H.-P. Nollert and B. G. Schmidt, Phys. Rev. D45, 2617 (1992).

[81] N. Froeman, P. O. Froeman, N. Andersson and A. Hoekback, Phys. Rev. D45, 2609 (1992). 
[82] P. Anninos, D. Hobill, E. Seidel, L. Smarr and W.-M. Suen, Phys. Rev. Lett. 71, 2851 (1993), [gr-qc/9309016].

[83] A. Bachelot and A. Motet-Bachelot, Annales Poincare Phys. Theor. 59, 3 (1993).

[84] E. S. C. Ching, P. T. Leung, W. M. Suen and K. Young, Phys. Rev. Lett. 74, 2414 (1995), [gr-qc/9410044].

[85] R. J. Gleiser, C. O. Nicasio, R. H. Price and J. Pullin, Class. Quant. Grav. 13, L117 (1996), [gr-qc/9510049].

[86] R. J. Gleiser, C. O. Nicasio, R. H. Price and J. Pullin, Phys. Rev. Lett. 77, 4483 (1996), [gr-qc/9609022].

[87] S. S. Gubser, I. R. Klebanov and A. M. Polyakov, Phys. Lett. B428, 105 (1998), [hepth/9802109].

[88] E. Witten, Adv. Theor. Math. Phys. 2, 253 (1998), [hep-th/9802150].

[89] E. E. Flanagan and S. A. Hughes, Phys. Rev. D57, 4535 (1998), [gr-qc/9701039].

[90] H.-P. Nollert, Phys. Rev. D 47, 5253 (1993).

[91] L. Motl, Adv. Theor. Math. Phys. 6, 1135 (2003), [gr-qc/0212096].

[92] G. T. Horowitz and V. E. Hubeny, Phys. Rev. D62, 024027 (2000), [hep-th/9909056].

[93] M. Shibata and K. Uryu, Phys. Rev. D61, 064001 (2000), [gr-qc/9911058].

[94] D. Birmingham, I. Sachs and S. N. Solodukhin, Phys. Rev. Lett. 88, 151301 (2002), [hepth/0112055].

[95] V. Cardoso and J. P. S. Lemos, Phys. Rev. D63, 124015 (2001), [gr-qc/0101052].

[96] J. G. Baker, M. Campanelli and C. O. Lousto, Phys. Rev. D65, 044001 (2002), [gr-qc/0104063].

[97] O. Dreyer, Phys. Rev. Lett. 90, 081301 (2003), [gr-qc/0211076].

[98] D. T. Son and A. O. Starinets, JHEP 09, 042 (2002), [hep-th/0205051].

[99] A. O. Starinets, Phys. Rev. D66, 124013 (2002), [hep-th/0207133].

[100] A. Nunez and A. O. Starinets, Phys. Rev. D67, 124013 (2003), [hep-th/0302026].

[101] L. Motl and A. Neitzke, Adv. Theor. Math. Phys. 7, 307 (2003), [hep-th/0301173].

[102] H. Kodama and A. Ishibashi, Prog. Theor. Phys. 110, 701 (2003), [hep-th/0305147].

[103] A. Ishibashi and H. Kodama, Prog. Theor. Phys. 110, 901 (2003), [hep-th/0305185].

[104] H. Kodama and A. Ishibashi, Prog. Theor. Phys. 111, 29 (2004), [hep-th/0308128].

[105] L. Fidkowski, V. Hubeny, M. Kleban and S. Shenker, JHEP 02, 014 (2004), [hep-th/0306170].

[106] G. Festuccia and H. Liu, JHEP 04, 044 (2006), [hep-th/0506202].

[107] J. Natario and R. Schiappa, Adv. Theor. Math. Phys. 8, 1001 (2004), [hep-th/0411267].

[108] LIGO Scientific, 0711.3041.

[109] P. K. Kovtun and A. O. Starinets, Phys. Rev. D72, 086009 (2005), [hep-th/0506184].

[110] A. Parnachev and A. Starinets, JHEP 10, 027 (2005), [hep-th/0506144].

[111] P. Benincasa, A. Buchel and A. O. Starinets, Nucl. Phys. B733, 160 (2006), [hep-th/0507026].

[112] A. Buchel, Phys. Rev. D72, 106002 (2005), [hep-th/0509083].

[113] P. Benincasa and A. Buchel, JHEP 01, 103 (2006), [hep-th/0510041].

[114] A. O. Starinets, Phys. Lett. B670, 442 (2009), [0806.3797].

[115] N. Iqbal and H. Liu, Phys. Rev. D79, 025023 (2009), [0809.3808].

[116] S. Bhattacharyya, V. E. Hubeny, S. Minwalla and M. Rangamani, JHEP 02, 045 (2008), [0712.2456].

[117] T. Damour and M. Lilley, 0802.4169.

[118] I. R. Klebanov, hep-th/0009139.

[119] J. M. Maldacena, hep-th/0309246.

[120] M. J. Duff, hep-th/9912164.

[121] S. Cotsakis and G. W. Gibbons, Berlin, Germany: Springer (2000) 251 p.

[122] J. P. S. Lemos, Class. Quant. Grav. 12, 1081 (1995), [gr-qc/9407024].

[123] J. P. S. Lemos, Phys. Lett. B353, 46 (1995), [gr-qc/9404041].

[124] J. P. S. Lemos and V. T. Zanchin, Phys. Rev. D54, 3840 (1996), [hep-th/9511188].

[125] R. B. Mann, Class. Quant. Grav. 14, L109 (1997), [gr-qc/9607071].

[126] L. Vanzo, Phys. Rev. D56, 6475 (1997), [gr-qc/9705004].

[127] D. Birmingham, Class. Quant. Grav. 16, 1197 (1999), [hep-th/9808032].

[128] B. Carter, Commun. Math. Phys. 10, 280 (1968).

[129] F. Mellor and I. Moss, Phys. Rev. D41, 403 (1990).

[130] V. Cardoso and J. P. S. Lemos, Phys. Rev. D64, 084017 (2001), [gr-qc/0105103].

[131] V. Cardoso and J. P. S. Lemos, Class. Quant. Grav. 18, 5257 (2001), [gr-qc/0107098].

[132] R. Ruffini, Black Holes: les Astres Occlus (Gordon and Breach Science Publishers, New York, 1973).

[133] A. R. Edmonds, Angular momentum in quantum mechanics (Princeton University Press, Princeton, 1996). 
[134] L. C. B. Crispino, A. Higuchi and G. E. A. Matsas, Phys. Rev. D63, 124008 (2001), [grqc/0011070].

[135] J. Mathews, J. Soc. Ind. Appl. Math. 10, 768 (1962).

[136] C. V. Vishveshwara, Phys. Rev. D1, 2870 (1970).

[137] L. A. Edelstein and C. V. Vishveshwara, Phys. Rev. D1, 3514 (1970).

[138] N. Sago, H. Nakano and M. Sasaki, Phys. Rev. D67, 104017 (2003), [gr-qc/0208060].

[139] O. Sarbach and M. Tiglio, Phys. Rev. D64, 084016 (2001), [gr-qc/0104061].

[140] K. Martel and E. Poisson, Phys. Rev. D71, 104003 (2005), [gr-qc/0502028].

[141] S. Chandrasekhar, Proc. R. Soc. Lond. A343, 289 (1975).

[142] S. Chandrasekhar, Proc. R. Soc. Lond. A392, 1 (1984).

[143] D. R. Brill and J. A. Wheeler, Rev. Mod. Phys. 29, 465 (1957).

[144] D. N. Page, Phys. Rev. D13, 198 (1976).

[145] W. G. Unruh, Phys. Rev. D14, 3251 (1976).

[146] G. F. Torres del Castillo, J. Math. Phys. 30, 446 (1989).

[147] H. T. Cho, Phys. Rev. D68, 024003 (2003), [gr-qc/0303078].

[148] D. R. Brill, P. L. Chrzanowski, C. Martin Pereira, E. D. Fackerell and J. R. Ipser, Phys. Rev. D5, 1913 (1972).

[149] S. A. Teukolsky, Astrophys. J. 185, 635 (1973).

[150] R. A. Breuer, editor, Gravitational perturbation theory and synchrotron radiation, Lecture Notes in Physics, Berlin Springer Verlag Vol. 44 (, 1975).

[151] R. M. Wald, General Relativity (University of Chicago Press, Chicago, 1984).

[152] J. N. Goldberg, A. J. Macfarlane, E. T. Newman, F. Rohrlich and E. C. G. Sudarshan, J. Math. Phys. 8, 2155 (1967).

[153] E. Berti, V. Cardoso and M. Casals, Phys. Rev. D73, 024013 (2006), [gr-qc/0511111].

[154] P. L. Chrzanowski, Phys. Rev. D11, 2042 (1975).

[155] R. M. Wald, Phys. Rev. Lett. 41, 203 (1978).

[156] L. S. Kegeles and J. M. Cohen, Phys. Rev. D19, 1641 (1979).

[157] C. O. Lousto and B. F. Whiting, Phys. Rev. D66, 024026 (2002), [gr-qc/0203061].

[158] B. F. Whiting and L. R. Price, Class. Quant. Grav. 22, S589 (2005).

[159] N. Yunes and J. A. Gonzalez, Phys. Rev. D73, 024010 (2006), [gr-qc/0510076].

[160] J. M. Bardeen, W. H. Press and S. A. Teukolsky, Astrophys. J. 178, 347 (1972).

[161] N. Andersson, Phys. Rev. D55, 468 (1997), [gr-qc/9607064].

[162] I. G. Moss and J. P. Norman, Class. Quant. Grav. 19, 2323 (2002), [gr-qc/0201016].

[163] G. Michalogiorgakis and S. S. Pufu, JHEP 02, 023 (2007), [hep-th/0612065].

[164] I. Bakas, JHEP 01, 003 (2009), [0809.4852].

[165] I. Bakas, Class. Quant. Grav. 26, 065013 (2009), [0812.0152].

[166] N. Andersson, Phys. Rev. D51, 353 (1995).

[167] E. Berti and V. Cardoso, Phys. Rev. D74, 104020 (2006), [gr-qc/0605118].

[168] P. M. Morse and H. Feshbach, Methods of Theoretical Physics (McGraw-Hill, New York, 1953).

[169] H.-P. Nollert, Characteristic Oscillations of Black Holes and Neutron Stars: From Mathematical Background to Astrophysical Applications (, 2000), Habilitationsschrift Der Fakultat fur Physik der Eberhard-Karls-Universitat Tubingen.

[170] B. P. Jensen and P. Candelas, Phys. Rev. D33, 1590 (1986).

[171] R. H. Price, Phys. Rev. D5, 2419 (1972).

[172] M. Casals, S. R. Dolan, A. C. Ottewill and B. Wardell, 0903.0395.

[173] Y. Sun and R. H. Price, Phys. Rev. D38, 1040 (1988).

[174] A. Buonanno et al., 0902.0790.

[175] T. Damour and A. Nagar, 0902.0136.

[176] T. Damour and A. Nagar, Phys. Rev. D76, 064028 (2007), [0705.2519].

[177] I. Amado, C. Hoyos-Badajoz, K. Landsteiner and S. Montero, JHEP, 133 (2008), [0805.2570].

[178] I. Amado, C. Hoyos-Badajoz, K. Landsteiner and S. Montero, Phys. Rev. D77, 065004 (2008), [0710.4458].

[179] Pöschl, G. and Teller, E., Z. Phys. 83, 143 (1933).

[180] P. P. Fiziev, Class. Quant. Grav. 23, 2447 (2006), [gr-qc/0509123].

[181] P. P. Fiziev, 0902.1277.

[182] R. S. Borissov and P. P. Fiziev, 0903.3617.

[183] P. P. Fiziev, 0906.5108.

[184] M. Banados, C. Teitelboim and J. Zanelli, Phys. Rev. Lett. 69, 1849 (1992), [hep-th/9204099].

[185] I. Sachs and S. N. Solodukhin, JHEP 08, 003 (2008), [0806.1788].

[186] I. Sachs, JHEP 09, 073 (2008), [0807.1844].

[187] M. Abramowitz and I. A. Stegun, Handbook of Mathematical Functions with Formulas, 
Graphs, and Mathematical Tables (Dover, New York, 1972).

[188] S. Iyer and C. M. Will, Phys. Rev. D35, 3621 (1987).

[189] S. Iyer, Phys. Rev. D35, 3632 (1987).

[190] A. S. Barreto and M. Zworski, Math. Res. Lett. 4, 103 (1997).

[191] V. Cardoso and J. P. S. Lemos, Phys. Rev. D67, 084020 (2003), [gr-qc/0301078].

[192] A. Lopez-Ortega, Gen. Rel. Grav. 39, 1011 (2007), [0704.2468].

[193] C. P. Burgess and C. A. Lutken, Phys. Lett. B153, 137 (1985).

[194] V. Cardoso, R. Konoplya and J. P. S. Lemos, Phys. Rev. D68, 044024 (2003), [gr-qc/0305037].

[195] A. Lopez-Ortega, Gen. Rel. Grav. 38, 1565 (2006), [gr-qc/0605027].

[196] V. Cardoso, O. J. C. Dias and J. P. S. Lemos, Phys. Rev. D70, 024002 (2004), [hepth/0401192].

[197] C. Molina, Phys. Rev. D68, 064007 (2003), [gr-qc/0304053].

[198] L. Vanzo and S. Zerbini, Phys. Rev. D70, 044030 (2004), [hep-th/0402103].

[199] H. Narai, Sci. Rep. Tohoku Univ. 34, 160 (1950).

[200] H. Narai, Rep. Tohoku Univ. 35, 62 (1951).

[201] M. Caldarelli, L. Vanzo and S. Zerbini, hep-th/0008136.

[202] I. Ichinose and Y. Satoh, Nucl. Phys. B447, 340 (1995), [hep-th/9412144].

[203] J. D. Brown, Singapore, Singapore: World Scientific (1988) 152p.

[204] V. Cardoso, O. J. C. Dias and J. P. S. Lemos, Phys. Rev. D67, 064026 (2003), [hepth/0212168].

[205] Y. Decanini and A. Folacci, Phys. Rev. D79, 044021 (2009), [0901.1642].

[206] J. M. Stewart, Proc. R. Soc. London A424, 239 (1989).

[207] Y. Decanini, A. Folacci and B. Jensen, Phys. Rev. D67, 124017 (2003), [gr-qc/0212093].

[208] V. Cardoso, A. S. Miranda, E. Berti, H. Witek and V. T. Zanchin, Phys. Rev. D79, 064016 (2009), [0812.1806].

[209] Y. Decanini and A. Folacci, 0906.2601.

[210] S. Deser, R. Jackiw and S. Templeton, Ann. Phys. 140, 372 (1982).

[211] S. Deser, R. Jackiw and S. Templeton, Phys. Rev. Lett. 48, 975 (1982).

[212] R. Aros, C. Martinez, R. Troncoso and J. Zanelli, Phys. Rev. D67, 044014 (2003), [hepth/0211024].

[213] D. Birmingham and S. Mokhtari, Phys. Rev. D74, 084026 (2006), [hep-th/0609028].

[214] A. Ben-Menahem and S. J. Singh, Seismic waves anf sources (Dover, New York, 2000).

[215] C. M. Bender and S. A. Orszag, Advanced Mathematical Methods for Scientists and Engineers (McGraw-Hill, New York, 1978).

[216] R. A. Konoplya, Phys. Rev. D68, 024018 (2003), [gr-qc/0303052].

[217] S. R. Dolan and A. C. Ottewill, private communication.

[218] N. Froeman and P. O. Froeman, JWKB Approximation: Contributions to the Theory (Amsterdam, North-Holland Publishing Company, 1965).

[219] N. Andersson and C. J. Howls, Class. Quant. Grav. 21, 1623 (2004), [gr-qc/0307020].

[220] H.-P. Nollert, Phys. Rev. D47, 5253 (1993).

[221] U. Keshet and A. Neitzke, Phys. Rev. D78, 044006 (2008), [0709.1532].

[222] H.-c. Kao and D. Tomino, Phys. Rev. D77, 127503 (2008), [0801.4195].

[223] V. Cardoso, J. Natario and R. Schiappa, J. Math. Phys. 45, 4698 (2004), [hep-th/0403132].

[224] E. Berti and K. D. Kokkotas, Phys. Rev. D68, 044027 (2003), [hep-th/0303029].

[225] E. Berti, V. Cardoso and S. Yoshida, Phys. Rev. D69, 124018 (2004), [gr-qc/0401052].

[226] G. B. Arfken and H. J. Weber, Mathematical Methods for Physicists (Academic Press, New York, 1995).

[227] E. Berti, V. Cardoso and P. Pani, 0903.5311.

[228] S. Chandrasekhar and V. Ferrari, Proc. Roy. Soc. Lond. A434, 449 (1991).

[229] S. Chandrasekhar and V. Ferrari, Proc. Roy. Soc. Lond. A437, 133 (1992).

[230] H. Onozawa, Phys. Rev. D55, 3593 (1997), [gr-qc/9610048].

[231] E. Berti, V. Cardoso, K. D. Kokkotas and H. Onozawa, Phys. Rev. D68, 124018 (2003), [hep-th/0307013].

[232] E. W. Leaver, Phys. Rev. D41, 2986 (1990).

[233] E. Berti and K. D. Kokkotas, Phys. Rev. D71, 124008 (2005), [gr-qc/0502065].

[234] V. Cardoso, J. P. S. Lemos and S. Yoshida, Phys. Rev. D69, 044004 (2004), [gr-qc/0309112].

[235] V. Cardoso, J. P. S. Lemos and S. Yoshida, JHEP 12, 041 (2003), [hep-th/0311260].

[236] V. Cardoso, G. Siopsis and S. Yoshida, Phys. Rev. D71, 024019 (2005), [hep-th/0412138].

[237] Y. Morisawa and D. Ida, Phys. Rev. D71, 044022 (2005), [gr-qc/0412070].

[238] S. Yoshida and T. Futamase, Phys. Rev. D69, 064025 (2004), [gr-qc/0308077].

[239] R. A. Konoplya and A. Zhidenko, JHEP 06, 037 (2004), [hep-th/0402080]. 
[240] V. Cardoso, J. P. S. Lemos and S. Yoshida, Phys. Rev. D70, 124032 (2004), [gr-qc/0410107].

[241] E. Berti, gr-qc/0411025.

[242] H. T. Cho and Y. C. Lin, Class. Quant. Grav. 22, 775 (2005), [gr-qc/0411090].

[243] A. Neitzke, hep-th/0304080.

[244] A. Maassen van den Brink, J. Math. Phys. 45, 327 (2004), [gr-qc/0303095].

[245] S. Musiri and G. Siopsis, Class. Quant. Grav. 20, L285 (2003), [hep-th/0308168].

[246] S. Musiri and G. Siopsis, hep-th/0610170.

[247] S. Musiri and G. Siopsis, Phys. Lett. B650, 279 (2007).

[248] J.-l. Jing, Phys. Rev. D71, 124006 (2005), [gr-qc/0502023].

[249] T. Padmanabhan, Class. Quant. Grav. 21, L1 (2004), [gr-qc/0310027].

[250] H. Onozawa, T. Mishima, T. Okamura and H. Ishihara, Phys. Rev. D53, 7033 (1996), [grqc/9603021].

[251] D. L. Gunter, Phil. Trans. Roy. Soc. Lond. A296, 497 (1980).

[252] K. D. Kokkotas and B. F. Schutz, Phys. Rev. D37, 3378 (1988).

[253] N. Andersson, Royal Society of London Proceedings Series A 442, 427 (1993).

[254] N. Andersson and H. Onozawa, Phys. Rev. D54, 7470 (1996), [gr-qc/9607054].

[255] J. F. Chang and Y. G. Shen, Int. J. Theor. Phys. 46, 1570 (2007).

[256] J.-l. Jing and Q.-y. Pan, Nucl. Phys. B728, 109 (2005), [gr-qc/0506098].

[257] S. K. Chakrabarti, 0809.1004.

[258] H. T. Cho, Phys. Rev. D73, 024019 (2006), [gr-qc/0512052].

[259] H. Onozawa, T. Okamura, T. Mishima and H. Ishihara, Phys. Rev. D55, 4529 (1997), [grqc/9606086].

[260] T. Okamura, Phys. Rev. D56, 4927 (1997), [gr-qc/9703050].

[261] R. Kallosh, J. Rahmfeld and W. K. Wong, Phys. Rev. D57, 1063 (1998), [hep-th/9706048].

[262] S. Detweiler, Astrophys. J. 239, 292 (1980).

[263] K. Glampedakis and N. Andersson, Phys. Rev. D64, 104021 (2001), [gr-qc/0103054].

[264] V. Cardoso, Phys. Rev. D70, 127502 (2004), [gr-qc/0411048].

[265] S. Hod, Phys. Rev. D78, 084035 (2008), [0811.3806].

[266] C. L. Fryer, D. E. Holz and S. A. Hughes, Astrophys. J. 565, 430 (2002), [astro-ph/0106113].

[267] S. Hod and U. Keshet, Class. Quant. Grav. 22, L71 (2005), [gr-qc/0505112].

[268] U. Keshet and S. Hod, Phys. Rev. D76, 061501 (2007), [0705.1179].

[269] D. N. Page, Phys. Rev. D14, 1509 (1976).

[270] K. D. Kokkotas, Nuovo Cim. B108, 991 (1993).

[271] R. A. Konoplya, Phys. Rev. D68, 124017 (2003), [hep-th/0309030].

[272] E. Berti, M. Cavaglia and L. Gualtieri, Phys. Rev. D69, 124011 (2004), [hep-th/0309203].

[273] A. Rostworowski, Acta Phys. Polon. B38, 81 (2007), [gr-qc/0606110].

[274] R. A. Konoplya and A. Zhidenko, Nucl. Phys. B777, 182 (2007), [hep-th/0703231].

[275] D. Birmingham, Phys. Lett. B569, 199 (2003), [hep-th/0306004].

[276] G. Kunstatter, Phys. Rev. Lett. 90, 161301 (2003), [gr-qc/0212014].

[277] A. J. M. Medved, D. Martin and M. Visser, Class. Quant. Grav. 21, 1393 (2004), [grqc/0310009].

[278] A. J. M. Medved, D. Martin and M. Visser, Class. Quant. Grav. 21, 2393 (2004), [grqc/0310097].

[279] S. Das and S. Shankaranarayanan, Class. Quant. Grav. 22, L7 (2005), [hep-th/0410209].

[280] J. Kettner, G. Kunstatter and A. J. M. Medved, Class. Quant. Grav. 21, 5317 (2004), [grqc/0408042].

[281] R. G. Daghigh and G. Kunstatter, Class. Quant. Grav. 22, 4113 (2005), [gr-qc/0505044].

[282] R. G. Daghigh, G. Kunstatter and J. Ziprick, Class. Quant. Grav. 24, 1981 (2007), [grqc/0611139].

[283] R. Gregory and R. Laflamme, Phys. Rev. Lett. 70, 2837 (1993), [hep-th/9301052].

[284] R. Gregory and R. Laflamme, Nucl. Phys. B428, 399 (1994), [hep-th/9404071].

[285] H. Kudoh, Phys. Rev. D73, 104034 (2006), [hep-th/0602001].

[286] H. Ishihara et al., Phys. Rev. D77, 084019 (2008), [0802.0655].

[287] P. Kanti, Int. J. Mod. Phys. A19, 4899 (2004), [hep-ph/0402168].

[288] D. Ida, K.-y. Oda and S. C. Park, Phys. Rev. D67, 064025 (2003), [hep-th/0212108].

[289] S. S. Seahra, C. Clarkson and R. Maartens, Phys. Rev. Lett. 94, 121302 (2005), [grqc/0408032].

[290] H. K. Kunduri, J. Lucietti and H. S. Reall, Phys. Rev. D74, 084021 (2006), [hep-th/0606076].

[291] K. Murata and J. Soda, Class. Quant. Grav. 25, 035006 (2008), [0710.0221].

[292] H. Kodama, R. A. Konoplya and A. Zhidenko, Phys. Rev. D79, 044003 (2009), [0812.0445].

[293] H. Kodama, R. A. Konoplya and A. Zhidenko, 0904.2154. 
[294] S. W. Hawking and D. N. Page, Commun. Math. Phys. 87, 577 (1983).

[295] E. Witten, Adv. Theor. Math. Phys. 2, 505 (1998), [hep-th/9803131].

[296] A. Chamblin, R. Emparan, C. V. Johnson and R. C. Myers, Phys. Rev. D60, 064018 (1999), [hep-th/9902170].

[297] D. Yamada and L. G. Yaffe, JHEP 09, 027 (2006), [hep-th/0602074].

[298] C. T. Asplund and D. Berenstein, Phys. Lett. B673, 264 (2009), [0809.0712].

[299] R. M. Wald, J. Math. Phys. 21, 2802 (1980).

[300] A. Ishibashi and R. M. Wald, Class. Quant. Grav. 20, 3815 (2003), [gr-qc/0305012].

[301] A. Ishibashi and R. M. Wald, Class. Quant. Grav. 21, 2981 (2004), [hep-th/0402184].

[302] C. P. Herzog and D. T. Son, JHEP 03, 046 (2003), [hep-th/0212072].

[303] K. Skenderis and B. C. van Rees, Phys. Rev. Lett. 101, 081601 (2008), [0805.0150].

[304] K. Skenderis and B. C. van Rees, 0812.2909.

[305] J. S. F. Chan and R. B. Mann, Phys. Rev. D55, 7546 (1997), [gr-qc/9612026].

[306] J. S. F. Chan and R. B. Mann, Phys. Rev. D59, 064025 (1999).

[307] S. Kalyana Rama and B. Sathiapalan, Mod. Phys. Lett. A14, 2635 (1999), [hep-th/9905219].

[308] U. H. Danielsson, E. Keski-Vakkuri and M. Kruczenski, Nucl. Phys. B563, 279 (1999), [hepth/9905227].

[309] U. H. Danielsson, E. Keski-Vakkuri and M. Kruczenski, JHEP 02, 039 (2000), [hepth/9912209].

[310] R. A. Konoplya, Phys. Rev. D66, 044009 (2002), [hep-th/0205142].

[311] E. Berti and K. D. Kokkotas, Phys. Rev. D67, 064020 (2003), [gr-qc/0301052].

[312] S. Musiri and G. Siopsis, Phys. Lett. B563, 102 (2003), [hep-th/0301081].

[313] S. Musiri and G. Siopsis, Phys. Lett. B576, 309 (2003), [hep-th/0308196].

[314] S. Musiri, S. Ness and G. Siopsis, Phys. Rev. D73, 064001 (2006), [hep-th/0511113].

[315] A. S. Miranda and V. T. Zanchin, Phys. Rev. D73, 064034 (2006), [gr-qc/0510066].

[316] J. J. Friess, S. S. Gubser, G. Michalogiorgakis and S. S. Pufu, JHEP 04, 080 (2007), [hepth/0611005].

[317] T. R. Govindarajan and V. Suneeta, Class. Quant. Grav. 18, 265 (2001), [gr-qc/0007084].

[318] J.-M. Zhu, B. Wang and E. Abdalla, Phys. Rev. D63, 124004 (2001), [hep-th/0101133].

[319] V. Cardoso and J. P. S. Lemos, Phys. Rev. D66, 064006 (2002), [hep-th/0206084].

[320] B. Wang, E. Abdalla and R. B. Mann, Phys. Rev. D65, 084006 (2002), [hep-th/0107243].

[321] G. Siopsis, Phys. Lett. B590, 105 (2004), [hep-th/0402083].

[322] G. Siopsis, Nucl. Phys. B715, 483 (2005), [hep-th/0407157].

[323] A. Lopez-Ortega, Gen. Rel. Grav. 38, 1747 (2006), [gr-qc/0605034].

[324] G. Siopsis, JHEP 05, 042 (2007), [hep-th/0702079].

[325] M. Giammatteo and J.-l. Jing, Phys. Rev. D71, 024007 (2005), [gr-qc/0403030].

[326] J.-l. Jing, gr-qc/0502010.

[327] A. S. Miranda, J. Morgan and V. T. Zanchin, JHEP 11, 030 (2008), [0809.0297].

[328] Y. S. Myung, Y.-W. Kim and Y.-J. Park, Eur. Phys. J. C58, 617 (2008), [0809.1933].

[329] M. Natsuume and T. Okamura, Phys. Rev. D77, 066014 (2008), [0712.2916].

[330] M. Natsuume and T. Okamura, Prog. Theor. Phys. 120, 1217 (2008), [0801.1797].

[331] G. Festuccia and H. Liu, 0811.1033.

[332] J. Grain and A. Barrau, Nucl. Phys. B742, 253 (2006), [hep-th/0603042].

[333] V. Cardoso and O. J. C. Dias, Phys. Rev. D70, 084011 (2004), [hep-th/0405006].

[334] V. Cardoso, O. J. C. Dias and S. Yoshida, Phys. Rev. D74, 044008 (2006), [hep-th/0607162].

[335] R. G. Daghigh and M. D. Green, 0808.1596.

[336] I. Amado and C. Hoyos-Badajoz, JHEP 09, 118 (2008), [0807.2337].

[337] J. Morgan, V. Cardoso, A. S. Miranda, C. Molina and V. T. Zanchin, 0906.0064.

[338] B. Wang, C.-Y. Lin and E. Abdalla, Phys. Lett. B481, 79 (2000), [hep-th/0003295].

[339] B. Wang, C. Molina and E. Abdalla, Phys. Rev. D63, 084001 (2001), [hep-th/0005143].

[340] R. A. Konoplya, Phys. Rev. D66, 084007 (2002), [gr-qc/0207028].

[341] J.-l. Jing and Q.-y. Pan, Phys. Rev. D71, 124011 (2005), [gr-qc/0502011].

[342] Y. Zhang and J.-L. Jing, Int. J. Mod. Phys. D15, 905 (2006).

[343] R. A. Konoplya and A. Zhidenko, Phys. Rev. D78, 104017 (2008), [0809.2048].

[344] B. Wang, C.-Y. Lin and C. Molina, Phys. Rev. D70, 064025 (2004), [hep-th/0407024].

[345] C. M. Chambers and I. G. Moss, Class. Quant. Grav. 11, 1035 (1994), [gr-qc/9404015].

[346] M. Giammatteo and I. G. Moss, Class. Quant. Grav. 22, 1803 (2005), [gr-qc/0502046].

[347] V. Cardoso, O. J. C. Dias, J. P. S. Lemos and S. Yoshida, Phys. Rev. D70, 044039 (2004), [hep-th/0404096].

[348] H. Kodama, Prog. Theor. Phys. Suppl. 172, 11 (2008), [0711.4184].

[349] A. N. Aliev and O. Delice, Phys. Rev. D79, 024013 (2009), [0808.0280]. 
[350] K. Murata, 0812.0718.

[351] W. H. Press and S. A. Teukolsky, Nature 238, 211 (1972).

[352] P. R. Brady, C. M. Chambers, W. G. Laarakkers and E. Poisson, Phys. Rev. D60, 064003 (1999), [gr-qc/9902010].

[353] E. Abdalla, C. Molina and A. Saa, gr-qc/0309078.

[354] V. Suneeta, Phys. Rev. D68, 024020 (2003), [gr-qc/0303114].

[355] J.-l. Jing, Phys. Rev. D69, 084009 (2004), [gr-qc/0312079].

[356] J.-L. Jing and S.-B. Chen, Chin. Phys. 14, 683 (2005).

[357] A. Zhidenko, Class. Quant. Grav. 21, 273 (2004), [gr-qc/0307012].

[358] T. R. Choudhury and T. Padmanabhan, Phys. Rev. D69, 064033 (2004), [gr-qc/0311064].

[359] R. A. Konoplya and A. Zhidenko, Phys. Rev. D76, 084018 (2007), [0707.1890].

[360] F. Moura and R. Schiappa, Class. Quant. Grav. 24, 361 (2007), [hep-th/0605001].

[361] J. T. Wheeler, Nucl. Phys. B268, 737 (1986).

[362] J. T. Wheeler, Nucl. Phys. B273, 732 (1986).

[363] D. G. Boulware and S. Deser, Phys. Rev. Lett. 55, 2656 (1985).

[364] P. Kanti, N. E. Mavromatos, J. Rizos, K. Tamvakis and E. Winstanley, Phys. Rev. D54, 5049 (1996), [hep-th/9511071].

[365] T. Torii, H. Yajima and K.-i. Maeda, Phys. Rev. D55, 739 (1997), [gr-qc/9606034].

[366] S. O. Alexeev and M. V. Pomazanov, Phys. Rev. D55, 2110 (1997), [hep-th/9605106].

[367] P. Pani and V. Cardoso, 0902.1569.

[368] Z.-K. Guo, N. Ohta and T. Torii, Prog. Theor. Phys. 120, 581 (2008), [0806.2481].

[369] Z.-K. Guo, N. Ohta and T. Torii, Prog. Theor. Phys. 121, 253 (2009), [0811.3068].

[370] N. Ohta and T. Torii, 0902.4072.

[371] G. Dotti and R. J. Gleiser, Class. Quant. Grav. 22, L1 (2005), [gr-qc/0409005].

[372] G. Dotti and R. J. Gleiser, Phys. Rev. D72, 044018 (2005), [gr-qc/0503117].

[373] R. J. Gleiser and G. Dotti, Phys. Rev. D72, 124002 (2005), [gr-qc/0510069].

[374] T. Takahashi and J. Soda, 0902.2921.

[375] P. Kanti, N. E. Mavromatos, J. Rizos, K. Tamvakis and E. Winstanley, Phys. Rev. D57, 6255 (1998), [hep-th/9703192].

[376] B. R. Iyer, S. Iyer and C. V. Vishveshwara, Class. Quant. Grav. 6, 1627 (1989).

[377] E. Abdalla, R. A. Konoplya and C. Molina, Phys. Rev. D72, 084006 (2005), [hep-th/0507100].

[378] R. Konoplya, Phys. Rev. D71, 024038 (2005), [hep-th/0410057].

[379] S. K. Chakrabarti, Gen. Rel. Grav. 39, 567 (2007), [hep-th/0603123].

[380] R. A. Konoplya and A. Zhidenko, Phys. Rev. D77, 104004 (2008), [0802.0267].

[381] N. Arkani-Hamed, S. Dimopoulos and G. R. Dvali, Phys. Lett. B429, 263 (1998), [hep$\mathrm{ph} / 9803315]$

[382] I. Antoniadis, N. Arkani-Hamed, S. Dimopoulos and G. R. Dvali, Phys. Lett. B436, 257 (1998), [hep-ph/9804398].

[383] G. R. Dvali, G. Gabadadze and M. Porrati, Phys. Lett. B485, 208 (2000), [hep-th/0005016].

[384] L. Randall and R. Sundrum, Phys. Rev. Lett. 83, 4690 (1999), [hep-th/9906064].

[385] L. Randall and R. Sundrum, Phys. Rev. Lett. 83, 3370 (1999), [hep-ph/9905221].

[386] P. C. Argyres, S. Dimopoulos and J. March-Russell, Phys. Lett. B441, 96 (1998), [hepth/9808138].

[387] A. Kaus and H. S. Reall, 0901.4236.

[388] C. Clarkson and S. S. Seahra, Class. Quant. Grav. 22, 3653 (2005), [gr-qc/0505145].

[389] P. Kanti and R. A. Konoplya, Phys. Rev. D73, 044002 (2006), [hep-th/0512257].

[390] P. Kanti, R. A. Konoplya and A. Zhidenko, Phys. Rev. D74, 064008 (2006), [gr-qc/0607048].

[391] U. A. al Binni and G. Siopsis, Phys. Rev. D76, 104031 (2007), [0708.3363].

[392] S. Chen, B. Wang and R.-K. Su, Phys. Lett. B647, 282 (2007), [hep-th/0701209].

[393] M. Nozawa and T. Kobayashi, Phys. Rev. D78, 064006 (2008), [0803.3317].

[394] P. T. Leung, Y. T. Liu, W. M. Suen, C. Y. Tam and K. Young, Phys. Rev. Lett. 78, 2894 (1997), [gr-qc/9903031].

[395] P. T. Leung, Y. T. Liu, W. M. Suen, C. Y. Tam and K. Young, Phys. Rev. D59, 044034 (1999), [gr-qc/9903032].

[396] S. M. Carroll, Phys. Rev. Lett. 81, 3067 (1998), [astro-ph/9806099].

[397] I. Zlatev, L.-M. Wang and P. J. Steinhardt, Phys. Rev. Lett. 82, 896 (1999), [astro$\mathrm{ph} / 9807002]$

[398] R. R. Caldwell, Phys. Lett. B545, 23 (2002), [astro-ph/9908168].

[399] B. McInnes, JHEP 08, 029 (2002), [hep-th/0112066].

[400] S.-b. Chen and J.-l. Jing, Class. Quant. Grav. 22, 4651 (2005), [gr-qc/0511085].

[401] Y. Zhang, Y. X. Gui and F. Li, Gen. Rel. Grav. 39, 1003 (2007), [gr-qc/0612010]. 
[402] C. Ma, Y. Gui, W. Wang and F. Wang, Central Eur. J. Phys. 6, 194 (2008), [gr-qc/0611146].

[403] Y. Zhang and Y. X. Gui, Class. Quant. Grav. 23, 6141 (2006), [gr-qc/0612009].

[404] Y. Zhang, Y.-X. Gui and F. Yu, Chin. Phys. Lett. 26, 030401 (2009), [0710.5064].

[405] N. Varghese and V. C. Kuriakose, 0802.1397.

[406] S.-b. Chen and J.-l. Jing, JHEP 03, 081 (2009), [0812.2600].

[407] J. Polchinski, Phys. Rev. Lett. 75, 4724 (1995), [hep-th/9510017].

[408] G. 't Hooft, gr-qc/9310026.

[409] L. Susskind, J. Math. Phys. 36, 6377 (1995), [hep-th/9409089].

[410] D. T. Son and A. O. Starinets, JHEP 03, 052 (2006), [hep-th/0601157].

[411] D. Marolf, JHEP 05, 042 (2005), [hep-th/0412032].

[412] D. Birmingham, Phys. Rev. D64, 064024 (2001), [hep-th/0101194].

[413] G. Policastro, D. T. Son and A. O. Starinets, JHEP 12, 054 (2002), [hep-th/0210220].

[414] I. Amado, M. Kaminski and K. Landsteiner, 0903.2209.

[415] G. Policastro, D. T. Son and A. O. Starinets, JHEP 09, 043 (2002), [hep-th/0205052].

[416] R. Baier, P. Romatschke, D. T. Son, A. O. Starinets and M. A. Stephanov, JHEP 04, 100 (2008), [0712.2451].

[417] G. Policastro, JHEP 02, 034 (2009), [0812.0992].

[418] S. A. Hartnoll and S. Prem Kumar, JHEP 12, 036 (2005), [hep-th/0508092].

[419] A. Buchel, J. T. Liu and A. O. Starinets, Nucl. Phys. B707, 56 (2005), [hep-th/0406264].

[420] A. Buchel, Nucl. Phys. B803, 166 (2008), [0805.2683].

[421] R. C. Myers, M. F. Paulos and A. Sinha, Phys. Rev. D79, 041901 (2009), [0806.2156].

[422] M. Haack and A. Yarom, Nucl. Phys. B813, 140 (2009), [0811.1794].

[423] A. Ritz and J. Ward, 0811.4195.

[424] R. C. Myers, M. F. Paulos and A. Sinha, 0903.2834.

[425] S. Cremonini, K. Hanaki, J. T. Liu and P. Szepietowski, 0903.3244.

[426] A. Buchel and J. T. Liu, Phys. Rev. Lett. 93, 090602 (2004), [hep-th/0311175].

[427] P. Kovtun, D. T. Son and A. O. Starinets, Phys. Rev. Lett. 94, 111601 (2005), [hepth/0405231].

[428] A. Buchel, Phys. Lett. B609, 392 (2005), [hep-th/0408095].

[429] K. Maeda, M. Natsuume and T. Okamura, Phys. Rev. D73, 066013 (2006), [hep-th/0602010].

[430] J. Mas, J. P. Shock and J. Tarrio, JHEP 01, 025 (2009), [0811.1750].

[431] J. Mas, J. P. Shock and J. Tarrio, 0812.4432.

[432] X.-H. Ge, Y. Matsuo, F.-W. Shu, S.-J. Sin and T. Tsukioka, Prog. Theor. Phys. 120, 833 (2008), [0806.4460].

[433] J. Erdmenger, M. Haack, M. Kaminski and A. Yarom, JHEP 01, 055 (2009), [0809.2488].

[434] S. Cremonini, K. Hanaki, J. T. Liu and P. Szepietowski, 0812.3572.

[435] Y. Matsuo, S.-J. Sin, S. Takeuchi, T. Tsukioka and C.-M. Yoo, 0901.0610.

[436] P. Kovtun, D. T. Son and A. O. Starinets, JHEP 10, 064 (2003), [hep-th/0309213].

[437] M. Brigante, H. Liu, R. C. Myers, S. Shenker and S. Yaida, Phys. Rev. D77, 126006 (2008), [0712.0805].

[438] Y. Kats and P. Petrov, JHEP 01, 044 (2009), [0712.0743].

[439] M. Brigante, H. Liu, R. C. Myers, S. Shenker and S. Yaida, Phys. Rev. Lett. 100, 191601 (2008), [0802.3318].

[440] A. Buchel, R. C. Myers and A. Sinha, JHEP 03, 084 (2009), [0812.2521].

[441] A. Buchel, R. C. Myers, M. F. Paulos and A. Sinha, Phys. Lett. B669, 364 (2008), [0808.1837].

[442] R.-G. Cai, Z.-Y. Nie, N. Ohta and Y.-W. Sun, Phys. Rev. D79, 066004 (2009), [0901.1421].

[443] N. Banerjee and S. Dutta, JHEP 03, 116 (2009), [0901.3848].

[444] X.-H. Ge and S.-J. Sin, 0903.2527.

[445] N. Banerjee and S. Dutta, 0903.3925.

[446] J. Mas and J. Tarrio, JHEP 05, 036 (2007), [hep-th/0703093].

[447] K. Maeda, M. Natsuume and T. Okamura, Phys. Rev. D72, 086012 (2005), [hep-th/0509079].

[448] I. Kanitscheider and K. Skenderis, JHEP 04, 062 (2009), [0901.1487].

[449] R. A. Janik and R. B. Peschanski, Phys. Rev. D74, 046007 (2006), [hep-th/0606149].

[450] C. Hoyos-Badajoz, K. Landsteiner and S. Montero, JHEP 04, 031 (2007), [hep-th/0612169].

[451] R. C. Myers, A. O. Starinets and R. M. Thomson, JHEP 11, 091 (2007), [0706.0162].

[452] J. Erdmenger, M. Kaminski and F. Rust, Phys. Rev. D77, 046005 (2008), [0710.0334].

[453] R. C. Myers and A. Sinha, JHEP 06, 052 (2008), [0804.2168].

[454] N. Evans and E. Threlfall, Phys. Rev. D78, 105020 (2008), [0805.0956].

[455] N. Evans and E. Threlfall, Phys. Rev. D77, 126008 (2008), [0802.0775].

[456] A. Paredes, K. Peeters and M. Zamaklar, JHEP 05, 027 (2008), [0803.0759].

[457] M. Ammon, J. Erdmenger, M. Kaminski and P. Kerner, 0903.1864. 
[458] C. V. Johnson and A. Kundu, 0904.4320.

[459] G. W. Gibbons, Commun. Math. Phys. 44, 245 (1975).

[460] R. D. Blandford and R. L. Znajek, Mon. Not. Roy. Astron. Soc. 179, 433 (1977).

[461] O. Dreyer et al., Class. Quant. Grav. 21, 787 (2004), [gr-qc/0309007].

[462] E. Berti, V. Cardoso and C. M. Will, AIP Conf. Proc. 873, 82 (2006).

[463] E. Berti and V. Cardoso, Int. J. Mod. Phys. D15, 2209 (2006), [gr-qc/0605101].

[464] R. A. Remillard and J. E. McClintock, Ann. Rev. Astron. Astrophys. 44, 49 (2006), [astro$\mathrm{ph} / 0606352]$.

[465] F. Melia, 0705.1537.

[466] M. C. Miller and E. J. M. Colbert, Int. J. Mod. Phys. D13, 1 (2004), [astro-ph/0308402].

[467] J. R. Hurley, Mon. Not. Roy. Astron. Soc. 379, 93 (2007), [0705.0748].

[468] C. T. Berghea, K. A. Weaver, E. J. M. Colbert and T. P. Roberts, 0807.1547.

[469] K. Thorne, 300 Years of Gravitation S.W. Hawking and W. Israel (Editors) (Cambridge University Press, Cambridge, 1987).

[470] LIGO Scientific, L. M. Goggin, Class. Quant. Grav. 23, S709 (2006).

[471] The LIGO Scientific, . B. Abbott, 0905.1654.

[472] H. Nakano, H. Takahashi, H. Tagoshi and M. Sasaki, Phys. Rev. D68, 102003 (2003), [grqc/0306082].

[473] H. Nakano, H. Takahashi, H. Tagoshi and M. Sasaki, Prog. Theor. Phys. 111, 781 (2004), [gr-qc/0403069].

[474] Y. Tsunesada et al., Phys. Rev. D71, 103005 (2005), [gr-qc/0410037].

[475] E. Berti, V. Cardoso, J. A. Gonzalez, U. Sperhake and B. Bruegmann, Class. Quant. Grav. 25, 114035 (2008), [0711.1097].

[476] E. N. Dorband, E. Berti, P. Diener, E. Schnetter and M. Tiglio, Phys. Rev. D74, 084028 (2006), [gr-qc/0608091].

[477] A. Buonanno, G. B. Cook and F. Pretorius, Phys. Rev. D75, 124018 (2007), [gr-qc/0610122].

[478] L. Bildsten and C. Cutler, Astrophys. J. 400, 175 (1992).

[479] L. Gualtieri, E. Berti, J. A. Pons, G. Miniutti and V. Ferrari, Phys. Rev. D64, 104007 (2001), [gr-qc/0107046].

[480] J. A. Pons, E. Berti, L. Gualtieri, G. Miniutti and V. Ferrari, Phys. Rev. D65, 104021 (2002), [gr-qc/0111104].

[481] E. E. Flanagan and T. Hinderer, Phys. Rev. D77, 021502 (2008), [0709.1915].

[482] E. Berti and M. Volonteri, Astrophys. J. 684, 822 (2008), [0802.0025].

[483] C. O. Lousto and R. H. Price, Phys. Rev. D55, 2124 (1997), [gr-qc/9609012].

[484] A. Nagar, O. Zanotti, J. A. Font and L. Rezzolla, Phys. Rev. D75, 044016 (2007), [grqc/0610131].

[485] M. Shibata, K. Kyutoku, T. Yamamoto and K. Taniguchi, Phys. Rev. D79, 044030 (2009), [0902.0416].

[486] R. A. Araya-Gochez, Mon. Not. Roy. Astron. Soc. 355, 336 (2003), [astro-ph/0311001].

[487] C. L. Fryer and K. C. B. New, Living Reviews in Relativity 6 (2003).

[488] K. Kotake, K. Sato and K. Takahashi, Rept. Prog. Phys. 69, 971 (2006), [astro-ph/0509456].

[489] H.-T. Janka, K. Langanke, A. Marek, G. Martinez-Pinedo and B. Mueller, Phys. Rept. 442, 38 (2007), [astro-ph/0612072].

[490] C. D. Ott, Class. Quant. Grav. 26, 063001 (2009), [0809.0695].

[491] E. Seidel and T. Moore, Phys. Rev. D35, 2287 (1987).

[492] E. Seidel, E. S. Myra and T. Moore, Phys. Rev. D38, 2349 (1988).

[493] E. Seidel, Phys. Rev. D42, 1884 (1990).

[494] E. Seidel, Phys. Rev. D44, 950 (1991).

[495] T. Harada, H. Iguchi and M. Shibata, Phys. Rev. D68, 024002 (2003), [gr-qc/0305058].

[496] H. Sotani, S. Yoshida and K. D. Kokkotas, Phys. Rev. D75, 084015 (2007), [gr-qc/0702091].

[497] H. Sotani, 0904.1465.

[498] T. Nakamura, Prog. Theor. Phys. 65, 1876 (1981).

[499] L. Baiotti et al., Phys. Rev. D71, 024035 (2005), [gr-qc/0403029].

[500] L. Baiotti, I. Hawke, L. Rezzolla and E. Schnetter, Phys. Rev. Lett. 94, 131101 (2005), [grqc/0503016].

[501] L. Baiotti, I. Hawke and L. Rezzolla, Class. Quant. Grav. 24, S187 (2007), [gr-qc/0701043].

[502] E. Mueller, M. Rampp, R. Buras, H. T. Janka and D. H. Shoemaker, Astrophys. J. 603, 221 (2004), [astro-ph/0309833].

[503] M. Shibata, Phys. Rev. D60, 104052 (1999), [gr-qc/9908027].

[504] M. Shibata and K. Uryu, Prog. Theor. Phys. 107, 265 (2002), [gr-qc/0203037].

[505] M. Shibata, K. Taniguchi and K. Uryu, Phys. Rev. D68, 084020 (2003), [gr-qc/0310030]. 
[506] M. Shibata, K. Taniguchi and K. Uryu, Phys. Rev. D71, 084021 (2005), [gr-qc/0503119].

[507] M. Shibata and K. Taniguchi, Phys. Rev. D73, 064027 (2006), [astro-ph/0603145].

[508] T. Yamamoto, M. Shibata and K. Taniguchi, 0806.4007.

[509] M. Anderson et al., Phys. Rev. Lett. 100, 191101 (2008), [0801.4387].

[510] Y. T. Liu, S. L. Shapiro, Z. B. Etienne and K. Taniguchi, Phys. Rev. D78, 024012 (2008), [0803.4193].

[511] L. Baiotti, B. Giacomazzo and L. Rezzolla, 0901.4955.

[512] K. Belczynski, R. E. Taam, E. Rantsiou and M. van der Sluys, astro-ph/0703131.

[513] J. M. Silverman and A. V. Filippenko, 0802.2716.

[514] M. Shibata and K. Uryu, Class. Quant. Grav. 24, S125 (2007), [astro-ph/0611522].

[515] M. Shibata and K. Uryu, Phys. Rev. D74, 121503 (2006), [gr-qc/0612142].

[516] M. Shibata and K. Taniguchi, Phys. Rev. D77, 084015 (2008), [0711.1410].

[517] Z. B. Etienne et al., Phys. Rev. D77, 084002 (2008), [0712.2460].

[518] Z. B. Etienne, Y. T. Liu, S. L. Shapiro and T. W. Baumgarte, 0812.2245.

[519] M. D. Duez et al., Phys. Rev. D78, 104015 (2008), [0809.0002].

[520] C. Cutler and E. E. Flanagan, Phys. Rev. D49, 2658 (1994), [gr-qc/9402014].

[521] N. Seto, Phys. Rev. D75, 024016 (2007), [astro-ph/0512212].

[522] J. Kanner et al., Class. Quant. Grav. 25, 184034 (2008), [0803.0312].

[523] J. S. Bloom et al., 0902.1527.

[524] S. Nissanke, S. A. Hughes, D. E. Holz, N. Dalal and J. L. Sievers, 0904.1017.

[525] B. Aylott et al., 0901.4399.

[526] M. Hannam, 0901.2931.

[527] A. Buonanno et al., Phys. Rev. D76, 104049 (2007), [0706.3732].

[528] J. G. Baker et al., Phys. Rev. D78, 044046 (2008), [0805.1428].

[529] E. Berti, V. Cardoso, J. A. Gonzalez and U. Sperhake, Phys. Rev. D75, 124017 (2007), [gr-qc/0701086].

[530] U. Sperhake et al., Phys. Rev. D78, 064069 (2008), [0710.3823].

[531] I. Hinder, B. Vaishnav, F. Herrmann, D. Shoemaker and P. Laguna, Phys. Rev. D77, 081502 (2008), [0710.5167].

[532] B. Vaishnav, I. Hinder, F. Herrmann and D. Shoemaker, Phys. Rev. D76, 084020 (2007), [0705.3829].

[533] D. Shoemaker, B. Vaishnav, I. Hinder and F. Herrmann, Class. Quant. Grav. 25, 114047 (2008), [0802.4427].

[534] M. Luna and A. M. Sintes, Class. Quant. Grav. 23, 3763 (2006), [gr-qc/0601072].

[535] P. Ajith and S. Bose, 0901.4936.

[536] A. Buonanno, L. E. Kidder and L. Lehner, Phys. Rev. D77, 026004 (2008), [0709.3839].

[537] L. Rezzolla et al., Phys. Rev. D78, 044002 (2008), [0712.3541].

[538] L. Rezzolla, Class. Quant. Grav. 26, 094023 (2009), [0812.2325].

[539] E. Barausse and L. Rezzolla, 0904.2577.

[540] T. Bogdanovic, C. S. Reynolds and M. C. Miller, astro-ph/0703054.

[541] M. Kesden et al., in preparation.

[542] J. Rhoades, Clifford E. and R. Ruffini, Phys. Rev. Lett. 32, 324 (1974).

[543] T. Bulik, K. Belczynski and A. Prestwich, 0803.3516.

[544] I. Mandel, D. A. Brown, J. R. Gair and M. C. Miller, Astrophys. J. 681, 1431 (2007), [0705.0285].

[545] J. M. Fregeau, S. L. Larson, M. C. Miller, R. W. O'Shaughnessy and F. A. Rasio, Astrophys. J. 646, L135 (2006), [astro-ph/0605732].

[546] J. Kormendy and D. Richstone, Ann. Rev. Astron. Astrophys. 33, 581 (1995).

[547] E. Magorrian et al., Astron. J. 115, 2285 (1998).

[548] R. J. McLure and J. S. Dunlop, Mon. Not. Roy. Astron. Soc. 331, 795 (2002).

[549] R. Schodel et al., Nature 419, 694 (2002).

[550] A. M. Ghez et al., 0808.2870.

[551] Z.-Q. Shen, K. Y. Lo, M. C. Liang, P. T. P. Ho and J. H. Zhao, Nature 438, 62 (2005), [astro-ph/0512515].

[552] E. Maoz, Astrophys. J. 494, L181 (1998), [astro-ph/9710309].

[553] M. Coleman Miller, Mon. Not. Roy. Astron. Soc. Lett. 367, L32 (2006), [astro-ph/0512194].

[554] V. Cardoso, P. Pani, M. Cadoni and M. Cavaglia, Class. Quant. Grav. 25, 195010 (2008), [0808.1615].

[555] P. Pani, V. Cardoso, M. Cadoni and M. Cavaglia, 0901.0850.

[556] J. R. Herrnstein et al., Nature 400, 539 (1999).

[557] K. Gebhardt et al., Astrophys. J. 543, L5 (2000), [astro-ph/0007123]. 
[558] A. W. Graham, 0807.2549.

[559] D. Merritt and L. Ferrarese, Mon. Not. Roy. Astron. Soc. 320, L30 (2001), [astro-ph/0009076].

[560] L. Ferrarese and D. Merritt, Astrophys. J. 539, L9 (2000), [astro-ph/0006053].

[561] A. Marconi and L. K. Hunt, Astrophys. J. 589, L21 (2003), [astro-ph/0304274].

[562] D. Merritt and M. Milosavljevic, Living Rev. Rel. 8, 8 (2005), [astro-ph/0410364].

[563] P. F. Hopkins et al., Astrophys. J. Suppl. 163, 1 (2006), [astro-ph/0506398].

[564] P. F. Hopkins et al., Astrophys. J. 630, 705 (2005), [astro-ph/0504190].

[565] M. C. Begelman, R. D. Blandford and M. J. Rees, Nature 287, 307 (1980).

[566] P. C. Peters and J. Mathews, Phys. Rev. 131, 435 (1963).

[567] E. Berti, Class. Quant. Grav. 23, S785 (2006), [astro-ph/0602470].

[568] M. Volonteri, F. Haardt and P. Madau, Astrophys. J. 582, 559 (2003), [astro-ph/0207276].

[569] M. Volonteri, P. Madau, E. Quataert and M. J. Rees, Astrophys. J. 620, 69 (2005), [astro$\mathrm{ph} / 0410342]$.

[570] A. Sesana, M. Volonteri and F. Haardt, Mon. Not. Roy. Astron. Soc. 377, 1711 (2007), [astro$\mathrm{ph} / 0701556]$.

[571] S. M. Koushiappas and A. R. Zentner, Astrophys. J. 639, 7 (2006), [astro-ph/0503511].

[572] L. A. Gergely and B. Mikoczi, 0808.1704.

[573] L. A. Gergely and P. L. Biermann, 0704.1968.

[574] G. Taffoni, L. Mayer, M. Colpi and F. Governato, Mon. Not. Roy. Astron. Soc. 341, 434 (2003), [astro-ph/0301271].

[575] S. Komossa, AIP Conf. Proc. 686, 161 (2003), [astro-ph/0306439].

[576] S. Komossa et al., Astrophys. J. 582, L15 (2003), [astro-ph/0212099].

[577] L. Ballo et al., Astrophys. J. 600, 634 (2004), [astro-ph/0306436].

[578] M. Guainazzi, E. Piconcelli, E. Jimenez-Bailon and G. Matt, Astron. Astrophys. 429, L9 (2005), [astro-ph/0411435].

[579] D. A. Evans et al., 0712.2669.

[580] S. Bianchi, M. Chiaberge, E. Piconcelli, M. Guainazzi and G. Matt, 0802.0825.

[581] C. Rodriguez et al., Astrophys. J. 646, 49 (2006), [astro-ph/0604042].

[582] C. Rodriguez, G. B. Taylor, R. T. Zavala, Y. M. Pihlstrom and A. B. Peck, 0902.4444.

[583] M. J. Valtonen et al., Nature 452, 851 (2008), [0809.1280].

[584] T. A. Boroson and T. R. Lauer, 0901.3779.

[585] M. Volonteri, J. M. Miller and M. Dotti, 0903.3947.

[586] L. Brenneman et al., 0902.4691.

[587] J. E. McClintock and R. A. Remillard, 0902.3488.

[588] J. M. Miller et al., 0902.4677.

[589] A. R. King and U. Kolb, Mon. Not. Roy. Astron. Soc. 305, 654 (1999), [astro-ph/9901296].

[590] V. Kalogera, Astrophys. J. 541, 319 (2000), [astro-ph/9911417].

[591] R. O'Shaughnessy, J. Kaplan, V. Kalogera and K. Belczynski, Astrophys. J. 632, 1035 (2005), [astro-ph/0503219].

[592] P. Grandclement, M. Ihm, V. Kalogera and K. Belczynski, Phys. Rev. D69, 102002 (2004), [gr-qc/0312084].

[593] C. F. Gammie, S. L. Shapiro and J. C. McKinney, Astrophys. J. 602, 312 (2004), [astro$\mathrm{ph} / 0310886]$.

[594] P. Madau and M. J. Rees, Astrophys. J. 551, L27 (2001), [astro-ph/0101223].

[595] T. Ohkubo et al., Astrophys. J. 645, 1352 (2006), [astro-ph/0507593].

[596] Y. Suwa, T. Takiwaki, K. Kotake and K. Sato, Publ. Astron. Soc. Jap. 59, 771 (2007), [0704.1945].

[597] M. Shibata and S. L. Shapiro, Astrophys. J. 572, L39 (2002), [astro-ph/0205091].

[598] S. L. Shapiro and M. Shibata, Astrophys. J. 577, 904 (2002), [astro-ph/0209251].

[599] S. M. Koushiappas, J. S. Bullock and A. Dekel, Mon. Not. Roy. Astron. Soc. 354, 292 (2004), [astro-ph/0311487].

[600] M. C. Begelman, M. Volonteri and M. J. Rees, Mon. Not. Roy. Astron. Soc. 370, 289 (2006), [astro-ph/0602363].

[601] C. d. P. Lagos, N. D. Padilla and S. A. Cora, 0901.0547.

[602] S. A. Hughes and R. D. Blandford, Astrophys. J. 585, L101 (2003), [astro-ph/0208484].

[603] A. R. King, S. H. Lubow, G. I. Ogilvie and J. E. Pringle, Mon. Not. Roy. Astron. Soc. 363, 49 (2005), [astro-ph/0507098].

[604] A. R. King and J. E. Pringle, Mon. Not. Roy. Astron. Soc. Lett. 373, L93 (2006), [astro$\mathrm{ph} / 0609598]$.

[605] A. R. King, J. E. Pringle and J. A. Hofmann, 0801.1564.

[606] J. M. Wang et al., 0904.1896. 
[607] L. W. Brenneman and C. S. Reynolds, Astrophys. J. 652, 1028 (2006), [astro-ph/0608502].

[608] J.-X. Wang and D. Merritt, Astrophys. J. 600, 149 (2004), [astro-ph/0305493].

[609] A. Soltan, Mon. Not. Roy. Astron. Soc. 200, 114 (1982).

[610] R. A. Daly, Astrophys. J. 696, L32 (2009), [0903.4861].

[611] B. R. McNamara et al., 0811.3020.

[612] J. M. Miller, C. S. Reynolds, A. C. Fabian, G. Miniutti and L. C. Gallo, 0902.2840.

[613] S. W. Davis, C. Done and O. M. Blaes, Astrophys. J. 647, 525 (2006), [astro-ph/0602245].

[614] R. Shafee et al., Astrophys. J. 636, L113 (2006), [astro-ph/0508302].

[615] R. C. Reis, A. C. Fabian, R. R. Ross and J. M. Miller, 0902.1745.

[616] J. Liu, J. McClintock, R. Narayan, S. Davis and J. Orosz, Astrophys. J. 679, L37 (2008), [0803.1834].

[617] M. Middleton, C. Done, M. Gierlinski and S. Davis, Mon. Not. Roy. Astron. Soc. 373, 1004 (2006), [astro-ph/0601540].

[618] S. N. Zhang, W. Cui and W. Chen, Astrophys. J. 482, L155 (1997), [astro-ph/9704072].

[619] J. E. McClintock et al., Astrophys. J. 652, 518 (2006), [astro-ph/0606076].

[620] L. Gou et al., 0901.0920.

[621] J. M. Miller et al., Astrophys. J. 679, L113 (2008), [0802.3882].

[622] T. E. Strohmayer, Astrophys. J. 552, L49 (2001), [astro-ph/0104487].

[623] J. M. Miller, A. C. Fabian, M. A. Nowak and W. H. G. Lewin, astro-ph/0402101.

[624] G. Miniutti et al., 0905.2891.

[625] A. C. Fabian, G. Miniutti, K. Iwasawa and R. R. Ross, Mon. Not. Roy. Astron. Soc. 361, 795 (2005), [astro-ph/0504472].

[626] M. Elvis, G. Risaliti and G. Zamorani, Astrophys. J. 565, L75 (2002), [astro-ph/0112413].

[627] Q.-j. Yu and S. Tremaine, Mon. Not. Roy. Astron. Soc. 335, 965 (2002), [astro-ph/0203082].

[628] J.-M. Wang, Y.-M. Chen, L. C. Ho and R. J. McLure, Astrophys. J. 642, L111 (2006), [astro$\mathrm{ph} / 0603813]$.

[629] J.-M. Wang, Y.-R. Li, J.-C. Li and S. Zhang, 0802.4322.

[630] X. Cao and F. Li, 0808.0759.

[631] F. Shankar, D. H. Weinberg and J. Miralda-Escude, Astrophys. J. 690, 20 (2009), [0710.4488].

[632] A. Merloni and S. Heinz, 0805.2499.

[633] R. A. Daly, Astrophys. J. 691, L72 (2009), [0811.1897].

[634] P. Ajith et al., Phys. Rev. D77, 104017 (2008), [0710.2335].

[635] A. Sesana, J. Gair, I. Mandel and A. Vecchio, 0903.4177.

[636] R. K. Kopparapu et al., Astrophys. J. 675, 1459 (2008), [0706.1283].

[637] E. S. Phinney, Astrophys. J. 380, L17 (1991).

[638] V. Kalogera et al., Astrophys. J. 601, L179 (2004), [astro-ph/0312101].

[639] V. Kalogera et al., Astrophys. J. 614, L137 (2004).

[640] R. O'Shaughnessy, C. Kim, T. Frakgos, V. Kalogera and K. Belczynski, Astrophys. J. 633, 1076 (2005), [astro-ph/0504479].

[641] R. O'Shaughnessy, C. Kim, V. Kalogera and K. Belczynski, Astrophys. J. 672, 479 (2008), [astro-ph/0610076].

[642] D. A. Brown et al., Phys. Rev. Lett. 99, 201102 (2007), [gr-qc/0612060].

[643] I. Mandel and J. R. Gair, 0811.0138.

[644] K. Belczynski, V. Kalogera, F. A. Rasio, R. E. Taam and T. Bulik, Astrophys. J. 662, 504 (2007), [astro-ph/0612032].

[645] M. C. Miller and V. M. Lauburg, Astrophys. J. 692, 917 (2009), [0804.2783].

[646] R. M. O'Leary, F. A. Rasio, J. M. Fregeau, N. Ivanova and R. O'Shaughnessy, Astrophys. J. 637, 937 (2006), [astro-ph/0508224].

[647] R. O'Leary, R. O'Shaughnessy and F. Rasio, Phys. Rev. D76, 061504 (2007), [astro$\mathrm{ph} / 0701887]$.

[648] R. M. O'Leary, B. Kocsis and A. Loeb, 0807.2638.

[649] M. G. Haehnelt, Class. Quant. Grav. 20, S31 (2003).

[650] M. Enoki, K. T. Inoue, M. Nagashima and N. Sugiyama, Astrophys. J. 615, 19 (2004), [astro$\mathrm{ph} / 0404389]$.

[651] K. Menou, Z. Haiman and V. K. Narayanan, Astrophys. J. 558, 535 (2001), [astro-ph/0101196].

[652] K. J. Rhook and J. S. B. Wyithe, Mon. Not. Roy. Astron. Soc. 361, 1145 (2005), [astro$\mathrm{ph} / 0503210]$.

[653] J. S. B. Wyithe and A. Loeb, Astrophys. J. 590, 691 (2003), [astro-ph/0211556].

[654] K. G. Arun et al., 0811.1011.

[655] R. R. Islam, J. E. Taylor and J. Silk, Mon. Not. Roy. Astron. Soc. 354, 629 (2004), [astro$\mathrm{ph} / 0309559]$ 
[656] T. Tanaka and Z. Haiman, Astrophys. J. 696, 1798 (2009), [0807.4702].

[657] M. C. Miller, Astrophys. J. 581, 438 (2002), [astro-ph/0206404].

[658] C. M. Will, Astrophys. J. 611, 1080 (2004), [astro-ph/0403644].

[659] T. Matsubayashi, H. Shinkai and T. Ebisuzaki, Astrophys. J. 614, 864 (2004).

[660] M. C. Miller, Astrophys. J. 618, 426 (2004), [astro-ph/0409331].

[661] S. F. Portegies Zwart et al., astro-ph/0511397.

[662] T. A. Apostolatos, Phys. Rev. D52, 605 (1995).

[663] B. J. Owen, Phys. Rev. D53, 6749 (1996), [gr-qc/9511032].

[664] C. B. M. H. Chirenti and L. Rezzolla, Class. Quant. Grav. 24, 4191 (2007), [0706.1513].

[665] F. D. Ryan, Phys. Rev. D52, 5707 (1995).

[666] F. D. Ryan, Phys. Rev. D56, 1845 (1997).

[667] C. Li and G. Lovelace, Phys. Rev. D77, 064022 (2008), [gr-qc/0702146].

[668] L. Blanchet, Living Rev. Rel. 5, 3 (2002), [gr-qc/0202016].

[669] P. Ajith et al., Class. Quant. Grav. 24, S689 (2007), [0704.3764].

[670] Y. Pan et al., Phys. Rev. D77, 024014 (2008), [0704.1964].

[671] M. Boyle, D. A. Brown and L. Pekowsky, 0901.1628.

[672] S. Dain, C. O. Lousto and Y. Zlochower, Phys. Rev. D78, 024039 (2008), [0803.0351].

[673] A. Buonanno and T. Damour, Phys. Rev. D62, 064015 (2000), [gr-qc/0001013].

[674] T. Damour and A. Gopakumar, Phys. Rev. D73, 124006 (2006), [gr-qc/0602117].

[675] L. Blanchet, M. S. S. Qusailah and C. M. Will, Astrophys. J. 635, 508 (2005), [astro$\mathrm{ph} / 0507692]$.

[676] J. D. Schnittman et al., Phys. Rev. D77, 044031 (2008), [0707.0301].

[677] J. D. Schnittman and A. Buonanno, Astrophys. J. 662, L63 (2007), [astro-ph/0702641].

[678] S. Babak, M. Hannam, S. Husa and B. F. Schutz, 0806.1591.

[679] J. I. Thorpe et al., 0811.0833.

[680] M. Hannam et al., 0901.2437.

[681] S. A. Hughes and K. Menou, Astrophys. J. 623, 689 (2005), [astro-ph/0410148].

[682] A. Corichi, Phys. Rev. D67, 087502 (2003), [gr-qc/0212126].

[683] J. Oppenheim, Phys. Rev. D69, 044012 (2004), [gr-qc/0307089].

[684] Y. Ling and H.-b. Zhang, Phys. Rev. D68, 101501 (2003), [gr-qc/0309018].

[685] M. R. Setare, Phys. Rev. D69, 044016 (2004), [hep-th/0312061].

[686] C. Kiefer, Class. Quant. Grav. 21, L123 (2004), [gr-qc/0406097].

[687] M. R. Setare and E. C. Vagenas, Mod. Phys. Lett. A20, 1923 (2005), [hep-th/0401187].

[688] M. Maggiore, Phys. Rev. Lett. 100, 141301 (2008), [0711.3145].

[689] E. C. Vagenas, JHEP 11, 073 (2008), [0804.3264].

[690] A. J. M. Medved, Class. Quant. Grav. 25, 205014 (2008), [0804.4346].

[691] S.-W. Wei, R. Li, Y.-X. Liu and J.-R. Ren, 0901.0587.

[692] J. D. Bekenstein, Lett. Nuovo Cim. 11, 467 (1974).

[693] J. D. Bekenstein and V. F. Mukhanov, Phys. Lett. B360, 7 (1995), [gr-qc/9505012].

[694] I. B. Khriplovich, Sov. Phys. JETP, 99:460 (2004), [gr-qc/0404083].

[695] J. D. Bekenstein, Phys. Rev. D7, 2333 (1973).

[696] J. D. Bekenstein, gr-qc/9808028.

[697] S. S. Gubser and I. Mitra, hep-th/0009126.

[698] S. S. Gubser and I. Mitra, JHEP 08, 018 (2001), [hep-th/0011127].

[699] H. S. Reall, Phys. Rev. D64, 044005 (2001), [hep-th/0104071].

[700] T. Harmark, V. Niarchos and N. A. Obers, Class. Quant. Grav. 24, R1 (2007), [hepth/0701022].

[701] G. Koutsoumbas, S. Musiri, E. Papantonopoulos and G. Siopsis, JHEP 10, 006 (2006), [hepth/0606096].

[702] J. Shen, B. Wang, C.-Y. Lin, R.-G. Cai and R.-K. Su, JHEP 07, 037 (2007), [hep-th/0703102].

[703] X.-P. Rao, B. Wang and G.-H. Yang, Phys. Lett. B649, 472 (2007), [0712.0645].

[704] Y. S. Myung, Phys. Lett. B663, 111 (2008), [0801.2434].

[705] G. Koutsoumbas, E. Papantonopoulos and G. Siopsis, JHEP 05, 107 (2008), [0801.4921].

[706] G. Koutsoumbas, E. Papantonopoulos and G. Siopsis, Class. Quant. Grav. 26, 105004 (2009), [0806.1452].

[707] P. C. W. Davies, Proc. Roy. Soc. Lond. A353, 499 (1977).

[708] P. C. W. Davies, Class. Quant. Grav. 6, 1909 (1989).

[709] E. Berti and V. Cardoso, Phys. Rev. D77, 087501 (2008), [0802.1889].

[710] S. Hod, Phys. Rev. D75, 064013 (2007), [gr-qc/0611004].

[711] S. Hod, Class. Quant. Grav. 24, 4235 (2007), [0705.2306].

[712] S. Hod, Phys. Lett. B666, 483 (2008), [0810.5419]. 
[713] S. Hod, 0905.4113.

[714] R. J. Gleiser, C. O. Nicasio, R. H. Price and J. Pullin, Phys. Rept. 325, 41 (2000), [grqc/9807077].

[715] A. Garat and R. H. Price, Phys. Rev. D61, 044006 (2000).

[716] K. Ioka and H. Nakano, Phys. Rev. D76, 061503 (2007), [0704.3467].

[717] H. Nakano and K. Ioka, Phys. Rev. D76, 084007 (2007), [0708.0450].

[718] S. Okuzumi, K. Ioka and M. Sakagami, Phys. Rev. D77, 124018 (2008), [0803.0501].

[719] M. Favata, 0812.0069.

[720] D. Brizuela, J. M. Martin-Garcia and G. A. Mena Marugan, Phys. Rev. D74, 044039 (2006), [gr-qc/0607025].

[721] D. Brizuela, J. M. Martin-Garcia and G. A. M. Marugan, Phys. Rev. D76, 024004 (2007), [gr-qc/0703069].

[722] D. Brizuela, J. M. Martin-Garcia and G. A. M. Marugan, 0807.0824.

[723] B. Kol, 0709.3254.

[724] D. Brizuela, J. M. Martin-Garcia and M. Tiglio, 0903.1134.

[725] W. G. Unruh, Phys. Rev. Lett. 46, 1351 (1981).

[726] M. Visser, Class. Quant. Grav. 15, 1767 (1998), [gr-qc/9712010].

[727] M. Novello, M. Visser and G. Volovik, Artificial Black Holes (World Scientific, 2002).

[728] E. Berti, V. Cardoso and J. P. S. Lemos, Phys. Rev. D70, 124006 (2004), [gr-qc/0408099].

[729] S. Lepe and J. Saavedra, Phys. Lett. B617, 174 (2005), [gr-qc/0410074].

[730] S.-B. Chen and J.-L. Jing, Chin. Phys. Lett. 23, 21 (2006).

[731] P. Xi and X.-Z. Li, Int. J. Mod. Phys. D16, 1211 (2007), [0709.3714].

[732] J. Saavedra, Mod. Phys. Lett. A21, 1601 (2006), [gr-qc/0508040].

[733] S. R. Dolan, E. S. Oliveira and L. C. B. Crispino, Phys. Rev. D79, 064014 (2009), [0904.0010].

[734] V. Cardoso, physics/0503042.

[735] M. Cadoni and P. Pani, Class. Quant. Grav. 23, 2427 (2006), [physics/0510164].

[736] S. Okuzumi and M.-a. Sakagami, Phys. Rev. D76, 084027 (2007), [gr-qc/0703070].

[737] E. Abdalla, R. A. Konoplya and A. Zhidenko, Class. Quant. Grav. 24, 5901 (2007), [0706.2489].

[738] H. Nakano, Y. Kurita, K. Ogawa and C.-M. Yoo, Phys. Rev. D71, 084006 (2005), [grqc/0411041].

[739] C. Barcelo, A. Cano, L. J. Garay and G. Jannes, Phys. Rev. D75, 084024 (2007), [grqc/0701173].

[740] T. K. Das, N. Bilic and S. Dasgupta, JCAP 0706, 009 (2007), [astro-ph/0604477].

[741] T. K. Das, 0704.3618.

[742] M. Parikh and F. Wilczek, Phys. Rev. D58, 064011 (1998), [gr-qc/9712077].

[743] R. M. Wald, Journal of Mathematics and Physics 14, 1453 (1973).

[744] F. Cooper, A. Khare and U. Sukhatme, Phys. Rept. 251, 267 (1995), [hep-th/9405029].

[745] A. Maassen van den Brink, Phys. Rev. D62, 064009 (2000), [gr-qc/0001032].

[746] N. Andersson, Class. Quant. Grav. 11, L39 (1994).

[747] P. T. Leung, A. Maassen van den Brink, K. W. Mak and K. Young, Class. Quant. Grav. 20, L217 (2003), [gr-qc/0301018].

[748] R. J. Gleiser and G. Dotti, Class. Quant. Grav. 23, 5063 (2006), [gr-qc/0604021].

[749] V. Cardoso and M. Cavaglia, Phys. Rev. D74, 024027 (2006), [gr-qc/0604101].

[750] G. Dotti, R. Gleiser and J. Pullin, Phys. Lett. B644, 289 (2007), [gr-qc/0607052].

[751] G. Dotti, R. J. Gleiser, I. F. Ranea-Sandoval and H. Vucetich, Class. Quant. Grav. 25, 245012 (2008), [0805.4306].

[752] S. Komossa, H. Zhou and H. Lu, Astrophys. J. 678, L81 (2008), [0804.4585].

[753] J. A. Gonzalez, U. Sperhake, B. Bruegmann, M. Hannam and S. Husa, Phys. Rev. Lett. 98, 091101 (2007), [gr-qc/0610154].

[754] M. Campanelli, C. O. Lousto, Y. Zlochower and D. Merritt, Astrophys. J. 659, L5 (2007), [gr-qc/0701164].

[755] M. Campanelli, C. O. Lousto, Y. Zlochower and D. Merritt, Phys. Rev. Lett. 98, 231102 (2007), [gr-qc/0702133].

[756] T. Bogdanovic, M. Eracleous and S. Sigurdsson, 0809.3262.

[757] M. Dotti et al., 0809.3446.

[758] G. A. Shields, E. W. Bonning and S. Salviander, 0810.2563.

[759] T. M. Heckman, J. H. Krolik, S. M. Moran, J. Schnittman and S. Gezari, Astrophys. J. 695, 363 (2009), [0810.1244].

[760] C. J. Conselice, J. S. Gallagher and R. F. G. Wyse, Astron. J. 122, 2281 (2001), [astro$\mathrm{ph} / 0108019]$.

[761] C. M. Gaskell, 0903.4447. 
[762] J. M. Wrobel and A. Laor, Astrophys. J. 699, L22 (2009), [0905.3566].

[763] R. R. Chornock et al., The Astronomer's Telegram 1955, 1 (2009).

[764] R. Chornock et al., 0906.0849.

[765] T. R. Lauer and T. A. Boroson, 0906.0020.

[766] M. J. Valtonen et al., Astrophys. J. 659, 1074 (2007).

[767] W. G. Laarakkers and E. Poisson, gr-qc/9709033.

[768] E. Berti and N. Stergioulas, Mon. Not. Roy. Astron. Soc. 350, 1416 (2004), [gr-qc/0310061].

[769] C. S. Reynolds and A. C. Fabian, 0711.4158.

[770] K. Beckwith, J. Hawley and J. Krolik, 0801.2974.

[771] T. J. Turner and L. Miller, 0902.0651.

[772] L. Miller, T. J. Turner and J. N. Reeves, 0803.2680.

[773] D. A. Liedahl and D. F. Torres, Can. J. Phys. 83, 1149 (2005), [astro-ph/0510093].

[774] C. S. Reynolds and M. A. Nowak, Phys. Rept. 377, 389 (2003), [astro-ph/0212065].

[775] A. C. Fabian and G. Miniutti, astro-ph/0507409.

[776] J. M. Miller, Ann. Rev. Astron. Astrophys. 45, 441 (2007), [0705.0540].

[777] J. M. Miller, astro-ph/0609447.

[778] R. Narayan, J. E. McClintock and R. Shafee, AIP Conf. Proc. 968, 265 (2008), [0710.4073].

[779] R. Shafee, R. Narayan and J. E. McClintock, 0705.2244.

[780] J. E. McClintock, R. Narayan and R. Shafee, 0707.4492.

[781] S. W. Davis and I. Hubeny, Astrophys. J. Suppl. 164, 530 (2006), [astro-ph/0602499].

[782] G. E. Brown, C.-H. Lee, E. Moreno-Mendez and F. M. Walter, astro-ph/0612461.

[783] M. A. Abramowicz, W. Kluzniak and J.-P. Lasota, Astron. Astrophys. 396, L31 (2002), [astro$\mathrm{ph} / 0207270]$.

[784] D. N. Page and K. S. Thorne, Astrophys. J. 191, 499 (1974).

[785] Y. Tanaka et al., Nature 375, 659 (1995).

[786] K. Nandra, I. M. George, R. F. Mushotzky, T. J. Turner and T. Yaqoob, Astrophys. J. 477, 602 (1997), [astro-ph/9606169].

[787] K. Nandra, P. M. O’Neill, I. M. George, J. N. Reeves and T. J. Turner, Astron. Nachr. 88, 789 (2006), [astro-ph/0610585].

[788] K. Nandra, P. M. O'Neill, I. M. George and J. N. Reeves, 0708.1305.

[789] J. N. Reeves et al., Astron. Nachr. 88, 789 (2006), [astro-ph/0610436].

[790] R. C. Reis et al., Mon. Not. Roy. Astron. Soc. 387, 1489 (2008), [0804.0238].

[791] L. Rezzolla, S. Yoshida, T. J. Maccarone and O. Zanotti, Mon. Not. Roy. Astron. Soc. 344, L37 (2003), [astro-ph/0307487].

[792] R. V. Wagoner, A. S. Silbergleit and M. Ortega-Rodriguez, Astrophys. J. 559, L25 (2001), [astro-ph/0107168].

[793] M. A. Abramowicz and W. Kluzniak, Astron. Astrophys. 374, L19 (2001), [astro-ph/0105077].

[794] A. Rafiee and P. B. Hall, Astrophys. J. 691, 425 (2009), [0803.2335].

[795] D. Sijacki, V. Springel and M. G. Haehnelt, 0905.1689.

[796] P. A. Connors, R. F. Stark and T. Piran, Astrophys. J. 235, 224 (1980).

[797] A. Broderick and A. Loeb, 0812.0366. 